\title{
EACH DAY IS DIFFERENT: \\ Prison Officers and Their Work
}

By

\section{Andrea Grey NAPIER}

\author{
A thesis \\ Submitted to the Victoria University of Wellington \\ In fulfilment of the \\ Requirements for the degree of \\ Masters \\ In Criminology
}

Victoria University of Wellington

2012 
Each Day is Different: Prison Officers and Their Work 


\title{
ABSTRACT
}

\section{EACH DAY IS Different: Prison OfFICERS AND THEIR WORK}

\author{
Andrea Napier \\ Victoria University \\ Wellington, New Zealand
}

In 1991 the New Zealand prison service underwent the most significant organisational changes. Instead of homogeneity and unity within the service, with rewards being given for length of time in the job, the emphasis was now on efficiency and competition for what few promotional rewards there were to be in the new career structure. At the same time, there was to be a bigger role for women and ethnic minority officers in the prison service. This thesis examines the way in which these superimposed changes affected the working routines and the day-to-day experiences of prison officers in their aftermath. It argues that, rather than leading to a new dynamic prison service envisaged by the reformers, the prison service instead became more divided and fractious, neither management nor the prison officer body being able to keep control of some of its members, while other officers became merely perfunctory in their work and others developed strategies that undermined the 'each day is different' philosophy now projected by the prison authorities. These resistances to, and subversion of the changes were because prison officer culture, seen by the prison authorities as a barrier to reform, was not destroyed by restructuring but underwent a metamorphosis. It was reformulated and reconfigured to take account of the structural and demographic changes. This then meant that a new prison officer culture emerged, modelled around difference and uncertainty rather than cohesion, antagonism rather than unity. It could lead to deviance and corruption (which the old style prison officer culture had largely prevented) rather than dynamism and efficiency. To undertake the research, the method involved use of questionnaires covering the prison officer body of one typical New Zealand prison, in-depth interviews with 39 prison officers and nine months observation period of the everyday life of the prison officers at this institution. This aspect of the research was strongly 
influenced by Erving Goffman's dramaturgical perspective. The thesis sets out the theoretical parameters of the research as these relate to prison officer culture, and also provides an account of the methodology and the historical and contemporaneous background to the restructuring. It then provides an empirical analysis that demonstrates the effects of these changes on the everyday work of prison officers. These relate to the way in which the acculturation of the new recruits to the service became problematic because of the confusion brought about by the structural changes and the lack of experienced officers to mentor them. It then demonstrates how 'handling your lag' began to be experienced differently across the officer body, according to their length of service, ethnicity and gender. The old style unity and identity of the prison service was breaking down. This fragmentation was then exemplified by the shift to unit management. This was where power in the prison was now concentrated but, with the prison officer body too weak to regulate its use and with management increasingly distant from day-to-day prison life, this led to unpredictability and corruption. Finally, the thesis shows how many officers tried to adapt to the idea that 'each day in prison was going to be different', as promoted by the prison authorities, by trying to ensure at the same time that each day remained the same: excitement and dynamism could be tolerated only in so far as prison work remained at the same time extraordinarily mundane and routine. 


\section{ACKNOWLEDGEMENTS}

As anyone that has written a thesis can attest, the list of those that have contributed can become overwhelming, therefore I apologise in advance for any omission.

I wish to thank my primary supervisor, Prof John Pratt (Institute of Criminology, Victoria University of Wellington), and secondary supervisor, Dr Charles Sedgwick (Sociology Department, Victoria University of Wellington).

I would like to formally acknowledge Victoria University of Wellington for accepting my enrolment and access to a wide variety of resources and services, without which this project would not have been possible.

Formal appreciation must go to the Department of Corrections, John Meeuwsen (Assistant General Manager Public Prisons), Paul Monk (Regional Manager), Dave East (Regional Manager) and Dorreen Mackenzie (General Manager) for allowing me access to the pilot and target prisons and facilitating my transition into the various units. I sincerely appreciated the ease of passage, freedom of movement within your institutions and the non-directive stance you all maintained. Such openness and distance from management enabled this project to be a success. Thanks to Kim Workman for permitting me to interview him regarding gaps in the historical material.

A project of this length of time necessarily had a variety of assistance from an even more varied section of people. In the first instance, I would like to thank my mother, Esmé Ridgeway, and sister, Dr Gwynifer Napier, for their unconditional emotional and financial support. Appreciation must also be extended to my partner, Dr Simon Nelson and all my friends for their ongoing support. 


\section{TABLE OF CONTENTS}

Abstract

Acknowledgements

Table of Contents

List of Tables

List of Figures

\section{Chapter One: Introduction}

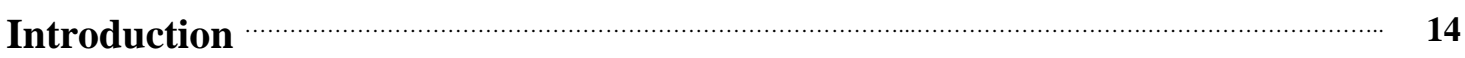

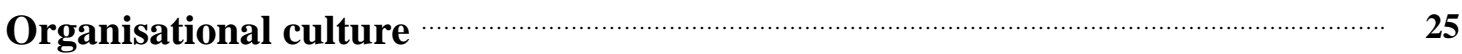

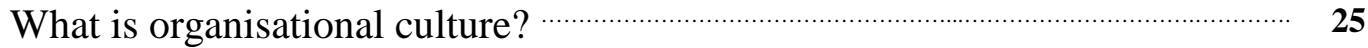

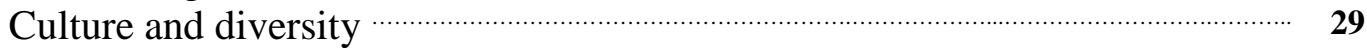

Culture and dirty work ……………....................................................................................... 32

Organisational change meets organisational culture …………………………………..... 33

Reconstructing our understanding of prison officers and their work …………..... 36

(i) A preference for inmates as subjects …………………………………………………..... $\quad 37$

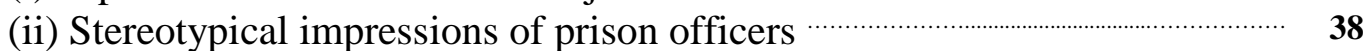

(iii) A preference for maximum security research sites ………………………........ 42

The influence of Erving Goffman ………………........................................................... 43

(i) The presentation of self in everyday life (1959) ……………………………….... 4

(ii) Asylums (1961) ……………………………….................................................... 46

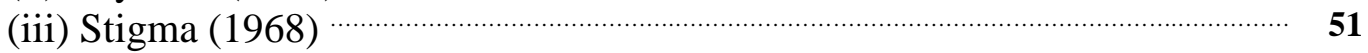

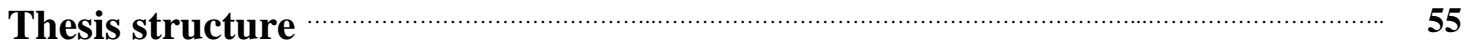

\section{Chapter Two: Doing Prison Research}

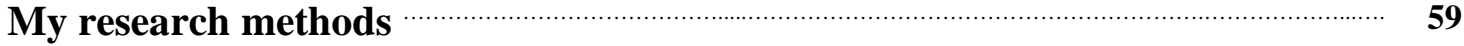

Overview of the research process

The questionnaire

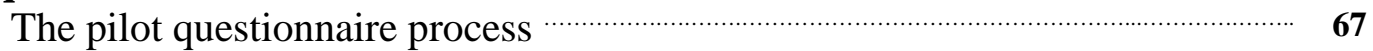

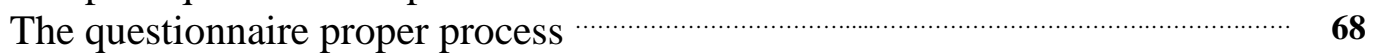

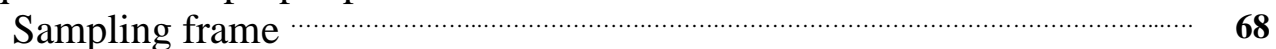

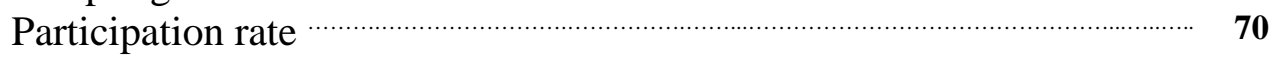

The observation phase

Obtaining informed participation ……………………........................................................... $\quad 77$

The observation process …………………………………..................................................... $\quad 79$

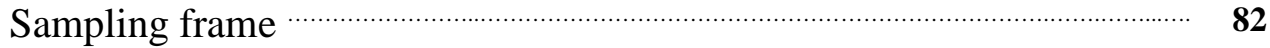

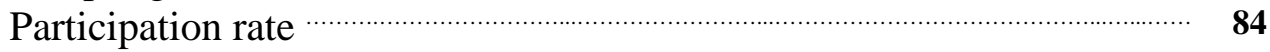




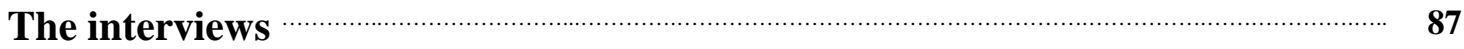

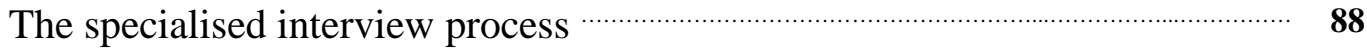

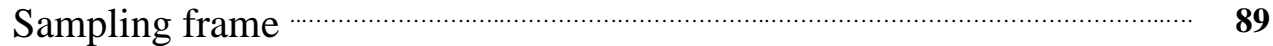

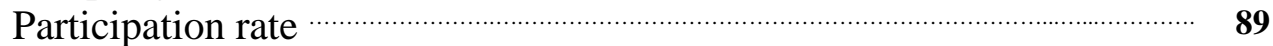

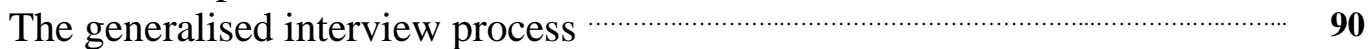

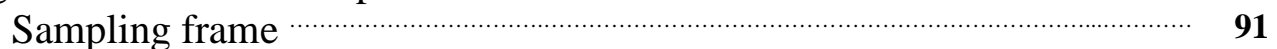

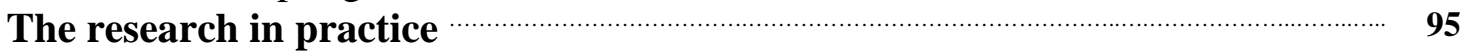

Alternative approach to ethics ……….................................................................... $\quad 95$

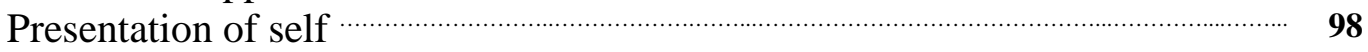

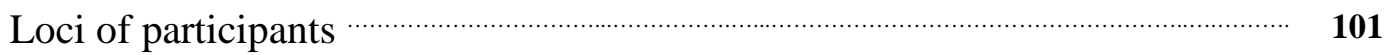

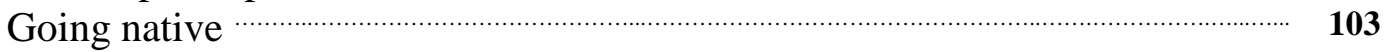

\section{Chapter Three: From Du Cane to Economic Restructuring: The} Historical Development of the New Zealand Prison Service

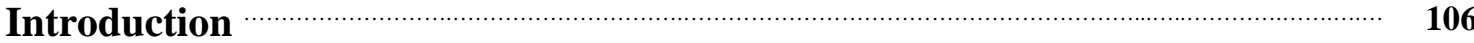

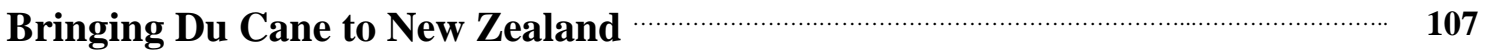

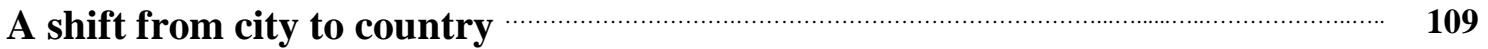

Back to security and surveillance after rehabilitation …………………………………... $\quad 113$

A new way for the prison service: 1980s restructuring ……....................................... $\quad 117$

(i) Regionalisation and unit management ……….............................................................. $\quad \mathbf{1 2 0}$

(ii) Correctionalism, case management and managerialism ……………………….. 12

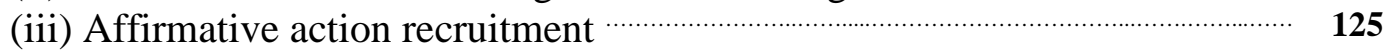

Fighting the changes …………........................................................................................... 127

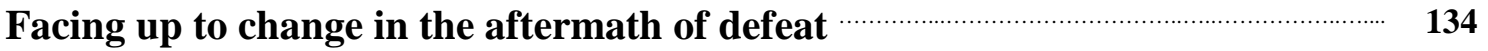

\section{Chapter Four: The Setting for this Research and the People Involved}

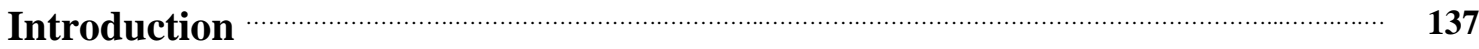

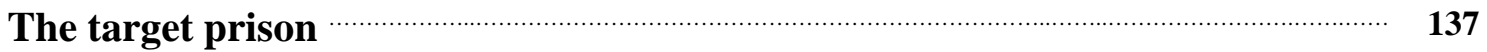

The prison officers and their backgrounds ……........................................................ 143

Similarities between prison officers at the target prison and the findings of

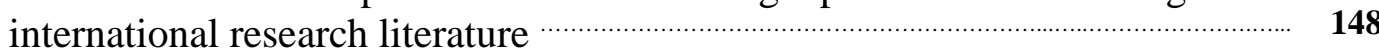

Gender ….......................................................................................................... 148

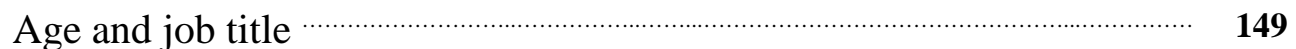

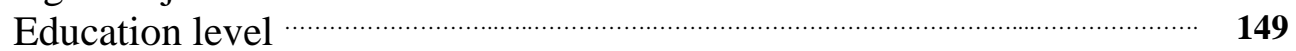

Military experience ……………............................................................................... 151

Differences between prison officers at the target prison and the findings of

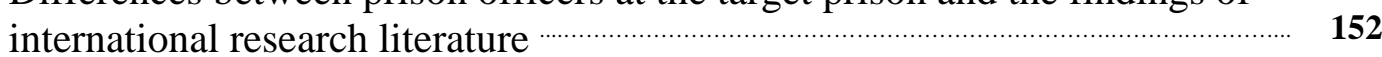

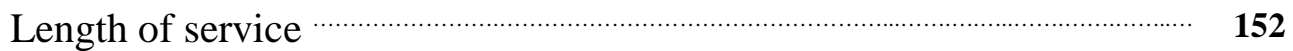

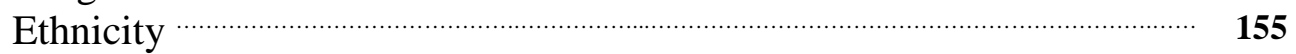

Enforced cohesion or reinforced differences and atomisation ……........................... $\quad \mathbf{1 5 8}$

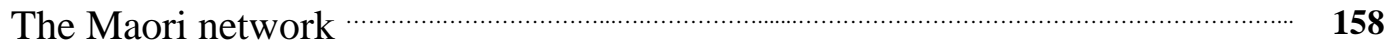

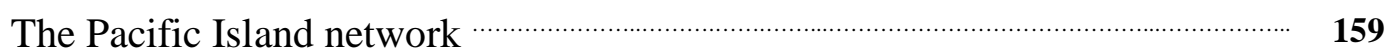

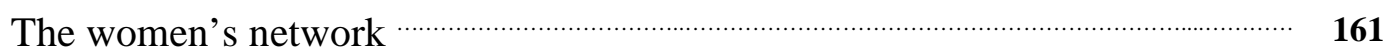

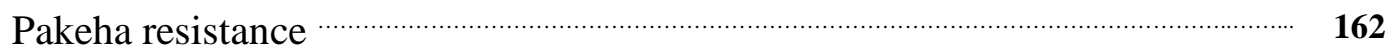

The prison officer group within the jails …………………………………………………. $\quad 163$ 


\section{Chapter Five: Learning the Scripts}

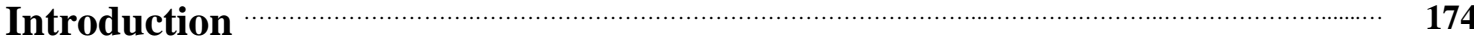

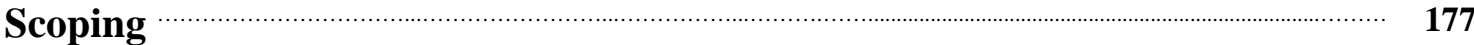

Prison college and PPOC: Learning the formal rules …………………………………….... $\quad \mathbf{1 8 5}$

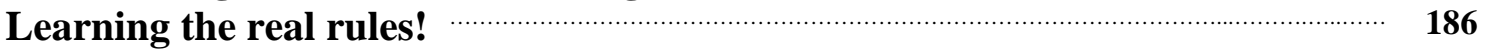

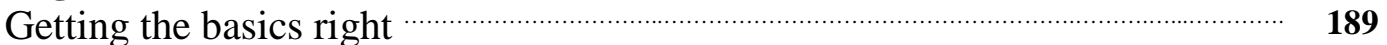

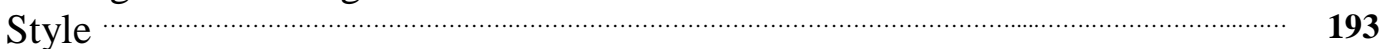

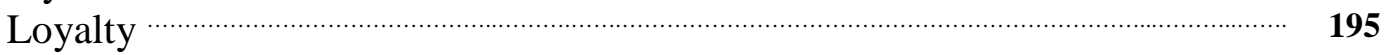

Testing and consequences of 'incorrect' behaviour ……………………........................ 199

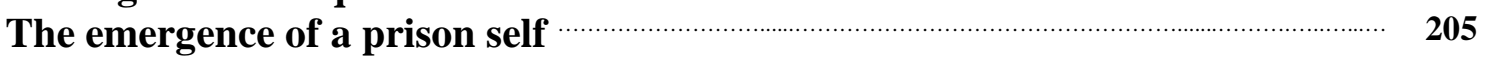

\section{Chapter Six: Handling Your Lag}

Introduction

Relationships between officers and management ……………………………................... 208

Relationships between officers and inmates …………………………….............................. $\quad 214$

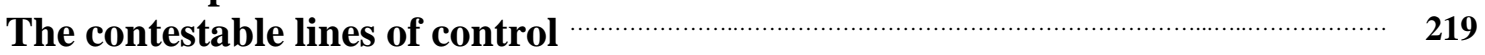

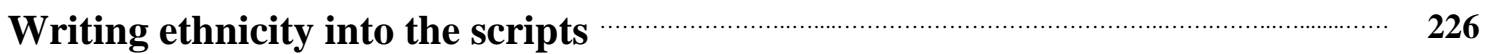

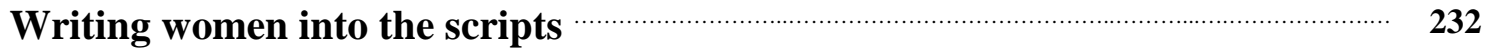

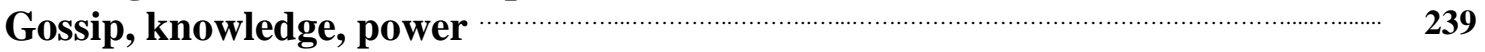

\section{Chapter Seven: Unit Divisions}

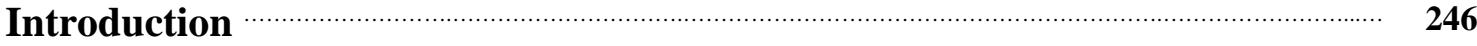

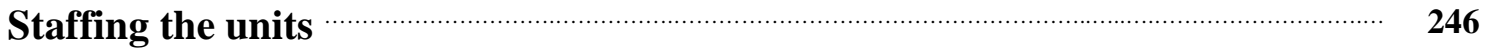

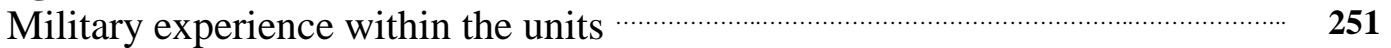

Additional jail experience within the units ………………………………………………... 251

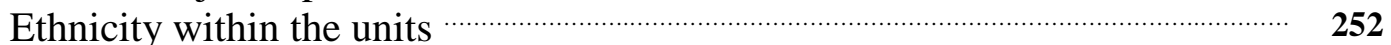

Age, length of service and job title within the units ………………………................. 255

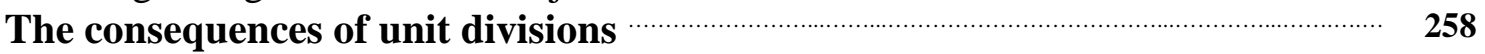

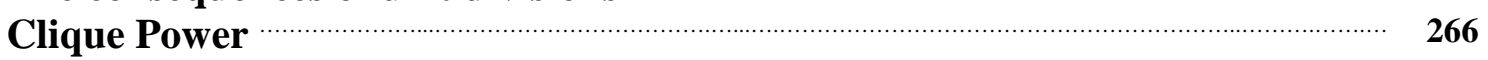

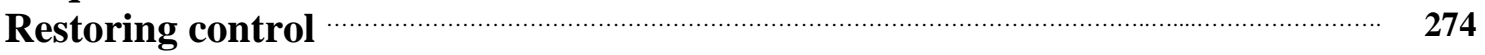

\section{Chapter Eight: Each Day Is Different?}

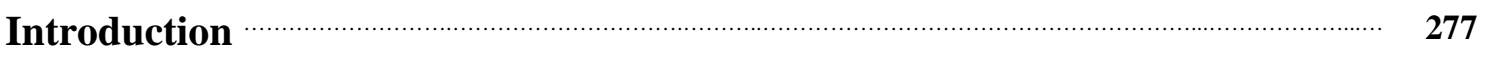

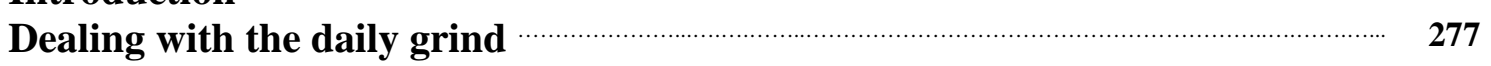

Making each day fun …………........................................................................................ $\quad 284$

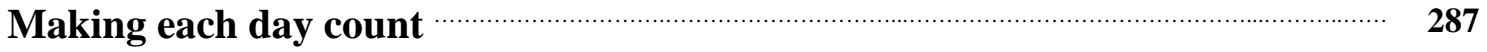

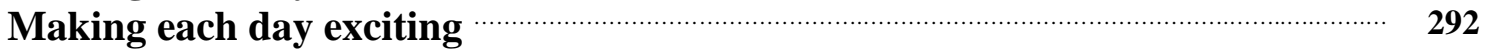

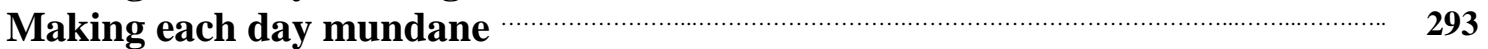

Each day is different?

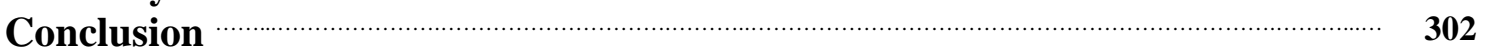

\section{Appendices}

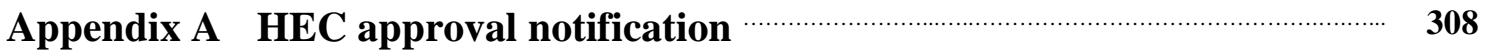




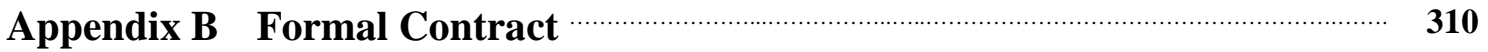

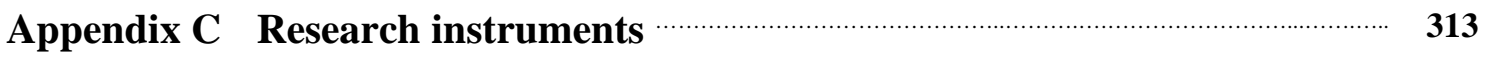

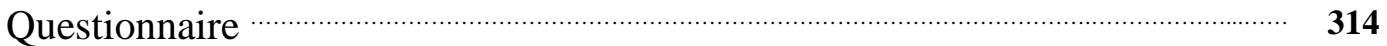

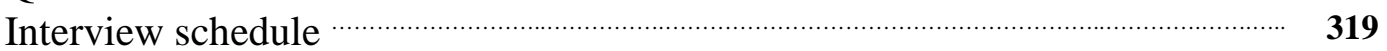

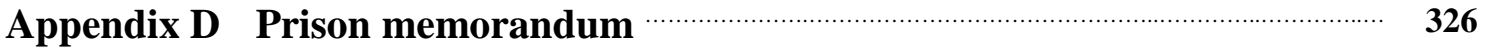

Appendix E Schematic images of the prison designs ………………….................... 328

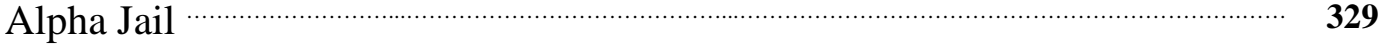

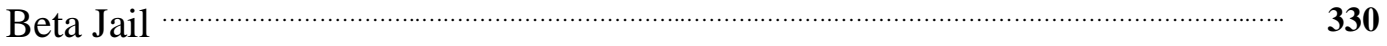

Early 1990s facility compared to 1997 facility …………........................................... 331

Complete facility at the end of the research period …………………………………... 332

Appendix F Maps of New Zealand showing prison regions and locations …… 333

Approximate prison regions …………………………………………………………........ 334

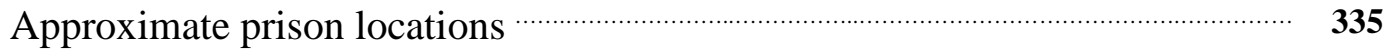

Appendix G General population growth compared to the prison

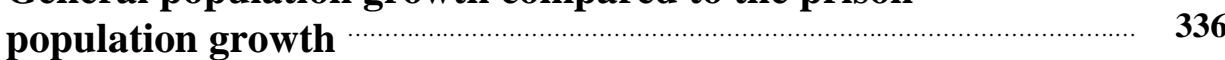

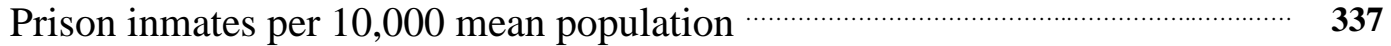

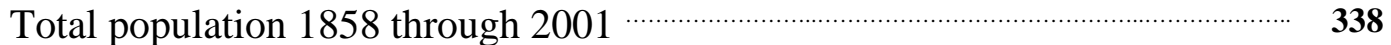

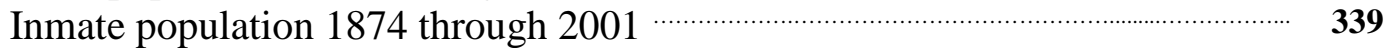

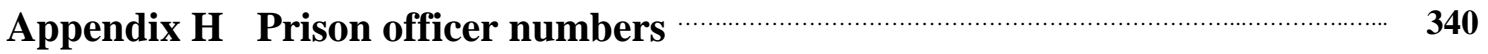

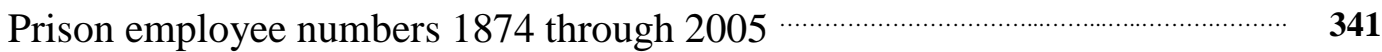

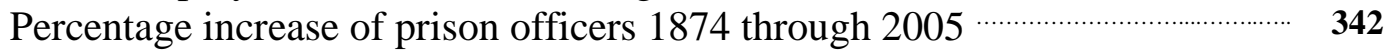

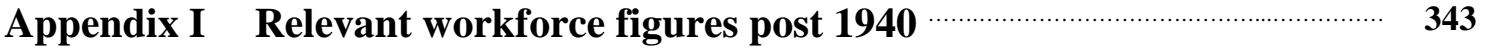

Appendix J Regional Managers' backgrounds ………………………….................. 345

Appendix K Additional notes for career structure figures …………………………... 347

Appendix L Proportion of male and female staff across institutions ……………. 350

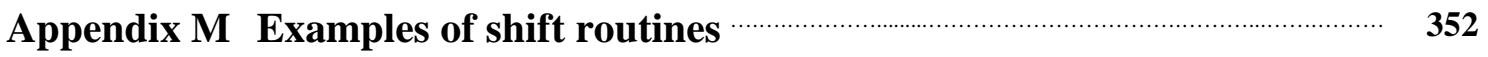

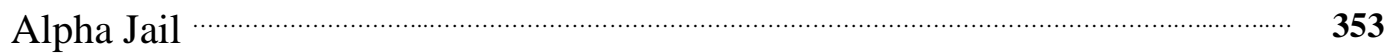

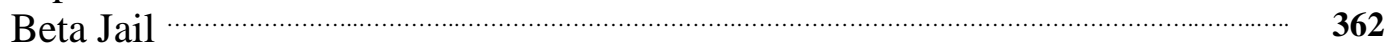

Appendix N Target prisons' cliques' and groups' demography …………………... 366

Appendix O Supplementary demographic tables for motivations for 368

Appendix P Previous occupations of prison officers at the target institution 


\section{LIST OF TABLES}

Table 2.1 Target Institution's Division with Assigned Unit Names for Easy Reference During Discussion Sections

Table 2.2 Demographic Details for the Alpha Jail Prison Officer Group Demonstrating Questionnaire Return Rate and Representativeness of Returnee Population Compared with the Total Population

Table 2.3 Demographic Details for the Beta Jail Prison Officer Group Demonstrating Questionnaire Return Rate and Representativeness of Returnee Population Compared with the Total Population

Table 2.4 Demographic Details for the Complete Target Institution Prison Officer Group Demonstrating Questionnaire Return Rate and Representativeness of Returnee Population Compared with the Total Population

Table 2.5 Summary of Breakdown of Shifts by Jail and Type

Table 2.6 Demographic Details for the Alpha Jail Prison Officer Group Demonstrating Representativeness of the Observed Population Compared with the Total Population

Table 2.7 Demographic Details for the Beta Jail Prison Officer Group Demonstrating Representativeness of the Observed Population Compared with the Total Population

Table 2.8 Demographic Details for the Complete Target Institution Prison Officer Group Demonstrating Representativeness of the Observed Population Compared with the Total Population

Table 2.9 Demographic Details of the Specialised Interviewees

Table 2.10 Clustered Demographic Details for the Male, Female and Combined Gender Alpha Jail Prison Officer Potential and Actual Interviewee Population Sets

Table 2.11 Clustered Demographic Details for the Male, Female and Combined Gender Beta Jail Prison Officer Potential and Actual Interviewee Population Sets

Table 2.12 Clustered Demographic Details for the Male, Female and Combined Gender Total Jail Prison Officer Potential and Actual Interviewee Population Sets

Table 4.1 General Job Designations of the Total Staff Group as at $11^{\text {th }}$ August 1997 ‥

Table 4.2 Demographic Details for the Total Prisons' Prison Officer Group Population as at the Start (mid 1997) and End (late 1998) of the Research Period 
Table 4.3 Total Prison: Demonstrating the Proportion of the Total Prison Within Each Demographic Category

Table 4.4 Demographic Details for Alpha Jail and Beta Jail Prison Officer Groups' Population as at the START (mid 1997) and END (late 1998) of the Research Period

Table 4.5 Jail Type: Demonstrating the Proportion of Each Demographic Category Within Each Jail Type

Table 7.1 Unit Location: Demonstrating the Proportion of Each Demographic Category Within Each Unit Location

Table 8.1 Motivations for Becoming a Prison Officer From Officers at the Target Institution

Table 8.2 Features Listed by Prison Officers at the Target Institution as the Best Features of the Job

Table 8.3 Features Listed by Prison Officers at the Target Institution as the Worst Features of the Job

Table G.1 Total Population Number for Censuses 1858 Through 2001 Demonstrating the Population Growth of New Zealand

Table G.2 Inmate Population Figures Demonstrating Inmate Population Growth From 1874 Through 2001

Table H.1 Census Data for Demonstrating Numbers of Persons Identifying as Being Employed in Penal Establishments 1874 Through 2005

Table H.2 Data Demonstrating Percentage Increase of the Total National Prison Officer Group Between Census Dates and Total National Officer to Inmate Ratio for Each Census Date 1874 Through 2005

Table L.1 Percentage of Male Versus Female Prison Officer Staff Across Institutions

Table N.1 Demographic Structure of the New Zealand Prison Officer Cliques and Groups From the Target Institution

Table O.1 Motivations for Becoming a Prison Officer by Jail Type at the Target Institution

Table O.2 Motivations for Becoming a Prison Officer by Gender at the Target Institution

Table 0.3 Motivations for Becoming a Prison Officer by Ethnicity at the Target Institution

Table O.4 Motivations for Becoming a Prison Officer by Service Length at the Target Institution

Table O.5 Motivations for Becoming a Prison Officer by Education level of Officers at the Target Institution 
Table 0.6 Motivations for Becoming a Prison Officers by Job Title of Officers at the Target Institution

Table 0.7 Motivations for Becoming a Prison Officer by Military Experience of Officers at the Target Institution

Table O.8 Motivations for Becoming a Prison Officer by Additional Jail Experience of Officers at the Target Institution .

Table P.1 Previous Occupations of Prison Officers at the Target Institution 380 


\section{LIST OF FIGURES}

Figure 1.1 Diagram of the prison service rank career structure pre-1990s

demonstrating potential career path for prison officers

Figure 1.2 Diagram of prison service managerialist career structure post-1990 demonstrating potential career path for prison officers

Figure 1.3 1970s English prison officer recruitment advertisement ……………………….... 21

Figure 1.4 1990s New Zealand prison officer recruitment advertisement …........................ 22

Figure 1.5 2000s New Zealand prison officer recruitment advertisement ………................. 24

Figure 2.1 Example of extract from field journal

Figure 7.1 Diagrammatic representation of a given prison officer's group allegiance prior to regionalisation and unit management

Figure 7.2 Diagrammatic representation of a given prison officer's group allegiance after regionalisation and unit management

Figure A.1 Copy of the Victoria University of Wellington Human Ethics Committee approval notification

Figure D.1 Representation of the prison memorandum

Figure E.1 Schematic image of Alpha Jail

Figure E.2 Schematic image of a Beta Jail unit

Figure E.3 Schematic image showing the early 1990s target facility site compared to the 1997 target facility site

Figure E.4 Schematic image of the complete target facility at the end of the research period

Figure F.1 Map of New Zealand showing the approximate prison regions

Figure F.2 Map of New Zealand showing the approximate prison locations

Figure G.1 Graphical image demonstrating the trend of inmate population per 10,000 mean general population 1880 through 2000

Figure I.1 New Zealand unemployment figures post-War demonstrating the 1960s as a period of change

Figure I.2 New Zealand income ratio demonstrating downward trend from the 1970s

Figure J.1 Previous career biographies of the Regional Managers as at the time of research 


\section{CHAPTER ONE: INTRODUCTION}

In 1991 the New Zealand prison service underwent the most radical changes since its mid nineteenth century origins. All that was previously known and understood by those working in the prisons was dismantled and then rearranged. The formal intentions of this massive restructuring were to carry the prison service forward in the wake of major reorganisation of the New Zealand state sector and public service in the late 1980s. To initiate organisational change at the prisons level, Mr Kim Workman was employed in 1990 by the Department of Justice as Assistant Secretary Penal Institutions. This was the first occasion when an outsider was hired for a senior position in prison administration. Workman had previously been employed as a senior civil servant in other areas of the public sector. Prior to the State Sector Act 1988, appointment from outside the public service above basic grade could not be made except under extraordinary circumstances. Workman's appointment symbolised the new direction of the prison service ${ }^{1}$. Length of service and progression through the ranks was to give way to qualifications, task performance and merit. Workman envisaged a complete reorganisation of the prison officers' job from its mundane security dominated tasks to a more challenging and demanding role incorporating unit and case management ${ }^{2}$. The quasi-military style organisation (see Figure 1.1) that had existed since the late nineteenth century development of modern prisons in New Zealand was abolished. The officers' five ranking levels were removed and 'overnight'

\footnotetext{
${ }^{1}$ The Ministerial Committee (1989) report outlining the prospective new direction for the New Zealand prison service was entitled Te Ara Hou, a Maori phrase that translates to 'The New Way'. The phraseology, however, was considered detrimental to the new goals for public prisons so the phrase $H e$ Ara Hou was adopted to represent the new strategies. The latter phrase translates to 'A New Way' allowing for divergence and adaptation, along with recognition that there may be alternative paths that may be explored in the future. He Ara Hou was the New Zealand version of 'Fresh Start' in Britain, albeit there were fundamental differences that influenced developments in each country. For example, while Fresh Start led to a centralised organisation, He Ara Hou initiated decentralisation.

${ }^{2}$ Unit Management was based on dividing prisons into 'units', usually sixty beds, which were separated physically and administratively from the overall prison facility and were given managerial autonomy (Sarr, 1995). Case Management incorporated the ideal of throughcare whilst in the prison system (Gendreau \& Simpson, 1986). Each inmate was given, formally at least, a compliment of programmes and services delivered from the point of reception into the prison until the end of parole. Under case management, officers within each accommodation unit were assigned between four and six inmates as their 'case load' whom they were responsible for. Case officers were to assess the inmate, propose a sentence plan of programmes, initiate access to programmes, advise the inmate on sentence matters, prepare parole reports and so on.
} 
all floor staff were to be formally considered either probationary officers or a mass of supposed equals. Meanwhile, the previous three possible management tiers became four definitive levels with a broader middle management base (see Figure 1.2). Ostensibly, the motivation for the flatter structure was to encourage and enhance teamwork within the new mode of unit management (Sarr, 1995). In reality, for many staff it meant a reduction of their salary, status and career prospects. In an attempt to solidify this movement away from a quasi-military organisation, identifiers of the old rank structure, such as the various stripes and stars on epaulettes, were formally removed. Palliatives that were given to the prison officers as part of the reorganisation included the introduction of prison dedicated drug dogs, control and restraint training (C\&R) and the creation of an intelligence unit. 


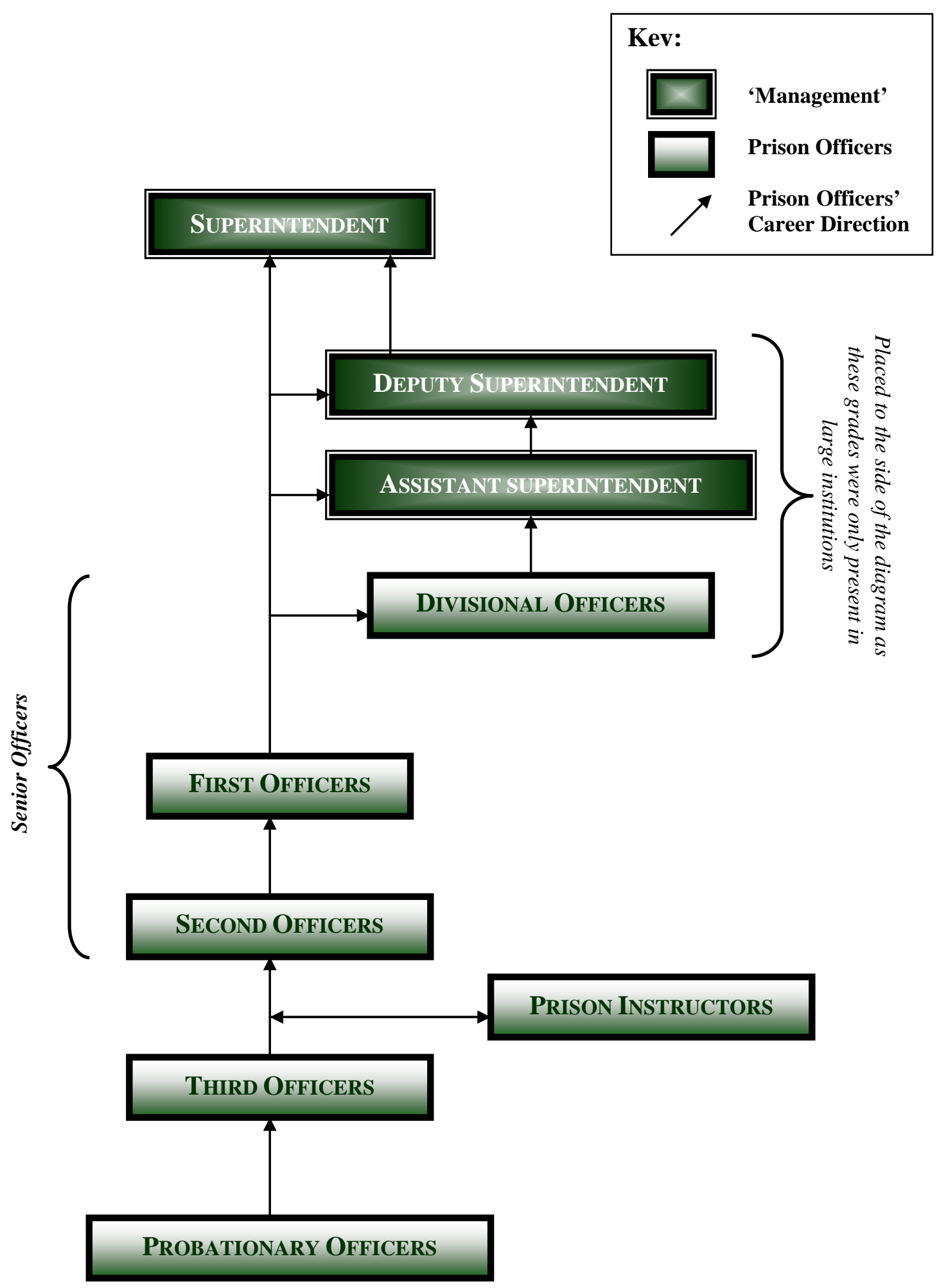

Figure 1.1. Diagram of the prison service rank career structure pre-1990s demonstrating potential career path for prison officers ${ }^{3}$.

\footnotetext{
${ }^{3}$ Information to construct this diagram was derived from various sections of the submission by the Department of Justice (1988b) to the Ministerial Committee (1989). For further details see Appendix K.
} 


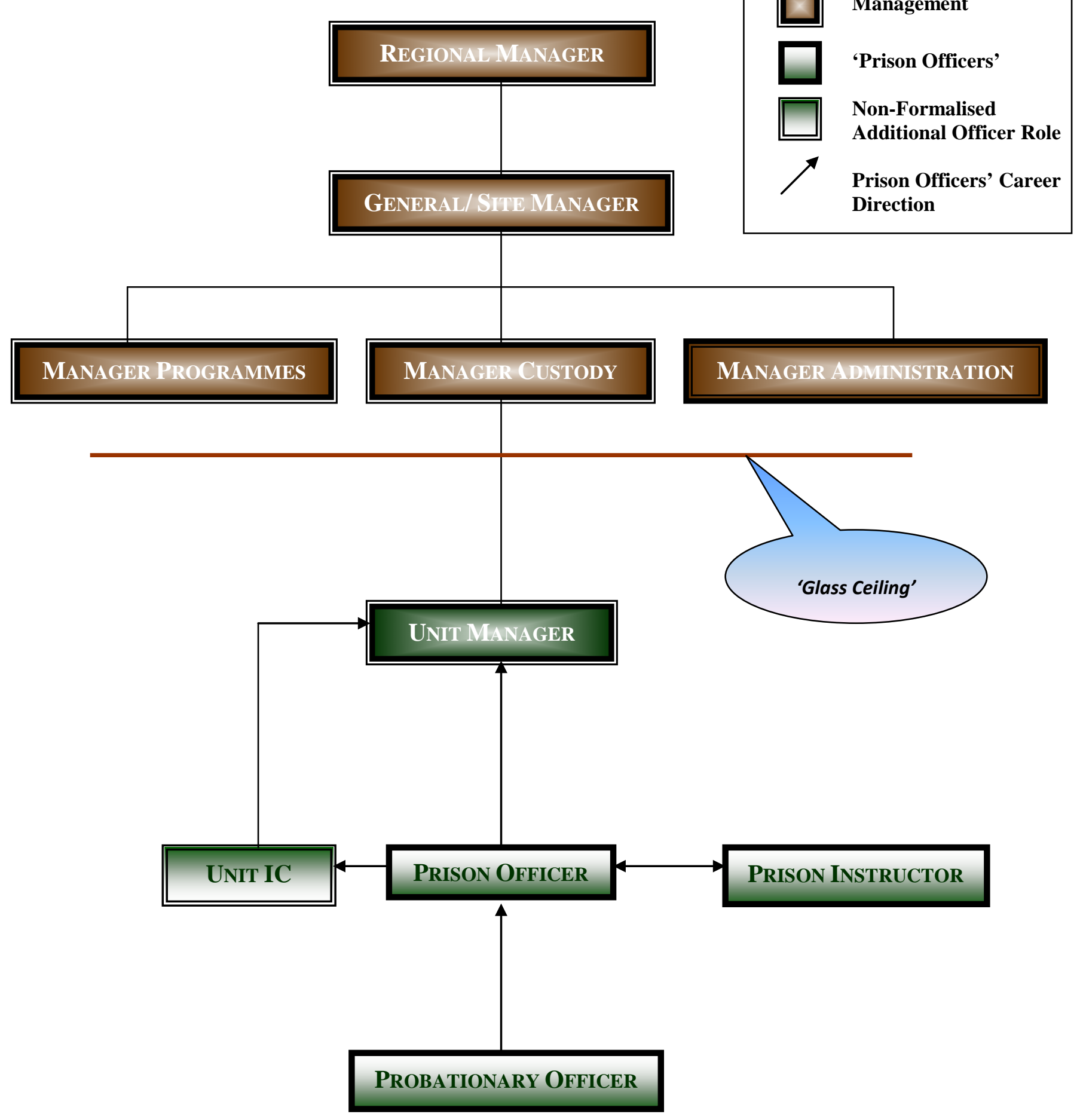

Figure 1.2. Diagram of prison service managerialist career structure post-1990 demonstrating potential career path for prison officers ${ }^{4}$.

\footnotetext{
${ }^{4}$ Information to construct this diagram was derived from the Department Justice (1988a). For further details see Appendix K.
} 
Nor did prison managers escape the restructuring process. In 1990 superintendents were moved from permanent to negotiated individual and fixed-term contracts. In this way, superintendents' positions became more tenuous, as did those of other staff members in management roles as their contracts were revised and made conditional on performance. These management positions, including those of the tier below, were initially filled with the highest ranking prison service incumbents who then had a three year fixed-term contract period to prove their transition to the new model. By 1997, when my research began, the Regional Manager and General Manager (subsequently re-renamed 'Site Manager') positions had received their fixed-term contracts and had lost their taken for granted assumptions of permanency. At the same time the Acting Unit Managers were undergoing final contract negotiations which resulted in some being returned to prison officer status and younger, more inexperienced officers taking the Unit Manager positions, making this a particularly unsettling period for those displaced. The ex-managers showed resentment and anger towards the process and result. It was similarly distressing for prison officers who had previously understood that length of service brought increased status and enhanced career prospects. This could no longer be taken for granted. Empathy was displayed by prison officers to those demoted managers who had been in the service for considerable periods of time. For prison officers, the displacement of these long serving officers reflected negatively on Upper Management and Head Office rather than on the capabilities of the given officers. Even though all Unit Manager positions were filled internally ${ }^{5}$, a significant degree of antipathy, in some cases outright hostility, was displayed by officers towards people from within the prison service whom the prison officer group felt had not progressed according to the promotion principles of their own working values - longevity of service and physical prowess. While formally these values may have been eliminated, informally they still remained firmly inscribed in prison officers’ understandings of their work.

The Department of Corrections had also intended that the new flattened career structure would motivate officers towards increased professional development (Sarr, 1995). In reality, however, the change had meant that the courses and examinations that many had taken in the past for promotion under the rank system had overnight

\footnotetext{
${ }^{5}$ The Unit Manager positions were advertised within the Departmental magazine Inside and was limited to internal applicants, though not limited to incumbents (Department of Corrections, 1997a).
} 
become meaningless and redundant. Further, given senior positions could now be filled with external personnel, few staff would be motivated towards professional development as it was unlikely to lead to career advancement. Regardless, the Department of Corrections (1997b, 1998a) established a set of nationally recognised qualifications on the NZQA ${ }^{6}$ framework ${ }^{7}$. These certificates were set at such a low level as to be achievable for virtually any officer ${ }^{8}$. This also meant that they were worthless should officers transfer to other service industries ${ }^{9}$. A new, albeit limited, progressive pay structure was aligned with this qualifications framework. The Department of Corrections claimed that this new structure was a success given that 94\% of prison officers had volunteered to be assessed by the end of 1999 (ibid, 2000a). However, as pay increments had become dependent on achieving the unit standards towards the level three certificate, this figure should not seem surprising. As an indication that prison officers were unconvinced of the utility of these qualifications, the first level four certificate was not awarded until 2001 (ibid, 2001a) and there have been few awarded since.

Another development towards increased professionalisation of the prison officer staff group was the introduction of the Code of Conduct specifically designed for Corrections (Department of Corrections, 1997e; Ministerial Committee, 1989) ${ }^{10}$. The code outlined the employer and employee expectations, principles of behaviour, specific requirements and progression of disciplinary action in the case of breaches of

\footnotetext{
${ }^{6}$ NZQA is the acronym for 'New Zealand Qualifications Authority', the official governing body of academic standards.

${ }^{7}$ This initiative was not exclusive to the New Zealand prison service. Crawley (2004) notes that the English prison service introduced a National Vocational Qualifications framework in Custodial Care in 1997. The details of the qualification and assessment are similar to those of New Zealand except that it was for new recruits and only targeted at level two. The English system was concomitantly involved in the Investors in People strategy (idem). The New Zealand system has not yet applied for such accreditation as the ISO requirements are extremely difficult for even private sector institutions to meet.

${ }^{8}$ The Certificates in Offender Management were set at levels three and four (with unit standards beginning at level two; Department of Corrections, 1997c). To put perspective on this framework, NZQA has ten levels with level five being equivalent to first year university and level eight equivalent to first year post-graduate study.

${ }^{9}$ This qualifications framework originated in Australia and the New Zealand project group aligned their development so that the New Zealand qualifications and skills were easily transportable across the Tasman, and vice versa (Department of Corrections, 1997d). However, if a prison officer wanted to move within service industries, such as to social work or probation services, level two to four qualifications would be meaningless and further qualifications at level six and beyond would be required.

${ }^{10}$ This Code replaced the Public Sector Code of Conduct, Department of Justice Code of Conduct and Corrections Operations Group Code of Practice.
} 
the code. Importantly for officers, it asserted individual responsibility for behaviour and began removing the opportunity to hide behind the anonymity of the officer group. It thus highlighted the precariousness of their employment and formally brought their personal behaviour, even when not in the prison (see the third principle below), under potential scrutiny from the prison authorities. The three over-riding principles of the code of conduct are:

First Principle: Employees should fulfil their lawful obligations to Government with professionalism and integrity

Second Principle: Employees should perform their duties honestly, faithfully and efficiently, respecting the rights of the public, colleagues and clients

Third Principle: Employees should not bring their employer into disrepute through their private activities (Department of Corrections, 1997f, p. 7; my emphasis).

Thus, while their career structure and motivation for advancement was effectively removed, new qualification frameworks and guidelines for behaviour were introduced. In these respects, it was intended that the work of a prison officer would leave behind its association with long service, mundane and banal work routines, offering nothing but unchallenging job security (exemplified in Figure 1.3, a recruitment advertisement for the English Prison Service from the 1970s), to one that was dynamic and challenging, a unique experience. Instead of each day being the same (on which the traditions of the prison service had previously been built), each day would now be different, as represented in the advertisement at Figure $1.4^{11}$. It would need to be, to meet each new challenge within the prison, and to meet the new challenges brought about by the prison officers’ reorganised career structure.

\footnotetext{
${ }^{11}$ The 1990s advertisement goes on to state "[E]pisodes like these are a part of everyday life in a prison.... Life as a Prison Officer is like nothing you have ever known before. It's extreme. It's absorbing. And it's immensely rewarding. Live it right, and you can help to reduce reoffending...”.
} 


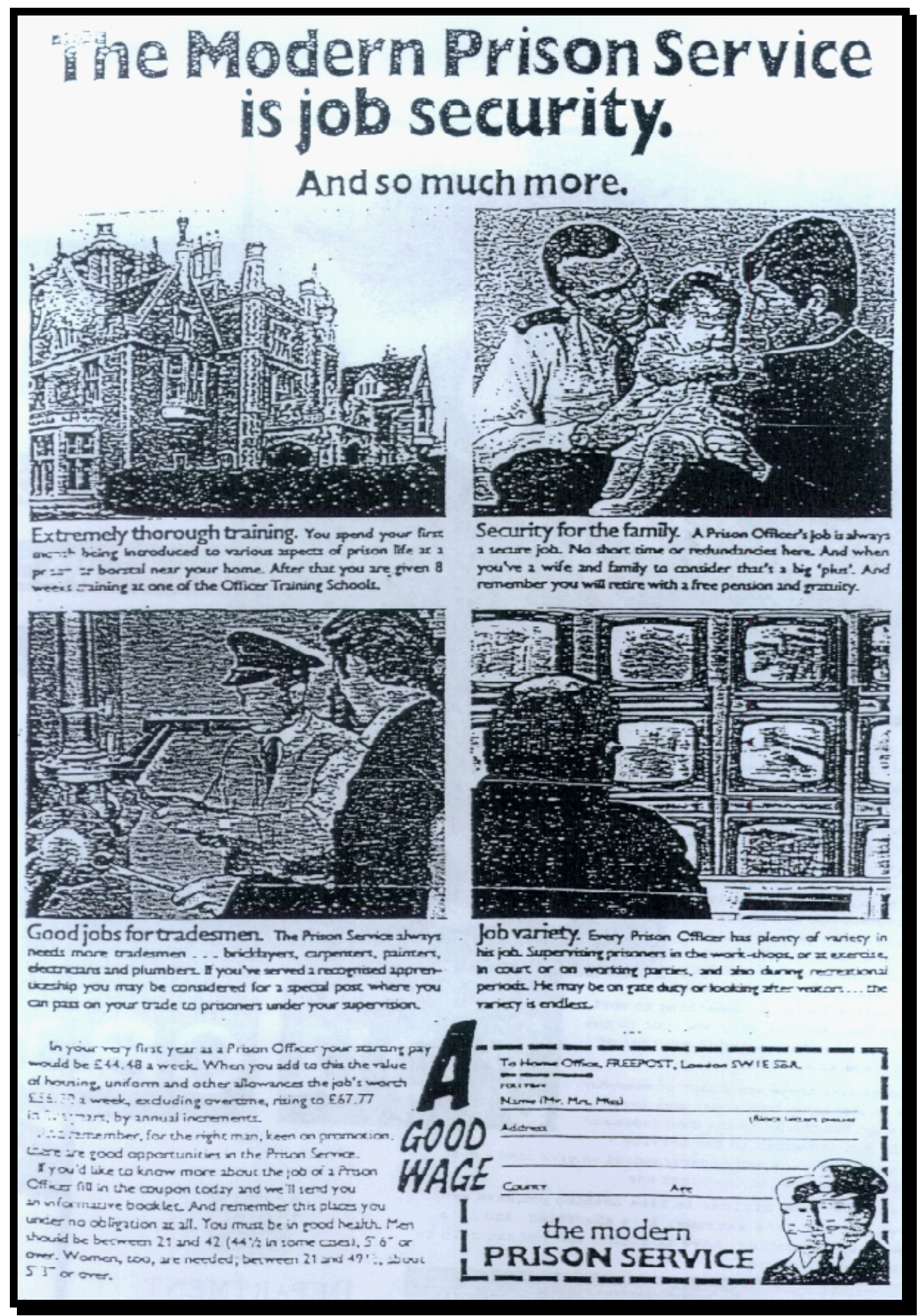

Figure 1.3. 1970s English prison officer recruitment advertisement (source unknown). 


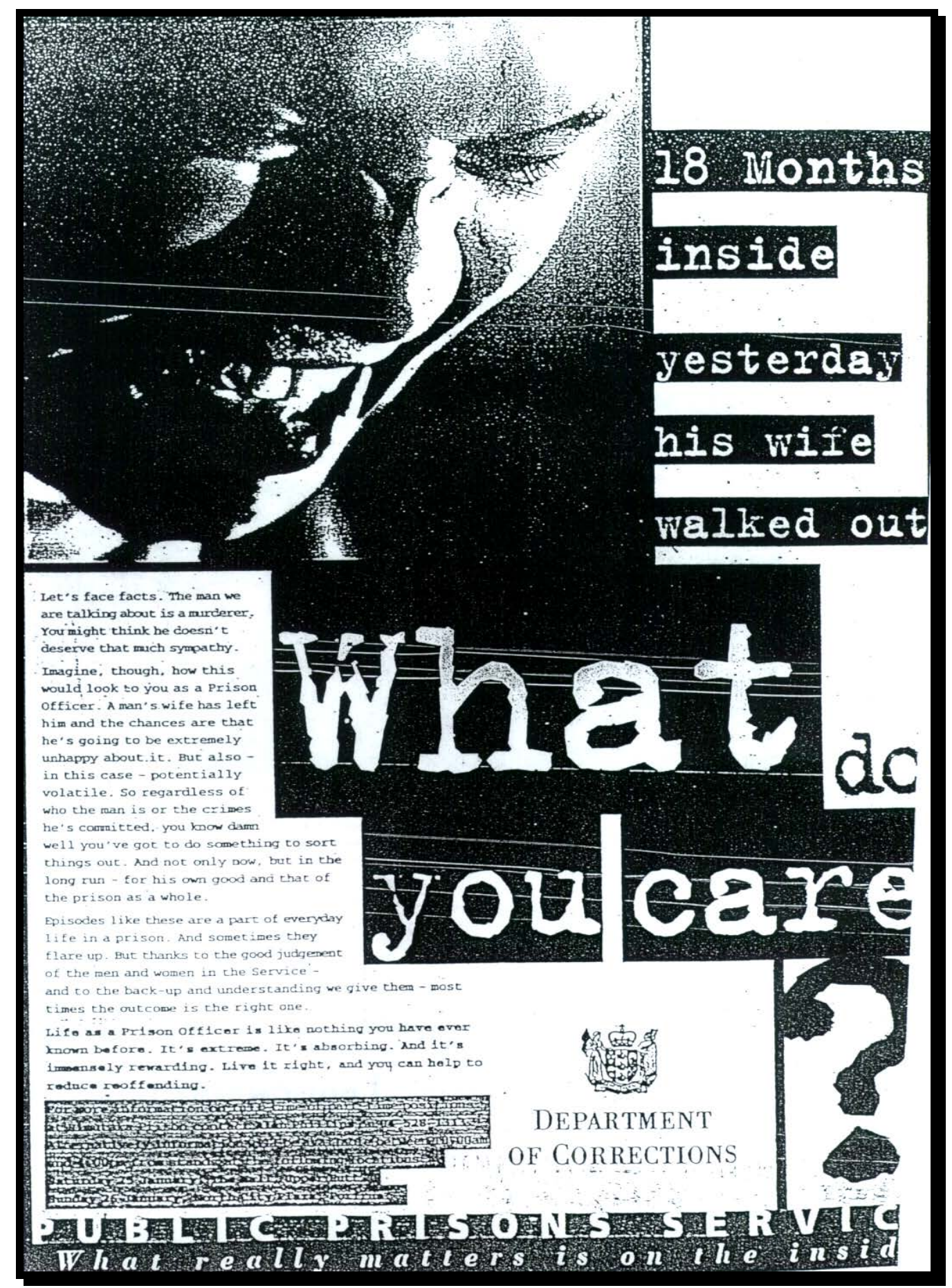

Figure 1.4. 1990s New Zealand prison officer recruitment advertisement ${ }^{12}$.

\footnotetext{
${ }^{12}$ Source: The Dominion, Job Vacancies Section, 1996.
} 
A later advertisement (see Figure 1.5) further signalled the changing nature of the prison service. In this, the white male prison officer who had traditionally dominated the prison service in New Zealand and elsewhere (see for example Thomas, 1972) has now been joined by a Maori, a Pacific Island and a woman as prison officers. It was, then, not simply that the prison service career structure was changing; the very composition of the service was changing as well.

Clearly, then, each day was indeed going to be 'different'. However, these formal expectations of what the reorganisation was meant to achieve did not seem to take into account the realities of prison work. That is, the way in which prison work, like any other occupation, is not just conducted on the basis of formal rules and, in this case, blueprints for change. In addition it is the organisational culture which tells its workers how to go about interpreting the formal rules and establishing meaning in day-to-day activities. For example, by the very nature of prison life, each day is highly routinised and perfunctory - each day is the same (see for example May, 1980). Certainty, routine, predictability and precision like timetabling have become the hallmark features of prison life since the mid nineteenth century. It was a requirement of the prison's organisational culture that each day had to be highly routinised and perfunctory for it to function efficiently (see for example Foucault, 1991); thus also ensuring that any difference-let alone each day being different—from such routines would have the potential to threaten and undermine sense-making in this micro-world. 


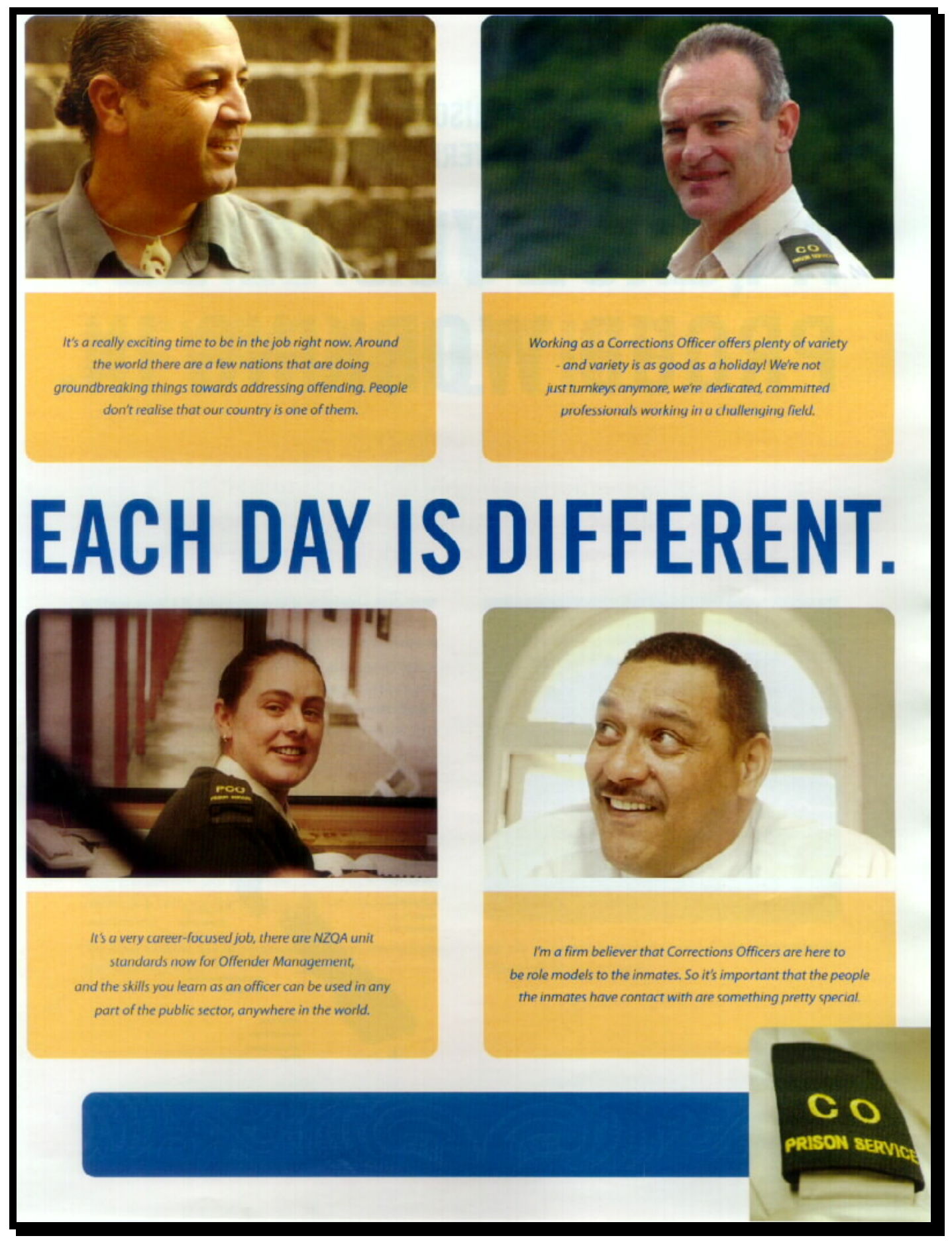

Figure 1.5. 2000s New Zealand prison officer recruitment advertisement ${ }^{13}$.

\footnotetext{
${ }^{13}$ Source: Department of Corrections (2005a).
} 
Further, such images of an exciting occupation replete with a sense of personal achievement are very much at odds with the findings of both sociological and psychological research on prisons and prison officers. These literatures document a multitude of negative features associated with the job of being a prison officer - high stress levels, low status, low intrinsic rewards, boredom and so on (see Launay \& Fielding, 1989; Lombardo, 1981; Roache, Shouksmith, Voges, \& Long, 1982; Webster, Porritt, \& Brennan, 1983; Whitehead \& Lindquist, 1986). At the same time, in much of the literature, prison officers have traditionally seemed cold, brutalising, disinterested and cynical (see Currie, 1989; Fitzgerald \& Sim, 1982). Thus, the reorganisation conflicted with both the traditional necessities of prison work, such as routine and order, and also the kinds of people who were likely to be attracted to prison work by such features as security, routinisation, camaraderie and so on (see for example Jacobs \& Retsky, 1975; Lombardo, 1981). As such, the central task of this thesis is to explore the day-to-day experiences of prison officers in one New Zealand prison as they attempted to make sense of their work in the aftermath of this dramatic organisational change. This necessitates that we examine the role played by their organisation culture in the adaptations to the changes.

\section{Organisational Culture}

\section{What Is Organisational Culture?}

In a general sense, culture is the norms, values and beliefs of a group. The term implies a sense of cyclical time and reverence, where past patterns are repeated, producing the same results time and again (Tyrrell, 2000). The addition of the term organisational implies that culture is generated or exists within an organisation. Viitanen and Piirainen (2003) referred to occupational culture as the structures of involvement, shared mission and operational principles of the organisational communities within which they reside. They argue that the construction and maintenance of occupational culture occurs through everyday speech and work practices. The culture becomes constructed as part of the narratives within which the people live their lives in relation to their organisational community. These narratives 
give both shape and meaning and are the mechanism through which action is interpreted, explained and understood.

The most common definition of occupational culture is that proposed by Deal and Kennedy (1982) who colloquially referred to organisational culture as 'the way things get done around here'. In this way, culture can be a powerful force in the workplace creating a sense of cohesion or division (Hafford-Letchfield, 2006). However, culture is not just 'how things get done' but also how workers make sense of what they have to do. The definition provided by Schein (1985, p.9) incorporates this additional dimension, whereby organisational culture is defined as:

\begin{abstract}
[a] pattern of basic assumptions-invented, discovered, or developed by a group as it learns to cope with its problems of external adaptation and internal integration - that has worked well enough to be considered valid and, therefore, to be taught to new members as the correct way to perceive, think, and feel in relation to those processes.
\end{abstract}

This definition locates organisational culture in the minds-both traditionally and currently — of members. However, another dimension to organisational culture is to be found in the way it is also understood as having a symbolic importance that is manifested in behaviour, language and artefacts. Beyer, Hannah and Milton (2000, p. 325) argue that it is "both shared ideas and symbols that help to guide organizational members to survive, adapt, and achieve collectively in their uncertain and sometimes chaotic worlds" (my emphasis). Symbols are thus observable artefacts that constitute the organisation (Schein, 1990). It is as if these reveal what is tacitly known by organisation members, even if it is difficult to articulate (Gagliardi, 1996). In addition, symbols reflect basic and shared values or assumptions, influence behaviour by eliciting internalised values and norms and facilitate members' communication about organisational life (Rafaeli \& Worline, 2000). For example, for police, their uniform is a symbol of separation and authority, just as doctors wear white coats (Young, 1991). In this way, symbols embody the meanings that integrate emotion, cognition and behaviour into shared cultural codes. In this research, with the removal of the quasi-military rank structure, prison officers were required to remove rank identifiers from their uniforms. However, prison officers refused to surrender these, continued to wear their epaulettes and referred to each other by these ranks (such as 'senior') and 
stripe level (such as 'two bar'). Ironically, while the Department of Corrections was moving away from the displays of military regalia, it continued to award long service medals and good conduct clasps. In this way, it unwittingly contributed to the maintenance of these hierarchical identifiers which it was trying to remove in other respects. Nonetheless, with the official removal of time-graduated ranks, length of service was to be considered formally irrelevant with respect to officer status. Informally, however, it would remain an important symbol of prison officer hierarchy and power relations. As Goffman (1974) argued, actors construct their understandings by framing it around expectations about what came 'before' and 'after'. Such framing is continued within an organisation through the use of war stories, myth and legend. Dandridge, Mitroff and Joyce (1980) referred to such transmission as the passage of verbal symbols which persist long after any physical symbol may be removed. Therefore, even though epaulettes have been removed from the prison service, through the transmission of verbal symbols even new prison officers include reference to the old rank structure within their communication and understandings of hierarchy and power relations within the prison officer group.

How do these aspects of culture, then, affect every day interaction and understandings of work? Bandura (as cited in Baldwin \& Baldwin, 1986) argued that behaviour becomes associated with symbols which then act as cues for future action. For example, research by Rafaeli and Pratt (1993) demonstrated the effect of observing a police uniform makes people accept orders unquestioningly, even if they had never interacted with the police before. Merely the symbol of the uniform brought appropriate action from the observer. In this way, culture not only provides a means of sense making within organisational life but also guides behaviour within its context. As Whiteley (1995, p. 19) proposed:

The culture of an organization, like that of a society, is a homemade blueprint for seeing the world in a particular way.... People decide what they value greatly and they build their beliefs, attitudes and ultimately behaviours accordingly. The blueprint takes the form of behaviour rules which have become solid enough to form everyday, well-accepted patterns of behaviour that everyone knows even if they don't say so. These patterns and rules will be handed down as organizational memory or folklore. In the case of organizations the values and beliefs will provide the spectacles through which members will interpret organizational life. (my emphasis) 
Two important points are highlighted in this quote. First, organisational culture is composed of particular sets of behavioural rules. Helms Mills and Mills (2000) differentiate between formal and informal organisational rules. Formal rules are those that are associated with the pursuit of organisational goals, purposes or activities. Informal rules arise from the context of the employee associations but are not necessarily aimed at meeting organisational goals, purposes or activities. That is, in the process of making group associations, norms emerge that govern aspects of members' behaviours. Such norms may be complementary to formal rules or contradict and subvert them. Not only this, Helms Mills and Mills also argued that informal rules have the most significant influence in the day-to-day experience of an organisation and its members. Further, although such rules are the result of actors' interactions, they take on an existence above the members and become inextricably linked to the culture of the organisation. In this way, they have an existence independent of individual workers and become part of the normative framework that instructs them on 'how to get this job done' and 'how to make sense of it'.

Secondly, it is the taken for granted nature of the values and behaviours within a culture that maintains its existence (see also Herselman, 2004). As Carlopio, Andrewartha and Armstrong (2005, p. 362) asserted “[a]ctions that are congruent with the prevailing value system in an organization are deemed credible, or legitimate, by other organizational members. They are taken for granted, rather than challenged or scrutinised”. Actions or beliefs that are not congruent are deemed deviant and discouraged: they are simply incomprehensible and ignored or threatening and confronted. Similarly, as actors find it difficult to articulate the values and assumptions of their culture, it is difficult to challenge them as they are simply 'the way things get done around here’.

In these ways, culture gives the organisation a personality and provides its members with a sense of identity and commitment to something larger than self-interest. The basic functions of organisational culture provide stability through shared values, provide a control mechanism by shaping employees' attitudes and behaviour and provide cohesion through the shared sense it gives individual employees of belonging to a group. Kono and Clegg (1998) proposed that the functions of culture from the members' viewpoint are that it enables simplification and adaptation of decisions, 
affiliation, motivation, activation and a sense of purpose (see also Pedersen \& Sorensen, 1989). The function of culture for an organisation was first recognised in the Hawthorne Experiments ${ }^{14}$ (see Hawthorne Experiments, 2008). Mayo, cited in Fineman, Sims and Gabriel (2005), added to the findings of the Hawthorne Experiments by noting that organisational groups not only establish the motivation for activity, but also determined the norms by which the group worked. Norms not only instilled conformity to the group, but also controlled deviant behaviour and effected productivity. In this way, organisational culture is important for the employees, employers and the organisation.

To effectively understand and communicate within an organisation requires intimacy with the culture which transforms the individual in ways that replace any former assumptions with cultural world views. In this way, a new 'self' can be seen to emerge (see also Goffman, 1961). However, assaults on the culture, such as during imposed change, are then experienced as assaults on the self - thus even if the change is positive, it will be opposed by members if it destabilises the existing status quo (Carlone \& Larson, 2006; Hatch, 1993). As an example, Marquis and Lounsbury (2007) highlighted the significant displays of resistance by organisational members to the acquisition, mergers and centralisation of control of various banks in USA, even though the result of such business changes was increased opportunities and rewards for the majority of employees. Similarly, Sopow (2007) asserted that even if a culture is unhealthy for its participants, change is incredibly difficult as people tend to prefer "the imperfect predictable to the perfect unpredictable" (p. 21).

\section{Culture And Diversity}

Nonetheless, organisational culture is not static. Members are continually creating meaning from symbolic artefacts and responding to this meaning. This allows for

\footnotetext{
${ }^{14}$ The Hawthorne Experiments were a series of experiments conducted at the Hawthorne Works factory between 1924 and 1932. The initial purpose of the experiments was to examine the effects of lighting on worker productivity. A second set of experiments, conducted by Roethlisberger and Dickson (cited in Hawthorne Experiments, 2008), examined the effect of other changes in the working environment. Although the methodology of these experiments has been widely criticised, Mayo (cited in idem) concluded that the important variable in the various experiments was the influence of working in groups.
} 
variable appearances of the culture depending on who is on shift or within the vicinity of the action (Hatch, 1997, 2000). Such levels of change are tolerated by the culture as routinisation of this 'change’ permits continued stability. This is similar to Gagliardi's (1990) notion of incremental cultural change whereby such 'new' meanings are juxtaposed to old and therefore merely extend the reach of the basic understandings. Van den Berg and Wilderom (2004) extended this argument by proposing a limit on organisational culture to shared perceptions of organisational work practices within organisational units which they asserted may differ from other organisational units. In effect, rather than one culture being characteristic of a particular occupation, we may well find multiple cultures arising, crossing and intersecting. Kakabadse (1982) proposed that different organisational cultures within the one organisation arose when various groups experienced situations differently. It is the perception of these experiences that creates the common culture within the various subgroups. To illustrate, consider the police and their organisational culture. While there is an overarching occupational culture within the police (see for example Chan, 1997, 2003), research also indicates that there are various subcultures within it such as 'street' police officer culture (see for example Reuss-Ianni \& Ianni, 1983); detective culture (see for example Innes, 2003); women police officer culture (see for example Brown \& Heidensohn, 2000; Heidensohn, 1992; Remmington, 1981; Westmarland, 2001); ethnic police officer culture (see for example Holdaway \& O’Neill, 2006); and gay police culture (see for example Burke, 1994).

The existence of multiple subcultures is neither inherently good nor bad, depending on the fit between the subcultures and the organisation and the ultimate goals of the organisation (Gabriel, 1994; van den Berg \& Wilderom, 2004). However, the prison service is historically reflective of what Härtel (2004) referred to as a diversity closed group (see for example Thomas, 1972). Prison officers had developed as a homogenous set of workers (see chapter three). Accordingly, any trait that represented a 'difference' to that homogeneity was experienced as threatening and unacceptable. Such groups have a generic attitude to difference prior to interactions. Kono and Clegg (1998) identify gender and age as central to the development of multiple 
cultures. Arguably, ethnicity would also influence subcultural development ${ }^{15}$. There is an expectation that dissimilar members will assimilate to the existing culture without the culture accommodating any of the new members' characteristics. Although the espoused culture of the public prisons service in the post 1990s change period was supportive of diversity, as evidenced by the assertive equal employment practices, the internalised culture did not reflect this element ${ }^{16}$. To illustrate, consider the introduction of women officers to male prisons. This was typically opposed by male officers who put so much value on machismo or masculinity in carrying out their work (see Britton, 2007; Jurik, 1985a, 1988; Owen, 1988; Zimmer, 1989). Wilson (1995) noted that female employees tend to be characterised according to gendered stereotypes and evaluated less favourably than men. Arguably, these stereotypes, as basic assumptions and beliefs, are integrated within the organisational culture. This is compounded when the existing culture supports machismo and masculine stereotypes, as in the prison. In such an environment, perception of danger that can only be encountered by drawing on masculine qualities, limits the status of women and their perceived ability to fulfil this role. Similarly, as a result of the gendered interaction of the group, women resist gender stereotyping making their position a constant challenge as men continue to impose their masculinised limits on their activity and inclusion (Helms Mills \& Mills, 2000).

Lupton (2000) asserts that men working in female-dominated occupations fear stigmatisation and feminisation. The common response is for the male to engage in compensatory gendered practices so as to minimise the feminine associations and restore a sense of masculinity. In relation to the changes in New Zealand prisons, this would suggest that the introduction of the direct care role of case management, arguably a feminised role, would be met with some resistance and compensatory practices by the predominantly male staff. In her analysis of gendered roles in the caring industry of nursing and primary teaching, Simpson (2007) found men actively

\footnotetext{
${ }^{15}$ For example, in a comparison between 'black' and 'white' garbage men, Walsh (1975) found very different coping mechanisms, work attitudes and behaviour (culture), self-esteem levels and identity effects between the two groups of workers. Although Walsh did not state it explicitly, there were two distinct organisational cultures originating from ethnicity.

${ }^{16}$ According to Kono and Clegg (1998) the espoused culture is the officially sanctioned culture of the organisation which members may or may not view as authentic. The internalised culture is based on the real beliefs and enduring patterns that members operate within on a daily basis. At any one time, either the espoused or internalised culture may be the dominant culture - in the case of the prison, the internalised culture dominates (see for example Kauffman, 1985, 1988; Sykes, 1958; Thomas, 1972).
} 
chose 'masculine' specialist functions and the more authoritarian and challenging roles. It could therefore be hypothesised that the introduction of the 'caring role' in the prison service would similarly compound the gender division between staff with women officers having to fulfil the 'feminised' aspects of the job. Indeed, Britton (2007) found distinct gendered assignment of roles within male penal institutions. Farnworth (2007) cited sexual harassment, tokenism, lack of required physical characteristics, inequality of assignment, lack of acceptance into the occupational socialisation process and resentment from their male colleagues as integration difficulties of women entering the prisons as prison officers.

\section{Culture And Dirty Work}

It may well be, though, that prison officer masculinity is simply an extreme form of such values which are legitimated by society in general, namely assertiveness, machismo, virility and brotherhood. It is the extremeness that makes it interesting for investigation. It takes an extreme form because the work of prison officers itself intersects a number of occupational extremities. For example, there were striking parallels between Walsh's (1975) research on garbage men and the prison officers of this research. Such similarities between these two sets of workers include: highly sexualised focus of conversations; verbal derision of management's assumed idiocy; reluctance to tell outsiders their occupation; reframing strategies for establishing meaning and worth in their occupation; forming intragroup stratification systems as a means for identity and self-esteem; inability to find another job with equal pay given their low education levels; use of 'war stories'; passing time with 'fun' competitions such as who can complete their route the fastest (with status attributed to those in the lead positions); and arriving early for shift to bond with colleagues. In effect, both sets of occupations involve 'dirty work': work, that to the general public, involves working with 'garbage' (human or otherwise) and which carries with it stigma and disdain from most other members of society. Walsh found that garbage men mitigated the effects of their dirty work on their selves by putting a high value on job security and pay and by enhancing the status of the occupation in their own eyes by allowing their occupational culture to be particularly cohesive and bonding. For example, Walsh found that, in comparison to parks occupational workers who in some respects 
performed a similar job, the garbage men seemed to have a much stronger occupational culture, as if this then helped to cope with the stigma that was attached to their work, but not to that of the parks workers. Prison officers, too, have a stronger culture than similar occupations that do not exclusively deal with what the general public would see as the detritus of human existence, such as firemen.

Notwithstanding this, the prison is also a uniformed organisation. Employees of such uniformed occupations display greater levels of natural identification, institutionalism and parochialism compared to other organisations (Soeters, 2000). Typically, these have their own training academies and qualification structures, further denoting their uniqueness. Their work may be dangerous and employees are trained in the use of legitimate force. Another unique feature is that such organisations are 'greedy organisations' due to the level of commitment and duty required of its members. Soeters notes that the communal style of life is not limited to those that are physically confined to barracks, but is a feature of uniformed organisations generally as the organisation and work extends into the employees' personal life more than in nonuniformed organisations. Uniformed organisations tend to have an emphasis on authoritarian ideology, discipline and control and a downward flow of directives. By the nature of their work, they are also likely to be very suspicious and mistrusting of outsiders. Of all these occupations, though, prison officers are likely to be at the bottom of any such hierarchy: much less publicly visible and much more preoccupied with the 'dirty' nature of their work as opposed to the heroic potential of the other uniformed organisations. On this basis, we should thus expect to find a particularly strong culture amongst prison officers that provides additional support and bonding for them to help them get the job done, make sense of their work and provide a barrier against all the stigma associated with their work.

\section{Organisational Change Meets Organisational Culture}

As has been illustrated, organisational culture is important for its members as a means of determining 'how things get done around here' and also as a way of allowing workers to make sense of and understand their organisation and their role in it. At the 
same time, there can be multiple cultures within the one organisation. In these respects, we want to examine in this thesis how the previously diversity closed and extreme New Zealand prison officer culture accommodated the recruitment of women and ethnic male officers to its ranks, in addition to the restructuring that was being imposed on it. Kakabadse (1982) noted that a frequent mistake of change agents is to ignore the effect structural changes will have on attitudes and work behaviours and work cultures. Another was that change agents fail to recognise the importance of culture and thereby assume that culture will change in intended ways or that it will simply adapt itself to the new blueprint. Furthermore, in the process of revolutionary change within an organisation, the entire culture and meaning making process will be destabilised. Importantly, when such change is imposed organisational culture itself will change - it is not possible to simply remove culture-but it is likely to change into something unpredictable and unforeseen (Baker, 2007). If an existing culture is undermined in such a way as to irrevocably weaken it, something will replace it. If change strategies are initiated that do not account for the organisational culture's assumptions, employees will not live with the contradictions of understandings for long (Whiteley \& Whiteley, 2007). Indeed, even the most well planned change strategy is likely to fail if the role of culture is not taken into account (see for example Baker, 2007; Galpin, 1996). Resistance to change is normal (see for example Burns \& Stalker, 1996). As Machiavelli (cited in Dwan, 2004, p. 36) warned:

\footnotetext{
There is nothing more difficult to carry out, nor more doubtful of success, nor more dangerous to handle, than to initiate a new order of things. For the reformer has enemies in all who profit by the old order, and only luke warm defenders in all those who would profit by the new order. This lukewarmness arises partly from fear of their adversaries... and partly from the incredulity of mankind, who do not truly believe in anything new until they have had actual experience of it.
}

This resistance is linked to nostalgia. For Gabriel (1994), organisational nostalgia arises out of the present conditions as much as out of the past itself. The past is recalled in a highly selective mythologised ideal, infused with symbolism and meaning. It tends to be embellished and juxtaposes the present as somehow deficient in comparison with the past. This re-presentation of the past necessarily affects the experience and behaviour in the present. Further, employees that experienced the 'old' 
are bound by that shared heritage and tend to exclude those employed after the change, leading to division and confrontation.

How does this relate to a public sector organisation such as the New Zealand prison service? Public sector organisations, with longstanding histories, institutional memories and deeply embedded culture are likely to be particularly difficult to change (Baines, 2006; Baker, 2007). The 1990s developments provide a particularly interesting scenario given that the dominant strategy for coping with the philosophical changes that emerged in the New Zealand public sector following the 1980s restructuring was to adopt private sector practices (Parry \& Proctor-Thomson, 2003). This, Parry and Proctor-Thomson argued, did not take into consideration the unique characteristics of the public sector which required maintaining a focus on not-forprofit activities and accountability to a diverse range of stakeholders. These characteristics require stability and restraint of innovative propensities rather than the flexibility and innovation invoked by the private sector model (see for example Burns \& Stalker, 1996).

The conflict between private sector working practices imposed on public sector organisations was particularly evident in the prison service. For example, Harrison and Stokes (1992) suggested that all organisations, private and public sector, consist of a combination of four distinct cultural orientations: power, role, achievement and support. Prisons contain an historical base of the power-oriented culture whereby there has been clear control and direction from a leader (Superintendent or Chief) with the motivation of employees resting in rewards (such as camaraderie, job security and a malleable remuneration system) and a desire to be accepted by higher ranks. A strong current of the support-oriented culture also permeates this power culture whereby commitment to fellow employees and the prison service are highly valued. In addition, elements of the role-oriented culture have also been a traditional feature of prisons due to the quasi-military structure of the prison service. The feature that had been missing from the prisons had been the achievement-orientation culture, yet this is the base that is required in the post 1990s restructuring of the prison service. In this way, the prison service did not have one of the crucial attributes that would be needed to implement the private sector model that had been imposed on it. Although the Workman initiatives attempted to import the achievement-orientation culture with the 
introduction of case and unit management, these values were imposed on the existing culture rather than evolving from within it.

Furthermore, as Bass and Avolio (1993) have argued, the ideal culture type for organisational change was a transformational culture. Transformational cultures support innovation, open communication and view challenges as opportunities rather than threats. Such cultures maintain the stability required to enhance employee's motivation and agency. However, the public sector tends to have transactional rather than transformational cultures. Transactional cultures contain individuals lacking commitment to the organisation's mission except to the extent that rewards are provided. Parry and Proctor-Thomson (2003) thus found that the New Zealand public sector lacked transformational organisational cultures - hence resistance to change was likely to be the result. As this began to occur, it was enhanced by the development of 'backward glancing sensibilities' which increased employees' attachment to past practices (Loader \& Mulcahy, 2003), the more so when the promises of change agents do not materialise. For example, as Loader and Mulcahy illustrate in relation to policing, and as I will go on to illustrate in this thesis in relation to prison officers, organisational change was 'sold' to these two groups of workers in the 1990s on the basis that bureaucratic routines and 'paperwork' - one of their biggest bugbears—-would be reduced. In reality, the opposite occurred.

\section{Reconstructing Our Understanding Of Prison Officers And Their Work}

The purpose of this thesis is to investigate the effect of these changes on the everyday working life and understandings of prison officers. By the late 1990s it was clear to prison officers that the substantive changes of the early 1990s were now a reality of prison work - and no amount of resistance would alter this. To function on a day-today basis and make sense of their work they needed to accommodate these new features of their job. To examine how they did this, this thesis employed an ethnographic research methodology to critically analyse prison officers' adaption to 
the 'new' prison service within a mainstream or 'typical' prison. As such, this research diverges from much of the literature on prisons in three major respects:

\section{(i) A Preference For Inmates As Subjects}

Prison research has been dominated by the emphasis given to prison inmates, 'the subordinates' of prison life. Until recently at least, there has usually been only a cursory discussion of other participants within the prison system (see for example Cavadino \& Dignan, 1993; Fitzgerald \& Sim, 1982; Matthews, 1999; Sykes, 1958).

This focus can be traced back to the work of the founding father of the sociology of imprisonment, Donald Clemmer (1940), and subsequent influential authors such as Gresham Sykes (1958). The focus on inmates and their culture continued during the prison research boom of the 1960s with such classic studies as Cressey (1959), Cloward (1960), Sykes and Messinger (1960), Wheeler (1961), McCleary (1960), Irwin and Cressey (1962), Garabedian (1963) and Giallombardo (1966) all demonstrating the wider social science preference for the 'underdog'. Such studies provided the seminal foundations of prison culture research and, without exception, placed inmates in the centre of the analysis. It simply became accepted within the genre that inmates were the subordinates and underdogs of the prison community and hence the most worthy subjects for investigation.

As the inmate group demonstrated their adaptation to this confined world more visibly than prison staff, this emphasis on them might seem to be rightly placed. Coinciding with this preference for the 'underdog' there has been a tendency to romanticise such subjects. In a good deal of the sociology of prison literature, the inmate lost the presentation of self as an individual who had offended against society's laws sufficient to be excluded from society for a period of time as punishment. In the romanticised view, this individual was presented as the victim of a wider set of social circumstances past and present, rejected from society, and who now had to recreate meaning and purpose while enduring psychological and physical subjugation (see for example Cohen \& Taylor, 1972; Goffman 1961; Johnson \& Toch, 1982; Parisi, 1982; Piacentini, 2004; Zamble \& Porporino, 1988). 
However, maintaining inmates as the research focus and romanticising their situation is problematic. While they represent the majority group within the prison, they are only one side of the social organisation and dynamics of the prison. On the other side of this division are prison officers who are also fundamental to prison life and the functioning of the institution. Arguably, an understanding of social dynamics, both within and between social groups, are an essential element in the analysis of any behaviours that take place within that context (see for example Berger \& Berger, 1978; Giddens, 1991; Robertson, 1989; Schaefer, 1989).

\section{(ii) Stereotypical Impressions Of Prison Officers}

While the research literature on prison inmates began to accumulate, what there was on prison officers tended to be in the form of autobiographical accounts and Prison Officers’ Association publications (see Jones \& Cornes, 1977). While this work may be valuable in its own right, it is unlikely to be of social scientific validity or permit generalisable theoretical development. At the same time, when prison officers were included in the sociology of prison genre, they were typically presented in uncomplimentary stereotypical images. Even the literature that was crucial in the original development of theories of prison culture and its organisation did little to dispel such presentations. For example, Sykes (1958) viewed prison officers as 'corrupt'; Clemmer (1940), while not emphasising individual culpability as blatantly as Sykes, reinforced the image of 'disciplinarian'; and Morris and Morris (1963) did little to remove the image of 'harsh disciplinarian' and were less forgiving than Clemmer by intimating that it was the individual characteristics of prison officers that were central to this negative disposition: they were by nature 'bad' people. Jacobs (1983) concluded that subsequent commentators on the officer world reinforced the assumption that officers were "incompetent and psychologically, morally, and socially inferior” (p. 133) to the average person. Even more recent authors do little to refute such stereotypes. For example, the impression one gains from Parenti (1999) is that all officers are sexist, racist and constantly involved in sadistic violent games with inmates. 
There was also a tendency to demonise the prison officer as captor as opposed to the romanticisation of the inmate as captive. Consider the assertion from Clemmer (1940, p. 158), that "[t]he student of social science does not hate a stupid, brutal guard, just as he does not hate the feebleminded rapist” (my emphasis). Similarly, the image from Wicker, as cited in Cullen, Link, Cullen and Wolfe (1990, p. 90):

[C]orrectional officers face a life of nagging terror and work in an atmosphere where even good family men and churchgoers can be corrupted into physical brutality. \{my emphasis\}

What is interesting about the latter is the reference to the adverse conditions within which prison officers work. Yet, within most of the sociological literature, staff were disassociated from the well known pains inmates face even though officers spent a considerable portion of their day within the same negative environment that impinges on the well-being of inmates. In effect, there is a good deal of literature on the 'pains of imprisonment' for inmates; very much less on the 'pains of occupation' for prison officers. The negative consequences of the prison environment on non-inmate members of the community were simply unproblematic. Officers were able to withdraw from that environment when their shift ended, and therefore, if there were pains, these could then be left behind. However, as I demonstrate in this thesis, this delineation between inside and outside worlds for prison officers has been far more problematic: the culture of the job was, and still is in New Zealand despite changes brought about by restructuring, that one does not forget the prison as each shift finishes (see also Crawley, 2004).

There has been some examination of the effect of the prison environment on officers. However, rather than a sociological approach assessing the response from officers as a group as has been the primary focus with inmates, analysis tends to derive from psychological perspectives and centres on individual pathology or weakness (see for example Cheek \& Miller, 1983; Launay \& Fielding, 1989; Webster et al., 1983; Whitehead \& Lindquist, 1986). While I would encourage investigation into the pains of occupation experienced by officers, maintaining this on an individualised basis merely contributes to the existing demarcation, involving the romanticisation of inmates and demonising of officers. 
Coinciding with the above 'individualised' focus, there has been a tendency to examine prison officers during atypical circumstances and conditions. Hence we see a proliferation of research following general disturbances within the system, riots, high profile escapes, assaults and so on (see for example Adams, 1992; Marquart \& Crouch, 1985; Pogrebin, 1980; Scraton, Sim, \& Skidmore, 1988; Stotland, 1976). This has also been problematic in developing understandings of prison officers and the reality of their work because the resulting image detracted from the daily reality of the prison and prison work and situated officers as in a continuous struggle of brutality and intrigue. As I demonstrate in this thesis, however, the unremitting banality and monotony of prison life is much more usual.

At the same time, the literature demonstrated, as recognised as far back as Sykes (1958) but more explicitly and recently within Sparks, Bottoms and Hay (1996), that prison officers not only sought, but also actively negotiated a peaceful working environment (see also Crawley, 2004; Liebling \& Price, 2001). Indeed, the inference was that much of the work of prison officers was to do with the avoidance of 'trouble' rather than actively seeking, contributing to or participating in it. In other words, most of the research to date on prison officers and their work still does not capture the mundane reality of their work in the prison where violence and trouble was a possibility, but one which for the most part, did not usually intrude on its everyday ordinariness and routinisation.

However, more recent research, particularly Liebling and Price (2001) and Crawley (2004), have set in motion a more focused analysis of officers and their day-to-day duties. Liebling and Price contributed significantly to our understanding of the modern prison officer, being the first to highlight the contribution officers made to prison life distinct from the inmates' experience. They provide an extensive analysis of staff-prisoner relations with recognition that their role is pivotal to our understanding of prison life. They expose the ordinariness of routine duties, even if this is embedded in a focus on the complexities of the role of the modern prison officer. By doing this, they perpetuate the 'problem of role' rather than examining a 'problem of meaning', that is to say, what it actually is to 'be' a prison officer and 'perform' in this way. As such, a significant weakness of their account is that their analysis was devoid of the influences of occupational culture on them and 
concentrated only on the positive aspects of prison work rather than being able to provide a more balanced examination which necessarily touches on the 'dark side'.

Thereafter, Crawley (2004) opened, for the first time, some of the hidden truths of prison work, such as the complexity of emotion management necessarily performed by officers in response to inmate suicide and dissent. In this way, rather than a simple account of officers being cold, brutalising or disinterested (see Currie, 1989; Fitzgerald \& Sim, 1982), Crawley uncovers the humanity of prison officers, dispelling the stereotypes typically found in the literature, such as that of the stupid brute (see Clemmer, 1940). She also makes the important point that prisons themselves have their own unique working culture and that this can change, thus pointing to the possibility of divergence and differences in groups of officers' understandings and working experiences. Under such conditions there is likely to be increased stress, damaged morale and a discontented working group. Indeed, these same themes are central to my own thesis. One of the effects of restructuring was to destabilise this previously taken for granted aspect of the job and create role dilemmas for old style prison officers and new ones joining the service.

The final criticism I wish to raise here regarding the stereotypical analysis of prison officers within the prison research literature is their place as 'superordinates' or presiding members of the prison community. Their subordinate position relative to management has rarely been recognised. For example Sykes (1958) interchanged the terms officials, administrators, guards and custodians and only used the term 'governor' or 'warden' in reference to specific individuals. I would contend that this is not an issue of mere semantics, but instead is a masking of power relations. But where reference to differing power relations is raised there has been a tendency to minimise such influence. For example Goffman (1961) mentions, albeit only once and without elaboration, internal superordinates within the staff group. I would contend that just as the inmates' position relative to officers has been a major issue, so too should be the differential power relations between prison officers and the multiple levels of management which governs prison and prison life. 


\section{(iii) A Preference For Maximum Security Research Sites}

Much prison research has been conducted in high security type institutions (see for example Clemmer, 1940; Kauffman, 1988; Morris \& Morris, 1963; Owen, 1988; Sykes, 1958). Authors such as Mathiesen (1965) proposed that this emphasis was a consequence of an over-generalisation tendency in sociological prison studies whereby interest has been governed by a preoccupation with establishing commonality across institutions rather than looking for differences. I would contend, however, that the motives of researchers were far more pragmatic and directed at the expediency offered by such facilities. That is, due to their 'extreme' nature, they provided a concentration of investigated effects that could be elucidated quickly. The result has been a reification or normalisation of the maximum security experience. Where different facility types have been included in the analysis ${ }^{17}$, whether as a means of comparative or as exclusive research sites, the maximum, or highly custodial, institution has remained positioned as the normative prison and an 'extraordinariness' attributed to other institution types (see for example Dilulio, 1987; Genders \& Player 1989; Jones \& Cornes, 1977; King \& McDermott, 1995).

Normalising the maximum security experience has been problematic in developing a body of sociological knowledge of the prison given that maximum security facilities represent only a small minority of institution types. Of the seventeen male prisons in New Zealand, only one is classified as maximum security. The continued use of specialised type institutions for comparison purposes has similarly limited our perception of the prison experience as they also represented the atypical (see for example Genders \& Player, 1995). The result is a genre replete with atypical versus atypical and devoid of the ordinary. The more typical prison experience, namely the minimum to medium security facility has not been given anything like as much attention.

The presentation of prison and prison life developed by this predilection for focusing on inmates within maximum security facilities is that of an extreme environment that

\footnotetext{
${ }^{17}$ Such non-maximum security sites have tended to be treatment or programme-based facilities and objectives of research have been guided by their apparent selection criterion.
} 
abounds with tension and excitement. As an example, DiIulio (1987, pp 1-2) described his impressions of Walpole Prison in the following manner:

Inmates roamed about virtually unimpeded, glaring, making threatening gestures, often shouting profanities at the officers.... Officers wearing rubber boots and carrying shovels waded ankle-deep into the mess and were showered with insults and debris and human excrement.

Such academic accounts resonate with those within more anecdotal literature (see for example Boyle, 1977; Conover, 2000). While it may be reasonable to conclude that, at least for maximum security institutions, life can indeed be 'extreme', it is not justifiable to extend this understanding to lower security facilities which simply by their status suggest something lower was likely to be the norm. In these respects, this research was developed to re-construct the presentation of prison officers and their work within the context of the typical prison experience, that of the minimum medium security institution ${ }^{18}$.

\section{The Influence of Erving Goffman}

The mechanics of the research process I chose to investigate the way in which prison officers were affected by the changes in an 'ordinary' prison are set out in chapter two. The way I understood the social dynamics within the prison was greatly informed by the work of Erving Goffman, three of his books particularly. These are:

\footnotetext{
${ }^{18}$ The following provides a brief definition, according to the Department of Corrections (2004a), of security classifications as they apply to the New Zealand inmate security classification system. Maximum security inmates are those of high risk of escape and/ or public harm and/ or internal risk. They require an intensive level of supervision and should be separated from the mainstream prison population. High medium security inmates are those inmates of high risk of escape with a moderate to high risk to the public and/ or internal risk. These inmates require a high level of supervision. Low medium security inmates are those with a low level of internal risk and escape. The distinction between high and low medium security status impacts on certain procedures, such as the number of officers required for an escort or movement, however, a medium security facility will contain either. Minimum security inmates are of minimal risk of escape, low internal risk and low risk to public safety. Thus, they require only a low level of supervision.
} 


\section{(i) The Presentation Of Self In Everyday Life (1959)}

For Goffman, the world is a stage in which everyday life has to be 'acted out'. The roles we perform in this unending series of plays and dramas have to be carefully scripted and learned if everyday interaction is to be completed successfully. It is as if we are merely actors on this stage, with the parts we play shaping our characters rather than our characters being determined by natural attributes. In this book he demonstrates that the seemingly taken for granted way we conduct our life is not as taken for granted as it may appear. He argued that when an individual appears before others, they are motivated to control the impression that the other people will receive regarding the situation. This book is about the common techniques that people use and the contingencies associated with these techniques.

During an encounter with another person, an individual (actor) will adopt a social role and perform a pre-established pattern of action or routine. During this performance a definition or impression of the situation is portrayed which guides the interaction. For example, when an officer is interacting with an inmate, the officer will adopt the social role of superordinate which guides the inmate to adopt the social role of the subordinate, the interaction will then follow a pre-established pattern of this type of dynamic. The initial definitions of the situation projected by an individual tend to provide the plan for cooperative activity. The definition of the situation is portrayed in both the personal front of the performer and the verbal message given. As an example of a guiding verbal message, officers in my research varied the terms they used, such as 'hut/ house' or 'cell' to delineate familiar tasks from officials tasks. Use of the wrong term led to a confused interaction and the encounter would break down. Notwithstanding the importance of this, the verbal message is considered controllable and therefore easily manipulated. Thus an observer will use non-verbal cues to validate the impression that is being fostered. The primary non-verbal cue is personal front (and associated to this is sign-equipment). Personal front includes the person's manner and appearance: posture, speech patterns, facial expressions, bodily gestures, gait and sign-equipment such as insignia. Therefore, the importance of the way individual officers 'carry themselves' becomes an essential aspect of the presentation of self in prison. As I repeatedly observed in my research, wearing the uniform itself 
carried authority but at the same time the bearing of the particular officer (straight back, military posture, jangling keys, stoic facial expression, considered eye contact and so on) became another sign of difference between themselves and inmates, another sign of their power over them. Conversely, the inmates' bearing reflected their subordinate role (scruffy 'civvies', slouched posture, eyes to the ground and so on). Observers notice these non-verbal cues and then apply previous experiences with such individuals, acting roughly the same to the stereotype presented. Accordingly, an importance is placed on the familiarity of symbols as a way of gaining recognition and acceptance and anchoring interactions.

Goffman delineated between the regions in which actors perform. The 'front region' (or front stage) is the area in which an audience is visually or aurally present. While in the front stage, in order to foster a particular impression, actors expressively accentuate some aspects of a task while other aspects are suppressed. Officers most noticeably did this with regard to security checks. If it is known by both the actors and the audience that a particular task is required, actors will accentuate their activities (such as the officers rattling and pulling hard on grills) so that the audience is conscious of the performance. As officers prioritised security themselves, but at the same time needed to portray the appearance that they were approachable for case management tasks, they would suppress the appearance of non-scheduled security checks while seemingly performing other tasks (such as leaning subtly on the grills enjoying the sun but actually testing their veracity).

Conversely, the 'back stage' is the region away from the audience. For officers this was generally the staff rooms. It is in the back region that actors can relax, be schooled on the performance, practice the performance and so on. Further, in the back stage actors can freely communicate out of character as they are with others 'in the know' about the nature of their performance. Commonly, when actors are back stage they will derogate or praise their audience in ways that would not be possible in their presence. Derogation of the audience serves to maintain the solidarity of the group by enhancing the perception of the in-group-out-group split. This is often performed as mock role-plays of the audience or uncomplimentary terms of reference. This was frequently witnessed during the course of this research. Similarly, actors will refer to their routine cynically or technically to demonstrate that they do not take the same 
view of their activity as they maintain for their audience. Interestingly, with the introduction of case management, officers and inmates were now required to work together and hence developed their own back stage regions. For example, a case officer and inmate may perform a particular routine when others were present, but when sitting alone in the inmate's hut they would both perform out of character as this area had taken on a back stage region for them. In these situations, I would hear the officer and inmate acting cynically about their routine, such as an officer telling an inmate to attend a rehabilitation course 'just so it looks good for the parole board'. In these back stage regions, officers were in a difficult position as their behaviour was constrained in that they could not act fully out of character, such as derogating other prison officers, as officers remained their primary group.

The importance of this book for prison ethnography is his demonstration that the social world is not as taken for granted as it may appear. Thus, in everyday life, actors, such as prison officers, have to learn appropriate scripts, use personal front and sign-equipment for impression management and delineate their activity between different regions. An importance is given to particular aspects of the job, like wearing a uniform and the military insignia (lapels and epaulettes) as these are designed to give off important signals to the other inhabitants of the prison: the prisoners, who saw the uniform as an essential sign of the difference between themselves and officers; and other officers, for whom it constituted a sign of security and solidarity. At the same time, the importance of the way individual officers 'carry themselves' becomes another essential aspects of the presentation of self in prison. As this thesis will show, scripts, fronts, sign-equipment and regions have all become problematic in the aftermath of the dramatic structural reorganisation of the 1990s.

\section{(ii) Asylums (1961)}

The central concern of this book was to develop a sociological version of the structure of self and the adjustments people make within total institutions. Goffman defined total institutions as "a place of residence and work where a large number of likesituated individuals, cut off from the wider society for an appreciable period of time, 
together lead an enclosed, formally administered round of life” (p. 11). Hence the prison is a total institution. He was not concerned with the specific acts of individuals or their internal motivations, but rather the significance of their behaviour as it relates to the dynamics of the social structure. It is this explanatory power of the social structure that helps provide an account for the behaviour of officers within this research rather than any natural predispositions they might bring to the job. While the world was the stage in Presentation of Self in Everyday Life, here he is looking at the social dynamics and interaction that takes place on the specific stage of the total institution. Goffman provided some discussion of staff as superordinates, but only in relation to their structural association with subordinates. In this book he did not afford staff the same rigorous explanatory analysis as he did for inmates, as he said "[t]he main focus is on the world of the inmate, not the world of the staff” (p. 11). Nevertheless, he does introduce us to the complexities of the social dynamics of the prison. In particular, what going to prison 'does' to people. I contend that many of the same social dynamics that operate on inmates operate on officers, making Goffman's thesis, with extension, pertinent to understanding prison officers' experience of their work.

When an individual becomes a member of a total institution "[h]e begins some radical shifts in his moral career, a career composed of the progressive changes that occur in the beliefs that he has concerning himself and significant others “ (p. 24; original emphasis). It is my view that for both inmates and officers, a 'mortification of the self' (cessation of original self and replacement with an institutional self) takes place. The process occurs through a series of 'degradation ceremonies'. Of relevance for officers from my research were: 'the welcome' (where all members of the community demonstrated to the new officer that they have a low status, even amongst their low status group, such as being referred to as 'proby'); being stripped of their 'identity kit' (not being able to dress as they please, such as having to wear a uniform and not being able to wear makeup; having to appear a certain way which for some means getting a haircut not of their choosing); physical indignities (such as having to ask others before leaving their post to go to the bathroom; having to adopt an authoritarian stance); indignities of treatment by others (such as verbal and gestural profanities, obscene names and being ignored until one has proved oneself); engagement in activities whose symbolic implications were incompatible with their conception of self (such as 
having to strip search inmates and discipline grown men); violation of territories (violation of one's informational preserve regarding the self - both officers and inmates gathered indiscriminate information about each other; the self was openly expressed to others by others - nothing was sacred to the self; contaminative exposure - officers were forced into contact with inmates and inhabited the same contaminated environment that impacted on inmates); and desegregation of conduct (all conduct was under scrutiny at all times and activity in one area of the prison was passed onto all other areas making the self fully exposed). A new prison self, for both inmates and officers, has to emerge if one is going to survive in this strange new environment.

Goffman distinguishes between two types of adjustments that the individual makes to institutional life. 'Primary adjustments' are where the individual accepts what has been prescribed from the institution in regards to their role and their sense of self. Such adjustments provide institutional stability. Individual members may sustain primary adjustments due to satisfaction with these arrangements or an inability to build a different world through 'secondary adjustments'. Secondary adjustments are “any habitual arrangement by which a member of an organization employs unauthorized means, or obtains unauthorized ends, or both, thus getting around the organizations assumptions as to what he should do and get and hence what he should be” (p. 172). Goffman did not directly analyse the secondary adjustments of staff, although he did indicate that low level staff within an institution, such as prison officers, typically have less commitment and attachment to the organisation and thus would engage in more secondary adjustment behaviour than higher placed members. Nevertheless, he did locate them as being in cooperation with inmates to sustain their secondary adjustments. He argued that staff cooperated with the inmates' 'underlife', permitting certain violations, in order to reassert some control in the face of secondary adjustments. My thesis will demonstrate that staff, at least at this New Zealand prison, had their own institutional underlife as a means of redefining their social world and that this was structurally based as a means for them all to escape the banal 'reality' imposed by the institution and society for their lowly status and dirty work. 
The nature of the secondary adjustments that prison officers at my research institution employed was "contained, ${ }^{19}$. Contained secondary adjustments "[f]it into existing institutional structures without introducing pressure for radical change, and which can, in fact, have the obvious function of deflecting efforts that might otherwise be disruptive” (p. 180). Goffman's concept of secondary adjustments aids our understanding of many aspects of officers' behaviour. For example, he highlighted that behaviours that link workers to the institution during off-duty hours, such as officers attending to inmate recreational activities, demonstrates an over-commitment/ attachment type of secondary adjustment. Also, as found in this research, ex-military officers find it much easier to adapt to prison life. Goffman indicates that this is because secondary adjustments to an institution are an alignment that is already second nature to such workers rather than a shift in moral career. 'Working the system' was another form of secondary adjustment that officers within this research frequently employed. This requires intimate knowledge of the processes and systems of the organisation and an ability to circumvent these. As an example, during night watch shifts officers would partake in numerous unauthorised activities for unauthorised ends, such as bringing in a television to watch movies in the guard room or taking turns at sleeping in a back room. To avoid detection by the patrolling officer, each unit would elicit from the patrolling officer his next unit to visit and telephone the staff there to warn them of the impending inspection (thereby alerting the upcoming unit's staff to wake the sleeping officer or hide the television). Similarly, officers from this research would engage in 'make-do' activities. That is, using available artefacts in a manner and for an end not officially intended. For example, security radios were frequently used for entertainment rather than pure passage of information. Entertainment was a common end for officers' secondary adjustments at my research institution. As Goffman noted, within a total institution time needs to be marked. It is this sense of dead time that places a premium on 'removal activities' for members. In my prison this often involved games (board games, gambling, cards and so on) or sports (playing touch rugby, weight training and so on), often with inmates. As Goffman said, "[i]f the ordinary activities in total institutions can be said to torture time, these activities mercifully kill it” (p. 67).

\footnotetext{
${ }^{19}$ The other type of secondary adjustments is referred to as 'disruptive', whereby "the realistic intentions of the participants are to abandon the organization or radically alter its structure, in either case leading to a rupture in the smooth operation of the organization” (Goffman, 1961, p. 180).
} 
Irrespective of the benefits that can be achieved from secondary adjustments, some are merely performed because it is forbidden. For example, officers at my research institution went to great lengths to ensure that meals from the inmates' rations were available for staff. Although Goffman refers to staff eating institutional meals as a common and minor secondary adjustment with a perquisite status, given the lengths of secrecy involved in this activity I would argue that it was also a reflection of disregard to the social world imposed as typically the food was of such a low quality that it is doubtful it would be sought by persons able to access alternate food and thus it constituted a rejection of the rules rather than a genuine perquisite. The importance and ritual placed on such trivial things as food, demonstrates another feature of Goffman's thesis - how the minutiae take on more significance within total institutions. I would contend that this does not just apply to inmates and their lack of access to goods and services. Officers equally alter their value of things in a similar manner to inmates. For example, even when outside of the prison, officers from my research would gamble with each other using chocolate bars ${ }^{20}$ (as is done in prison) rather than money.

Of the 'modes of adaption' Goffman outlined that individuals employ to manage the tension between the outside and inside worlds, 'situational withdrawal', 'colonisation' and 'playing it cool' were applicable to my officers. For inmates, situational withdrawal occurs when they intentionally challenge the institution by refusing to cooperate with staff. For staff, this is expressed as an attempt to increase the social distance between themselves and the character imposed by the institution. For example, some officers, at my research institution, would request transfer to the noncustodial unit or low inmate contact tasks (such as night watch) to increase the social distance between themselves and inmates. Alternatively, if they were seeking social distance from fellow officers, they would endeavour to secure the daily tasks that removed them from contact with other officers (such as taking inmates out of the institution on work assignments or transferring inmates to prisons outside of the region). Colonisation involves the community member using the outside world as a point of reference to demonstrate their preference for life within the institution.

\footnotetext{
${ }^{20}$ The common luxury item to be used as a betting commodity by inmates within the prison was the peanut slab. The peanut slab is one of the cheapest chocolate bars available to purchase. Officers would similarly use the peanut slab as a commodity, even though they were not financially limited in their options as inmates were.
} 
Officers at my institution, particularly longer serving staff, would frequently make reference to alternative employment and life style options as less desirable than their current situation. Playing it cool is a combination of secondary adjustments, colonisation and loyalty to the group. This mode of adaption for staff at my prison was increasing in proportions due to the anxiety and stress of the structural and group demographic changes that were occurring. These members reduced contact with their peers, volunteered for nothing and cut their ties with the outside world sufficiently to give a cultural reality to their inside performance but not enough to lead to colonisation. I refer to these officers in this thesis as 'functionaries'. They did their job, joined in as much as necessary, but tried to remove themselves from the complete submersion into the occupational culture that existed for some officers and was more dominant in the past. Such members provided stability to the performance, but not authenticity. Colonised officers, the stereotypical officer from the literature, found those that situationally withdraw or played it cool difficult to categorise as they were not true members in their minds.

The importance of this book for prison ethnography is his analysis and explanation of the complexities of the social dynamics of the prison and the way in which participants' lives are institutionally 'created'. Thus, the minutiae of institutional life takes on specific meaning that is defined by the context in which it takes place, such as: making allegiances; the remarkable importance attached to daily rituals and everyday items; institutional 'lingo' (argot); resistance (development of secondary adjustments and an organisational underlife); and the emergence of a 'prison self' to cope with these arrangements. This is not limited to inmates, but with extension, provides an understanding of prison officers.

\section{(iii) Stigma (1968)}

In this book, Goffman provides an account of the effects of stigma and the need for strategies of self-protection, such as passing, covering, and the in-group alignment of like-situated individuals. Although Goffman does not analyse the stigmatic moral career development of persons due to occupation, his discussion of inmates has 
striking parallels to the experience of my prison officers. Hence, I have extended his analysis to include this group. For example, he proposed that inmates learn about their stigma through intimate contact with other inmates in the process of being transformed into being one of the 'fellow-afflicted'. I would contend that this also applied to my officers as they did not consider the effects the stigma of their occupation would have on them until they were in intimate contact with fellow officers. Thus, his thesis has importance to prison ethnography in that, with extension, it allows us to appreciate the sense of shame felt by officers about their 'dirty' occupation, and the way in which this simultaneously cut them off from life outside the prison and bound them closer to it: prison became the only place where they did feel accepted. It was their occupational culture that provided the means of making sense of their 'stigmatised' selves and their 'stigmatised' work.

The first central tenet of his thesis relates to social identity. He asserted that when we meet a stranger we use their general appearance to create a 'social identity'. This social identity encompasses normative expectations and social demands. From this we impute a character upon the individual which constitutes their 'virtual social identity'. Subsequently, the category and attributes the individual proves to possess constitutes their 'actual social identity'. Of importance, then, is whether there is a discrepancy between the virtual social identity that we impute and the actual social identity that the individual is revealed to have. When a stranger possesses an attribute that makes them deleteriously different to others in their category (and hence to the character we have imputed and the associated normative expectations), the individual is considered tainted, their social identity is spoiled and this discrepant attribute is their 'stigma'. For Goffman, stigma is any "attribute that is deeply discrediting" (p. 13). A stigmatised individual is well aware of their stigma attributes. This awareness was evident within my group of officers in the way that they exaggerated their stereotypical attributes during back stage performances and by their black humour reference to each other as 'screws'.

Of the three types of stigma Goffman discusses, 'blemishes of individual character' was particularly relevant for my officers. Their character was considered problematic as they chose to work with 'human garbage'. Goffman asserts that people tend to attach numerous additional attributes to the original one. Thus, a variety of 
stereotypical negative attributes were imputed on prison officers due to the supposed traits of such workers; such as being domineering, authoritative and unintelligent brutes. Therefore, upon identification as an officer, the officer's actual social identity was revealed as tainted. Goffman proposed that stigmatised persons tend to hold the societal belief about their identity. This was evidenced in my group of officers by their generalised reluctance to tell non-prison people what they did for a job. Although a stigmatised person may feel they should be treated normally, they are aware that others do not accept them on 'equal grounds'. Possessing this societal view would make it difficult for officers to make sense of their work as meaningful, as to society it was dirty work, something 'normal' people would not choose to do. This was where the officers' occupational culture was most useful. Their occupational culture acted to insulate them from some of the effects of the stigma by imputing a different set of identity beliefs. Thus, they bore the stigma of their occupation but it did not impress fully on them as their culture provided an alternative set of attribute understandings. They were then able to make sense of their work as meaningful. Goffman proposed that when a group rejects the societal norms in this way, they often consider themselves better than 'normals' and that their lives are better than they otherwise would be if they were to be a normal. There were certainly recurring comments to me by my group of officers that indicated this belief, that 'what they did counts' and they were 'something special' (indeed, such ideas are reflected in the Department of Corrections advertisement in Figure 1.5).

The second central tenet of this book is the distinction between the situation and behaviours of those that are already 'discredited' compared to those that are 'discreditable'. If one is discredited their stigma is known. The discredited person is thereby confronted with managing the social tension arising from their known stigma during social encounters. Alternatively, if one is discreditable their stigma is not known, but could be revealed during social encounters. Discreditable persons are confronted with the tension of information management to prevent their stigma becoming known. The two common techniques employed by discreditable persons when interacting with normals are to attempt to 'pass' as normal, or to 'cover' their stigmatic attribute. Goffman suggested that how the stigmatised person behaves during social encounters is linked to their moral career development. That is, discreditable individuals generally move from passing to covering, then within 
covering they will openly discredit themselves and use advanced covering strategies to lessen the resulting tension.

For my officers, during encounters outside the prison, their status was generally discreditable. Their uniform was their primary 'stigma symbol' as without it, their stigma was not readily identifiable. This accounts in part for their prompt removal of their uniform after shift (often before leaving the grounds). After time, however, multiple stigma symbols are unwittingly adopted by a person, such as particular phraseology, physical stance and so on. These additional symbols can be more difficult to mask, conceal or pass - increasing the risk of 'discovery' and hence tension during an encounter. Goffman highlighted that uncertainty and anxiety arises when the stigmatised encounters a normal leading to unanchored interactions and unstable communication. This awkwardness is felt by both the stigmatised and the normal. Indeed, the anticipation of contact with normals can lead some stigmatised individuals to rearrange their life to avoid it. My officers displayed this behaviour, such as preferring to live in areas close to the prison exclusively occupied by fellow officers. Such behaviour may be an attempt by the individual or group to reject their rejecters; or it may simply be a means to avoid the anxiety inducing interaction. Regardless, for my officers, their social circles became increasingly smaller until it was limited to like-situated others.

The importance of this book to prison ethnography is that it allows us to appreciate the sense of shame felt by officers about the stigmatic effects of their 'dirty' occupation and how this simultaneously binds them closer to the prison while cutting off the outside world.

More generally, Goffman's dramaturgical perspective removes the taken for granted assumptions we may have of the prison as a work environment. Rather than a group of individuals with various idiosyncratic attributes, officers are a stigmatised team of actors within a specific set of dramas carried out on the total institution stage of the prison. Goffman's analysis allows us to locate officers as a product of their social structure and arrangements. As such, one would expect that the dramatic structural changes of the 1990s would have a significant effect on their concept of self and ability to make sense of their life and work in their changing social world. 


\section{Thesis Structure}

\section{Chapter Two: Doing Prison Research}

This chapter outlines the methodology involved in this research: the triangulation of a predominantly quantitative questionnaire, semi-participant observation and in-depth semi-structured interviews. It also discusses some of the practical problems one is confronted with when undertaking prison research and the pressures of and challenges associated with 'going native'.

\section{Chapter Three: The Historical Development of The New Zealand Prison Service}

This chapter examines the historical development of the New Zealand prison service from the late nineteenth century to the structural changes of the early 1990s. Post 1984 economic restructuring altered the nature and character of the public service. It produced profound quantitative and qualitative changes within the prison environment and led to increased divisions amongst the officer body. The previously known social and power arrangements had been unchallengeable and straightforward - but these were to become increasingly situational and contestable. Indeed, within a short period of time the stage on which prison officers enacted their well-rehearsed script was to collapse. After significant challenge by the officers, they faced defeat. It was this fractious stage upon which the research was conducted.

\section{Chapter Four: The Setting For This Research And The People Involved}

This chapter examines the setting and demographic backgrounds and details of the prison officers at the target institution and compares these to the findings of international research. The main differences in the New Zealand context related to a comparatively high complement of ethnic officers and disproportionately low levels of length of service, factors which were to become of prime importance in the 
reorganisation that was taking place and the effects it produced. The target prison had divided into two 'jails', each with their own characteristics and identities - attracting different types of officers and contributing to the erosion of homogeneity and unity within the officer body.

\section{Chapter Five: Learning The Scripts}

Some of the immediate consequences of this erosion of the homogeneity and unity of the officer group were reflected in the training of new recruits. This chapter examines the increasingly complex process of 'becoming' a prison officer. It had become complex in the aftermath of the policy changes for two reasons. Firstly, due to insufficient numbers of, and unwillingness by, senior officers, probationary officers were increasingly being paired with inexperienced induction officers leading to inconsistent and inaccurate advice being given on how to appropriately behave. Secondly, with the imposed Head Office script on top of the traditional officers' script, there was no longer one prison officer 'script' to learn. As different groups of officers accepted and rejected various aspects of the two, a multitude of potential scripts emerged. Each new recruit, indeed all officers, had to at least be familiar with all variations if their behaviour, and that of others whom they were interacting with, was to be known and predictable. For longer service officers, frustration with and lack of trust in the new recruits led to withdrawal from the officer group.

\section{Chapter Six: Handling Your Lag}

While prison officers had previously been able to buttress the pains of their occupation through their traditional occupational culture, the changes in the prison service, whilst having been written into their scripts, now contributed to their pains. Thus, with the increased homogeneity, decreased unity and a weakened occupational culture, handling their lag had become an increasingly contentious and difficult matter. This chapter examines the complexities of the job, for all officers, in the aftermath of restructuring. In particular, the increasingly rigid line between officers and management as antagonism and resentment rose; and the increasingly fluid line 
between officers and inmates with the injection of case management to their scripts. This constantly shifting relational line made managing inmates contentious, increasing stress and uncertainty within the officer group. On top of this, the injection of ethnic males and women officers was rewriting their scripts in unexpected ways women producing little effect, while ethnic culture usurped the traditional officer set.

\section{Chapter Seven: Unit Divisions}

The reorganisation of the prison service had indeed successfully weakened the traditional prison officer culture, homogeneity and cohesion. Unit management was to cement this achievement and prevent the re-emergence of the unity of old. This chapter extends chapter four's analysis of the prison officer group's demography, demonstrating that the different demographic structures evident between the two jails became even more polarised at the unit level. The officers, through selective transfers, had manipulated the unit divisions. As a result of the division of the prison into units, acculturation into the wider officer group had become problematic. Unit management created problems far more insidious than those which it had intended to remove as the units began to develop their own ways of functioning, which were often at odds with the aims and expectations of the prison authorities.

\section{Chapter Eight: Each Day Is Different?}

The changes imposed on the prison service during the early 1990s were now a reality and had to be accommodated by all prison officers, regardless of how painful this was to them. This chapter illustrates how the majority of officers were becoming increasingly individualised and 'functionary' in their approach to their work and colleagues. It outlines the five daily strategies of prison work that combine the promise of excitement and challenge with the traditionally required routine of predictability: dealing with the daily grind; making the tedious fun; making the minutiae count; making the boring exciting; and making the exciting mundane. Such strategies allowed the prison officers to simultaneously claim that each day was different, while each day actually remained the same. At the same time, however, 
each day was indeed becoming more different and less predictable. This was the reality that prison officers were contending with, without a solid occupational culture to support them. The conclusion of the thesis assesses the impact of superimposing change on this organisation. 


\section{CHAPTER TWO: DOING PRISON RESEARCH}

\section{My Research Methods}

The central task of this thesis is to explore the day-to-day experiences of prison officers in one ordinary New Zealand prison as they attempted to make sense of their work in the aftermath of dramatic organisational change. Therefore, the methodology of this research incorporated an ethnographic design with a triangulation of quantitative and qualitative methods. A questionnaire, semi-participant observation ${ }^{21}$ and in-depth semi-structured interviews combined to produce the data for the subsequent chapters of this thesis. All field research was conducted at one New Zealand male minimum medium security penal institution over a sixteen month period beginning mid 1997 through to late 1998.

The process can be summarised as follows. One hundred and fifty two census style questionnaires were distributed representing the entire prison officer population of the target institution at the beginning phase of the research. An overall return rate of $72 \%$ was achieved. Demographically, the returnee population demonstrated reasonable comparability with the general population of officers at the target institution, and hence generalisation was permissible.

Approximately nine months was spent conducting the observation phase. A total of 178 formal eight-hour shifts were incorporated in the design, 35 familiarisation or

\footnotetext{
${ }^{21}$ The observation role that I took varied according to the situation. While in the prison and visible to non-prison officers (the official front stage of the research) I employed a predominantly non-participant observer role. Thus, I did not wear a uniform, carry keys or engage in official prison officer tasks. If the circumstances required, I did engage in some activities (such as making coffees). However, as I was neither an inmate nor officer I remained a 'researcher' and, therefore, formally a non-participant. While in the official backstage of the prison, such as the staff facility or guard room (only visible to prison officers) I was a participant observer joining in all unofficial activities. Similarly, while in the unofficial front and back stages (such as attending social functions) I was a full participant observer. In this way, a mixture of participant and non-participant observation techniques were used, hence the application of the Goffmanesque term 'semi-participant' observation.
} 
non-recorded shifts and 143 fully recorded shifts. An overall formal observation rate of $73 \%$ of officers was achieved representing 116 of the total 158 prison officers rostered during this phase of the research. Demographically, the observed population was highly comparable to the total population, permitting generalisation.

Two distinct sets of interviews were employed in this research. The first set of interviews, referred to as 'specialised', were conducted within two weeks of specified serious incidents within the prison that directly involved the interviewee officers. Sampling was achieved via self-selection and snowball techniques. A total of eight specialised interviews averaging two hours each were conducted.

For the second set of interviews, referred to as 'generalised', cluster purposive sampling was used to derive a deliberately skewed interviewee population to ensure reasonable representation of each demographic category. A total of 31 generalised interviews were conducted averaging two and a half hours each.

\section{Overview Of The Research Process}

Prior to any other negotiations, ethical approval was obtained from Victoria University of Wellington's Human Ethics Committee as per regulation requirements (see Appendix A). Full support was given and there were no requested adjustments to the proposed methodology. Dixon (1997) suggested that researchers using ethnographic methodologies typically encountered difficulties gaining support and approval from institutional ethics committees; however, this was not experienced in this application. During the course of the research a minor alteration to the proposal was resubmitted to the Committee for consideration and was promptly approved.

Following ethical approval there were negotiations with the Department of Corrections, which represented the governing body of New Zealand public penal institutions. The representative involved offered generalised support for the project 
and approval to approach the Regional Managers ${ }^{22}$ of the pilot and target institutions; however, it was at their discretion, along with the approval of the given Site Managers $^{23}$, whether their prisons would ultimately be involved. Informed consent was given verbally. The Department of Corrections neither offered input nor direction, nor placed stipulations or requirements on the project enabling the research to be conducted independently of them. The ease of negotiation stands in contrast to the experiences of other prison researchers who report unnecessary delays, attempts to contractually limit scope and so on (see for example Wacquant, 2002). The reason for the ease of the approval for my research remains unclear.

Access negotiations to the specific prisons were conducted separately and concurrently. The Regional Manager governing the pilot institution offered support and permitted contact with the Site Manager. Similarly, the Site Manager provided support and granted permission for entry to the site and introduction to a Unit Manager who facilitated the actual physical access within the prison. The Site Manager signed an informed consent form for formal access. The pilot study was then conducted (see further in this chapter).

Formal access to the target institution proceeded without difficulty with the Regional Manager offering generalised support and introduction to the Site Manager. A contract was negotiated between the Site Manager, my research supervisor and myself with reference to: confidentiality of information gathered; behaviour and responsibility for basic safety and security; and presentation of research findings (for a copy see Appendix B). In accordance with this contractual arrangement, I attended an induction session for Security Awareness for Non-Custodial Staff. Martin (2000) suggested that such a requirement prior to entry was standard practice.

\footnotetext{
${ }^{22}$ New Zealand Public Prisons Service has been divided into seven regions with two additional areas providing support to one of the regions (see Appendix F). Each region was to be self-contained both geographically and by providing a full range of services required to manage the local inmates from reception as remand to pre-release programmes and sentence release. Each region had a Regional Manager who was responsible for all prisons and directly related services within the region (see Appendix J). Each prison had a Site Manager responsible for the specific site and directly related services and who reported to the Regional Manager. Within each prison each unit had a Unit Manager who reported to the Site Manager.

${ }^{23}$ During the initial stages of the research project this position was referred to as 'General Manager'. However, this position was restructured and reassigned as 'Site Manager'. The latter term has been used for consistency in this thesis to denote this role. The term 'General Manager' then applied to the 'Site' Managers of the two area Prisons that were independent of, but supported, one of the regions.
} 
As with the Department of Corrections, neither input and direction nor stipulations and requirements were placed on the project by the Site Manager. Support was then offered with consent to be present on site. However, the inclusion of specific units was to be at the given Unit Manager's discretion. The Site Manager signed an informed consent form for formal access. As an independent initiative to facilitate information flow regarding the research, the Site Manager had an internal staff memorandum distributed throughout the institution (see Appendix D) ${ }^{24}$.

Unit Managers were formally presented with an overview of the project during a scheduled weekly meeting. Attendance and presentation of the project to staff at unit meetings were offered with several managers accepting and facilitating this process. All Unit Managers expressed interest and support for the project but were assured that specific consent would be sought prior to intended access to their unit. Each Unit Manager was approached individually for informal discussion regarding the project and to seek verbal consent for access one week prior to his or her unit's inclusion. No request for access was denied.

As a professional courtesy, information sheets were sent to the two separate Union Representatives ${ }^{25}$ on site along with a letter offering to meet and discuss any issues they wanted to raise about the project. Neither responded in an official capacity. Informal discussions with these representatives at a later date revealed they did not consider the research a union concern.

It was decided that one site was sufficient for research proper purposes as the selected site was comparatively large for New Zealand standards and provided both traditional wing style units and modern enclosed style units. The site was divided into two subsites. I have named these Alpha Jail and Beta Jail for identification purposes. I made

\footnotetext{
${ }^{24}$ Distribution of this document initially concerned my research supervisor and myself because of the potential association of the project with management (as opposed to it being perceived as an independent academic initiative). It was feared, given the closed nature of this community, that a malevolent bias of the data could result. These concerns were allayed after social/ informal access to the officer group was attained and it was evident that my independence was recognised.

${ }^{25}$ At the time of my research, the New Zealand prison service had two unions representing prison officers. The Public Service Association (PSA) was the original union. A renegade militant group separated during a period of industrial dissent to create the Prison Officers Association (POA). Staff elected to join one or other of the unions. The significance of two unions will be discussed in chapter three.
} 
this division for several reasons. First, treating the prison as having two separate jails replicated the division that the prison administrators, staff and inmates were familiar with both formally and informally. Secondly, it provided a natural grouping of data, as the 'two sites' were distinct in respect of unit style, setting, regime and architecture. That is, Alpha Jail represented the older style prison, built in the 1960s with medium maximum security classification regimes, whereas Beta Jail was the modern style prison with minimum security classification regimes in the form of sixty-bed chalet type units, enclosed with wire fencing and a gatehouse entry (the construction of these had begun in the early 1990s). Finally, it provided a logical progression through the units.

In brief, Alpha Jail contained three units, two of which housed inmates with the third unit of officers providing alternative services such as receiving and escorting inmates. Muster capacity was 112 and there were approximately 58 prison officers ${ }^{26}$. Alpha Jail design resembled a modified telegraph model, based on a roughly rectangular perimeter (see Appendix E). Beta Jail contained six units ${ }^{27}$, all of which housed inmates. Regular muster capacity was 330. However, this could be extended to 358 during periods of muster blowout. There were approximately 100 prison officers. Each unit was geographically distinct with only one set of two units physically, though not administratively, inter-connected. For the purposes of my research, each unit was randomly assigned a colour to represent its 'name'. As a quick reference guide see Table 2.1 below.

\footnotetext{
${ }^{26}$ These figures represented the numbers at the time of entry to each unit, however, staffing levels varied throughout the research period. Therefore, this figure is an indicator only and totals may not be exact in further discussion.

${ }^{27}$ Table 2.1 only shows four units in Beta Jail. At the time of my research there was an additional two units. One unit was excluded due to it having establishment issues and a further one was opened towards the end of the research period and therefore was not available for consideration in the design.
} 
Table 2.1

Target Institution's Division with Assigned Unit Names for Easy Reference During Discussion Sections

\begin{tabular}{cc}
\hline Alpha Jail & Beta Jail \\
\hline Yellow Unit & Pink Unit \\
Orange Unit & Lime Unit \\
Green Unit & Grey Unit \\
& Purple Unit \\
\hline
\end{tabular}

Once formal access had been granted, information sheets and posters were distributed and strategically placed on display throughout the institution. These sheets and posters were substantively similar, though varied in format to reflect the target recipients which constituted officer staff, non-officer staff, inmates and visitors to the institution. Supplies were regularly replenished during the course of the research.

Secure identifiable research boxes were placed in all guardrooms, staff rooms, the receiving office and public visitors' rooms for depositing written comments, questionnaire returns and request forms for the summary of results. These boxes were cleared regularly and resecured. Whilst these boxes provided some amusement for officers initially, none were tampered with in the course of the research.

The research proper then focused on Alpha Jail for several reasons. In the first instance, it afforded the most public display of my presence and hence facilitated awareness and interest in the project throughout the institution. As the centralised block, representatives from all officer groups and units were able to 'view' me and have informal discussions about the research, thereby initiating the social access process $^{28}$. As Alpha Jail contained the medium to maximum security inmates, social access was further influenced by the perception that, as I could conduct myself within that environment, I should equally succeed within minimum security units. Similarly, as the inmates from Alpha Jail progressed through to Beta Jail, they transferred

\footnotetext{
${ }^{28}$ The term social access is used here to distinguish formal access with informal (social) access. Formal access represented official permission to proceed with the research, whereas social access was the acceptance and support of the research by the actual participants and associated members of the prison community.
} 
information to other inmates about my role and purpose, thereby reducing the time required for units to adjust to my presence and resume regular behaviour. Finally, as the three units were geographically contained, it eased my personal transition into the research role.

A 'familiarisation' period was incorporated into the methodological design. This involved a week in each unit prior to the commencement of the research proper during which no official data was collected. This period was significant for several reasons. In the first instance it afforded officers an informal opportunity on shift to discuss the research with me and clarify any issues related to it. Similarly, I was able to learn details regarding the officer group such as names, relevant idiosyncrasies and generalised dynamics within the officer group, inmate group and between the two groups. Further, it enabled me to become familiar with the specific routines and systems of the given unit which facilitated note taking in the observation phase. It also provided an opportunity for officers to evaluate my effect on group dynamics, test my apparent allegiances and so on. Finally, as the presence of a young non-staff female was unusual within this environment, it provided time for inmates to adjust and the atmosphere and behaviour of the unit to settle prior to data collection.

The process of the familiarisation period varied for Alpha Jail and Beta Jail. For Alpha Jail this involved a one week period in each unit consecutively, followed by the observation phase in each unit separately. This approach was taken as I was new to the prison at this time and this permitted a generalised extended familiarisation period that was mutually beneficial for the participants and my own initiation into prison life.

However, given the geographical separation of Beta Jail units, the process as regards these differed in so far as the one week familiarisation period was directly followed by the observation phase. This was necessary as the time lapse would have been too significant in respect to movements and changes within the specific units, rendering the familiarisation period pointless. Further, at this time I was already becoming well known throughout the prison community and therefore my acceptance was accelerated. 
Upon completion of the familiarisation period in Alpha Jail, questionnaires were distributed to these units. The observation phase then commenced. Beta Jail questionnaires were not distributed until the end of the familiarisation period of the first Beta Jail unit as prior distribution would have then been ineffectual. Participants were neither sufficiently familiar with the research project nor the researcher.

Questionnaire collection officially terminated at the completion of the observation phase. No further returns were received. General interviews then commenced. Postincident interviews, otherwise referred to as specialised interviews, occurred within two weeks of the specified event to capture recency effects of the incident. Field data collection ceased approximately sixteen months after initial entry.

\section{The Questionnaire}

The purpose of the questionnaire was to gather predominantly quantitative data to establish the basic elements of the officer group (for a copy of the questionnaire see Appendix C). This data was to demonstrate the extent of the heterogeneity of the group, and to provide a basis on which to contextualise the subsequent qualitative data. The questionnaire also provided insights as to the 'typicality' of this officer group compared to that in other prison literature ${ }^{29}$.

The qualitative data sought from the final portion of the questionnaire was to initiate reflective thinking on the part of the officer in addition to affording the participant group an opportunity to highlight areas they considered important. It should be noted, however, that the research design and direction was clearly and deliberately not collaborative and remained independent. I wish to make this explicit as at times members of management, the officer group and inmates swayed between being subtle

\footnotetext{
${ }^{29}$ The questionnaire also had the serendipitous effect of establishing the project as academically based for the participant group as they were unfamiliar with the scientific validity of qualitative methods. That is, my authenticity as an academic researcher was likely to have been questioned had I begun with the observation phase, which was perceived as simply 'hanging out', or worse, aligned with a 'timeand-motion' audit. Further, it demonstrated the independence of the research as much of the requested information could be attained from staff files, however, use of the questionnaire explicitly pointed to the supposed unavailability of these files to the researcher. The Site Manager had offered access to files but I chose to use the questionnaire.
} 
to forceful in their 'suggestions' of data to be included or excluded. No consideration was given to such directives.

\section{The Pilot Questionnaire Process}

The questionnaire pilot was conducted within a region and prison that was comparable to the target institution in classification status, size and design. As a result of the pilot the question style was changed to make it more understandable to officers although the information sought remained much the same.

In the first instance, opportunistic sampling was employed, as population availability was dependent on shift patterns predetermined by the prison. Therefore, whoever was on shift and present on the particular wings during my time in that unit was considered a potential participant. Purposive semi-random sampling was then used to ensure a varied but targeted demographic set. Twelve usable data sets were sought including: two female; two male; two Maori; two Pacific Island; two less than one year service; and two greater than five years service. These categories necessarily overlapped.

Upon entering a unit I gave the rostered staff copies of the relevant information sheets, consent and questionnaire forms. We discussed the research and the majority of officers filled in the paperwork at that time and offered verbal feedback about the process and project. I then repeated this process in the next unit until all units at the prison had been included. Thirty one usable data sets, covering all sought demographic variations, were achieved. All officers approached participated.

Questionnaire data was coded and entered onto spread sheets for analysis. Comments, recommendations and answer style were surprisingly similar making adjustment to the final questionnaire reasonably unproblematic. As a result, a second pilot was not conducted. 


\section{The Questionnaire Proper Process}

Officers received a questionnaire pack in their individual mailboxes within each unit. I personally delivered these during night watch shifts to ensure accurate delivery and to minimise unit disturbance during this process. The questionnaire pack contained: an introductory letter with instructions on the questionnaire process; a general information sheet regarding the research; an information sheet about the questionnaire; two copies of the questionnaire consent form, one for their records and one to be returned with the questionnaire; a questionnaire; and a postage paid return envelope.

Two friendly reminder letters, accompanied by repeat questionnaire packs, were distributed to non-returning officers' mail boxes, the first approximately six weeks after initial receipt of the questionnaire pack and the second six weeks after the first reminder. Questionnaires were returned by hand to the researcher, via the postage paid envelopes and in the secure boxes throughout the institution.

Questionnaire responses were coded and entered onto excel spread sheets for analysis using SPSS. Original questionnaire forms were destroyed after data entry.

\section{Sampling frame.}

Census or complete sampling style was used. Thus all prison officers listed on unit rosters at the time of distribution, including those on leave or secondment, were included in the sample.

Although census sampling had been used, a method of combining data was required to make the volume of information more manageable. The categories selected were gender, ethnicity and length of service. These categorisations were then applied to all data sets throughout the research proper to ensure representativeness of subsequent sampling techniques and participation rates. The rationale behind these categories is as follows: 
(i) The gender of prison officers, particularly within male prisons, has frequently been raised in prison research literature as an important variable of analysis and therefore has been separated as a demographic category for this research. The literature indicated that gender was an important consideration for a number of reasons. For example, the differential tasks that may arise due to gender (see for example Bennett, 1995); perceived and experienced organisational barriers as a female employee in a non-traditional occupation (see for example Jurik, 1985a; Owen, 1985); negotiating stereotypes and varied expectations (see for example Hampton, 1993; Jurik, 1988; Jurik \& Halemba, 1984); male attitudes toward female co-workers within the prison environment (see for example Walters, 1993).

(ii) Ethnicity has similarly been highlighted as an important area of analysis. While the prison workforce in New Zealand had historically been white, male and rural, affirmative action practices had led to an increasingly heterogeneous mix of officers, with significant implications for prison officer culture (see for example Carroll, 1974). Conflict within the prison officer group had led to generalised allegations by white officers regarding differential practices by ethnic officers (see for example Jurik, 1985b; Klofas, 1986). Similarly, racial tension and conflicts had been identified as significant contributors to the uneasy social order within the prison community in international research (see for example Owen, 1985; Zimmer \& Jacobs, 1981). For these reasons, it was important to separate ethnicity as a demographic variable.

The reason for the three selected ethnic categories (European/ Pakeha, Maori and Pacific Island) requires explanation. The pilot questionnaire's ethnic identity question followed the standardised format used in the New Zealand Department of Justice Census 1991 (Braybrook \& Southey, 1992) ${ }^{30}$. Both written and verbal feedback from participants indicated this was unnecessarily complicated. While the Braybrook and Southey document provided a table of the complete demographic categories, for simplicity, when presenting ethnicity with other variables, they reduced the original twelve categories to four: New Zealand European; New Zealand Maori; Pacific Island; and Other.

\footnotetext{
${ }^{30}$ Subsequent Prison Census' documents used similar question format with revision only in accordance with National Census question style. To access subsequent Prison Census' refer to the Ministry of Justice rather than Department of Justice.
} 
American literature concerning ethnicity of prison officers typically distinguishes only between coloured and non-coloured with the occasional reference to other categories, such as Hispanic officers. However, in New Zealand, people of colour are identified according to ethnic affiliations as Maori or Pacific Island peoples. This is because there are important differences between the status, treatment and experiences of these two groups ${ }^{31}$. Hence this research distinguished the ethnic demographic categories of Maori and Pacific Island within the 'coloured' category.

The category of Pakeha is a unique indigenous New Zealand term that colloquially refers to white New Zealand citizens. Its use here refers to those participants who did not identify as either Maori or Pacific Island descent, were fair skinned and were predominantly New Zealand born, albeit with a small mix of other European cultures.

(iii) Length of service was divided into three categories (0-4 years, 4-10 years and $10+$ years) to take account of the experiences of important changes within the structure of New Zealand prisons and the prison service which had taken place since 1989 and which at the time of the research was undermining the traditional homogenous and long serving nature of this occupation.

\section{Participation rate.}

In total, 152 questionnaires were distributed throughout the target facility, 59 to Alpha Jail and 93 to Beta Jail ${ }^{32}$. The following tables represent the basic demographic structure of the two jails at the completion of each questionnaire phase and the complete prison in terms of a combination of the two data sets.

It must be noted that these population figures represent the questionnaire phase exclusively. Over the 16 month research period numerous factors influenced the constituency of the officer group, such as: transfers between units, transfers between Alpha and Beta Jails, transfers and secondments to external jails, resignations,

\footnotetext{
${ }^{31}$ In brief, Maori are the indigenous peoples of New Zealand (Tangata Whenua) while Pacific Island people may be first or second generation immigrants from the neighbouring Pacific Island countries.

${ }^{32}$ Note that of the 93 Beta Jail participants, 5 participants left the job during the collection phase. These numbers have been kept within the total figure even though response was neither anticipated nor received.
} 
terminations, new staff intakes and so on. For this reason, these figures do not identically reflect the figures presented for the following phases of research.

Table 2.2 demonstrates that the returnee population, that is, those who returned questionnaires, was reasonably comparable in the given demographic categories to the total population set of Alpha Jail.

Table 2.2

Demographic Details for the Alpha Jail Prison Officer Group Demonstrating Questionnaire Return Rate and Representativeness of Returnee Population Compared with the Total Population

\begin{tabular}{|c|c|c|c|c|c|}
\hline $\begin{array}{l}\begin{array}{l}\text { Demographic } \\
\text { Category }\end{array} \\
\text { TOTAL }\end{array}$ & \multicolumn{2}{|c|}{$\begin{array}{c}\text { Total } \\
\text { Population } \\
(\mathrm{N}=59)\end{array}$} & \multicolumn{2}{|c|}{$\begin{array}{c}\text { Returnee } \\
\text { Population } \\
(\mathrm{N}=41)\end{array}$} & $\begin{array}{c}\begin{array}{c}\text { Return Rate } \\
\text { From Total } \\
\text { Population }\end{array} \\
69 \% \\
\end{array}$ \\
\hline Males & (49) & $83 \%$ & (36) & $89 \%$ & $73 \%$ \\
\hline Females & (10) & $17 \%$ & (5) & $12 \%$ & $50 \%$ \\
\hline Pakeha & (37) & $63 \%$ & (26) & $63 \%$ & $70 \%$ \\
\hline Maori & (16) & $27 \%$ & (11) & $27 \%$ & $69 \%$ \\
\hline Pacific Island & (6) & $10 \%$ & (4) & $10 \%$ & $67 \%$ \\
\hline $0-4$ years service & (27) & $46 \%$ & (20) & $49 \%$ & $74 \%$ \\
\hline 4-10 years service & (23) & $39 \%$ & (14) & $34 \%$ & $61 \%$ \\
\hline 10+ years service & (9) & $15 \%$ & (7) & $17 \%$ & $78 \%$ \\
\hline
\end{tabular}

Note. Percentages rounded, totals may not equal $100 \%$.

The overall return rate of $69 \%$ was considered appropriate and acceptable for generalisation purposes for the given population and the 'postal' nature of the questionnaire as suggested by Moser and Kalton (1977). Return rates for specific demographic categories did show some variation. In particular, there were notably lower return rates for females and 4-10 years service personnel with a higher rate for those in the $10+$ years service category.

Informal discussion with non-returning females revealed a reluctance to respond on coded and hence identifiable paper given their already marginalised and precarious position within the jail. They were more comfortable with providing the data verbally, which was achieved through the course of the observation and interview phases. 
However, this information has not been included as a 'return' as it was not recorded on a questionnaire. This gender specific bias highlighted the importance of integrating multiple methods for the purposes of this project, along with the need to consider the data sets in combination where possible and not as distinct entities per se.

The reason for the different return rates within service length categories was less apparent. Most officers within the 0-4 years service category showed a perfunctory attitude towards compliance with such tasks. Officers within the 10+ years service category, which displayed a particularly high return rate, reported that they wanted to ensure that the senior voice was heard, expressing the view that interest in their occupational group was long overdue. Officers in the 4-10 years service category experienced difficulty expressing a rationale for their group's participation levels in the questionnaire stage of the research. This might have been because they were the most affected by service changes in the 1990s (see chapter three) and this then prompted levels of suspicion and distrust not present in the other groups of officers. However, over time as social access was achieved with them, the numbers of returns increased. Table 2.3 demonstrates that the returnee population was reasonably comparable to the total population set for Beta Jail.

Table 2.3

Demographic Details for the Beta Jail Prison Officer Group Demonstrating Questionnaire Return Rate and Representativeness of Returnee Population Compared with the Total Population

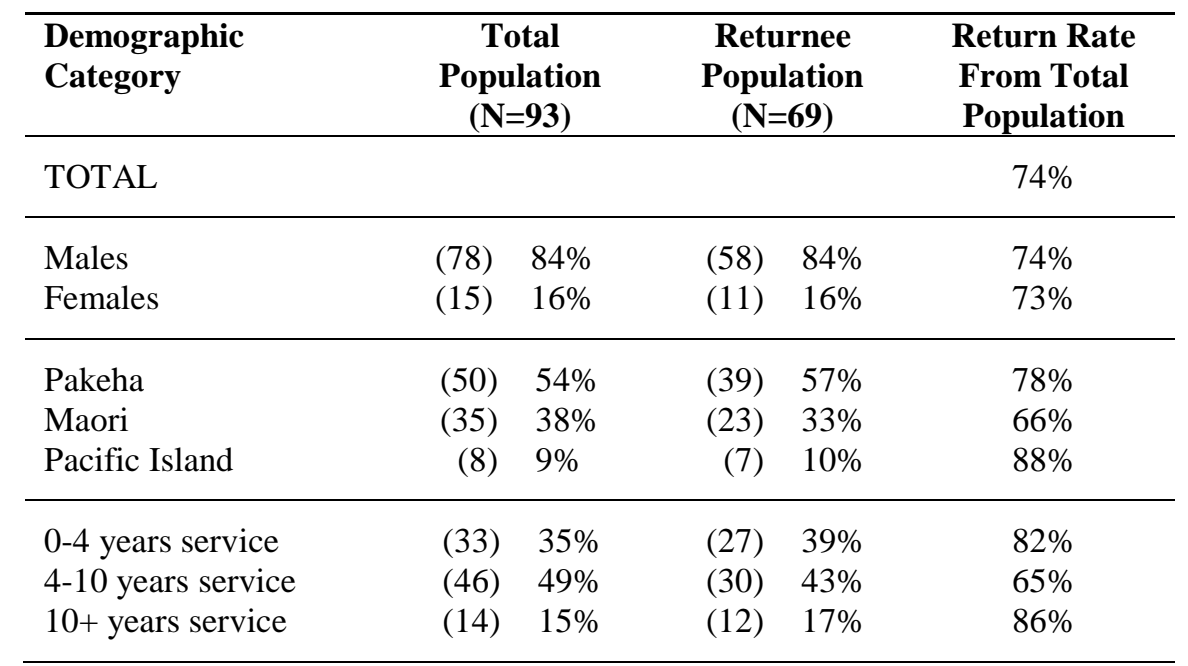

Note. Percentages rounded, totals may not equal 100\%. 
There were, however, a few notable divergences between the Beta Jail data set and the Alpha Jail data set. In the first instance, the rates of return for the 0-4 years service and $10+$ years service categories were exceptionally high. I would suggest that both these higher return rates and Beta Jail's elevated overall return rate of $74 \%$ were the result of two inter-related factors. The timing of Beta Jail's formal inclusion in the project allowed for greater social access to have developed prior to research proper. Similarly, the nature of Beta Jail units was conducive to an even deeper level of social access than that experienced within Alpha Jail.

Another important difference was that Beta Jail females had a comparable return rate with Beta Jail males as opposed to the difference reflected in the Alpha Jail figures. It may be that this difference reflected a higher level of acceptance of women within Beta Jail compared to Alpha Jail and hence a higher degree of confidence from the women to participate in this manner. This may be because Beta Jail had a lower security classification than Alpha Jail. Due to the different security classifications it was thought that higher security status related to more danger and threat and hence represented the more stereotypical image of a prison and requirements for being a prison officer in such a setting. In this way, females in the lower security classification units perceived they experienced less resistance or negativity to their presence as employees. This is turn translated into less apprehension from these women to commit comment to paper.

With respect to ethnic categories, there were two notable divergences. Maori displayed a lower return rate and Pacific Island people a higher return rate. A further analysis of the Maori data set revealed the overall Maori return rate was affected by the low return rate of one specific unit with a disproportionately high Maori staff level. Removing this unit from the data set results in a 78\% return rate for Maori, which was a more representative figure. During the research period there were a number of internal investigations of staff misconduct instigated by the Department of Corrections. The low response unit represented the most affected staff group, which may account for their reluctance to participate ${ }^{33}$.

\footnotetext{
${ }^{33}$ This effect similarly transferred to the observation phase where I was not convinced of the authenticity of the behaviours displayed within this unit. Regardless, staff generally displayed less
} 
The high rate of return from Pacific Island participants was a more difficult phenomenon to explain. It may be, however, that it reflected a cultural difference, whereby the value of 'loyalty' is strong for Pacific Island peoples. Once positive social relationships have developed, their desire to assist that person is stronger than other New Zealand ethnic groups. Therefore, once social access had been established with these participants, they likely responded due to a sense of social obligation.

Table 2.4 represents the combined population of Alpha Jail and Beta Jail. Overall, the returnee population showed reasonable comparability with the general population. The aforementioned trends continued through to the combined data with females, Maori and 4-10 years service length showing a lower return rate while Pacific Island, 4-10 years service and 10+ years service demonstrated higher rates.

\section{Table 2.4}

Demographic Details for the Complete Target Institution Prison Officer Group Demonstrating Questionnaire Return Rate and Representativeness of Returnee Population Compared with the Total Population

\begin{tabular}{|c|c|c|c|c|c|}
\hline \multirow{2}{*}{$\begin{array}{l}\text { Demographic } \\
\text { Category } \\
\text { TOTAL }\end{array}$} & \multicolumn{2}{|c|}{$\begin{array}{c}\text { Total } \\
\text { Population } \\
(\mathbf{N}=152)\end{array}$} & \multicolumn{2}{|c|}{$\begin{array}{c}\text { Returnee } \\
\text { Population } \\
(\mathbf{N}=110)\end{array}$} & \multirow{2}{*}{$\begin{array}{c}\text { Return Rate } \\
\text { From Total } \\
\text { Population }\end{array}$} \\
\hline & & & & & \\
\hline Males & (127) & $84 \%$ & (94) & $85 \%$ & $74 \%$ \\
\hline Females & (25) & $16 \%$ & (16) & $15 \%$ & $64 \%$ \\
\hline Pakeha & (87) & $57 \%$ & (65) & $59 \%$ & $75 \%$ \\
\hline Maori & (51) & $34 \%$ & (34) & $31 \%$ & $67 \%$ \\
\hline Pacific Island & (14) & $9 \%$ & (11) & $10 \%$ & $79 \%$ \\
\hline 0-4 years service & (60) & $39 \%$ & (47) & $43 \%$ & $78 \%$ \\
\hline 4-10 years service & (69) & $45 \%$ & (44) & $40 \%$ & $64 \%$ \\
\hline $10+$ years service & (23) & $15 \%$ & (19) & $17 \%$ & $83 \%$ \\
\hline
\end{tabular}

Note. Percentages rounded, totals may not equal $100 \%$. 


\section{The Observation Phase}

My observational research brought to light the subtlety of the interaction and symbolic interaction between prison officers and between officers and others. Such information could be lost or distorted using other methods. As Whyte (1984) reported of his classic Street Corner Society, when he directly questioned members of the Norton Street gang as to who their leader was, he was invariably told: "We have no leader. We are all equal” (p. 22). However, close association through participation told him otherwise. Similarly, when I questioned officers during interviews about such issues as informal hierarchies, their existence was often denied, but observation indicated the contrary. Such conflict between words and actions, however, was unlikely to be attempts by the actors to distort or misrepresent social arrangements. Rather, it was more likely a reflection of the taken for granted internalisation of facets of their social world.

However, observation alone would be insufficient to understand the dynamics of prison officer life. All behaviours have the potential for multiple understandings and clarification from the actor was necessary to supplement imposed meaning by the observer. As Weber (1962) demonstrated in his ideal type construction of the characteristics of social conduct, there can be no normatively correct or true meaning if it does not incorporate that which is imputed by the individuals involved (see also Eldridge, 1971; Runciman, 1978). As an example within the New Zealand prison context, it was observed that inmates walked on the right-hand side, against the social convention of New Zealand to stay to the left. Officers asserted this was the inmates' means of being 'anti-establishment'. Alternatively, officers tended to walk on the lefthand side. Now this could be interpreted as reflecting a pro-establishment position. However, discussion with officers revealed it was a safety issue whereby they felt uncomfortable with inmates following behind whereas from the other side there was full view. This disclosure in interview led to a vastly different understanding than simple observation would have provided. Hence observation and interviews provided the substantive data sources for this research. 
It should also be acknowledged that within the context of the prison, the inclusion of observation within the methodological design had additional functions beyond the direct data collection. Officers favourably viewed the time I spent at the prison during this phase, coupled with the additional time spent socially with participants. Such behaviour mirrored their occupational culture and values (as shall be discussed further in this thesis). O’Connor (1976) similarly alluded to this as a supporting technique of prison research as it demonstrated that the researcher was neither anxious in achieving their research objectives nor overly concerned with the prison per se, but, rather, was interested in the officers themselves. Thus, taking the time to be interested in everyday nuances of life, including interest in officers as people rather than as objects of research, encouraged more natural behaviour and willingness to participate in all facets of the research design. This was demonstrated by: the high return rate of questionnaires; the overwhelmingly high levels of cooperation and support from a traditionally closed occupational group during the observation phase; and the depth and detail of data offered in the interview phase which more than doubled the expected interview duration.

Being covert or overt during the observation phase is an important decision as it has many implications. It became evident that previous research on prison officers, their work and culture had been predominantly interview based. Where observation had been included it had generally been conducted covertly and/ or by prison employees past or present (see, variously, Kauffman, 1985 as an example of a past prison officer as researcher; Lombardo, 1981 as an example of a researcher and concomitant generalised prison employee; Marquart, 1983 as an example of a researcher adopting the job and role of a prison officer for covert research purposes). O’Connor (1976) compared problems of acceptance of an overt versus a covert observer and concluded that the issues were extremely similar. Indeed, he suggested that acceptance was more readily extended and avoided many of the issues of face management when the truth was told, particularly with institutional staff. Further, he argued that being overt permitted the researcher to concentrate their efforts on research without expelling copious concern and energy protecting their covert identity. As a white middle class female I perceived acceptance, whether covert or overt, as the primary obstacle to the success of the research. Therefore, overt observation was selected to ensure maximum concentration on research objectives. 


\section{Obtaining Informed Participation}

As observation of prison officers during their daily routines necessarily included their interactions with others in the prison community, it was necessary to consider informed participation from four other distinct groups in addition to the prison officers: management; non-prison officer staff; inmates; and visitors to the jail. This was initially achieved through the strategic display of posters and information sheets throughout the institution as previously outlined.

Managers were afforded full information, verbal and written, with the opportunity to question me during the initial introduction meeting initiated by upper management during the formal access phase. As full support for the project was proffered, and managers were aware they could inform me not to record details during periods they wished to be excluded, it was reasonable to assume informed consent had been obtained from this group. No manager requested I leave a situation or refrain from recording details of interactions for which they were involved.

I was invited to, and attended, a variety of non-prison officer staff meetings during the first weeks of the familiarisation phase. The project and its implications for involvement of non-officer staff was outlined at these meetings and questions raised were answered. In addition, ancillary staff who did not have scheduled meetings during this period were approached individually to discuss their potential involvement in the research. As with the managers, support and interest was offered. Similarly, no non-prison officer staff member requested exclusion of his or her interactions from data collection. Attempts were initiated to gain written consent from these actors. However, the high staff turnover rate of this group (due to resignations, secondments and so on) required abandonment of this objective. The assumption of information transfer between staff and via the posters and information sheets was necessary.

Immediately prior to entry of a unit, inmate specific information sheets were distributed under cell doors during night watch. Discussing the research with each prisoner was not possible within the given routines of the institution; therefore, informal information transfer was directed at key inmates who, I was confident, would subsequently disseminate it. As inmates moved through the institution, the 
requirement to inform in this manner lessened as brief discussion with inmates revealed a high level of understanding was filtering through informal channels. Given the highly transient nature of inmates, no formal/ written procedure for inclusion was sought. Notwithstanding this, information regarding how to exclude oneself or any particular interaction was clearly transmitted by me and remained my responsibility. No inmate withdrew participation in the project.

Prison visitors were more problematic. Not only were they highly transient, and therefore unlikely to have been exposed to information on multiple occasions as other actors within the community, there was also no adequate means to ensure awareness. Regardless, prison visitors represented the least active additional actor roles. On the few occasions where a prison visitor's presence was significant to the observation context, such as during the observation of District Prison Board hearings, informed consent was sought prior and formal introductions made at the time. No prison visitor requested exclusion.

As prison officers were the targeted participants of the research, more formalised systems were employed to ensure informed participation. During the first week of familiarisation at the institution I presented the details of the research, with particular emphasis on the observation phase, at staff meetings of each of the three Alpha Jail units. At this time, two officer staff meetings in Beta Jail were also attended with a presentation of the project as requested by the respective Unit Managers. Questions were answered and officers were assured individual consent would be obtained at the time of, and prior to, formal observation on shift. As informal channels of information flow preceded entry to Beta Jail units, presentation of the project occurred during the familiarisation period rather than prior, as was the case for Alpha Jail.

In one Alpha Jail unit staff meeting presentation of the research, staff collectively displayed concern and dissent arose as to whether as a group they were willing to facilitate my presence in their unit (they were concerned about tensions that existed between rival gangs in the unit at that time which they felt could be exacerbated by the introduction of a young non-officer female). By the end of the meeting access was successfully negotiated for the one week familiarisation period, at the end of which the unit staff could decide whether the effect of my presence was too significant to 
accommodate. A staff meeting at the end of this familiarisation week provided the sought access and support. Being accepted by this unit's staff after its concerns proved significant for the social access process: I had 'proved' myself 'capable' under trying circumstances.

\section{The Observation Process}

The process of the familiarisation phase of observation and formal observation differed only in regards to the latter constituting the data collection phase. For the familiarisation phase for any given unit, a purposive sample of five varied shifts were attended. At the start of any of these given shifts I approached the first available rostered officer and requested I spend the shift with them. Formal consent was not sought as data collection did not occur. No officer declined, though some preferred in the initial stages to split my time between multiple officers. As this facilitated greater exposure, did not affect the design in any substantive manner and effectively reduced the anxiety of officers, I complied with this preference.

The process of the formal observation phase was that for any one shift period one officer was designated the target officer under formal observation and any other actors, including officers, were included as informal participants of observation. For simplicity at the time of data collection, informally observed officers' details were recorded with the intention of removing the specific details if formal request to observe was subsequently declined. While two officers declined formal inclusion, none objected to informal collection - hence the recorded data remained intact. Selection of the target prison officer and roster of shifts attended is explained further in the following sampling frame section.

At the start of a designated formal observation shift the target officer was approached, supplied with an information sheet and two copies of the consent form and the research discussed. Data collection began upon verbal agreement to participate. A signed consent form for each shift was obtained from the target officer, though many preferred to complete this at the termination of the shift. All officers approached participated and none withdrew participation either in part or full. 
This process was repeated through the observation phase until the majority of officers and shift types had been recorded. The breakdown of shifts attended is shown in Table 2.5 .

Table 2.5

Summary Breakdown of Shifts by Jail and Type

\begin{tabular}{lccc}
\hline Shift Type & Alpha Jail & Beta Jail & TOTAL \\
\hline Familiarisation Shifts & 15 & 20 & $\mathbf{3 5}$ \\
Observation Shifts & 61 & 82 & $\mathbf{1 4 3}$ \\
TOTAL & $\mathbf{7 6}$ & $\mathbf{1 0 2}$ & $\mathbf{1 7 8}$ \\
\hline
\end{tabular}

During observation of the formal front stage, my style of research was formally semiparticipant observer. This involved the participant as observer and non-participant roles (see Morison, 1989). This required detailed consideration and balancing of data collection and participatory behaviour activities. Presentation of self was carefully negotiated to encompass this dual role. Civilian clothing of a similar style and colour as the officers' attire marked my affiliation with them. However, I did not carry keys or a radio. While I accompanied my target officer during all shift activities, my participation was at a superficial level only. Thus, official duties, such as muster checks, remained the task and responsibility of the officer. However, when inappropriate to do otherwise, I participated in the given activity. For example, if an officer was motivating a group of inmates by helping them dig a ditch, it would have been deleterious to my relationship with the target officer, potentially the officer group and certainly the dynamics of the given situation, not to similarly pick up a spade. For the majority of the time though, participation was passive and the observer role always took precedence.

Data was recorded in abbreviated note form in a hardback book carried throughout shift. Notes were recorded on a continuous time sampling frame whereby all events and interactions, no matter how seemingly trivial, were recorded for the entire duration of the shift. This was necessary to capture the quality, nature and frequency of ordinary behaviour and symbolism that would have been lost if the context were 
removed via interval type sampling or use of a predetermined schedule. Where required, additional notes were added after the shift.

According to Martin (2000), an unstated protocol for field researchers is not to be seen to be writing anything down, particularly while observing sensitive topics. While there is merit in this proposition, it is my view that one's memory does not have the recall capacity to subsequently record the specifics of serial non-significant events over the course of eight hours with any claim to accuracy. The effects of such features of memory as primacy or saliency of events would necessarily have rendered the data suspect in the given theoretical frame. Therefore, overt and continuous writing was employed. While this initially provided a source of amusement, officers quickly became accustomed to the continuous data recording. An added benefit of this style was that my attention to any given situation did not noticeably vary and hence alert officers to possible sources of 'interest'.

The following is an example of a five minute segment of notes extracted from a notebook:

[PO\#1] leaves [guardroom] to go to tea break. [PO\#2] enters [guard-room] and checks who is on
lock in the book then leaves quietly. [PO\#1] back in [guardroom] checking muster book for how
long a lock [inmate] has been there (to see if he's to continue on lock). Nurse is in background
trying to organise medical but no one is listening to her directly. [PO\#1] leaves telling [PO\#3]
'Can't find [inmates surname]'. [PO\#3] asks [PO\#4] to help the nurse by knocking on the [guard-
room] door and pointing at her. [PO\#5] walks into [guard-room] at this moment and says he'll do it.
[PO\#4] shrugs.
$\mathbf{1 7 5 9}$
[PO\#3] heads up [wing name] unlocking side to side. Half way up an inmate calls out to [PO\#3]
'Hey you missed one'. [PO\#3] calls out 'Back in a minute'. Finishes wing unlock. Walks back up
wing. Stops at the cell on the way and tells the inmate 'you're on lock'. The inmate disputes 'no
way Mr.' and pulls face. [PO\#3] looks up at date on card above door and unlocks. [PO\#3] walks
back up wing staring at ground.
$\mathbf{1 8 0 2}$
[PO\#3] returns to [guard-room] and says frustrated to [PO\#4] 'I don't know who should be more
afraid, it's these things that show up on the probation report and it will on his'. [PO\#4] looks up at
[PO\#3] but says nothing.
[PO\#3\&4] listen to nurse [outside door] chatting with an inmate about medication for stress (inmate
under review for going on observation).

Figure 2.1 Example of extract from field journal. 
In addition to the formal observation notes, personal diary entries were recorded daily in a separate set of journals. This process was vital not only for the validity of the research, but also for my own well-being. It provided a means of detaching myself from the environment. A form of cathartic purging, as it were, which allowed the emotions of the day-both positive and negative-to be expressed on paper. Professionally, these notes aided my reflections on the research process, allowing me to evaluate the effects I may have had on the research process and its outcomes. It enforced an objective analysis of the self and changes in perspective that might have affected the interactions occurring at the time as well as subsequent analysis and theory development. Similarly, it provided a cross reference to measure and assess the quality, nature and effect of 'going native' (or identification with the participant group) and how this may have impacted on the research. Liebling (1999) proposed that changes in one's self, as identified through journal entries, was data in itself as it provided insight into the things that were distinctive about the people that one was studying.

Data analysis of the field notebooks involved two distinct phases. In the first instance, these were coded thematically to elucidate areas of inquiry for the generalised interview. The second phase of observation data analysis included and took place alongside the interpretative analysis of the interview data.

\section{Sampling frame.}

The sampling frame for Alpha and Beta Jails varied. For Alpha Jail, the rostered staff of all three units were included within the population's sampling frame. Selection was initially based on random assignment to the designated shift roster ${ }^{34}$. Towards the end of each week I developed a unit specific research schedule for the following week that facilitated coverage of each shift type by the end of the observation phase and was realistically achievable in terms of worked hours by myself. Seven days a week were covered with a minimum of seven eight hour shifts each week. This research schedule was not supplied to the prison or unit staff for two reasons. In the first instance, it

\footnotetext{
${ }^{34}$ All units had a unit specific standardised cyclical roster. Each unit's roster varied in its pattern along designated routes to encompass the differential tasks required for the functioning of the given unit and formal absenteeism of staff. Position and assignment to the roster was predetermined and hence location was random for the purposes of this research.
} 
remained confidential to remove the potential for any untoward movement of staff within the roster. Secondly, there would be no formal record as to my times and movement within the prison and hence data could not be linked to individual staff members.

The majority of the designated shifts had a potential sampling population of two to three officers. In the first instance, criterion sampling was used whereby whichever officer had not previously been formally observed was selected. Where multiple officers matched this criterion accidental sampling was used whereby the first of the potential participants to arrive on shift was selected. Alternatively, if it was known that one of the potential criterion participants would soon be removed from the population sample due to annual leave, secondment and so on, this person was given preference and purposive sampling was used.

Where possible, all shift types and all officers were sampled. This, however, did not represent the entire population of prison officers because of leave entitlements, secondments and the like. Some officers were observed more than once to incorporate all shift types and some shift types were included more than once to maximise officer inclusion. During any given shift period, multiple officers were observed at various intervals in addition to the target officer. Therefore multiple task/ shift type variations for each officer was possible, albeit limited.

Beta Jail sampling frame did not vary in respect to target officer selection. However, the units included within the sampling population were limited. Two of the six Beta Jail units were excluded from the formal observation phase ${ }^{35}$. The reason for this was as follows. One of the excluded units opened for operation shortly after my research commenced at the target prison. For some months it experienced difficulty establishing routinised performance as evidenced by several multiple-inmate disturbances. After these, the unit was formally closed for maintenance and the staff relocated throughout the prison. The period in which I was scheduled to conduct the observation phase coincided with the re-opening of the unit. While it would have been

\footnotetext{
${ }^{35}$ All officers from the two excluded units were included in the questionnaire and interview sampling populations. Many of these officers were formally and informally observed while seconded to, or were at the time rostered members of, other units. For this reason, their demographic details have been incorporated within the sampling population shown in Table 2.7.
} 
interesting to observe the re-establishment of the unit, my supervisor and I deemed it inappropriate. In the first instance, it could not be ethically justified for the purposes of my research to potentially affect the developing group dynamics of the officer and inmate groups, particularly as I was aware of the social and political issues surrounding the situation. Second, as it did not represent the ordinary everyday experience of prison officer work, it was beyond the scope of my study.

The second unit was excluded for similar reasons. It was opened towards the end of the observation phase. Further, it was designed as a specialist unit with a highly concentrated inmate programme and multiple psychological staff alongside the officers. It, too, represented the non-typical.

All officers approached for inclusion in the observation phase participated. Between both Alpha and Beta Jails, only two prison officers requested not to be included in the sampling population. Both requests were made prior to formal approach for inclusion. One of the two then offered to be observed while I was assigned to another officer. The second officer supported the research, but did not like the idea of being 'watched'.

\section{Participation rate.}

Table 2.6 demonstrates that $80 \%$ of the Alpha Jail population were formally observed and that the observed population was representative of the total population. Of the twelve officers who did not participate, one was absent on secondment, two elected not to be included, and nine were transfers in and out of Alpha Jail during the designated period. 


\section{Table 2.6}

Demographic Details for the Alpha Jail Prison Officer Group Demonstrating Representativeness of the Observed Population Compared with the Total Population

\begin{tabular}{|c|c|c|c|c|}
\hline $\begin{array}{l}\text { Demographic } \\
\text { Category }\end{array}$ & \multicolumn{2}{|c|}{$\begin{array}{l}\text { Total Population } \\
\text { (N=59) }\end{array}$} & \multicolumn{2}{|c|}{$\begin{array}{c}\text { Observed } \\
\text { Population } \\
(\mathrm{N}=47)\end{array}$} \\
\hline TOTAL & & & \multicolumn{2}{|c|}{$80 \%$} \\
\hline Males & (49) & $83 \%$ & (39) & $83 \%$ \\
\hline Females & (10) & $17 \%$ & (8) & $17 \%$ \\
\hline Pakeha & (39) & $66 \%$ & (29) & $62 \%$ \\
\hline Maori & (15) & $25 \%$ & (14) & $30 \%$ \\
\hline Pacific Island & (5) & $8 \%$ & (4) & $9 \%$ \\
\hline 0-4 years service & (28) & $47 \%$ & (22) & $47 \%$ \\
\hline 4-10 years service & (22) & $37 \%$ & (18) & $38 \%$ \\
\hline 10+ years service & (9) & $15 \%$ & (7) & $15 \%$ \\
\hline
\end{tabular}

Note. Percentages rounded, totals may not equal 100\%.

Table 2.7 demonstrates an overall observation rate for Beta Jail of 70\%. This figure, however, included fourteen officers unable to be included due to movements associated with the two exclusion units. Removing these officers provided a comparable observation rate with Alpha Jail of 81\%. Of the thirty officers who did not participate, fourteen were 'non-unit' associated, six were on annual leave, nine transferred in or out of the Beta Jail during the designated period, and one additional transferred officer had elected not to be included while in Alpha Jail and was thus not included whilst in Beta Jail. Overall the observed population was reasonably comparable to the total population. 
Each Day is Different: Prison Officers and Their Work

Table 2.7

Demographic Details for the Beta Jail Prison Officer Group Demonstrating Representativeness of the Observed Population Compared with the Total Population

\begin{tabular}{|c|c|c|c|c|}
\hline $\begin{array}{l}\text { Demographic } \\
\text { Category }\end{array}$ & \multicolumn{2}{|c|}{$\begin{array}{c}\text { Total Population } \\
(\mathrm{N}=99)\end{array}$} & \multicolumn{2}{|c|}{$\begin{array}{c}\text { Observed } \\
\text { Population }(\mathrm{N}=69)\end{array}$} \\
\hline TOTAL & & & \multicolumn{2}{|c|}{$70 \%$} \\
\hline Males & (81) & $82 \%$ & (59) & $86 \%$ \\
\hline Females & (18) & $18 \%$ & (10) & $14 \%$ \\
\hline Pakeha & (52) & $53 \%$ & (36) & $52 \%$ \\
\hline Maori & (38) & $38 \%$ & (26) & $38 \%$ \\
\hline Pacific Island & (9) & $9 \%$ & (7) & $10 \%$ \\
\hline 0-4 years service & (34) & $34 \%$ & (27) & $39 \%$ \\
\hline 4-10 years service & (51) & $52 \%$ & (34) & $49 \%$ \\
\hline $10+$ years service & (14) & $14 \%$ & (8) & $12 \%$ \\
\hline
\end{tabular}

Note. Percentages rounded, totals may not equal $100 \%$.

Table 2.8 demonstrates that across the institution a high level of representativeness was achieved in the observed population set. The observation rate of $73 \%$ increased to $81 \%$ when the fourteen 'non-unit' staff members were removed.

Table 2.8

Demographic Details for the Complete Target Institution Prison Officer Group Demonstrating Representativeness of the Observed Population Compared with the Total Population

\begin{tabular}{|c|c|c|c|c|}
\hline $\begin{array}{l}\text { Demographic } \\
\text { Category } \\
\end{array}$ & \multicolumn{2}{|c|}{$\begin{array}{c}\text { Total Population } \\
(\mathrm{N}=158)\end{array}$} & \multicolumn{2}{|c|}{$\begin{array}{c}\text { Observed } \\
\text { Population }(\mathrm{N}=116)\end{array}$} \\
\hline TOTAL & & & \multicolumn{2}{|c|}{$73 \%$} \\
\hline Males & (130) & $82 \%$ & (98) & $84 \%$ \\
\hline Females & $(28)$ & $18 \%$ & (18) & $16 \%$ \\
\hline Pakeha & (91) & $58 \%$ & (65) & $56 \%$ \\
\hline Maori & (53) & $34 \%$ & $(40)$ & $34 \%$ \\
\hline Pacific Island & (14) & $9 \%$ & (11) & $9 \%$ \\
\hline 0-4 years service & (62) & $39 \%$ & (49) & $42 \%$ \\
\hline 4-10 years service & (73) & $46 \%$ & (52) & $45 \%$ \\
\hline 10+ years service & (23) & $15 \%$ & (15) & $13 \%$ \\
\hline
\end{tabular}

Note. Percentages rounded, totals may not equal $100 \%$. 


\section{The Interviews}

The 'specialised' interviews occurred within two weeks of a specified serious incident. One third occurred after serious assaults on individual officers. The rest followed a riot that occurred in Green Unit during the course of my research. The series of events leading to the riot involved six inmates. They were unhappy with the proposed reduction in unlock time as a Departmental response to low staff numbers and became intoxicated (drinking prison style homebrew) while unsupervised in the kitchen. Upon discovery, officers attempted to lock the inmates down in their cells. A fracas developed in the wing which led to an officer being seriously assaulted. Several other inmates joined in the activity and weapons were produced - prompting the immediate withdrawal of the officers. The entire prison was put on lockdown to free other unit officers to assist. An impromptu telephone chain (between officers) brought off-duty officers and officers from throughout the region in to help. Police (Armed Offenders Squad) and fire services were called by management to assist. It took around eight hours to regain control of the wing. Inmates had destroyed property and set fires, making the entire wing uninhabitable for some time.

The inclusion of these interviews arose spontaneously in response to these events and requests from participants, rather than being part of the original research design. It was my judgement that any predetermined structure would impose topic relevance and potentially hinder the given individual's recovery, which could not be ethically justified for the purposes of my research. Therefore, these interviews were deliberately unstructured and non-directive. A major benefit of this approach was that it afforded the greatest direction from interviewees and hence permitted the saliency of events or effects to emerge naturally.

The data from these specialised interviews was useful both as a reflection of the actor's experience of such incidents at the time of occurrence, and as a comparison with views projected after a time lapse from the said events as provided in the 'generalised' interviews or informal discussions during the observation phase. Of particular interest was the spontaneous use of accounts by the actors and subsequent change or development of such explanations during the remaining observation phase 
and later generalised interviews. For example, these interviews demonstrated the progression of the particular incident from the basic 'immediate details', to highly complex 'war stories' that perpetuated certain goals with the selective removal of particular details or addition of others.

In contrast, the 'generalised' interviews were included in the research design to provide in-depth information to contextualise and expand on issues raised from the questionnaires and observation phase (for a copy of the interview guide see Appendix C). Interviews were used in combination with informal discussion during the observation phase as this afforded participants an opportunity to raise or expand on topics in a more private arena.

The semi-structured nature of the generalised interviews permitted a more informal and relaxed approach which was conducive to the informal relationships I had established. Indeed, Liebling (1999) argued that more formalised structure in the interview process had the potential to deleteriously affect data collection as participants expect a certain level of 'understanding' from the researcher as portrayed in the question format when prior knowledge exists. Further, the 'relaxed' approach lessened inhibitions and allowed the introduction of sensitive topics in a nonthreatening manner with a perception of control and direction by the interviewee, thereby heightening the level and depth of disclosure.

\section{The Specialised Interview Process}

Specialised interviews occurred within two weeks of the specific incident. Interviews occurred at the prison either during or immediately following the interviewee's designated shift. Both the time and location were at the discretion of the interviewee and availability of space. With one exception ${ }^{36}$, interviews were conducted privately, albeit with the occasional brief disruption, during which time the interview ceased and resumed at the end of the interruption.

\footnotetext{
${ }^{36}$ One interviewee requested the presence of another prison officer.
} 
Prior to each interview commencing, interviewees were provided with an information sheet and two copies of the interview consent form, one form for their records and one to be completed prior to the interview proper. All interviewees agreed to cassette recording. Interview duration varied from one to three hours with an average of around two recorded hours. General issue inclusion was at the officer's discretion; however, the primary focus remained on the specific incident.

Cassette tapes were transcribed onto computer disk files. Identifying details, such as names, were replaced by codes. Cassette tapes were destroyed following transcription. Hard copy transcripts were coded thematically to aid analysis.

\section{Sampling frame.}

Purposive or targeted interviewee selection was not considered appropriate or ethically justifiable. I did not wish to cause unnecessary stress and raise emotions during an already problematic time for the given individuals. Therefore, while not ideal, self-selection and snowball were the techniques used to generate these interviews. Indeed, self-selected interviewees directly requested and hence initiated the inclusion of this segment of the methodology. Interviews with these participants were scheduled at their earliest convenience. Self-selection interviewees, along with other officers, volunteered names of further officers to approach for inclusion. No officers approached for interview declined. The specialised interview period was limited to two weeks following specific events, restricting the achievable number of data sets.

\section{Participation rate.}

As Table 2.9 demonstrates, the specialised interviewee population was considerably skewed. This was unavoidable given the sampling frame employed, nor did time constraints afford a remedy period in which to conduct more interviews. Regardless, caution was employed in analysis and hesitation employed with respect to the limitations of generalisation. Notwithstanding this, the data did provide some indication of immediate reactions to such events. Further, considerable similarity of opinion was derived from informal discussion with other officers of varying 
demographic categories during the observation phase, lending support to the validity of the data gathered during these interviews.

Table 2.9

Demographic Details of the Specialised Interviewees

\begin{tabular}{lc}
\hline \multicolumn{1}{c}{$\begin{array}{c}\text { Demographic } \\
\text { Category }\end{array}$} & $\begin{array}{c}\text { Specialised } \\
\text { Interviewees } \\
(\mathbf{N}=\mathbf{8})\end{array}$ \\
\hline Males & 7 \\
Females & 1 \\
\hline Pakeha & 7 \\
Maori & 1 \\
Pacific Island & 0 \\
\hline 0-4 years service & 2 \\
4-10 years service & 5 \\
10+ years service & 1 \\
\hline
\end{tabular}

\section{The Generalised Interview Process}

The generalised interview process commenced after a brief data analysis period at the termination of the observation phase of Beta Jail. Interviewees were provided the option of the interview occurring in their own time and preferred location or at a convenient time during shift at a private location of their choice at the prison ${ }^{37}$. For those participants who chose to be interviewed during shift hours, night watch provided the most suitable time period to minimise both interruptions to the interview and disruption to the unit and other staff. As unit rosters were cyclical in nature, this resulted in some time delays between interviews as I waited for the target officer to naturally progress to this phase of the roster. However, not all who selected the prison location were able to accommodate the interview during a night watch shift. For these individuals, the interview was typically conducted during or after an appropriate shift. In sum, of the thirty one interviews, twenty eight occurred at the prison, and three off site.

\footnotetext{
${ }^{37}$ As a recognition that this phase of the research potentially impinged on the officer's personal time, inclusion in one of three bar/ restaurant voucher draws was included in the design. The draw, rather than payment, was proffered as it was not a direct inducement to participate.
} 
As with the specialised interviews, prior to each interview commencing, interviewees were provided with an information sheet and two copies of the interview consent form, one form for their records and one to be completed prior to the interview proper. All interviewees agreed to cassette recording. On three occasions different interviewees requested a pause in recording to discuss or elaborate on issues they did not want cassette recorded. I do not believe that the recorded data set then became biased as a result: other interviewees and officers discussed the same topics, either during the observation phase or interview sessions. Interview duration varied from one and a half to four hours with an average of around two and a half recorded hours.

Interviews were semi-structured (for a copy of the interview schedule see Appendix C). All topic areas and questions were presented to each interviewee. Specific wording of questions varied only slightly between interviewees. The topic order varied considerably according to the flow and direction of the interviewee. While this complicated subsequent analysis, this flexible approach facilitated the quality and depth of the data. The cassette tapes were later transcribed onto computer disk files. Identifying details, such as names, were replaced by codes. Cassette tapes were destroyed following transcription. Hard copy transcripts were coded thematically to aid analysis.

Analysis of both the specialised and generalised interviews was dual in approach and involved reflection incorporating data from the questionnaire and observation phases. The first phase involved examining the transcripts to reveal the dominant or recurring narrative themes. The second phase moved beyond the thematic to interpretative.

\section{Sampling frame.}

Cluster purposive sampling was the technique employed for the generalised interviews. An initial list of potential interviewees was compiled from those who expressed interest in participation via the questionnaire consent form, observation consent form or verbalised interest directly to myself during the course of my research. Individuals were then divided according to their location in either Alpha Jail or Beta Jail at the time of interview selection. Alpha Jail and Beta Jail potential interviewee lists were then clustered according to the standardised demographic 
categories of gender, ethnicity and length of service. In total there were thirty six potential clustered population sets. Eleven of these categories did not have members thereby leaving twenty five clustered population sets from which to sample ${ }^{38}$. Potential interviewees who would not be present during the first month of the interview process were noted for exclusion from the initial selection phase ${ }^{39}$.

For the initial selection phase, fifteen interviewees were selected from both Alpha Jail and Beta Jail totalling thirty interviewees. Interviewees were selected purposively in that attempts were made to select a member of each available population set. Where a population set had multiple members, the participant was randomly selected. The population set of intended interviewees was skewed in favour of minority groups to ensure reasonable representation of each demographic category. After the initial selection process an additional officer requested inclusion. It was deemed acceptable to incorporate an interview with this individual into the data set as it did not affect representativeness of the sample. Hence the number of interviews was thirty one. This interviewee’s demographic details have been incorporated within the Beta Jail figures to maintain confidentiality.

Initial selection officers were contacted in person or by phone to initiate the interview process. All initial selection officers agreed to participate and so a secondary selection process was not required. Tables 2.10 through 2.12 illustrate the actual interviewee population sets representativeness relative to the potential interviewee population sets.

\footnotetext{
${ }^{38}$ To avoid repetition of figures, the population sets presence or absence can be easily derived from Tables 2.10 through 2.12 .

${ }^{39}$ To maintain confidentiality of these officers, unit rosters for each unit were reviewed and potential absentees were considered to be those potential interviewees on secondment to other institutions, on annual leave, extended sick leave and other unspecified leave without pay. The demographic details of these initially excluded participants were included in Tables 2.10 through 2.12 as they remained potential interviewees for a secondary selection phase.
} 
Table 2.10

Clustered Demographic Details for the Male, Female and Combined Gender Alpha Jail Prison Officer Potential Versus Actual Interviewee Population Sets

\begin{tabular}{|c|c|c|c|c|c|c|c|c|c|}
\hline & & \multicolumn{4}{|c|}{ ALPHA JAIL POTENTIAL } & \multicolumn{4}{|c|}{ ALPHA JAIL ACTUAL } \\
\hline & & $0-4$ years & 4-10 years & $10+$ years & TOTAL & $0-4$ years & 4-10 years & $10+$ years & TOTAL \\
\hline \multirow[t]{4}{*}{ MALES } & Pakeha & 16 & 9 & 3 & 28 & 2 & 2 & 1 & 5 \\
\hline & Maori & 3 & 2 & 3 & 8 & 2 & 1 & 0 & 3 \\
\hline & Pacific Island & 2 & 0 & 1 & 3 & 1 & 0 & 0 & 1 \\
\hline & TOTAL & 21 & 11 & 7 & 39 & 5 & 3 & 1 & 9 \\
\hline \multirow[t]{4}{*}{ FEMALES } & Pakeha & 2 & 3 & 0 & 5 & 2 & 1 & 0 & 3 \\
\hline & Maori & 0 & 3 & 0 & 3 & 0 & 2 & 0 & 2 \\
\hline & Pacific Island & 0 & 1 & 0 & 1 & 0 & 1 & 0 & 1 \\
\hline & TOTAL & 2 & 7 & $\mathbf{0}$ & 9 & 2 & 4 & $\mathbf{0}$ & 6 \\
\hline COMBINED & Pakeha & 18 & 12 & 3 & 33 & 4 & 3 & 1 & 8 \\
\hline \multirow[t]{3}{*}{ GENDERS } & Maori & 3 & 5 & 3 & 11 & 2 & 3 & 0 & 5 \\
\hline & Pacific Island & 2 & 1 & 1 & 4 & 1 & 1 & 0 & 2 \\
\hline & TOTAL & 23 & 18 & 7 & 48 & 7 & 7 & 1 & 15 \\
\hline
\end{tabular}


Table 2.11

Clustered Demographic Details for the Male, Female and Combined Gender Beta Jail Prison Officer Potential Versus Actual Interviewee Population Sets

\begin{tabular}{|c|c|c|c|c|c|c|c|c|c|}
\hline & & \multicolumn{4}{|c|}{ BETA JAIL POTENTIAL } & \multicolumn{4}{|c|}{ BETA JAIL ACTUAL } \\
\hline & & 0-4 years & 4-10 years & $10+$ years & TOTAL & $0-4$ years & 4-10 years & $10+$ years & TOTAL \\
\hline \multirow[t]{4}{*}{ MALES } & Pakeha & 16 & 12 & 5 & 33 & 3 & 4 & 2 & 9 \\
\hline & Maori & 5 & 13 & 5 & 23 & 0 & 1 & 0 & 1 \\
\hline & Pacific Island & 0 & 2 & 2 & 4 & 0 & 1 & 1 & 2 \\
\hline & TOTAL & 21 & 27 & 12 & 60 & 3 & 6 & 3 & 12 \\
\hline \multirow[t]{4}{*}{ FEMALES } & Pakeha & 3 & 3 & 0 & 6 & 1 & 1 & 0 & 2 \\
\hline & Maori & 1 & 3 & 0 & 4 & 1 & 1 & 0 & 2 \\
\hline & Pacific Island & 1 & 0 & 0 & 1 & 0 & 0 & 0 & $\mathbf{0}$ \\
\hline & TOTAL & 5 & 6 & $\mathbf{0}$ & 11 & 2 & 2 & $\mathbf{0}$ & 4 \\
\hline COMBINED & Pakeha & 19 & 15 & 5 & 39 & 4 & 5 & 2 & 11 \\
\hline \multirow[t]{3}{*}{ GENDERS } & Maori & 6 & 16 & 5 & 27 & 1 & 2 & 0 & 3 \\
\hline & Pacific Island & 1 & 2 & 2 & 5 & 0 & 1 & 1 & 2 \\
\hline & TOTAL & 26 & 33 & 12 & 71 & 5 & 8 & 3 & 16 \\
\hline
\end{tabular}


Table 2.12

Clustered Demographic Details for the Male, Female and Combined Gender Total Prison Prison Officer Potential Versus Actual Interviewee Population Sets

\begin{tabular}{|c|c|c|c|c|c|c|c|c|c|}
\hline & & \multicolumn{4}{|c|}{ TOTAL PRISON POTENTIAL } & \multicolumn{4}{|c|}{ TOTAL PRISON ACTUAL } \\
\hline & & $0-4$ years & 4-10 years & $10+$ years & TOTAL & 0-4 years & 4-10 years & $10+$ years & TOTAL \\
\hline \multirow[t]{4}{*}{ MALES } & Pakeha & 32 & 21 & 8 & 61 & 5 & 6 & 3 & 14 \\
\hline & Maori & 8 & 15 & 8 & 31 & 2 & 2 & 0 & 4 \\
\hline & Pacific Island & 2 & 2 & 3 & 7 & 1 & 1 & 1 & 3 \\
\hline & TOTAL & 42 & 38 & 19 & 99 & 8 & 9 & 4 & 21 \\
\hline \multirow[t]{4}{*}{ FEMALES } & Pakeha & 5 & 6 & 0 & 11 & 3 & 2 & 0 & 5 \\
\hline & Maori & 1 & 6 & 0 & 7 & 1 & 3 & 0 & 4 \\
\hline & Pacific Island & 1 & 1 & 0 & 2 & 0 & 1 & 0 & 1 \\
\hline & TOTAL & 7 & 13 & $\mathbf{0}$ & 20 & 4 & 6 & $\mathbf{0}$ & 10 \\
\hline COMBINED & Pakeha & 37 & 27 & 8 & 72 & 8 & 8 & 3 & 19 \\
\hline \multirow[t]{3}{*}{ GENDERS } & Maori & 9 & 21 & 8 & 38 & 3 & 5 & 0 & 8 \\
\hline & Pacific Island & 3 & 3 & 3 & 9 & 1 & 2 & 1 & 4 \\
\hline & TOTAL & 49 & 51 & 19 & 119 & 12 & 15 & 4 & 31 \\
\hline
\end{tabular}




\section{The Research In Practice}

Although I entered this research field with all the rigour and discipline expected of a social scientist, this proved to be a naïve approach and did not ensure a trouble free research experience. The most significant realisation upon entering the field was that textbook methodological principles and ethical practices were not directly transferable to real life experiences. The most difficult part of the research process came to be the necessary development of a rationalised response to this seemingly incongruous situation: all of which had to be performed while presenting a suitable presentation of self in the field so as not to jeopardise the research. Personal judgement, intuition and creative adaptive instinct prevented the failure of a purely scientific approach. This section will highlight some of the issues I encountered.

\section{Alternative Approach To Ethics}

It became evident very quickly that the traditional approach to social science research, with preconceived determinants for behaviour, was neither realistic nor viable within the context of the prison as a research site. To ensure success, an alternative approach to the ethics of social science research was required. Arguably, it should not surprise us that alternative approaches are particularly relevant for criminology. Dixon (1997) argued that by the very nature of criminology, we, as researchers, are liable to observe, even become indirectly involved in, civil and/ or legal violations - a set of occurrences that the traditional approach does not accommodate. Marquart (1986) referred to this situation as obtaining 'guilty knowledge' (see also Klockars \& O’Connor, 1979; Lee, 1993). It was how we react to this guilty knowledge, within the bounds of ethical considerations, which determined the fate of any given project. At times, however, this might require a more liberal interpretation of ethical behaviour within particular contexts or fields, especially within an institution such as the prison. Therefore, the ethical approach I adopted for the purposes of this research was actutilitarian or situational ethics. Fletcher (1978, p. 423) defined this approach as "a utilitarian or consequentialist ethics, motivated by concern for human well-being, 
decisionally flexible in method, and guided in its judgements by the greatest good realizable rather than by adhering to prefabricated norms or moral rules”.

Leo (1997) criticised such approaches as relativist and a form of 'intellectual laziness'. However, I would contend, as does Wellman (1978), that situational ethics does maintain ethical absolutism and is therefore closer to standard ethical practices than one may assume, albeit varying in presentation according to the field situation. That is, researchers do not change the principles of ethical behaviour, but may need to alter how those principles are represented within particular contexts. Further, as O’Connor (1976) highlighted, such frequent and varied analyses of ethical considerations actually placed greater pressures and strain upon the researcher than relying on the more formal or predetermined approaches and methods - hence the reference to 'laziness' is unjustified.

To demonstrate, consider the standardised human ethics committee requirement of obtaining informed consent. To fulfil these requirements numerous media, as per the rule-utilitarian approach, were employed ranging from posters and information sheets through to formal and informal meetings. However, it quickly became apparent that officers were less influenced by such measures than anticipated and viewed such techniques with suspicion and as mere bureaucratic rhetoric. Put simply, they were not interested in whether I stored the data in locked filing cabinets and so on. Rather, they wanted to know whether I was someone they wanted in their midst and privy to their experiences. Therefore, of greater value and importance to officers was my reaction to character tests initiated by both officers and inmates. This experience was not unique to myself and has been similarly presented as pivotal to the observational research enterprise by such authors as Marquart (1986), Martin (2000) and O’Connor (1976).

In this research, these character tests ranged from the casual observation of my actions in everyday situations, through to deliberate and manipulated sets of circumstances intended to challenge me morally, mentally and physically. Tests included such incidents as: my reaction to observing or knowing of violations of prison rules by 
officers and/ or inmates, progressing over time from very minor ${ }^{40}$ to serious ${ }^{41}$; my reaction to intentionally directed exposure to pornography ${ }^{42}$ and other offensive material and language; willingness to participate in various officer activities beyond the realm of the research proper ${ }^{43}$ and so on. Essentially, was I prepared to tolerate behaviour that was typically offensive to middle class mores; could I be trusted with guilty knowledge; and was I prepared to move outside of my usual boundaries by challenging my predetermined ethical constructs? It should be noted here that these tests and my reaction to them were not in breach of the formal contract between the prison and myself (for a copy see Appendix B).

At times I was challenged at both my personal and professional levels of ethics and morality. Notwithstanding this, absolutist principles were involved, hence my argument that it was not a relativist approach. In the process of attempting to pass the tests, and thereby demonstrating to officers that the research and I were credible, I would evaluate the officers' responses within the bounds of academic ethical constraints and behave according to principles that were acceptable to both whilst maintaining certain boundaries. Importantly, officers were not expecting unconditional acceptance of all tests or behaviours. Indeed, my refusal to accept or do something was equally part of the testing process. At times it was a difficult balance.

\footnotetext{
${ }^{40} \mathrm{I}$ am referring here to acts that constitute rule violations but were minimal in direct negative effect. For example, it was not uncommon for officers on evening/ night shifts to eat meals from the inmates' general rations. Indeed, inmates often prepared meals specifically for this purpose and officers kept them aside. During one of the familiarisation periods of a unit an officer handed me a plate of food and requested I eat. Upon doing so he announced triumphantly that now I could not report them as I had broken the rules too.

${ }^{41}$ Some of the serious incidents did involve direct negative effect and harm. Reaction to these required particular consideration, self-examination and reflection. Examples of such incidences include knowledge of performed or intended officer-to-inmate, inmate-to-inmate and even officer-to-officer violence. To consider, here is an example of a serious incident that I was directly involved in, albeit not on institution grounds. While attending an officers' social function, as a display of group ownership, an officer assaulted my accompanying friend leaving him with a fractured cheekbone. It was one direct hit, not a prolonged attack. Regardless, the police were called amongst the furore that resulted. The police officers seemingly had an indication of events as it was I that was rhetorically posed 'Nothing wrong here m’am?’. My friend had indicated that his wish was to leave and not become more involved, therefore, I withheld comment. In so doing, I was withholding information regarding a crime. Several officers made a point of commenting to me about the incident and my choice to remain silent. With the exception of just a few officers the theme was that it was the correct response to maintain group acceptance. One officer, albeit rather menacingly, announced to me that 'you continue to watch our back and we'll watch yours'.

${ }^{42}$ This incident was not only an attempt to affront my moral sensibilities but also to gauge my reaction to contraband material as pornography was not permitted within the prison.

${ }^{43}$ This involved a wide range of activities such as attending social functions and funerals through to baby-sitting an officer's children, folding laundry and attending midnight call-outs for transport essentially the variety of tasks that 'friends' do for one another.
} 
It should also be acknowledged that these dilemmas were kept from supervision meetings during the course of the research. This probably added to the pressures on myself. However, if these had been communicated to my supervisor, then he too would have been faced with ethical dilemmas of whether or not to pull me out of the field - this was a situation I chose to avoid. Full revelation and discussion of the dilemmas occurred after exiting the field.

As it was, the officer group accepted the research project, and me, and provided greater access than I could have anticipated. At the same time, the core principles of academic ethics were met, albeit at times through situational ethical practice rather than the traditional rule-utilitarian manner. Again, this experience was not unique to this project and therefore does not jeopardise its academic integrity (see for example Lee, 1993; Lee-Treweek \& Linkogle, 2000; Marquart, 1986; Whyte, 1984).

\section{Presentation Of Self}

Marquart (1986) aptly asserted that in observational research it was often the researcher who was more the researched than the participants. As the researcher, it was you, rather than the researched, who was centre stage. Every behaviour, utterance and gesture was intensely scrutinized by multiple participants in an attempt to reveal one's supposed true characteristics and intentions. As I was an overt observer, what was required was a presentation of self that facilitated group access while reinforcing the validity of my role as researcher and hence proving invalid any assumptions of alternative roles such as 'official spy'. Douglas (1972) proposed this was the problem of 'face management' that ethnographic researchers must address both in terms of eliminating the front of the participant while projecting an appropriate front as a researcher. As a young white middle class academic female within a disproportionately 'brown' ${ }^{, 44}$ male environment, effective dual face management might seem problematic. However, Easterday, as cited in Morrison (1989, p. 50), suggested being a female observer could have its advantages in that “... not being taken seriously can work to one's benefit. If a researcher is not taken seriously

\footnotetext{
${ }^{44}$ Within the New Zealand context, Maori and Pacific Island peoples are referred to as brown rather than 'black' or 'coloured'.
} 
because she is a young female, this can facilitate entrée into an otherwise difficult or inaccessible setting”.

Similarly, Gilbert (1994) suggests that a useful observational technique for front management was to cultivate an impression of naïveté and humility or the role of 'acceptable incompetent'. In this way, participants were sympathetic and compelled to explain things to the researcher. Therefore, in the first instance, my gender was beneficial in supporting the presentation of self as the 'sociable dumb blonde' enacting both Douglas' (1972) proposed dual fronts while encouraging active participation by participants in the manner of Gilbert's sympathy for the incompetent. This is not an uncommon technique. For example, Glebbeek (cited in Huggins \& Glebbeek, 2003) wrote that her gender and age were advantageous as a mask of naïveté to access a difficult group’s secrets.

This presentation of self did raise issues of deception though, as participants were offering information under the aegis of a masked front. Using the risk-benefit analysis of ethical issues in prison research proposed by Overholser $(1987)^{45}$, a subjective calculation would suggest an acceptable level of deception and hence permissible ethical violation. Further, this presentation of self was an imposed interpretation of me and my ability, proffered by officers. Therefore I was not actively asserting the stance or mask, merely passively accepting it due to its utility and efficacy for attaining access. Regardless, it is arguably the most common technique of observers to assume some form of masked face management (see for example Dalton, 1959) and is certainly less detrimental than other deceptive presentations (see for example Humphreys, 1970).

The 'sociable dumb blonde' role was also effective at reducing other issues that could arise within such groups as prison officers. For example, given the hierarchical nature of the officer culture, it would be difficult for a researcher to be accepted by one group of officers without alienating themselves from legitimate and full acceptance into other groups. The wider societal perception of the 'sociable dumb blonde' as indiscriminately friendly and extroverted provided an acceptable rationale to officers

\footnotetext{
45 This risk-benefit analysis compared the likely risk of harm to the participants against the potential benefits of the mask for research purposes.
} 
for my movement between groups. Arguably, such perceived neutrality relative to internal factions would have been difficult for someone who more readily matched a particular officer group physically or socially, such as a middle-aged male or Maori researcher.

Therefore there were benefits being a young female in the context of the male prison as a research site. However, gender also had associated problems. For example, as a female one was perceived as having a capacity for unlimited and unconditional emotional support. As I became accepted within the group, particularly behind the mask I had chosen to wear, I inadvertently created the need to continue this presentation. Countless hours were spent with male and female officers discussing such non-research topics as relationship problems, child rearing issues and so on (see also Piacentini, 2004). Within the rule-utilitarian approach this might be considered problematic, although as Oakley $(1981,2000)$ argued, it can also be a necessary part of the research enterprise. Within the officer culture, it was certainly an expectation that I displayed an apparent extended interest in all facets of officers' lives. Indeed, it could be viewed positively as a reflection of the level of my inclusion within the group. Regardless, the pressure on my time and energy was at times difficult to manage.

Another negative issue of being a female researcher within a predominantly male environment was the need to contend with unwanted male attention of varying intensities ${ }^{46}$ (see also Huggins \& Glebbeek, 2003; Piacentini, 2004). Discussion with and observation of other women within the prison, including female officers, suggested my treatment was not out of the ordinary. Aligned to the character tests, women were required to manage themselves within this frequently gender-hostile environment. While to non-participants it might have appeared the men were 'flirting' with me, it was more congruous with the gendered control techniques employed by some groups of male officers. This point will be extended further in the thesis, suffice

\footnotetext{
${ }^{46}$ While generalised sexual comments and invitations were expected, forceful sexual advances and stalking type behaviour initially surprised me. For example, one male officer sent numerous bunches of flowers to my home and university office with 'love' messages attached. Another would arrive uninvited at my home or university office — often between $10 \mathrm{pm}$ and $5 \mathrm{am}$ — and would refuse to leave. On one occasion he even forced entry into my home. Mild advances were easily dealt with in the manner that one would 'turn down a date'. More aggressive advances required a confrontational approach with the individual concerned.
} 
to note here that being female enabled me to be more aware and cognisant of female prison officers' experience. In sum, while there were benefits to being a female within a male facility, it could be quite dispiriting at times.

\section{Loci Of Participants}

Dane (1990) suggested observers relied on informants, not merely for data but also for generalised advice on such issues as participant selection (see also Whyte, 1984, 1993). Use of informants, or informally incorporating 'gatekeepers', has similarly been common practice in prison research. For example, Marquart (1986) explicitly detailed the need to use key personnel to facilitate acceptance (for further examples see also Carroll, 1974; Giallombardo, 1966; Jacobs, 1974; O’Connor, 1976). Prior to site entry I had no known associations with officers and hence was not afforded such advice or social access. Therefore, I needed to rely on the face management techniques outlined above while I sought to identify potential gatekeepers.

Several key members of the officer group were identifiable as influential for social access. They were not actively incorporated as informants, but rather as passive collaborators or informal gatekeepers. That is, efforts to provide information about the research were directed at these actors who in turn disseminated this information through the officer group as they deemed appropriate. Once these officers were satisfied with my presentation of self and therefore my apparent motivations and role, the officer group as a whole responded more favourably to the project, participation rates increased and access to the various facets of the prison officer world was granted.

Use of such techniques raised two important issues concerning the nature of the voluntary consent obtained. It was difficult to respond to either, however, without engaging the tautological argument that such an approach was required by the local prison officer culture. In the first instance, it could be argued that individual participants might have been exposed to a degree of external influence from those officers acting as my informal gatekeepers as these members possessed more systemic power. This would mean that they did not have selective control of my access to the 
group and by proxy to themselves (see Altman, 1975). I was aware of this potential abuse of influence and was mindful of the need for the occasional discreet discussion with individual officers to ensure that they felt empowered in the process and sufficiently able to decline participation, although there was little elective nonparticipation.

The second issue concerning the nature of the voluntary consent was that I had not openly negotiated relations with these informal gatekeepers. To assert this as a serious issue, however, would be to assume my primacy within the environment - a position I clearly did not hold. The success or otherwise of the project lay with their perception and acceptance of $\mathrm{me}^{47}$, the process was not as reciprocal as a researcher may intend.

Taking the approach of having informal gatekeepers rather than active informants required a higher degree of involvement in the group than otherwise would have been necessary. Therefore, countless additional hours were spent participating in officer group activities beyond the research proper. Such activities included: formal and informal social functions both on and off site; cultural events such as tangihanga (funerals) and kapa haka (cultural dance festivities); sports events; generally 'hanging around' and chatting after shifts and so on. While data was not collected during these times, data collected during the research proper must have been affected by the generalised relationship between the participants and myself. While this raised the issue of participants' motivations to participate, it is difficult to identify prison field research that does not incorporate and acknowledge the value and validity of such practices (see Wolcott, 1995). Further, it would be problematic to deny some level of relationship development when one engages with a group over an extended period of time. What was important was that the researcher did not misuse or abuse such

\footnotetext{
${ }^{47}$ The following is an example of an incident where a particular group not only 'tested' me, but explicitly assigned approval for acceptance. I was invited to the 'prison village' (an area of Department of Corrections subsidised housing situated near the prison inhabited exclusively by prison officers and their families; for further details of the prison village see chapter three) for a social gathering. During the course of the evening, when the officers assumed me to be suitably intoxicated, they fired a barrage of questions at me concerning the research. I answered qualitatively the same as if within the prison. The following day one of the officers involved took me aside and informed me that I had only been invited so that they could ascertain the genuine motivations behind my project and the veracity to my claims of only being an academic (as opposed to a Department of Corrections employee and hence 'spy'). Given my responses when I was supposedly intoxicated, I had officially 'passed' and was 'alright'. This event marked a significant junction in my research process, after which there was a noticeable improvement in willingness to generally associate with me and participate in my project.
} 
relationships and that roles remained clear. Such issues were repeatedly discussed between my supervisor and myself. Therefore, I would argue that these relationships did not jeopardise the integrity of the research but aided access. Such relationships did raise issues, however, in relation to 'going native'.

\section{Going Native}

This is a concept that engenders varying responses from academics and has attained numerous definitions and associations. An unembellished definition, and one which I contend holds the common basic conceptual elements, was proposed by Martin (2000). He defined going native as the extreme version of the researcher losing objectivity and neutral perspective by identifying with some or all of the research participants. This arises in participant observation when the researcher enmeshed themselves deep within the participant group. It is assumed that in such situations the researcher becomes inextricably involved, thereby obscuring all possible objectivity. The researcher then has a partisan position in relation to the experience of the participants, indeed even of the participants. Whether this was a positive or negative position, it is thought that it deleteriously affects the validity of subsequent theory development and presentation of data.

It has been argued that the risk of going native is extremely high within the prison as a research site due to the insular nature of the environment. My view is that both the environment and subsequent data required in-depth association, and that such requirements do not jeopardise the integrity of the project. This position is not uncommon among prison ethnographers. For example, Liebling (1999) lends support. She describes the nature of the prison as a research site as:

... an intense, risk-laden, emotionally fraught environment. It makes demands on fieldworkers which are at times barely tolerable. The risks of 'going native' are high - particularly when long periods of time are spent with staff and prisoners in 'the deep end' of prison life. Without this exposure, in the intimate 'places' and 'times' of the prison world, the research is superficial. (p. 163) 
I would argue that any qualitative research in social science will, by the nature of the topic, incorporate some form of bias and group attachment. As Becker (1967) noted, to study a group intensely necessarily creates ‘unavoidable sympathies'. For example, on several occasions during the research period I found myself defending the officer group to external agents, even when an officer audience was not present. Similarly, in some circumstances our humanity must necessarily over-ride our role as researcher. For example, as a female I was exposed to particular gender control techniques, directed not only at my sensibilities, but also on occasions threatening my physical and sexual safety. Thereafter, it would be difficult not to identify with or possess unavoidable sympathies for the gendered pains of occupation felt by female officers. Indeed, Oakley (1981) argued the pretence of neutrality was actually counterproductive to the aims of qualitative research and that participation demanded alignment with participants. It was part of the academic enterprise, however, to ensure that such sympathies did not render the results invalid.

For the purposes of this research and the closeness that I achieved with the officer group, I would accept the assertion that I had indeed gone native. I did possess some unavoidable sympathies and could identify with facets of the group. However, I would argue that this did not necessitate a weakening of perspective or bias within the research proper. Similar to Liebling (1999), Gilbert (1994) contended that a greater risk to research than going native was 'not getting close enough'. Ultimately, the data I was seeking would not have been possible without the approach of entering the group.

Further, I would argue there are degrees of going native and that my approach was not extreme. After all, I was never considered a true member of the prison officer group. Instead, with the selected presentation of self, I attained the academically desirable status of 'fictitious membership' as proposed by Douglas (1970). That is, it was known that I was not a member of the group, but for most purposes I was treated as a member. In this way, I was exposed to a level of data reserved for such membership but was, however, able to subsequently withdraw from the scene as I was at all times partially detached due to the fictitiousness of the circumstances. As Gilbert (1994) argued, adopting the perspective of members is a valid methodological tactic and frequently employed, even if not always acknowledged formally. 
Morrison (1989) proposed that participant observation required the suppression or postponement of satisfying personal needs in order to fulfil the field role. I would argue that when one is not truly a member, the energy required for suppressing personal needs and front management could only be managed for a discreet period of time. For me, evidence that I was not wholly subsumed within the culture was that towards the end of the research period the desire to withdraw became overwhelming and it was with relief, rather than regret, that I left the field. Liebling (1999) described the experience of exiting the prison world and re-entering her own world as encompassing “a sense of detachment and disorientation” (p. 161). I similarly felt relieved to be free from the mask and rigours of field research. 


\section{CHAPTER THREE: FROM DU CANE TO ECONOMIC RESTRUCTURING: THE HISTORICAL DEVELOPMENT OF THE NEW ZEALAND PRISON SERVICE}

In order to gain an understanding of the day-to-day experiences of prison officers as they made sense of their work in the aftermath of dramatic organisational change, their current position needs to be located within the historical development of the New Zealand prison service. The most detailed and informative account of the historical development of the prison officer ${ }^{48}$ group is still that provided by Thomas (1972) in his work on the evolution of the English prison officer. By locating the existence of the prison officer group within the historical evolution of the prison system, he demonstrated that regardless of subsequent changes within the prison service or to the supposed role of the prison officer, the presentation of the group and their occupational culture remained the same.

While seemingly pessimistic, the central tenet of his thesis was that despite the prison system's increasing declaration of and support for reformative goals that were then in vogue, the role of prison officers had, and would remain, purely custodial. Indeed, he went on to argue that there had actually been a narrowing of the officer role over time,

\footnotetext{
${ }^{48}$ Throughout this chapter the terms warder and officer were largely interchangeable unless otherwise noted (that is, until 1951). In England the change of terminology demarked specific periods in the history of the English prison officer with the change from warder to officer occurring in 1921 (Thomas, 1972, p. 146). Such demarcation through language usage was not apparent in New Zealand official documentation and demonstrated a non-specific application of the terminology with multiple terms appearing from the outset. For example, as early as The Reports and Memoranda of the Judges of the Supreme Court (Arney, Johnston, \& Gresson, 1861) the terms warder, officer and guard were used interchangeably. Indeed, the term turnkey was similarly used within this document in reference to warders and was not applied in a derogatory sense or to denote specific tasks (as in England). It should also be noted here that in the New Zealand context, due to the low numbers of inmates and diminutive size of facilities, inmates were not co-opted in roles of 'turnkey' as they were in the English system. Therefore all such references were to officially employed prison staff.
} 
even though most other literature ${ }^{49}$ at the time argued to the contrary. Indeed, for Thomas (idem), this challenge to the officers' role that the then emphasis on reform and rehabilitation represented had led to a strengthening of their dependence on and overt presentation of prison officer culture. This happened as officers attempted to assert their utility and importance within the prison system. He argued that his hypotheses, while controversial, were sustainable when one examined the officers' role as relative to and derivative of the social structure and environment within which it resided. The historical development of the New Zealand prison service from the late nineteenth century up to the early 1970s followed much the same course as that set out by Thomas. Thereafter, however, it faced dramatic restructuring.

\section{Bringing Du Cane To New Zealand}

Prior to 1880, the New Zealand prison service had been organised on a very ad hoc, often chaotic basis (see Harris, 1977; Locke, 1978), as might be expected in a frontier society (New Zealand had been colonised by Great Britain in 1840). This had been recognised in the Report of the Gaols Committee (1878) which recommended the appointment of an Inspector General of Prisons to bring consistency and order, along the lines of the role played by Sir Edmund Du Cane in Britain. Captain Arthur Hume was the first such appointment in 1880. Hume came from England where he had trained under Du Cane and followed his mentor's rigidity and severity by introducing and enforcing a strict, militarised regime. This included the progressive stage system of classification, building projects and a marks system for inmate labour (Pratt, 1992). The marks system was significant as it was a graduated pay system for those inmates without employment through to the differing levels of skill and hours worked. The inmate group having a source of income, and requiring staff support to alter their employment status and hence financial return, provided a means by which staff could informally negotiate access to goods and services and thereby assert additional control over inmates.

\footnotetext{
${ }^{49}$ It should be noted that the majority of other 'literature' was typically official documentations rather than academic critiques such as Thomas.
} 
Furthermore, his classification and marks system now increased the authority and power of prison staff by establishing a clear means of asserting incentives and punishment for inmates' behaviour. At the same time, in relation to the conduct of his prison officers, he introduced a fine system to increase the professionalism of the officers' behaviour in a bid to remove their own abuses. This was achieved through a negative reinforcement system for forty nine separate offences which prison officers might commit in the course of their daily work. These ranged from leaving a cell door unlocked to allowing prisoners to pass beyond the prison walls unattended (idem). Penalties ranged from fines, with a maximum of $£ 20$, through to suspension, depending on the severity of the misconduct (Ministerial Committee, 1989).

Although the fines were rarely invoked, the system offered gaolers ${ }^{50}$ a formalised means to assert dominance over officers with a similar style of scrutiny and punishment regime to that which they placed on inmates. Therefore, not only were officers meant to be skilled and proficient in surveillance but, also, they themselves were under a similar form of surveillance from above. What it is likely to have led to is that officers' behaviour became more insular as they became suspicious and mistrustful of senior officers and the prison authorities. Information relating to the conduct of others would become equated with power as officers began to store mental notes about their inmates and colleagues. In these respects, the stage was being built for division, suspicion and resentment between the 'shopfloor' and 'management' staff ranks, while surveillance of everyone in the prison became part of the operational logic.

In addition, Hume preferred to recruit new applicants with armed forces backgrounds. In the manner of Du Cane ${ }^{51}$, he claimed they would be more successful at instilling military style discipline. As such, by the time of his 1889 annual report, Hume (1889, p. 3-H.-7) was able to comment that "[a]ll fresh appointments to the prison service are now made from men serving in the Permanent Militia”. The result was a work force that was more highly disciplined, more regimented and trained across the whole

\footnotetext{
${ }^{50}$ The term gaoler can be equally substituted in this context with 'governors', then 'superintendents', then 'manager custody', depending on the time in history as the position was the same.

${ }^{51}$ Du Cane, cited in Thomas (1972, p. 47), said of military recruits: "Their [ex-soldiers] (sic) habits of order and discipline, of rendering and enforcing strict obedience and their aptitude in dealing with large bodies of men, are unquestionably very valuable qualities for the office, and if not possessed by an officer on joining, would have to be acquired more or less perfectly afterwards".
} 
prison system, while also being typically white, urban and respectable working class men. The uniformity amongst these officers was then conducive to greater collegiality and the subsequent development of a group culture, as Hume (1884, p. 3-H.-5) noted:

\begin{abstract}
It was stated in my last report that numbers of the officers did not sufficiently study the characters of the prisoners under their charge. A melancholy example of this occurred in the Nelson Prison in the murder of a warder by a prisoner, and the suicide of the murderer.... A pleasing circumstance connected with this fatality, and one which affords me gratification to record, is that the officers of the department all over the colony raised by subscription among themselves a sum of upward of $£ 290$ for the benefit of the widow and family of the murdered man. \{my emphasis\}
\end{abstract}

\title{
A Shift From City To Country
}

On Hume’s forced retirement in 1909 (like Du Cane before him, his militaristic approach to prison discipline was now thought to have run its course), Sir John Findlay became Inspector General of Prisons. His vision included reformation through productive labour and a system centred on correctional care (Ministerial Report, 1910). To affect such ends, lands were purchased around New Zealand for the development of prison farms ${ }^{52}$ (Department of Justice, 1914). The move to prison farms not only shaped the nature of New Zealand prison industries ${ }^{53}$ but also the shape of the officer group as prisons came to be increasingly situated in rural areas or the outskirts of towns to accommodate the agricultural focus. The physical movement from towns and cities further contributed to the insularity of the officer group as they only had their peers to socialise with. The closure of urban facilities and establishment of rural prisons continued to be central to prison policy throughout the next century and few institutions are now located within city limits. As this happened, some of the older, 'makeshift' gaols were closed.

The higher concentrations of staff within a smaller number of institutions then strengthened the potential for the development of a collective culture. Not only this, but with the increased isolation, the officer group became increasingly homogenous.

\footnotetext{
52 The target prison site was one of these early prison farms.

${ }^{53}$ For example, Corrland Inmate Employment's forests and farms contribute towards most of the \$20 million earnings from inmate industries, which is set against the Departments budget of \$440 million per annum (Department of Corrections, 2003).
} 
While they remained predominantly white, male, ex-military and respectable working class, a significant change was brought about by the shift from city to country prisons. New recruits were increasingly rural people rather than townsfolk. This provided two subtle but important shifts within the officer group. First, local rural recruits tended to be more physically commanding, due to their previous employment on farms, than their urban office-based counterparts had been. Secondly, rural-based males placed emphasis and reverence on the New Zealand ideology of the Number 8-Wire. The 'number 8-wire' philosophy within New Zealand culture derived from the notion that anything could be fixed using 'number 8-wire' and a little pragmatic imagination ${ }^{54}$. Number 8-wire was a general wire that was used predominantly in farming, but a similar philosophy began to make its mark in prison administration. In this way, preference for practical skills rather than educational knowledge became another feature of the officer group. It was thought the example they set by their demeanour and bearing would itself have a positive influence on the prisoners. For example, Ironside (1910, p. 9), the Gaoler of Lyttelton Prison, noted the need to recruit officers on the basis of them being "men of high moral character, good physique, competent to obtain discipline, and at the same time contribute to the reformation of the prisoners by their good sense, tact, and example”.

Another significant development for officers at this time was the acceptance and implementation of Gaoler Ironside's (1912) recommendation that, to retain staff of suitable quality, remuneration should be higher than that which they could attain in ordinary employment. While specifics were not documented, it was clear that this recommendation was taken note of, as the Hay and Matthews (1913) report to the House of Representatives referred to a noticeable improvement in the supply and quality of recruits due to the amended salary scale and reduced impediments to salary increases.

The growth of prison 'villages' in the aftermath of the 1910 reforms was very significant in the light of prevailing social conditions and indeed produced recurring job benefits. The new positioning of prisons led to the clustering of state owned houses in their vicinity. These charged considerably less than market rentals and were

\footnotetext{
${ }^{54}$ For more on the influence of the 'number 8-wire' philosophy on New Zealand culture see James and Saville-Smith (1990).
} 
exclusively tenanted by prison staff. Indeed, at their de-commission in the 1990s, rentals were around $10 \%$ of local market value. The prison villages did not just provide low cost accommodation. In addition they increased the attraction of the job; removed the chance of staff lodging within areas inhabited by 'undesirable' populations; created a community of supportive, similarly situated families ${ }^{55}$; and fostered a sense of security among the staff while at home and at work. In regard to the last, there was now a ready supply of officers who were always nearby in case of disorder in their institution. For the officers within the villages, the prison came to dominate both their professional and personal lives. They were invariably detached from many external influences and this allowed growing commitment to the prison while increasing suspicion of those from outside their homogenous communities. Indeed, this point was eventually noted by the Ministerial Committee (1989, p. 84), which recommended the closure of the prison villages: "[these] lead to the undesirable separation and isolation of prison officers from the rest of the community. This works to the disadvantage of the officers and certainly their families". Meanwhile, the expansion of the agricultural programme had increased self-sufficiency of prisons to $40 \%$ of the cost of rations by 1933 (Newbold, 1989). This meant that the conditions of the prison system, including those of the staff, suffered less than the rest of New Zealand society during the economic hardship of the depression and war years.

It can be seen then, that after the centralisation of control and generalised uniformity that followed Hume's appointment, an arrangement of mutual benefit had arisen within the service and the foundations for a prison officer culture, similar to that in England, were laid. In exchange for support and loyalty to the system, as demonstrated through such characteristics as longevity of employment (Pratt, 1992), reciprocal obligations to fellow officers without expectation of payment (Newbold, 1989) and general good-will and stability, working as a prison officer could have significant attraction, especially for low-skilled workers in the depression years. At the same time, it was a straightforward and uncomplicated occupation. An officer was responsible for locking and unlocking, supervision of routine activities and preventing disturbances and escapes (idem). Communications between inmates and staff were officially restricted and formalised. Measuring achievement of these goals was

\footnotetext{
${ }^{55}$ Non-married employees were housed in a designated 'singles quarters' building thereby creating a pseudo 'family' unit.
} 
simple. As there were few reported escapes during this pre-1950 period, officers were deemed to be 'doing their job'.

However, in the immediate post-war period, there were a number of well-publicised complaints about prisoner mistreatment (see for example Burton, 1945; New Zealand Parliamentary Debates, 1945). Officer morale seems to have dropped at this time as inmate recalcitrance ${ }^{56}$ increased and the general public became critical of the system, as evidenced by a barrage of 'letters to the editor' in the New Zealand press. Newbold (1989) argued that as the Department of Prisons did not respond to this fervour, officers interpreted the inaction as central office being disinterested in the effect on staff and a significant number of resignations ensued. Thereafter, New Zealand society as a whole enjoyed considerable prosperity in the post-war period and with many other employment possibilities now, working as a prison officer began to lose its attractions. The Public Service Association ${ }^{57}$ (PSA) lobbied for change to increase staff retention and make the job more attractive to new recruits. Although an improved salary scale had been introduced in 1946 with a designated forty hour week (previously shifts had lasted 12-13 hours) between 1945 and 1951 there was a further $22 \%$ decrease in officer numbers raising the ratio of officers to inmates from 1:4.5 in 1945 to 1:6.0 by 1951 (see Appendix H). At the same time, the officer group which remained was coming to depend on the simplicity of their existence as the main attraction of the job: higher wages in an era of minimal unemployment could easily be found elsewhere. In contrast, the prison officers were a homogenous and insular group, poorly paid but unified through longevity of service and communal life in the villages.

\footnotetext{
${ }^{56}$ These were predominantly conscientious objectors' protests as these were the first inmates in New Zealand politically motivated to act. They created disturbances in the jails, generally by passive protests such as hunger strikes and sit-ins, over perceived ill treatment of inmates by staff.

${ }^{57}$ At this time this was the only union addressing the needs of prison officers.
} 


\section{Back To Security And Surveillance After Rehabilitation}

The period from 1925 to 1949 had produced little change in New Zealand prisons. In the 1950s, however, the new Head of Prisons, Samuel Barnett, took the view that prison work still bore the imprint of the Hume era. As such, it was highly restrictive with not enough emphasis on reform for inmates or professional development of staff. He set in motion a revision of the Prisons Act 1908 which produced the Criminal Justice Act 1954 and Penal Institutions Act 1954. These led to significant revision and refocus in the area of inmate reform and training. To affect these changes, full-time chaplains, trade teachers and schoolteachers were introduced to each prison (Ministerial Committee, 1989). Full on-site medical services were established including nurses, doctors and basic dental services.

Furthermore, in A Penal Policy for New Zealand, the Department of Justice (1954) revised the classification of inmates. Local sub-committees were established with the responsibility to prepare reports on each inmate which were then submitted to the Classification Board. This board was established to review each inmate's case and make submissions to the appropriate prison administrations with suggested plans for use of the inmate's time to aid in their reformation. In the process of preparing this report, local sub-committees were able to call upon an array of professional support personnel such as psychologists, psychiatrists, vocational guidance officers, probation officers or any other specialist service of assistance in defining the offender's character and potential. Prison officers were not, unless specifically called upon, included in this consultation process. Such developments clearly point to a very significant realignment of power relations within the prison. The primacy of noncustodial specialists was firmly asserted over and above that of prison officers. Almost as an afterthought as it was on the last page of the report, prison officers were co-opted into the new framework of reform:

He is expected to study the man, to read his case records, and to appreciate in what ways he differs from another; he will be called upon to furnish to the Classification Board, and to the after-care officer prior to the prisoner's release $a$ report not only upon the prisoner's conduct, but also upon such matters as his reactions to imprisonment, his relations with other prisoners and the staff, his attitude towards crime, his character, potential, strengths, and weaknesses. 
These reports are becoming progressively more important and they have a direct bearing upon the decision as to the prisoner's suitability for release. \{my emphasis\} (ibid, p. 31)

In this way, the existing ability of the prison officers to collect information on inmates was actually reinforced even if, formally at least, this was for the purposes of rehabilitation rather than surveillance, as it had been before. Overall, though, the 1954 Report downgraded the importance of prison officers' work in the intended reformative process. From playing a central role on the prison stage, it seemed that they had suddenly been relegated to relatively inconsequential stagehands, as all the new treatment staff arrived.

Coinciding with the introduction of the 'professional' personnel to the prison, inmates became increasingly involved with community groups such as the Salvation Army, and also began to have more contact with the world beyond the prison through sporting/ cultural events and home leave opportunities (see for example Newbold, 1989; Ministerial Committee, 1989). The increased movement of inmates between the prison and outside society thus challenged the importance of security and surveillance and hence the legitimacy and value of what officers still perceived to be their primary role. More importantly, introduction of the new professional personnel challenged the idea that prison officers 'knew' the prisoners and understood them better than any other professional group. Inevitably, such developments enhanced not only the officers' sense of isolation, but also their solidarity as a threatened group. As such, the only means by which prison officers could assert power over the new professional staff was to limit the information they provided to them. The acquisition and transference of knowledge became a commodity within the prison system that could give officers power if they mastered and manipulated it. In an attempt to enhance staff morale, professionalism and overall attractiveness of the service as a career option, Justice Minister Barnett introduced a training school in 1951 to be attended by all new recruits for four weeks in Wellington, and officially changed the term 'warder' to 'officer' for custodial personnel ${ }^{58}$ (idem). Attempts at professionalisation and concern

\footnotetext{
${ }^{58}$ As outlined previously, within official documentation these terms had been used interchangeably and with non-specific inference. Regardless, the official demarcation of the term 'officer' in 1951 did denote an attempt at professionalising the staff.
} 
about the public image of the prison service continued, as demonstrated in the 1954 annual prisons report:

\begin{abstract}
If we are to attract men to the service we must increase the regard in which prison officers are held by the community. At present the prison service is not held in a high enough esteem; the past still casts its shadows, and to too many people the prison officer remains the turnkey. There is no place in a modern prisons administration for that caricature of the unintelligent, possibly sadistic, man who was a turnkey only because he had not the wit or the ability to do any better for himself. (Department of Justice, 1955, p. 13 - H-20)
\end{abstract}

Whereas 'warder' had connotations with nineteenth century 'turnkeys', 'officer' now suggested someone in the position of 'leader' (see Fox, 1952). Dissatisfaction and low morale was also partially addressed with a wage re-adjustment. During the latter part of the 1950s payment for non-routine hours (overtime) was also introduced (Sarr, 1995).

A final important development arising from the Department of Justice (1955) annual report was an appreciation of the potential influence of prison officers' wives and families to the morale of staff and as a means of encouraging longevity of service. Funds were allocated for building additional married accommodation; transporting wives to nearby shopping centres; building social halls and children's playgrounds; and generally providing amenities for the contentment of the officers' families. These initiatives had a substantial influence on the developing officer culture. The officers' wives, colloquially referred to as the 'village wives', were effectively accorded a role within the officer group. As information gathering increased in importance amongst the officers, wives similarly became involved in such tasks. However, the wives' surveillance could only be on other officers and their families, given how much of their time was spent in the prison village. The subsequent transmission of this information to their husbands led to the development of the importance of information gathering on fellow officers as well as inmates. Over time, the village wives increased their influence and power within the officer group and could be fundamental in the acceptance or otherwise of outsiders or newcomers.

The direction of prison policy then changed after a number of inmate insurgences, escapes and hostage takings between 1958 and 1965 (Commission of Inquiry, 1965a, 
1965b). The exceedingly high staff to inmate ratio of 1:9.0 (see Appendix H) eventually led to recognition that there was significant pressure on the officer group. In response, the prison authorities imposed a tightened disciplinary regime, including the building of New Zealand's first maximum security prison.

At the same time, as a further attempt to change the public image of the service, the government introduced a cadet-training scheme in 1967 (Cameron, 1973). It was thought that a residential training centre for school leavers with aspirations of a career in prisons would remove the impression that entrance was relatively unrestricted, and lead to the prison service being perceived as a viable career option. Equally, it was thought that it would support a culture of training and professionalism amongst the existing staff group, as it would be shown that it was not the case that 'anyone' could be a prison officer. However, these attempts were without effect and the cadet school was soon disestablished ${ }^{59}$. Similarly, it would appear there was little increase in enthusiasm for additional training within the existing staff ${ }^{60}$. It would have been difficult to achieve this end, given that unemployment was low and, as public sector employees, prison officers had secured numerous benefits, including unconditional tenure and substantial financial benefits regardless of rank. Therefore, training was limited to the minimum required to gain promotion, and status within the service would be achieved as a matter of course by upward mobility through the ranks. There was no real incentive for officers to train for the sake of it. Mere length of service would ensure promotion to at least second officer status (see Figure 1.1 and Appendix $\mathrm{K})$.

At the same time, the service was very hierarchical, providing much scope for promotional opportunities, largely based on length of service. The low level of qualifications or additional training required of officers, transparent promotion potential, multitude of state sector perks and privileges and job security combined to

\footnotetext{
${ }^{59}$ The cadet school was disestablished in the early 1970s period. The reason for the cessation of the scheme was not documented, however, given most literature directs to the prison service being a secondary career option it was unlikely that many 17 year olds would have been interested in pursuing a career as a prison officer (see for example Crawley, 2004; Jacobs, 1978; Jacobs \& Retsky, 1975; Jurik \& Halemba, 1984; Kauffman, 1985; Lombardo, 1981; Morris \& Morris, 1963; Myers, 1995).

${ }^{60}$ There were no official records of internal training provision to measure this on. However, prison budgets did not change to indicate additional training during this period and anecdotally there was no change throughout the twentieth century.
} 
make prison work an attractive option at that time for the white, working class males whom it largely employed. Indeed, between 1966 and 1971 there were staff increases of $225 \%$, bringing the prison officer to inmate ratio down to 1:3.7 (see Appendix $\mathrm{H}$ ). This noticeably enhanced the conditions of their employment even further. Surveillance and security had returned as the most crucial aspect of their job, permitting simplicity of task and expectations. The rank structure provided clarity of communication and responsibility.

As a consequence, by the 1980s prison officers enjoyed high levels of solidarity and remained largely in control of the prison system. As a public sector body of workers they had also been able to accrue significant perks that supplemented their basic wage while enjoying an almost guaranteed job security and tenure. However, this taken for granted permanence was to be severely disturbed, not just in the prison service but also across the whole of New Zealand society. The 1980s saw a deliberate and systematic deconstruction and rebuilding of the New Zealand state (Walsh, 1991).

\section{A New Way For The Prison Service: 1980s Restructuring}

Economically, post-war New Zealand had enjoyed one of the highest standards of living in the world and seemed the embodiment of security and stability. Furthermore, successive governments had been prepared to intervene to offset any disturbance to this tranquillity: unemployment, for example, was kept to a minimum by strict policies of demand-management and protectionism (Massey, 1995). Nonetheless, the rigorous state regulations necessary to bring this about had the effect of suppressing market mechanisms and required high levels of government expenditure and taxation to sustain them. This led to a gradual decline in real living standards relative to other developed countries and dissatisfaction amongst the general public. In 1984, the Labour government began a process of economic restructuring with generalised goals of deregulation, commercialisation, corporatisation and privatisation, leading to a more internationally competitive New Zealand (Kelsey, 1993). There was, it was 
claimed, no alternative, and for better or worse, the lives of all New Zealanders were dramatically affected.

This country was not unique in experiencing considerable economic restructuring and development during the 1980s. What was significant in New Zealand, however, was the degree and rapidity of change. Within a few years, New Zealand moved from being the most insulated and government protected economy of the OECD to a country that experienced the most radical economic reform programme in the developed world (Shaw, 1999). The effects of this were heightened as, prior to this period, the combination of the high quality of life, lack of obvious social turmoil and physical isolation from external influence had allowed New Zealanders to develop a complacency and belief that 'the world would always stay the same' (Kelsey, 1997). Few were prepared for the level and severity of the changes and challenges ahead.

Although there were many benefits to the new economic climate, such as deregulated import policies enabling unprecedented consumerism, there were also many adverse consequences. State sector employees were amongst the most severely affected because the employment environment that had emerged during the preceding regime had particularly advantaged them. For example, state employees had benefited from complete job security with unconditional tenure and progressive fixed-pay-scales that were generally only dependent on service time (Massey, 1995). Essentially, state employees could not be required to fulfil any particular work objectives, nor could they have their employment terminated, and as long as they continued to report to work they would continue to receive pay increments. The undemanding nature of the working practices of public sector employees was ironicised in the long running situation comedy ‘Gliding On’ (Inside Television New Zealand, 2007).

However, these arrangements were no longer tenable with economic restructuring. The State Sector Act 1988 and the Public Finance Act 1989 dismantled, overhauled and reconstructed the public sector in closer alignment with principles of private enterprise. For employees, this meant private sector working practices would now be applied (Shaw, 1999). All of the previous taken for granted privileges of the public sector effectively disappeared 'overnight'. The neo-liberal form of governance that replaced the previous highly bureaucratised mode of welfare governance meant that 
state employees could now have their employment terminated or be declared redundant, and pay adjustments were to be based on measurable performance objectives and skills value (Kelsey, 1997). The entire nature and character of the public sector was transformed from purely service and personnel concerns to fiscal prudence and profit considerations (Walsh, 1991).

Job security had long been the primary benefit of state employment but was now a thing of the past. Gliding On became no more than an historical anecdote and was soon taken off the air. This, though, was merely the beginning of the paring back of state employee conditions of employment. For the next decade, to meet expenditure targets imposed at the departmental level, public service workers were subjected to a series of negative pay adjustments and reductions in overtime and penalty rate provisions (Kelsey, 1997). Employee manipulation of perks and privileges became impossible, as these simply no longer existed. For many public sector workers this effectively halved their take-home pay.

These imposed changes were only achievable at this time because the previously militant union culture had been systematically weakened to the point of ineffectiveness. Voluntary unionism had been introduced in 1983 following two notable lockouts, which had demonstrated to employees the power shift from unions to organisations that was occurring (Shaw, 1999). Lockouts were not new, however, in the past, the use of such tactics by employers was very rare. It was thus reflective of the change in times that lockouts became the frequent answer to threats of organised industrial action. As union power began to weaken, the Labour Relations Act 1987 required rationalisation of the union structure while practical negotiation obstacles were established in the Employment Contracts Act 1991. Such obstacles included the requirement of every member of a union signing lengthy documentation to authorise their union to negotiate on their behalf. This was required each time the union wished to negotiate. As a final blow to union power, enterprise bargaining saw the creation of new workers' associations, further cannibalising established groups such as the PSA.

Therefore, on an individual level, state employees' perks and benefits had declined, they were now accountable to specified performance objectives, their tenure was no longer secure and their union was essentially ineffective in protecting them. They were 
also expected to embrace the new philosophy of the corporate model at an organisational level which involved new modes of working, managerialist language, equal employment opportunities and potential privatisation of their various facilities and services.

It was little wonder that state employees felt aggrieved at the changes, and no state department was left untouched. Each unified service ministry was dismantled and replaced with a series of independent departments with more defined performance objectives and accountability lines (Shaw, 1999). The Department of Justice was reestablished as the Ministry of Justice, Department of Courts and Department of Corrections (Walsh, 1994). Furthermore, each independent department was divided into subgroups by service. For example, the Department of Corrections, while maintaining this titular label for Head Office, was subdivided into separate accountable units of Psychological Services, Probation Services and Public Prisons Service (with the implication that in the future there could be a 'Private Prisons Service'). Such radical structural change required the new departments and divisions to formulate transparent and measurable strategies for their particular way forward within this new corporate environment. To this end, the Public Prisons Service commissioned the Ministerial Committee (1989) to begin the process of developing 'the new way' for the prison service. As was noted in the introductory chapter, Mr. Kim Workman was brought into the Department of Justice to implement the change process as it related to prison officers. We have already discussed his plans for dramatically restructuring the prison service. Let us now consider in more detail some of their broader dimensions and implications. These included:

\section{(i) Regionalisation And Unit Management}

Following the principles of corporatisation that dominated the state sector during the 1980s, prisons became regionally organised ${ }^{61}$. On one level this was an attempt by the

\footnotetext{
${ }^{61}$ Three prisons, of which the target institution was one, began a pilot scheme to this end in 1984 (Department of Justice, 1988a). The final stage of the regionalisation process was not complete until 1997, at which time the nations prisons had been clustered into eight distinct regions (Department of Corrections, 1997g). The philosophy of regional reception of inmates, however, had been the norm since 1985. The distinction by 1997 for officers was that they were no longer considered a national
} 
government to correct the poor economies of scale that had previously been allowed to develop whereby few penal facilities held more than 150 inmates. Within the new arrangement, services could be directed according to a group of prisons, reducing the overall contribution from Head Office. At the same time, responsibility for budgeting could be devolved from Head Office to the regional level, then further down to the site level. In this way, each state prison could still be considered an individual unit which could be required to compete with private prisons for contracts to take inmates (Unauthored, 1994a). The prison system had been thrust into a supposedly competitive market, contributing to staff feelings of insecurity that were already developing within the changing climate of state sector employment.

The immediately noticeable feature of regionalisation for staff was that they were no longer considered employees of the Department of Justice with assignment to one designated prison. Rather, they became employees of their Regional Prisons Division and an officer could effectively be deployed to any prison within their region at any time. This created a degree of uncertainty and instability for officers, particularly regarding such issues as home ownership and children's schooling, as the other prisons within one region were likely to be geographically distant. The benefits of being a prison employee for family security and stability were rapidly diminishing.

Unit management further decentralised the prison service. The managers were initially appointed in an 'acting' capacity from the ex-divisional or first officer ranks. An attempt was made to co-opt staff participation in the management of change with three 'Unit Management Project Officers' appointed from within the prison officer ranks. These officers were to assist and support designated institutions through the change process. However, the selection process for the project managers failed to take into account the importance of informal status within the officer group. Although full implementation of unit management was achieved by the late 1990s, it had subtly placed the units of an institution into competition with each other for resources. While it was intended that unit management would increase teamwork within units, the effect of institutional competition decreased associative or supportive work arrangements within the generalised staff group. Although an indiscernible shift during group, but were employed and dealt with at a regional level. See Appendix F for the eight regional areas. 
implementation, this was to have significant effects on the social dynamics and power relations within the wider officer group.

\section{(ii) Correctionalism, Case Management And Managerialism}

The Penal Policy Review Committee (1981) had rejected the concept of rehabilitation, arguing that this should be replaced by 'humane containment' and the reduction of the deleterious effects of imprisonment. This minimalist approach to prison purpose was taken further by the Ministerial Committee (1989). It re-evaluated the conditions and circumstances that provided humane custody and 'constructive' sentence use. It was the latter theme which produced the concept of 'habilitation' to replace rehabilitation. This was not merely a semantic play on words. It was contended that rehabilitation required inmates to return to a previous non-offending state, a restoration of former capacities. In contrast, habilitation removed the assumption that any given inmate had been socialised according to societal norms, and sought to equip the inmate and make them fit for life, essentially a transformation to a non-offending disposition rather than a return to a state they likely never possessed (idem). It proposed that this correctional work could be carried out by prison officers in the form of case management, rather than by specialised personnel. Not only was this fiscally responsible, it was also intended to provide officers with more meaningful work than their preoccupation with surveillance and control.

For the prison officers, case management translated to each officer within an accommodation unit being assigned between four and six inmates as their 'case load' for which they were responsible. Case officers were to assess the inmate, propose a constructive sentence plan with programmes and work objectives, initiate access to programmes, advise the inmate on sentence matters, prepare parole reports and so on. To achieve this end meant a significant departure from the traditional relations between officers and inmates, effectively a shift from co-existence to collaboration. Now, officers needed to take an active role in the sentence management of their inmates, a marked change in daily dynamics and tasks. At the same time, officers were not provided any training to achieve these ends, nor monetary incentives, nor promotional opportunities as reward. Their motivation was to be job satisfaction, as if 
at the heart of each officer there was a philanthropic idealism yearning to be unleashed.

The new generation podular prison architecture, while beneficial for unit management, was also helpful in supporting these new relational arrangements. For example, the open environment required a more proactive response from officers in regard to interaction with inmates. Ostensibly, this would help breakdown the existing boundaries and facilitate case management. The greater visibility of the entire confines was intended to generate enhanced feelings of ease amongst the inmates, who should then adopt less defensive strategies in their interactions with both inmates and officers, thereby harmonising relations. Further, spatial distinctiveness of each unit was to engender feelings of ownership and pride of place by both inmates and officers, contributing to the ethos of unit and case management. Such feelings of ownership could certainly be seen at the target facility as inmates and officers were heavily involved in the aesthetic elements of the compound. For example, the inmate welfare committee ${ }^{62}$ in each unit initiated, with officers' support and cooperation, the purchase of additional equipment for their compounds such as basketball hoops, trampolines, flowerpots and picnic table sets.

Notwithstanding these closer arrangements with inmates, managerialism inadvertently distanced prison officers from other groups by rewriting their everyday working language in such a way as to make it virtually impenetrable to outsiders. As the notion of correctionalism became the new focus, the language of the prison began to reflect the new managerialism it represented. For example, prisons were no longer about punishment, but about corrections; similarly inmates were to be referred to as clients, a concept that prison officers, for obvious reasons, found objectionable. Actuarial

\footnotetext{
${ }^{62}$ Each unit had an inmate welfare committee that organised a collection of $\$ 1$ per week per inmate for the duration of their stay within the unit (these monies were non-refundable if the inmate was transferred or released). The money was automatically deducted from their prison account (colloquially referred to as their 'P119 account' due to the association of use when purchasing items through the prison canteen which was done on a form labelled 'P119') and was used to finance group activities such as 'whanau (family) days', the purchase of additional equipment, even the occasional raffle for prized items such as fried chicken and ice cream. This system was similar to inmate councils in England, however, they had no actual influence on policy or procedure within the prison so was not a formal co-option of power. Officers could apply co-option to the situation, however, by permitting or withdrawing their cooperation for ventures. As an example, for whanau days, inmates would negotiate amongst themselves as to what entertainment and food would be provided (such as inflatable castles, clowns, food items beyond the usual daily allowance such as fizzy-soda and so on). Then it was a negotiation with the officers that secured the purchase and delivery of said items.
} 
methodologies were introduced, further reducing the need for specialised personnel within prisons as officers could be reasonably expected to complete 'tick-box' forms. Prison language became littered with acronyms such as $\mathrm{RI}^{63}, \mathrm{RQ}^{64}$, RoC/ RoI, IOM ${ }^{65}$ and $\mathrm{CNI}^{66}$. For outsiders, the constant flow of acronyms, coupled with general prison argot terms such as tealeaf, made prison-speak increasingly indecipherable. If it created new perceptions of expertise and professionalism, it also contributed to the exclusion of non-prison people from the officer’s day-to-day group interactions.

Further, this new mode of working required greater flexibility than was permitted within the Penal Institution General Orders (PIGO) document that had effectively translated regulations into acceptable practice for officers for decades. This document was redeveloped and renamed the Policy and Procedures Manual ${ }^{67}$ (PPM), which was implemented in 1994 (Department of Justice, 1995). It was designed to document all work procedures and specified standards against which the division measured its management performance. For prison officers, however, PIGO had set clear, indisputable guidelines of practice, whereas PPM only provided parameters which could be interpreted widely. Each region, each prison, indeed each unit, implements the interpretations of these parameters differently leading to inconsistency, a feature

\footnotetext{
${ }^{63} R I$ is the acronym for 'Recidivism Index'. RI quantifies the rate of re-offending of a specified group of offenders over a defined period following release using internationally based definitions (Department of Corrections, 2001b).

${ }^{64} R Q$ is the acronym for 'Rehabilitation Quotient'. RQ is a means of measuring the effectiveness of rehabilitative and other interventions in reducing re-offending. The measurement involves a comparison between a group receiving the intervention and a control group that does not. The measurement has two outcomes, RQ reimprisonment or RQ reconviction (Department of Corrections, 2002a). Colloquially these were referred to as the RoC/ RoI (Risk of reconviction/ risk of reimprisonment) indices.

${ }^{65} \mathrm{IOM}$ is the acronym for 'Integrated Offender Management'. IOM is an approach to managing offenders from reception through to release, hence the central activities are induction, assessment of needs and sentence management (Department of Corrections, 1999a). This is achieved through the computer system similarly named IOMS (Integrated Offender Management System; ibid, 1997h). The system links information between the Public Prisons Services, Probation Services and Psychological Services (ibid, 1999b) making it possible for prison officers to track inmates they are interested in throughout the system.

${ }^{66}$ CNI is the acronym for the 'Criminogenic Needs Inventory'. This is an actuarial document that is created by the identified factors that contribute to an offender's offending, derived from the information contained on IOMS (Department of Corrections, 2002b). The inventory is used at the beginning of an inmate's sentence to provide information, within the case management structure, for identification of suitable programmes.

${ }^{67}$ This document guides the day-to-day activities of prison officers and inmates. It is to be read in conjunction with the provisions of the Criminal Justice Act 1985, the Penal Institutions Act 1954 and Penal Institutions Regulations 1961. It is considered a 'live' document and regularly updated (amounting to memos being distributed throughout the prisons and units to be put in the relevant place in the folder). A subsection at the beginning provides avenue for users to recommend changes or updates (Department of Corrections, n.d.).
} 
of prison life which officers abhor. Ironically, the officer group perceived themselves as the experts within the prison and had desired greater flexibility of work practices than allowed under the traditional arrangements. However, the reality of the flexibility that was provided to them under the new arrangements was to become very disturbing as it affected the nature of longstanding social relations within the prison.

\section{(iii) Affirmative Action Recruitment}

The State Services Act 1988 applied the provisions of the Labour Relations Act 1987 to the state sector. This meant that, amongst other things, the Department of Justice was required to implement equal employment opportunities (EEO). The 1988 Department of Justice Statement of Intent and Purpose, as cited in Department of Justice (1988a), asserted a commitment to EEO policies and practices and identified four groups as requiring targeted recruitment, namely: women; Maori; people with disabilities ${ }^{68}$; and other ethnic minorities. Hence the advertisement at Figure 1.5. At the same time, the prison system was then experiencing a period of significant facility expansion. Increases in inmate numbers had reached a crisis point in the late $1980 \mathrm{~s}^{69}$. Initially, transferring inmates throughout the country for accommodation in holding cells and other makeshift arrangements dealt with this. However, a more permanent solution was required - hence rapid facility expansion in the early 1990s. Increased numbers of units required a large influx of staff. What was not considered, however, were the potential effects of sudden demographic change on the already shaken prison officer group. Regardless, affirmative action recruitment ensued.

Women had only begun to be employed as prison officers in male penal institutions in 1985 (Sarr, 1995), with significant resistance from male prison officers. The Ministerial Committee (1989, p. 76) stated that submissions regarding the opposition to women were frequently based on the assertion that:

\footnotetext{
${ }^{68}$ Although there are no formal policies that prevent people with disabilities applying for a position as a prison officer, it is unlikely that such an appointment would be considered appropriate. It is likely that this 'intent' was in relation to non-prison situated personnel.

${ }^{69}$ See figure G.1 in Appendix G for graphical illustration of the increase in inmates per 10,000 mean population and table G.2 in Appendix G for the numerical increase.
} 
... women were not regarded as being capable of undertaking all duties, such as strip searching, and that they were likely to put male officers in danger because they were more vulnerable to being overpowered or taken hostage by inmates $^{70}$.

The Committee, however, was determined to increase the number of women prison officers. However, when this research began in 1997, the complement of women officers at the target institution was still only $17.7 \%$ of the total officer group.

Maori officers were similarly under-represented in general prison officer populations as a result of historical recruitment techniques and the closed homogenous white officer group. While Maori represented around 40\% of prison inmates in the 1980s, only $20 \%$ of prison staff identified as Maori (idem). In an attempt to ascertain Maori prison officer concerns, and elicit factors that might be putting Maori off from joining the prison service, Workman initiated separate $\mathrm{Hui}^{71}$ for Maori and non-Maori staff. This also facilitated the development of Maori cultural awareness initiatives with the intention of reducing ethnic bias within the homogenous 'white' group. For example, in 1994 Te Iho, a Maori based cultural awareness programme, became compulsory for all prison staff. Increasing the Maori staff complement and general cultural awareness programmes for staff was met with hostility from many of the existing staff (idem). By 1997 the target institution had had a significant increase in the proportions of ethnic minority group numbers with $41.8 \%$ of officers identifying as non-Pakeha ${ }^{72}$. However, there was also a clear division between non-Pakeha and Pakeha staff with each perceiving the other group as advantaged. The non-Pakeha category drew a clear distinction between Maori, Pacific Island and Pakeha officers, while the Pakeha staff grouped Maori and Pacific Island officers together as their oppositional other.

\footnotetext{
${ }^{70}$ Interestingly, during the Christchurch Prison (formally known as Paparua Prison) hostage taking in 1997, more male officers than female were taken hostage and it was the female officers that were released during the early stages of the protest (as it was not 'manly' of the inmates to hold women) while the male officers were held for the duration (Bates \& Venter, 1997; Unauthored, 1997a).

${ }^{71} \mathrm{Hui}$ is the Maori term for the gathering together of people for a specific purpose (Tauroa \& Tauroa, 1994). In this case, the gathering was to discuss Maori cultural issues and concerns amongst staff.

${ }^{72}$ This figure represents 32.9\% Maori and 8.9\% Pacific Island peoples from the total officer population. Historical figures of ethnicity were not available, however, it seems reasonable to deduce from the proportions of Maori and Pacific Island staff in the 4-10 year category, coupled with anecdotal accounts from staff, that the figures from this research indicate an increase.
} 
Notwithstanding the opposition to change from the existing prison officer group, the Department of Corrections (1996a, 1997i, 1997j, 1998b) formally adopted EEO and affirmative action recruitment. The introduction of women and non-Pakeha officers was to have a profound effect on the previously homogenous structure and understandings of the officer group. Both these new categories of officers provided a significant challenge to the power relations and informal systems of hierarchy that were already being taken apart and restructured.

\section{Fighting The Changes}

Employee dissatisfaction was predictable following the State Sector Act 1988. Not only did this herald changes such as affirmative action recruitment, but it also had a direct and immediate impact on state employee pay conditions (Walsh, 1994). The first effect was the removal of the annual general adjustment that had come to be recognised by employees as a natural right and mechanism for inflation adjustments. Shortly thereafter, the government legislated to end the Government Superannuation Fund whereby not only were immediate conditions of employment affected but so too were future retirement earnings. The Department of Justice (1990) pamphlet then alerted officers to the foreseeable loss of subsidised housing, and that their prospects of promotion had diminished with the end of the rank structure.

Unsurprisingly, prison officers felt aggrieved that their job was becoming more difficult with less rewards. They had to accommodate new modes of working, destruction of their homogenous group, imposed changes to working relationships and increasingly problematic inmates with seemingly more rights. Inmates were becoming younger, more ethnically diverse and had more violent offence histories. There were increasing numbers of gang members and gang affiliates, and increasing numbers of inmates with psychological problems as a result of the paring down of mental health services (see Department of Corrections, 1999c; Newbold, 1989) - another feature of economic restructuring. Furthermore, inmates were starting to gain a public and official voice. Official complaints and litigious claims made by inmates regarding specific incidents or the system as a whole were beginning to be made on a regular 
basis. Complaints led to inquiries at Invercargill ${ }^{73}$, Mangaroa ${ }^{74}$ and Wellington ${ }^{75}$ prisons in relation to prison officer conduct which previously would have been unthinkable. The most serious of the incidents—at Mangaroa-led to twelve officers being dismissed for improper practices and assault. Furthermore, inmates' ability to lodge more complaints was seemingly encouraged with the introduction of a freephone number direct to the Office of the Ombudsman ${ }^{76}$ (Department of Corrections, 1997k).

The officers' immediate working conditions were also deteriorating as a result of prison overcrowding. As an example, in the early 1990s the target facility initiated double cell provisions by positioning bunks atop existing cell beds. This meant that instead of 56 inmates within an Alpha Jail unit there could be up to 112. The unit in which this occurred was colloquially referred to as The Bronx due to the cramped and squalid conditions which then followed. The practice was terminated when it was recognised that such conditions were in breach of the United Nations convention on minimum prison standards. Nonetheless, additional huts placed within the perimeter fencing of existing units eased the problem, even if reducing the overall space in each unit.

At the same time, the officers’ career structure was flattened, removing any possibility of legitimate formal status and demolishing any remaining motivation to work hard or achieve within the new qualifications framework and imposed professionalism. In addition, the real benefits of state employment, such as job security, perks and

\footnotetext{
${ }^{73}$ See New Zealand Penal Inspectorate (1990).

${ }^{74}$ See Kitchen (1995), Logan (1993) and Unauthored (1993a).

75 See Kay (1993a), Raea (1993) and Unauthored (1993b).

${ }^{76}$ As a result of the incidents at Mangaroa Prison, the Ombudsmen's role within the prisons increased dramatically (Department of Corrections, 1998a). From 1995 the Office of the Ombudsmen employed three investigating officers who regularly visit each prison, investigate complaints by inmates and debrief management regarding issues that arise. As an example of the workload of the office, inmates raised 1726 complaints in the 1996/ 1997 reporting year (idem). Staff were not afforded the opportunity of such access for their grievances. Formally they could contact the Ombudsmen, as this office was not limited to prison complaints and thus not limited to inmates. However, in discussions with staff it was clear that they were weary of the motivations of this office and hence reluctant to raise issues beyond responses to investigations. In 1994 a telephone number was provided as an 'incident line' for staff to report major incidents (Department of Corrections, 2000b). This line was for the coordinated management of incidents to allow officers to deal with the matter while Head Office communications prepared media statements and contacted the appropriate Managers and Ministers. The perception from officers was that this system was primarily for face management, so the majority of incidents were dealt with locally, reported via incident reports or reported later through IOMS.
} 
privileges were becoming a thing of the past. Militancy amongst the prison officers increased as a result and led them into industrial action. The proposed reduction, and in some cases elimination, of penal rates in 1991 proved to be the beginning of this. Although the loss of penal rates had already occurred across the public sector, prison officers were particularly affected by this prospect as the essentially closed environment of the prison had allowed them to secure considerably more perks and privileges through the manipulation of their previous conditions of employment than most other state employees (Department of Justice, 1988a). Strike action was issued by the PSA in 1991 but did not eventuate. After six months of contentious and protracted negotiations, a transitional collective employment contract was signed. Negotiations continued to be strained between the two parties, particularly in regard to negotiating out penal rates. Prison officers were disappointed that their union had conceded without strike action at this time.

In mid-1993 the army was put on standby to take over the running of the prisons as tensions mounted again between officers and management (Ambler, 1993a). Prison officers had stopped accepting inmate receptions on PSA advice. As muster capacities were reached, the police were placed on alert because the overflow of inmates was required to be accommodated in their cells (Vasil, 1993a). It was reported that tension was so high that inmates were threatening to riot at the prospect of army involvement (ibid, 1993b). The PSA issued a ban on overtime with the threat of a ban on inmate transfers to follow (Ambler, 1993b; Kay, 1993b). At this juncture, resolution was reached with a one off payment of $\$ 6000^{77}$ per officer in exchange for removing security allowances and penal time payments, and a rise of $30 \%$ to base salary for existing employees (Kay, 1993c). All displaced ranking staff were placed in a newly created salary grade ${ }^{78}$ (Sarr, 1995).

\footnotetext{
${ }^{77}$ Officers that were receiving a travel allowance were permitted to retain this for a reduced payout of $\$ 5000$ or could accept the $\$ 6000$ and cease receiving the travel allowance. The PSA had argued to maintain this allowance as there was a reluctance from prison officers to use public transport whilst in uniform as they reported receiving abuse from ex-inmates (Vasil, 1993b).

${ }^{78}$ The new 'grade' was contentious as these officers received a mere \$300 per annum less than Unit Managers. Imbalances in remuneration had been a problem for some time. For example, the Ministerial Committee (1989) noted that in the financial year of 1987/ 1988 some prison officers, third officers and second officers, had annual incomes exceeding that of the highest paid superintendent and many more had an income exceeding that of the two lowest graded superintendents. At the time of my research, several senior officers that one would have expected to be interested in the 2IC or Unit Manager roles expressed to me that there was no financial advantage to the additional responsibilities. Indeed, for
} 
The manner in which the PSA had conducted the 1993 negotiations left many officers disillusioned and dissatisfied. There was a feeling that it was not recognising the supposed uniqueness of the officer group from the other state employees whom the PSA represented, thereby diluting their overall conditions rather than advancing them (Vasil, 1995a). Furthermore, upon reconsideration of the deal the PSA had negotiated, officers realised that even with the $30 \%$ increase in base salary, many of them were significantly worse off financially due to the loss of penal rates which for many had supplemented their take-home pay by up to $100 \%$. As a consequence, more than 900 officers withdrew from the PSA and formed the POA ${ }^{79}$ (idem). The POA issued a statement in which they said they were adamant that many prison officers were not satisfied with the arrangements negotiated by the PSA (Unauthored, 1993c). Indeed it was reported that more than one third of staff votes were against accepting the 1993 deal (Vasil, 1993c). The POA grew in popularity with 1300 to the PSA's 800 members by the time of the 1996 negotiations regarding opposition to officers' tasks being subcontracted out to casual workers (Unauthored, 1996a).

Within weeks of concluding the 1993 industrial crisis, the government announced the provisions for private consortia to tender for finance, design, construction and management of two prisons within the Auckland regions (Unauthored, 1993d). What had hitherto been a passive threat of privatisation now appeared to be a likelihood. Indeed, the threat of privatisation frequently hung over subsequent industrial relations (see for example Gardiner, 1994; Goff, 1994; Kilroy, 1994; Unauthored, 1994a, 1994b, 1995). To illustrate this point, during the 1994 pay negotiations the government's plan to contract prison management was formally placed on the agenda by the Department of Justice (Unauthored, 1994c). To further aggravate the tensions between the prison officer staff and the Department of Justice, at the same time as the pay negotiations in 1994 it was announced that Justice staff, excluding prison officers, would receive a $\$ 1000$ bonus payout as a result of an underspent budget and in recognition of their hard work in the preceding twelve months ${ }^{80}$ (Vasil, 1993d). The

some it would mean surrendering certain historically gained allowances and thereby a decrease in overall salary.

${ }^{79}$ Note that the POA was referred to as the 'Prison Officers Association' and 'Penal Officers Association' depending on the documentation.

${ }^{80}$ This was particularly aggrieving to prison officers as the Department had reported a lack of funding in the budget as the rationale for their failed pay rise bid. In reality, this was likely the case as prisons 
prison officers' negotiations had resulted in no pay increment, nor had they had one for four years (ibid, 1993b, 1993c).

It seemed that for officers, deteriorating working conditions, imposed changes in working practices and recruitment and deteriorating pay had all converged. In the light of this, industrial action seemed to be the officers' only recourse. However, even this seemed to be ineffectual. For example, an impromptu stop-work occurred at Wanganui Prison when officers walked off a night watch shift protesting at low staff levels (Unauthored, 1994d). The consequence, however, was a formal disciplinary investigation, although no officer was formally disciplined. No attempt was made to address the conditions that had led to the stop-work protest. Methods of bringing attention to issues had to change. When Mount Eden Prison prison officers wished to challenge their administration, aware of the ineffectiveness of the Wanganui Prison walk-off, they took their concerns to the Employment Court asserting that they were unable to work effectively or safely as muster levels had exceeded the maximum and staff levels were insufficient (ibid, 1997a). Although the result of this action is not known, it shows the move away from traditional militant responses involving withdrawal of labour to the opening of more litigious channels involving financial redress. Interestingly, the only successful case of compensation offered to an employee still employed by the employer in the New Zealand Employment Courts involved payment damages of $\$ 22,250$ to a prison officer from the Department of Justice for their handling of an assault investigation against him (Kay, 1994).

The litigious approach was also invoked in response to the recommendation of the Ministerial Committee (1989, p. 84) that "the provision of Departmental prison housing and villages be phased out”. Officers at Manawatu Prison sought an injunction of their eviction as a breach of their employment contract which provided occupancy rights (Unauthored, 1994e). Judge Goddard, cited in Vasil (1995b, p. 1), ruled in favour of the prison officers stating "[w]hat [wa]s involved [wa]s a confiscation of the plaintiff's rights of occupation without any offer of compensation ... a classic and obvious abuse of power”. While this delayed the process, the Department continued to 
negotiate away the housing provision. Eviction notices at the target institution were issued in 1997.

However, notwithstanding legal recourse by individuals or groups, the majority of contract negotiations were still conducted by the unions. The 1994 contract negotiations received a divided response from the unions with the POA willing to accept the \$1000 salary increase while the PSA instructed their members to reject it (Vasil, 1995a). Dissention between the two unions again arose in relation to 1996 pay negotiations. This time, however, it was the PSA which accepted the \$1250 increase while the POA opted for industrial action. At this juncture, the POA was particularly concerned with the proposal to use casual staff on officers' preferred tasks of escort and hospital guard duties ${ }^{81}$ (Bain \& Daniels, 1996; MacKenzie, 1996a; Unauthored, 1996a). As the strike loomed, division became rife within the service. Several prison managers approached POA union members offering them individual contracts (MacKenzie, 1996b). Some officers took this option, while a further 289 officers nationally switched allegiances to the PSA and accepted the negotiated contract on the first day of the strike. A further 38 officers similarly transferred to the PSA during the fifteen days of industrial action (MacKenzie, 1996a, 1996c; Unauthored, 1996b). POA members who were 'making the sacrifice of pay for the perceived greater good of the group' were furious.

PSA officers were also under pressure at this time. Defectors were being subjected to verbal abuse, physical assault and damage to cars as they crossed the picket lines (Bell, 1996). The Department of Corrections issued notices threatening PSA members with dismissal if they refused to work overtime and return to shift on their days off to cover the staff shortfall ${ }^{82}$ (Vasil, 1996). Then, after fifteen days of strike, 86\% of POA members voted to return to work, predominantly due to the financial hardship they and their families were feeling heading towards Christmas (MacKenzie, 1996d). The only concession won was the inclusion of the proposed casual workers under the POA collective agreement. The previously united prison officer group had, as a result,

\footnotetext{
${ }^{81}$ Concerns regarding the use of casual staff were again ignited when complaints were made that an inmate had access to sex, drugs and alcohol during a hospital stay under the escort of a non-prison officer (see for example Unauthored, 1997b, 1997c).

${ }^{82}$ Much of the staff shortfall was covered by the use of military personnel again, however, the Department of Corrections endeavoured to have a reasonable proportion of prison officer staff per shift to ensure routines were minimally disrupted.
} 
become bitterly divided when my research began in $1997^{83}$. For example, on the list of 'things to know' which officers conducted me through was an indication of those who had not supported the 1996 strikes; or worse, those who had defected. Indeed many POA members still wore their official strike pins on their uniform as an overt display of their hostility at those who had betrayed their allegiances to the 'authentic' prison officer group ${ }^{84}$.

By 1996 prison officers’ salaries ranged from $\$ 29,000$ to $\$ 45,000$ with the average at $\$ 42,000$ including penal rates but excluding overtime (ibid, 1996e). This was significantly lower than take-home pay in the early 1990s, which was regularly supplemented by generous penal rates, double shift bonuses and so on. Officers reported to me that it was not uncommon, in the past, to informally negotiate 'shift swaps' to manipulate penal rates to achieve higher take-home pays. In such ways, end of year salary figures were at least twice what they had become in 1996. It was these 'abuses' of penal rates which the Department of Justice had targeted in the 1993 negotiations.

Contract negotiations continued to be difficult, although after the disillusionment of the 1996 strikes, officers were reluctant to allow industrial action to impact on their weekly pay packets again ${ }^{85}$. During the 1997 contract negotiations, the PSA and POA organised their respective approaches and agreed to support one another in the event of any industrial action ${ }^{86}$. There has not, however, been a strike since 1996. It had become clear to officers that their unions were not the protective force they once may

\footnotetext{
${ }^{83}$ POA members were considered more militant as they were generally more dogmatic in their views and many were the original defectors from the PSA. For the purposes of my research, officers were not directly questioned as to their allegiance of union as it was not perceived beneficial to raise any antagonism on this issue that may have been misdirected at the research purpose. However, informal notes of allegiance, as expressed by various staff members, revealed that the POA group was typically the four years and beyond service length officers. That is, those that had experiential history in the early to mid-1990s fraught negotiations.

${ }^{84}$ A similar symbolic division was noted at Attica Prison in USA whereby strikers wore tee-shirts and jackets proclaiming 'we walked the line in ' 79 ' as a hostile affront to non-striking officers, a hostility and division that similarly remained for years (Zimmer \& Jacobs, 1981).

${ }^{85}$ Indeed, issues that had previously created significant reaction by the unions passed by into effect with little overt opposition. For example, prisoner escort and court custodial services were opened to public tender in March of 1998 with a successful contract negotiated with an outside provider by July (see Department of Corrections, 1998c, 1998d).

${ }^{86}$ In 1999, in line with the emphasis on the language of correctionalism within the service, the POA reformed as the Corrections Association of New Zealand (CANZ; CANZ, 2005) with additional strength for its members as it obtained affiliation with the New Zealand Council of Trade Unions (NZCTU; NZCTU, 2005).
} 
have been, and that the loyalty and support of the officer group could no longer be relied upon.

\section{Facing Up To Change In The Aftermath Of Defeat}

A review by Newbold and Eskridge (1994) found the overall effect of $\mathrm{He}$ Ara Hou had been positive. They reported: a declining escape rate; decreased inmate suicide rates relative to external society rates; fewer serious assaults on staff; and an overall increase in morale ${ }^{87}$. They highlighted: the erosion of formal barriers between inmates and staff with the increased usage of first names by both groups; staff and inmate combined efforts in cabaret, dramas and other performances for public consumption; and joint participation of inmates and officers in sporting teams for external competitions (idem). However, in the late 1990s, such positive attributions, if present at the start, had quickly eroded. In 1997, for example, there were: two separate riots ${ }^{88}$; a hostage taking ${ }^{89}$; organised protests by inmates ${ }^{90}$; high drama escapes ${ }^{91}$; and a series of serious assaults on staff ${ }^{92}$. Whatever positive changes the reforms had produced, these had also been accompanied by turbulence and disorder.

Previously, stability within the prison had been provided by straightforward and unchallengeable power arrangements. There had been disturbances, but the status quo was quickly restored as historical social arrangements were understood by both officers and inmates. For prison officers, power derived from one's hierarchical

\footnotetext{
${ }^{87}$ The concept of morale was not distinguished as to whether reflecting that of staff, inmates or both.

${ }^{88}$ The first riot was at the target facility (see Bates, 1997; Stutchbury, 1997). The second riot was at Paremoremo Prison where there was a standoff between intoxicated armed inmates and about 70 officers (Unauthored, 1997d, 1997e).

${ }^{89}$ Four prison officers were taken hostage during a 26 hour standoff where four convicted murders had wired up supposed gelignite down a wing and around the area the hostages were held (Bates \& Venter, 1997; Unauthored, 1997a, 1997f).

90 Eighty inmates at Christchurch Prison staged a sit-in protest to challenge regime changes (Unauthored, 1997g).

${ }^{91}$ For example, two escaping inmates stabbed three officers at Christchurch Prison (Unauthored, 1996c).

${ }^{92}$ For example, a prison officer required surgery after a stabbing at Paremoremo Prison (Unauthored, 1997h); a Wellington Prison prison officer was hospitalised after a serious assault from an inmate (ibid, 1997i); and the target institution had four serious assaults against prison officers within a month whereby the POA called for immediate action as this prison now ranked as having had the highest number of serious assaults on prison officers within a defined period of time (ibid, 1997j, 1997k).
} 
position within the group. The formal and informal status hierarchies were aligned and based on length of service, with additional status (beyond the rank structure) only achievable through prowess on the 'shopfloor'. In this way, power relations and movement within the status hierarchy were simple, transparent and dependable. The arrangement was highly functional and everyone knew their place. As all officers would progress with time, there was no need to challenge it, and without challenge there was stability.

With regard to social relations, there may have been some in the prison officer group who associated more often together in the past, Goffman's (1961) 'buddy' relations, but it was unthinkable for them to flaunt this association let alone use such relations to challenge the existing hierarchical arrangements. Boundary lines between inmates and officers were similarly transparent and unquestionable. These were two separate groups which co-existed in parallel with each other, both attempting to do their time and secure as many benefits as possible from the system which encased them both. However, within a short period the stage on which both enacted their well-rehearsed scripts was to collapse. The boundaries between inmates and officers were to become uncertain, even contestable by both groups, as they sought to carve out an existence and understanding within the corporatised prison system and new modes of working. This was complicated further by the seemingly wanton destruction of the very feature that had kept the system stable, namely officer rank and the power that came with this.

It is acknowledged that while this set of arrangements may have been useful in maintaining equilibrium, not all officers or inmates experienced it positively. Such rigid unchallengeable social dynamics were highly amenable to abuses of power, such as unquestioned adherence to any orders from superiors, and protection from criticism or discipline through closure of ranks. It was such abuses which the prison administration had intended to eliminate with the removal of the ranking structure. What was problematic, however, was the absence of any alternative means of establishing hierarchy. To remove established power relations while maintaining a system of control required deliberate and concerted manipulation of social dynamics to ensure a new system filled this void. Instead, the social control mechanisms were effectively destroyed and then left, without direction, to re-establish themselves in unanticipated ways. Lines of authority which had been clear in the past had been 
disrupted and the emerging status systems and power mechanisms were generating a fragmented and confused staff. The rapid influx of new staff at a time of turbulence within the prison service increased the potential for destabilisation. All the while, no one, not even their unions, seemed to be able to protect or assist the officer group. 


\section{CHAPTER FOUR: THE SETTING FOR THIS RESEARCH AND THE PEOPLE INVOLVED}

The effects of the structural changes imposed on the prison service were the focus of my research. These were acted out at the target prison which, in itself, because of the changes, provided the setting for two different 'prison plays' to be acted out. Now there were Alpha and Beta Jails instead of just the one prison that there had previously been. Following an examination of the target prison, this chapter will outline the resulting demographic structure as at the time of this research. In many respects my group of officers were similar to the findings of international research. However, they differed in two key ways - length of service and ethnicity — that will be demonstrated further in this thesis to have had profound effects on the officer group.

\section{The Target Prison}

This had been initially designed as a semi-open (minimum security with no perimeter fencing) adult male jail during the 1920s era of prison farm development. The facility was largely self-sufficient, running sheep, cattle, pigs and a market garden. During an overcrowding crisis in the 1950s it was required to convert one of its wings into a borstal to take some of the overflow from elsewhere. While initially a temporary arrangement, the borstal facility remained for over a decade. In 1964, as a result of the upgraded inmate classification procedures, the inaugural 'First Offenders' Classification Unit' was opened. To appropriately accommodate this new function it was decided that the target prison needed full reconstruction. This was completed by 1967. This structure remains in place today and was considered a show prison of its time ${ }^{93}$.

\footnotetext{
${ }^{93}$ Appendix E illustrates the general plan of this original prison (labelled 'Alpha Jail' for the purposes of my research).
} 
Its surrounding scenery is picturesque with the prison nestled into a valley with a mountainous backdrop to one side and a forest hillside to the other on 160 hectares of farm land (Department of Corrections, 1997l). If it was not for today's razor wire and floodlights, one might mistake the facility for an elaborate camping ground. The prison village was in the immediate vicinity, as were the military police grounds. Although the military police were completely distinct from the prison ${ }^{94}$, their presence, and the fact that the prison's land was leased by the Department of Corrections from the Ministry of Defence, meant that the road through the centre of the target prison site was required to remain designated as 'public' and thereby not technically governed by the Penal Institutions Act $1986^{95}$. For this reason, there was not a distinct access sentry prior to entry to the prison grounds or perimeter security around the outer circumference of the institution. The public could freely walk or drive through the car parks and up to the Alpha Jail main door or into the Beta Jail car parks ${ }^{96}$. Inmates working in the Alpha Jail garden areas, market garden area or walking between units had access to the public and staff car parks or, if they continued to walk down the road, to 'freedom' ${ }^{\text {97. }}$

Prison industry varied over the history of the institution. While cultivation of basic produce continued throughout, it had been considerably reduced by the time my research began. The prison had once supplied local stores. However, as this was deemed unfair competition for outside providers, it was required to reduce capacity to supplement prison supplies and some charitable organisations and community groups. Livestock was restricted to sheep for supply of wool in the later part of the 1970s as the facilities and operation did not comply with various acts and regulations, such as

\footnotetext{
${ }^{94}$ At no time were the military police involved in any prison activities, not even during escapes, violent incidents or the riot. The only time one noticed their presence was the intermittent unmarked car passing through the area to the police grounds (around twice a day). It was unlikely inmates were even cognisant of its presence (although I did not ask them to avoid bringing unnecessary attention to it).

95 While officers were aware of this it was also recognised that laypeople were not aware of the limitations of the statutes, therefore, reference to the Act or to the grounds as belonging to the prison tended to have the desired effect of compliance from 'intruders'.

${ }^{96}$ Car parks and roads are marked in solid block black on the schematic images in Appendix E.

${ }^{97}$ Inmates are more likely to 'not return' from an external work gang than to 'escape' while in the vicinity of the prison grounds as it is a significant walk to access public transport. It was well documented that most escapes from New Zealand prisons amounted to little more than non-return from organised leave/ temporary release or work gangs than exciting breakout escapes (see for example Department of Corrections, 1996b, 2004b; Ministerial Committee, 1989).
} 
the Meat Regulations $1969^{98}$. This in turn became too costly to sustain and all livestock farming ceased.

At the time of my research, industry and employment opportunities for inmates varied significantly. These included the usual perfunctory tasks such as: cleaning the wings, administration and guardroom areas; laundry; and the coveted kitchen duty. Other onsite tasks included: manufacture of toys; market gardens; general gardening; an apiary; traditional Maori carving; delivering various goods to the units; and the manufacture of furniture and construction of pre-fabricated huts for new prisons. Inmates, either ex-tradesmen, or with the assistance of trade-skilled officers, were also involved in the majority of the repairs to and maintenance of the prison site ${ }^{99}$.

Prior to the 1990s, Alpha Jail had no security fences because it had been a minimum security institution ${ }^{100}$. The intention was that further wing development could occur. However, this did not eventuate (additional units became Beta Jail). Therefore, Alpha Jail could be described as two parallel units. Each unit was divided in the centre by an association area effectively creating four discernible wings ${ }^{101}$. Initially these four wings were considered distinct. For a period of time during the 1990s, including the majority of the research period, the four wings were effectively run as two units with the centre dividers open. After the riot in 1997 (see p. 87), each unit returned to running two distinct wings, though one set of staff and manager were assigned to each of the two sets of wings - hence they remained two units. One of the units was the regional remand facility; the other was the regional medium security unit. Alpha Jail had one other unit, essentially non-custodial apart from the suicide watch cells ${ }^{102}$. This unit was staffed typically by senior/ experienced officers, and was responsible for: communications (internal and external); general security patrols; management of

\footnotetext{
${ }^{98}$ To fulfil the requirements of the Meat Regulations 1969, and subsequently the Food Act 1981, would have been too costly for the perceived benefits of: rehabilitation of inmates through trade training, this could be achieved in alternate and less expensive trade settings; or meat supply, particularly as the cost could not be offset through the sale of meat (due both to the nature of unfair trade arrangements and the Food Hygiene Regulations 1974 governing the preparation of meat for sale).

${ }^{99}$ External contractors were typically only contracted for direct security issues, such as electronic gate failure, razor wire repair, faulty camera operation and so on.

${ }^{100}$ Security perimeter fences and razor wire were not added until the 1990s.

${ }^{101}$ Note that each unit contained facilities for 56 inmates; 28 in each wing (two sides of 14 cells).

102 At the time of my research there were holding cells located next to the central guardroom and reception area that could be viewed continuously by the officers on duty, hence their use as suicide watch cells. Shortly after the interview phase a separate specialist annex style 'unit' was added to the rear of Alpha Jail that revoked the use of these cells.
} 
inmates' stored property; alarm monitoring for the entire prison (Alpha and Beta Jail units); escorts (such as to hospital, courts, funerals and so on); general reception; receiving inmates (processing in and out of the facility); and 'spare' staff deployed where additional staff might be needed. This was a last resort measure because this unit was equally as short staffed as other units. Nevertheless, it was the first to mobilise in response to an incident alarm.

The physical layout was as follows: cells lining a central enclosed corridor; thick solid metal doors with large locks and small peephole windows; solid impermeable brick walls; reinforced steel grills dividing areas and covering windows; poor ventilation and pungent air thick with lingering decade old smells of cigarette smoke, poor hygiene and food preparation. When inmates were out of their cells and generally milling around the wing, there was very little room for them to manoeuvre without encroaching on each other's personal space. Inmates' association time was typically spent in the wing. Some reprieve could be offered in an adjacent gymnasium building or in fenced or fully caged (depending on the inmate's security classification) ${ }^{103}$ yards between the units.

During the 1980s, prison design changed considerably and the Beta Jail units reflected this (see Appendix E to compare the two jail designs). Virtually every unit was geographically and functionally distinct. The exceptions were: two units which were surrounded by the same razor wire perimeter fence as there was a sports field inbetween (access across the field to the adjacent unit still required a security key and passage through two gates so they were still 'distinct'); and two units which were physically connected as they shared a dividing perimeter fence. The latter two units shared kitchen facilities, but were in all other respects functionally distinct.

The physical layout of Beta Jail units was far more congenial, reflective of the tabloid press 'holiday camp' imagery. The external perimeter fence was mesh with a razorwire top but barely visible from within the unit. Entry was through a controlled sentry box type gateway, operated either remotely from the guardroom under the watchful

\footnotetext{
${ }^{103}$ The yards for the remand block were wire mesh; one was fully enclosed (including a mesh roof) while the other two had open roofs. These yards also had an overhead sentry box and catwalk for officers to observe from. The yards for the medium security unit were much larger and merely fenced (largely by the unit walls).
} 
eye of cameras or manually by a unit duty officer. Once inside though, the building materials were unimpressive: standard hollow wood doors ${ }^{104}$; fibreboard clad walls and plain shatter resistant glass in unobstructed large windows ${ }^{105}$. While each Beta Jail unit had unique features, the essential design was three perpendicular rows of twenty cells surrounding a central grassed recreation area with an administrative block at one end completing the 'enclosure'. All Beta Jail units were minimum security and enjoyed reasonably long unlock periods and association times, certainly later than Alpha Jail units.

Just as every Alpha Jail unit had a formal label/ function, every Beta Jail unit similarly had an assigned function, albeit some designations were informally attained. All six Beta Jail units were minimum security, one was an Identified Drug Users Unit $^{106}$, one a Violence Prevention Unit ${ }^{107}$ and two were Harmony Units ${ }^{108}$. The remaining two units were known in prison parlance as the Work Unit ${ }^{109}$ and the 'Bro'

\footnotetext{
${ }^{104}$ Each cell door had standard home security style locks. In some Beta Jail units inmates had their own key for their cell door which they wore on string around their neck. Additional external door locks were used at night for lockdown but essentially during the day inmates could maintain security over their own space and possessions.

${ }^{105}$ There were no grills on these windows and the only requirement with the curtain was for there to be at least some visibility into the cell at night for musters. There were secure-stays that prevented the window opening wide enough for an average person to climb through (around 80 kilograms; there were many tales of smaller inmates climbing through these at night and clambering onto the roof-tops for drug drops or simply the excitement of breaking the rules).

${ }^{106}$ The label Identified Drug Users Unit was a misnomer as while the intention was there to initiate specific services in relation to such identified inmates, during the course of the research period no such services were implemented, nor were inmates selected for this unit with any other criteria than having minimum security status.

107 The Violence Prevention Unit was atypical in the New Zealand design in regards to routines, management and staffing. Therefore, this unit was not included in my research. Only around half the staff of this unit were custodial. The remainder were predominantly psychological service providers, rehabilitation workers and so on. The inmates within this unit were involved in an intensive pre-release programme to address their identified criminogenic needs associated with their violent offending. For elaboration on this unit and Violence Prevention Programme, see Department of Corrections (2004c).

${ }^{108}$ Harmony Units were for specifically selected mainstream inmates that were required to sign an obedience bond or contract stating their intention to behave whilst in the unit. The implicit threat was to return to the more restrictive Alpha Jail if they breached this 'contract'. This enabled the mainstreaming of inmates that would otherwise have required segregation for their own security and safety, such as convicted child abusers. It was not a segregation unit, although many of its inmates would otherwise be so labelled. The initiative was started in 1991 at Auckland Prison's West Division where an inmate committee elected to have 'harmony bonds' in return for greater privileges and freedoms (Newbold \& Eskridge, 1994).

${ }^{109}$ The Work Unit was so designated as it had the largest proportion of inmates that were assigned to external work gangs (physically left the prison grounds) and greatest proportion of prison officer staff designated as gang officers.
} 
Unit ${ }^{110}$. Since the completion of the data collection phase, several new units have opened including: a Faith-Based Unit, Maori-Focus Unit and Youth Unit.

Table 4.1 provides an overview of the general job designations of the total staff at the target institution at the time of my research. It can be seen that prison officers constituted $77.8 \%$ of the total staff group. The target institution thus had a different staff division to that documented by Liebling and Price (2001, p. 14) for the English and Welsh prison staffs. In that study only $55.5 \%$ of staffs were prison officers ${ }^{111}$. The numerical dominance of the prison officers at this New Zealand prison had a significant effect on their own perceived importance. They were the staff set and felt that they should be recognised as such.

\begin{tabular}{|c|c|c|}
\hline \multicolumn{3}{|c|}{$\begin{array}{l}\text { General Job Designations of the Total Staff Group as at } 11^{\text {th }} \text { Aug } \\
1997 \\
\text { General Job Designations } \\
\begin{array}{c}\text { Number and Proportion of Total } \\
\text { Staff Group Within Each Job } \\
\text { Designation }(\mathbf{N}=\mathbf{2 0 3})\end{array}\end{array}$} \\
\hline Extended Leave & (10) & $4.9 \%$ \\
\hline Ancillary White-Collar & (14) & $6.9 \%$ \\
\hline Ancillary Blue-Collar & (9) & $4.4 \%$ \\
\hline Unit Managers & (8) & $3.9 \%$ \\
\hline Upper Management & (5) & $2.5 \%$ \\
\hline Prison Officers & (158) & $77.8 \%$ \\
\hline
\end{tabular}

Note. Percentages rounded, total may not equal $100 \%$.

Notwithstanding their own self-image, competition and contestability was beginning to develop between the officers in the two jails. As such, the geographical separation of Alpha and Beta Jails supported a natural division into two distinct sets. While this in itself would not necessarily fracture the unity and solidarity of prison officers, it did

\footnotetext{
${ }^{110}$ This unit was labelled as such in reference to the clique that had developed within it called the 'Bros'. These staff members were typically 'brown' skinned, hence the 'Bro' ascription (a shortening of the colloquial 'brother' label typically applied by dark skinned males referring to other dark skinned males). This clique will be discussed further in chapter seven.

${ }^{111}$ Liebling and Price (2001, p. 14) reported only 55.5\% of staffs were officer grades, $2.4 \%$ were governor grades and $42.1 \%$ were the equivalent of the ancillary white-collar and blue-collar staffs shown in Table 5.1.
} 
help to achieve this, given the emerging ethnic and service length features of the New Zealand prison officers.

\section{The Prison Officers And Their Backgrounds}

At the time of my research, the characteristics of the officers at this institution had begun to diverge sharply from those to be found in most international literature on this subject. According to this body of knowledge, prison officers are:

(i) Predominantly male. For example: Cheek and Miller (1983, p. 108) reported that $87 \%$ of their New Jersey correctional officer study were male; Jurik and Halemba (1984, p. 555) reported that $80 \%$ of staff at their research facility, a medium security American jail, were male; Kalimo (1980, p. 52) reported that $86 \%$ of staff participants ${ }^{112}$ in a Finnish Prison were male; Liebling and Price (2001, p. 16) reported that $83 \%$ of the total English and Welsh Prison Officer staffs were male; and Owen (1985, p. 149, 1988, p. 57) reported that the San Quentin guard force was 80\% male. Indeed, Philliber (1987) asserted that women were more underrepresented within male inmate prisons than any other area of the labour force.

(ii) Overwhelmingly white. For example: Cheek and Miller (1983, p. 108) reported that their New Jersey sample of prison officers was $78 \%$ white, $20 \%$ black and $2 \%$ Hispanic; Jacobs (1978, p. 186) reported that 85\% of Illinois prison officers were white; Kauffman (1985, p. 382) reported that for her Massachusetts Prison the ethnic distribution was 90\% white, 3\% Native American and 8\% black; similarly Kauffman reported that the ethnic distribution of Walpole Prison officers was $77 \%$ white, $8 \%$ Native American and 15\% black; and Liebling and Price (2001, p. 18) reported that within the total English and Welsh prison officer staff 95\% were white, 1.7\% were black and 3.3\% were other or non-identified.

\footnotetext{
112 Note that Kalimo (1980) did not differentiate between officer and non-officer personnel when analysing gender. Therefore the proportion of female officers would be lower in reality.
} 
(iii) In their mid-thirties. For example: Cheek and Miller (1983, p. 108) reported that of their New Jersey prison officers 41\% were 19-30 years and 46\% were 31-50 years; Jacobs (1978, p. 186) reported that the Illinois prison officer group comprised of $25 \%$ under thirty years of age and $60 \%$ between thirty to sixty years of age; Jurik and Halemba (1984, p. 556) reported that at their medium security American prison 61.5\% were between 26-45 years; and Liebling and Price (2001, p. 19) found that over $40 \%$ of officers in England and Wales were aged between 30 and 40 years and that over $55 \%$ were under 40 years of age.

(iv) Likely to have a length of service of around 5-7 years. For example: Cheek and Miller (1983, p. 108) reported that their New Jersey sample had 35\% with less than two years service and 65\% with more than two years service; Jacobs (1978, p. 186) reported that of the Illinois prison officer group 16\% had served less than two years, $61 \%$ had served between two and ten years and 23\% had served over ten years; Liebling and Price (2001, p. 21) found that over 50\% of English and Welsh officers had served less than ten years with over 20\% having under five years of experience; Morris and Morris (1963, p. 76) reported that of the officers at Pentonville Prison 40.2\% were between 0-4 years service, 30.8\% were between $4-10$ years and $29.5 \%$ had over 10 years service time; and Myers (1995, p. 142) reported that of Bathurst Gaol custodial staff $42.7 \%$ had $0-4$ years service and $41.9 \%$ had $4-10$ years service, with a median length of six years service for all custodial staff (female staff had less experience than the males on average with a median length of service of two years for female officers). In addition, Bathurst Gaol figures were similar to New South Wales Prisons generally which had median length of service for male prison officers at 4.87 years and 2.48 years for female prison officers in 1993 (idem).

(v) Likely to have low level educational or trade-based qualifications. For example: Cheek and Miller (1983, p. 108) reported that of New Jersey prison officers $35 \%$ had high school level education, 58\% had some college or technical school education and 7\% had a college degree or other graduate work; Jacobs (1978, p. 186) reported that the Illinois prison officer group in 1975 consisted of 39.5\% with no qualifications, 54\% with low level secondary school or equivalent, 5.3\% low level tertiary equivalent and 1.2\% with high level tertiary equivalent; Kauffman (1985, p. 382) reported that $63 \%$ of Massachusetts prison officer recruits had high school 
education, 23\% had some college and 15\% were college graduates; Myers (1995, p. 147) reported that of the Bathurst Gaol prison officers $7.4 \%$ had no education, $40.7 \%$ had intermediate/ school certificate and 51.9\% had TAFE/ or other low level tertiary study; and van Groningen (1981, p. 43) in a Victorian prison officer study found that $55 \%$ had no secondary school qualifications, $28 \%$ had low to high secondary education and $12 \%$ had some tertiary education.

(vi) Likely to have blue-collar and unskilled backgrounds. For example: Kauffman (1985, p. 383) reported that 74\% of Massachusetts and 70\% of Walpole prison officer recruits were previously employed in blue-collar occupations; Lombardo (1981, p. 188) reported that a large proportion of his sample of Auburn officers, 45.2\%, were ex-factory workers; Myers (1995, p. 145) reported that 60.3\% of Bathurst Gaol prison officers were unskilled or had previously had trade based occupations with a further 20.5\% from low level clerical, sales or service industries; and Webster et al. (1983) in a comparative study of prison officers and the general population reported that prison officers prior occupations were disproportionately in the manufacturing industries, transport and construction industries, wholesale and retail trade and the public sector.

(vii) May have military backgrounds. For example: Fitzgerald and Sim (1982, p. 121) reported that in 1970 at Strangeways Prison nearly $80 \%$ of officers had some form of prior military training ${ }^{113}$; Jurik and Halemba (1984, p. 556) identified that $46.7 \%$ of respondent officers were ex-military; Kauffman (1985, p. 382) reported that 31\% of Massachusetts Prison's and 38\% of Walpole Prison's new recruit officers had prior military experience; and Morris and Morris (1963, p. 76) reported that $28.8 \%$ of interviewed prison officers at Pentonville had entered the prison service direct from the forces.

With these characteristics in mind, Table 4.2 demonstrates the basic demographic categorisations of the total target prison officer group in the New Zealand prison where I conducted my research as at the start and end of the research period. It can be seen that while the overall numbers of prison officers at the target institution

\footnotetext{
${ }^{113}$ It should be noted that this figure represents a national policy aberration whereby the English compulsory National Service has inflated the data. New Zealand's history has never included National Service.
} 
decreased by $5.7 \%$ over the period of study, the overall proportion within each demographic category remained relatively stable. There were around four males to each female officer; around three Pakeha to two non-Pakeha officers; and 85\% of officers had less than 10 years experience. Of the officers that left during the research period, disproportionate numbers were: women, with an attrition rate of $10.7 \%$ compared to the men's attrition rate of 4.6\%; and officers in the 4-10 year length of service category, with an attrition rate of $8.2 \%$ compared to $4.8 \%$ for $0-4$ years length of service category.

Table $4.2^{114}$

Demographic Details for the Total Prisons' Prison Officer Group Population as at the Start (mid 1997) and End (late 1998) of the Research Period

\begin{tabular}{|c|c|c|c|c|}
\hline \multirow{2}{*}{$\begin{array}{l}\begin{array}{l}\text { Demographic } \\
\text { Category }\end{array} \\
\text { Males }\end{array}$} & \multicolumn{2}{|c|}{$\begin{array}{l}\text { Total Target Prison } \\
\text { [START] (N=158) }\end{array}$} & \multicolumn{2}{|c|}{$\begin{array}{l}\text { Total Target Prison } \\
\text { [END] }(\mathrm{N}=149)\end{array}$} \\
\hline & (130) & $82 \%$ & (124) & $83 \%$ \\
\hline Females & (28) & $18 \%$ & (25) & $17 \%$ \\
\hline Pakeha & (92) & $58 \%$ & (86) & $58 \%$ \\
\hline Maori & (52) & $33 \%$ & (49) & $33 \%$ \\
\hline Pacific Island & (14) & $9 \%$ & (14) & $9 \%$ \\
\hline $0-4$ years service & (62) & $39 \%$ & (59) & $40 \%$ \\
\hline $4-10$ years service & (73) & $46 \%$ & (67) & $45 \%$ \\
\hline $10+$ years service & (23) & $15 \%$ & (23) & $15 \%$ \\
\hline
\end{tabular}

Note. Percentages rounded, totals may not equal $100 \%$.

Table 4.3 provides the demographic distribution of the total prison as identified by the questionnaire.

\footnotetext{
${ }^{114}$ The quantitative data within this chapter derives, with the exception of Tables 4.2 and 4.4, from the census style questionnaire distributed across the target institution. The data for Tables 4.2 and 4.4 was derived from official records supplied by the target prison administration throughout the course of the research. As the questionnaire did not have a $100 \%$ return rate the questionnaire figures differ slightly to those presented within these two tables. For the purposes of the discussion the questionnaire data provided the greatest detailed account and was supplemented by more information than official records allowed.
} 
Each Day is Different: Prison Officers and Their Work

Table 4.3

Total Prison: Demonstrating the Proportion of the Total Prison Within Each Demographic Category

\begin{tabular}{|c|c|c|c|}
\hline \multicolumn{2}{|c|}{ DEMOGRAPHIC CATEGORY } & \multicolumn{2}{|c|}{$\begin{array}{l}\text { TOTAL } \\
\text { PRISON }\end{array}$} \\
\hline JAIL TYPE (N=115) & $\begin{array}{l}\text { Alpha Jail } \\
\text { Beta Jail }\end{array}$ & $\begin{array}{l}(45) \\
(70)\end{array}$ & $\begin{array}{l}39.1 \% \\
60.9 \%\end{array}$ \\
\hline UNIT LOCATION (N=115) & $\begin{array}{l}\text { Yellow } \\
\text { Orange } \\
\text { Green } \\
\text { Pink } \\
\text { Lime } \\
\text { Grey } \\
\text { Purple } \\
\text { Excluded }\end{array}$ & $\begin{array}{l}(15) \\
(16) \\
(14) \\
(12) \\
(17) \\
(14) \\
(14) \\
(13)\end{array}$ & $\begin{array}{l}13.0 \% \\
13.9 \% \\
12.2 \% \\
10.4 \% \\
14.8 \% \\
12.2 \% \\
12.2 \% \\
11.3 \%\end{array}$ \\
\hline GENDER (N=115) & $\begin{array}{l}\text { Male } \\
\text { Female }\end{array}$ & $\begin{array}{l}(97) \\
(18)\end{array}$ & $\begin{array}{l}84.3 \% \\
15.7 \%\end{array}$ \\
\hline ETHNICITY (N=115) & $\begin{array}{l}\text { Pakeha } \\
\text { Maori } \\
\text { Pacific Island }\end{array}$ & $\begin{array}{l}(72) \\
(32) \\
(11)\end{array}$ & $\begin{array}{l}62.6 \% \\
27.8 \% \\
9.6 \%\end{array}$ \\
\hline SERVICE LENGTH (N=115) & $\begin{array}{l}0-4 \text { years } \\
4-10 \text { years } \\
10+\text { years }\end{array}$ & $\begin{array}{l}(53) \\
(42) \\
(20)\end{array}$ & $\begin{array}{l}46.1 \% \\
36.5 \% \\
17.4 \%\end{array}$ \\
\hline AGE GROUP (N=115) & $\begin{array}{l}20-24 \text { years } \\
25-29 \text { years } \\
30-34 \text { years } \\
35-39 \text { years } \\
40-44 \text { years } \\
45-49 \text { years } \\
50-54 \text { years }\end{array}$ & $\begin{array}{r}(2) \\
(11) \\
(26) \\
(27) \\
(25) \\
(18) \\
(6)\end{array}$ & $\begin{array}{l}1.7 \% \\
9.6 \% \\
22.6 \% \\
23.5 \% \\
21.7 \% \\
15.7 \% \\
5.2 \%\end{array}$ \\
\hline EDUCATION LEVEL (N=115) & $\begin{array}{l}\text { None } \\
\text { Low Secondary } \\
\text { High Secondary } \\
\text { Low Tertiary } \\
\text { High Tertiary }\end{array}$ & $\begin{array}{r}(17) \\
(29) \\
(15) \\
(49) \\
(5)\end{array}$ & $\begin{array}{l}14.8 \% \\
25.2 \% \\
13.0 \% \\
42.6 \% \\
4.3 \%\end{array}$ \\
\hline JOB TITLE (N=114) & $\begin{array}{l}\text { Probationary Officer } \\
\text { Prison Officer } \\
\text { Instructor Officer } \\
\text { Unit IC } \\
\text { Unit Manager }\end{array}$ & $\begin{array}{r}(25) \\
(39) \\
(16) \\
(28) \\
(6)\end{array}$ & $\begin{array}{l}21.9 \% \\
34.2 \% \\
14.0 \% \\
24.6 \% \\
5.3 \%\end{array}$ \\
\hline MILITARY EXPERIENCE (N=114) & $\begin{array}{l}\text { Military Experience } \\
\text { No Experience }\end{array}$ & $\begin{array}{l}(37) \\
(77)\end{array}$ & $\begin{array}{l}32.5 \% \\
67.5 \%\end{array}$ \\
\hline ADDITIONAL JAIL (N=113) & $\begin{array}{l}\text { No Experience } \\
\text { Additional Experience }\end{array}$ & $\begin{array}{l}(79) \\
(34)\end{array}$ & $\begin{array}{l}69.9 \% \\
30.1 \%\end{array}$ \\
\hline
\end{tabular}

Note. Percentages rounded, totals may not equal $100 \%$. 


\section{Similarities Between Prison Officers At The Target Prison And The Findings Of International Research Literature}

\section{Gender.}

The introduction of women to the New Zealand prison system challenged the male status system and cultural tenet of physical prowess which was associated with it. For some time, male officers vehemently opposed women as officers and instigated calculated 'tests of character' in the hope of breaking the women's resolve and causing them to leave the service. Notwithstanding the resignations by many women as a result, a number remained stoic and their numbers increased steadily to around $17 \%$ of the officer complement. This was in line with other male penal institutions in New Zealand ${ }^{115}$ and internationally, although there were rather more women in the non-Pakeha categories which will be shown further in this chapter to have had an effect on women's solidarity.

There was no difference between gender and length of service categories. As women had not previously been employed in men's prisons prior to 1985, it would be expected that there would be no women in the 10+ years service category. However there were two. Both had transferred from women's facilities ${ }^{116}$. Similarly, given only $17.4 \%$ of all officers were in the $10+$ years length of service category, and only $2.28 \%$ of the total officer group had service over 11.6 years, women's longevity relative to men was not affected by the prior restrictions of employment. There was a slight divergence between means of actual service time with women having an average of 5.1 years and men an average of 6.1 years. These figures actually indicate a longer average service time for women when compared with international research findings (see Myers, 1995, p. 142).

Unsurprisingly, there were greater proportions of males with prior military experience. Unexpectedly, female officers had more experience of additional jails relative to the male officers. This prior experience had usually been in women's prisons since women were generally discouraged from working in male facilities until the mid-1990s. The benefit of having had additional jail experience was the varied

\footnotetext{
115 To compare institutions see Appendix L.

${ }^{116}$ Institutions with female inmates had not restricted employment for men or women as officers.
} 
institutional knowledge and experience that such officers possessed and which all officers recognised as skill and expertise within the prison service. However, this additional institutional knowledge did not translate into additional status for women as their experience was from women's prisons and was thus judged to be irrelevant by the male officers - they had not been working in a 'real' prison. In contrast, being in a men's prison was deemed to be life in a real prison and hence valuable experience. To illustrate, the other officers only gave informal recognition to one female officer for her prior prison experience. She had transferred from Paremoremo Prison, the only maximum security male institution in New Zealand. In this way, a woman's additional experience needed to be exceptional for it to contribute to her standing within the officer group.

\section{Age and job title.}

While official policy placed no restrictions on age range for recruitment or retirement, the vast majority of officers, $67.8 \%$, were between 30-44 years of age. This was broadly in line with the international data. It also indicated a slight increase in the average age of officers since the extensive survey of New Zealand prison officers in 1981 by Roache et al. (1982, p. 30) which identified that 69.8\% were between 25-45 years of age. Nonetheless, in the mid-1990s, most seemed to join the service in their thirties. As expected, the older age groups occupied the Unit IC and Unit Manager job titles with $53.6 \%$ of Unit ICs between $35-44$ years and $50 \%$ of Unit Managers between 40-44 years.

\section{Education level.}

Consistent with the international research literature, the officer group had a low level of educational attainment. Indeed 53\% of officers had nothing more than secondary school education. While $42.6 \%$ of officers had low level tertiary education, which gives the appearance of a reasonable level of educational attainment within the group, $75.7 \%$ of these qualifications were trade certificates and hence practical/ skills based qualifications rather than academic. In this way, $85.2 \%$ of officers could generally be classed as unskilled or semi-skilled. This low level of attainment was further reflected 
within the cited previous occupations of the target officer group with $43.5 \%$ reporting unskilled, 23.5\% semi-skilled and 55.7\% trade/ skilled previous occupations ${ }^{117}$.

There was no difference within gender or ethnicity in educational achievement. On the surface, male officers, particularly Maori males, appeared to have higher educational attainment with high proportions of each group within the low level tertiary category. This was a reflection of the high proportion of ex-tradesmen within these two categories. This was important as academic qualifications produced no visible difference in day-to-day operations and hence were not valued within the officer group. Trade skills, on the other hand, could be readily identified, made a visible contribution to day-to-day operations (such as mending a broken sewer pipe which might take several hours or days before an external trade person arrived) and thus afforded such officers extra recognition as useful members of the officer group. The contrary also applied. As women and Pakeha officers had lower proportions of ex-tradespeople, they were collectively viewed as less useful members of the group. As a result, both Pakeha and women officers tended to be more disenchanted since whatever their aspirations were, they were unlikely to receive either formal recognition from the prison authorities or recognition from their colleagues. They lacked qualifications for formal advancement within the new management structure and seemed unwilling to participate in the informal status structure by using their skills, if they had any, beyond those contracted.

There were expected job title differences. For example, 53.6\% of the Instructor Officer group had low level tertiary qualifications, generally trade certificates which were required for their role. However, there was also an unexpected result $-50 \%$ of Unit Managers only had low level secondary education or no formal qualifications. As far as prison officers were concerned, a largely unqualified management group would not instil motivation to further their education or to progress towards the NZQA industry standards framework. Indeed, there were numerous officers who reported that they had fulfilled prior training requirements, only to have them deemed irrelevant when the ranking structure was removed in the course of the 1990s reforms. Such officers were reluctant to believe that the new requirements would last

${ }^{117}$ For explication of these figures see Appendix P. 
sufficiently long to make it worth increasing their qualifications and certainly placed no value on them increasing their promotion potential. The implicit message to officers was that neither length of service nor educational achievement could advance formal recognition (promotion) within the prison service.

\section{Military experience.}

The proportion of officers at the target institution with military backgrounds was comparable to international data with $32.5 \%$ having prior military experience. However, it seemed that the service was becoming increasingly attractive to such people as $44 \%$ of probationary officers had had military experience. Indeed $62.2 \%$ of those with military experience were within 0-4 years length of service. The high number of ex-military recruits at that time may have been an aberration. Bell (1997) reported that the Defence Force was experiencing high attrition to the prison service following the exposure of the military to prison work during the 1996 strikes. He asserted that the Ministry of Defence could not compete with the relatively favourable conditions of employment offered by the Department of Corrections. While the pay structure was less within the Department of Corrections compared to the Defence Force, conditions of employment such as stability of location, regular rostered hours and so on made the prison service more attractive. This was particularly the case with the army which at this time had increased its rate of unpopular international longservice assignments ${ }^{118}$. Therefore, while senior prison officers were experiencing disillusionment at the reduced benefits of employment and were leaving the service, it seemed they were steadily being replaced by the very type of employee the new policies were supposedly discouraging. As argued by Goffman (1961), employment in a total institution does not constitute a change in moral career development for exmilitary personnel which may account in part for its attractiveness to such workers. Regardless, this was likely to have an effect on the culture that the Department of Corrections were attempting to impose on the prison service.

\footnotetext{
118 The age group of the officers with ex-military backgrounds gave further support to the contention that the prison service offered more favourable conditions relative to the defence force for this group. Their age indicated early retirement from the Defence Force rather than waiting for the traditional completion of 25 years (there is a significant financial advantage for remaining for this period).
} 
Overall, in keeping with the general findings of international research literature, the prison officers were predominantly male, in their mid-thirties, had low level to tradebased qualifications, had blue-collar and unskilled backgrounds and similarly had a reasonably high proportion of ex-military personnel (relative to other occupations). What they valued most in their colleagues was experience of 'real prison work', which thereby automatically devalued women prison officers.

\section{Differences Between Prison Officers At The Target Prison And The Findings Of International Research Literature}

\section{Length of service.}

The average length of service would appear to be similar to the international data as the mean length of service for the total officer group was 5.9 years. However, when analysed further, Unit ICs had an average of 11.2 years service time, Unit Managers an average of 10.4 years, while prison officers had an average of only 4.9 years. Therefore the average length of service for prison officers was in fact lower than that found in the international literature.

Of the total officer group, $46.1 \%$ had $0-4$ years service, $36.5 \%$ had $4-10$ years service and only $17.4 \%$ had more than 10 years service, representing a largely inexperienced staff compared to international research findings (see for example Jacobs, 1978; Liebling \& Price, 2001). These figures would seem to indicate again that the job was becoming less appealing to those with longer service. This was probably what the prison authorities had had in mind when introducing the reforms. In this way, some of the problems of the work culture that were reproduced by long serving officers would be remedied. Officers were aware of this subtle drive and its implications:

\footnotetext{
The bad thing is the Department is trying to get rid of these experienced officers, mainly because they cause a bloody headache because they know too much, you know, they stand their ground. It's like divide and conquer - get rid of the experienced officers and these guys (managers) think they can get what ever they want (from the rest of us).

- Female, 0-4 years service, Alpha Jail ${ }^{119}$.
}

\footnotetext{
119 The officer quotes in this and the following chapters derived from the interviews. As a point of reference for these quotes: grammatical errors have only been corrected slightly to permit understanding, however, on the whole they have been left as authentic as possible (occasional words or
} 
As this extract demonstrates, officers perceived the change initiatives to be a deliberate move by the Department of Corrections to undermine the existing occupational culture and fragment the officer group. However, as Goffman (1959) suggests, and as will be illustrated in more detail later, the likely result of this type of 'divide and conquer' approach would actually be, in time, a decrease in overall attachment and commitment to the organisation and increased engagement in secondary adjustment. For prison officers, the immediate significance was that there were fewer experienced staff to pass on the necessary skills of the job not taught at the prison college (see also Crawley, 2004):

The jail expanded so fast and you had probbies (probationary officers) training probbies, you had new staff training staff, and now we've got $75 \%$ of our staff badly trained, inadequately prepared for the job that they should be doing.

- Male, 4-10 years service, Alpha Jail.

Thus, not only were the necessary skills not passed on, but as indicated in this extract the new officers were 'inadequately prepared for the job’. Goffman (1968) asserted that when a newly stigmatised person joins a group, it is the role of their fellowafflicted to instruct them adequately how to cope with their spoiled identity. Such tuition would adequately prepare them for the job and role they were now expected to fill. As a result of ineffective tutorage at this level, there were increasing proportions of officers who were perfunctory about their approach to their job as they were still oscillating between their stigmatised and normal identification creating less attachment and commitment to the organisation or to their fellow-afflicted. While this means that they were in part insulated from their stigmatised identity, they were also not able to effectively employ the aspects of the occupational culture that would protect them from the adverse effects they were subjected to.

\footnotetext{
phrases that could identify an interviewee have been edited for confidentiality); (pause) indicated a prolonged silence as the officer thought about their next response; swearing and argot usage have been maintained to reflect the speech patterns of the officer group; [...] indicates the non-identifying explanatory term for a directly removed word, typically a noun; (...) indicates an explanatory note or missing word that aids flow, longer explanations may also be provided within footnotes; 'you know' is a phrase used colloquially in New Zealand as punctuation or emphasis of a point, it is generally rhetorical in nature. Importantly, however, within the prison context it was often applied to denote cultural understanding that the listener should be privy to. When speaking to the researcher the officers assumed a high level of 'knowing'; the prison officer descriptors provided after each quote indicate the relevant demographic details to associate with the quote, not all details were listed to preserve confidentiality.
} 
The majority of both Unit ICs and Unit Managers were between 35-44 years of age. This supports the contention that the Department of Corrections had a hidden objective which was to discourage old school officers remaining in the prison service by not encouraging their promotion or demoting them, as in the Unit Managers restructuring round of 1997.

Disestablished Unit Managers were able to retain their existing employment conditions but were required to return to prison officer status with the offer of the Unit IC title. The Unit IC role was predominantly a titular label with regard to formal status (hence being to the side of the status hierarchy on Figure 1.2). There was no limit to the number of officers within a unit who could hold the title. However, only one officer per shift would act in the role. Many of the disestablished Unit Managers subsequently chose not to hold Unit IC status, predominantly because there were no financial incentives ${ }^{120}$. That is, the 'perks' with the Unit IC role were considered insignificant for the additional responsibility. For those who were extremely disillusioned with the process and displacement, a 'work to rule' attitude prevailed. They were no longer willing to provide the additional support and service beyond that which they were contracted to do. Therefore, they regarded not accepting the Unit IC responsibilities as getting revenge on the prison authorities for their loss of power, authority and status.

Even though 'Unit IC' was predominantly a titular label, officers associated the title with length of service and experience. Therefore, an officer with the title, regardless of whether they ever acted in the role, was automatically thought to be 'knowledgeable'. Unit ICs (and Unit Managers) had significantly higher rates of additional jail experience than the three lower level job titles possessing institutional knowledge not available to the majority of the lower job titles. As all officers were aware that the Unit IC role had few formal rewards, and that the Unit IC provided the necessary guidance and expertise for a 'smooth' shift, the officers who took on the additional responsibility were viewed by the remaining officer set as demonstrating

\footnotetext{
${ }^{120}$ The 'perks' associated with the Unit IC role were that they had every third weekend off duty, did not do night watch and were only rostered for the $6 \mathrm{am}-2 \mathrm{pm}, 1 \mathrm{pm}-9 \mathrm{pm}$ or $2 \mathrm{pm}-10 \mathrm{pm}$ shifts. There was no additional special responsibility allowance.
} 
support for the wider officer group ${ }^{121}$. However, the officer group reacted negatively towards staff members of less than four years service who applied and were given the Unit IC position. They were thought to be not experienced enough to be of assistance to the group and therefore could place the other officers on shift in danger. Such appointments provided confirmation to the officer group that upper management were unaware of the characteristics of an effective or efficient officer. This did not occur often, though, as with a lack of incentive to seek 'promotion' to Unit IC, many of the newer officers were reluctant to apply for such a position. Hence, the majority of officers were likely to remain at the prison officer level.

With little prospect of advancement regardless of time served, motivation to excel, to gain additional qualifications or even to remain in the job had become minimal. For those who were at Unit IC level there was no progressive structure either, given the low number of Unit Manager positions and the propensity of the new managerialist ideology to recruit from outside the service. Therefore, as instructor officers held the same rank as prison officers, and Unit IC was titular, it can be seen that after the one year probationary period, $72.8 \%$ of officers remained at this basic level.

Thus, at a time when experience and knowledge would have been most useful for securing stability within the officer group, the influx of new recruits was leading to the reverse. The increasing levels of inexperience within the officer body were to have profound effects on officer group relations and work performance as shall be expanded further in this thesis.

\section{Ethnicity.}

The ethnic composition of officers at the target institution was 62.6\% Pakeha, 27.8\% Maori and 9.6\% Pacific Island. The proportions of non-Pakeha officers was certainly atypical of the ethnic demography of prisons outside of New Zealand ${ }^{122}$ and was disproportionately high relative to the New Zealand general population and working-

\footnotetext{
${ }^{121}$ Disestablished Unit Managers that declined the Unit IC role were not viewed as rejecting the group. Officers were sympathetic to their situation and continued their respect of them as knowledgeable and valued officers.

${ }^{122}$ It is acknowledged that in some international jurisdictions, particularly in USA, there were varied ethnic compositions within different prisons and regions. However, on the whole, the New Zealand general ethnic demography would appear to remain higher than that generally experienced overseas.
} 
age population ${ }^{123}$. To demonstrate that this was a function of the prison service and not 'job type', consider the New Zealand Sworn Police Force ${ }^{124}$. In 1997 they contained significantly different ethnic proportions to the prison officer set with 90.4\% Pakeha, 8.5\% Maori and 1.1\% Pacific Island peoples (New Zealand Police, 1998, p. 105).

Although there had been a significant influx of non-Pakeha officers in the early 1990s, their proportion amongst the new recruits was decreasing. Pakeha were being recruited at a greater rate than non-Pakeha in the mid to late 1990s period despite affirmative action policies to the contrary. For example, Pakeha constituted $88 \%$ of the probationary officers' group at the time of my research. Analysis of ethnic proportions of each length of service category explains how ethnic minorities remained overrepresented within the officer group when their recruitment numbers were declining. For example, in 1997 while 51.4\% of Pakeha officers served less than four years, 62.6\% of Maori and 63.7\% of Pacific Island officers had served over four years. Further, Pakeha had an average length of service of only 5.4 years while Maori and Pacific Island officers both had average service times of 6.9 years. This would suggest most of the non-Pakeha officers were recruited in the early 1990 s $^{125}$ and they were remaining in the job longer. The demographic change as a result of the affirmative action policies during the late 1980s/ early 1990s period was well known to officers:

\footnotetext{
${ }^{123}$ The closest National Census date was 1996 which identified 79.5\% of the total New Zealand population were Pakeha, $14.8 \%$ were Maori and $5.7 \%$ were Pacific Island peoples (Statistics New Zealand, 1999, pp. 137-139). The proportions of each ethnic category in the working-age population for 1996 were 87.4\% Pakeha, 9.0\% Maori and 3.5\% Pacific Island peoples (ibid, 2005, p. 92).

${ }^{124}$ Sworn police staff figures were used in this discussion as they most closely relate to prison officers, whereas non-sworn staffs contain disproportionate numbers of administrative personnel. The figures, however, did not differ much as the non-sworn police force proportions were $91.0 \%$ Pakeha, $6.5 \%$ Maori and 2.5\% Pacific Island peoples (New Zealand Police, 1998, p. 105). The total police staff proportions were 90.6\% Pakeha, 8.0\% Maori and 1.4\% Pacific Island peoples (idem).

${ }_{125}$ The dramatic increase in non-Pakeha recruits in the early 1990s, then subsequent decrease in recruitment rates, can be partially explained by the national unemployment situation. The early 1990s was a period of particularly high unemployment within New Zealand. For example, in 1993, which represented four years prior to the commencement of my research and a separation in my service length categories, there were 217,400 registered job seekers compared to 154,600 in 1997 and 133,800 in 2004 (Statistics New Zealand, 2005, p. 57). In 1991, when the Pakeha unemployment rate was 6.5\%, Maori and Pacific Island peoples were experiencing rates of $21.2 \%$ and $22.7 \%$ respectively (ibid, p. 92). In this way, the appeal of the prison service was compelling for these ethnic minorities. By 1996 unemployment rates had eased somewhat to $15.4 \%$ for Maori and $15.2 \%$ for Pacific Island peoples (idem), hence less compulsion to join the service.
} 
Oh the group is certainly changing, it's changed from a majority of white men... a lot of them were ex-service personnel, to a majority of Polynesians (Maori and Pacific Island peoples) coming in. Staff have had to accommodate that.... [Pacific Island officer's name] was the first Pacific officer to be signed on and that's fourteen odd years (ago), that's not that long. Without a doubt the majority (historically) were British (Pakeha) personnel, not Maori personnel. And now you've got everybody. You've got people who can't even speak English properly... and now you're also moving into the realm of male and female as well! Yeah, we've gone through a few changes in who's hot to hire.

- Pakeha, 10+ years service, Beta Jail.

This extract demonstrates the cynicism that was developing within the officer group, particularly amongst the longer serving Pakeha officers who were beginning to feel excluded when historically they were the dominant group.

While unemployment may well have led many ethnic minorities to join the service in the early 1990s period, there were features within the prison service itself that made this career option somewhat appealing for ethnic minorities and encouraged them to remain in the service. Their high proportion relative to their position in the general population, their noticeable and commanding physical presence and their distinctive in-group ethnic solidarity gave them high informal recognition and value within the prison community. Such stature, the product of their ethnicity, was rare and not usually the norm within New Zealand society as a whole. As a result, Maori and Pacific Island ethnicity began to gain dominance while the previously dominant Pakeha ethos decreased. This cemented the resolve of the ethnic minority groups to stay in the service as they became increasingly more represented in it.

In this way, the restructured New Zealand prison service was beginning to be significantly influenced by minority ethnic culture, an attribute not seen in other Western jurisdictions. In similar societies, the 'white' ethos remained the basis of the prison culture while in New Zealand the numerically dominant group became the outsiders in a prison culture influenced strongly by ethnic traditions.

Overall, the two important differences between the New Zealand target prison officer group and the findings of the international research literature were ethnicity and length of service. The effect of these two variables had the potential to significantly undermine staff loyalties, traditions and cohesion, at precisely the time when those 
values would have been particularly beneficial to the prison officer group, given the erosion of their career structure.

\section{Enforced Cohesion Or Reinforced Differences And Atomisation}

In recognition of the changing composition of the prison officer group, the prison authorities during the early 1990s encouraged the development of 'networks' for minority staff members within the prison in an attempt to enhance their sense of belonging and cohesion. These network groups were intended to provide support and friendship within the prison environment by encouraging the association and socialisation of like-situated officers. As things turned out, the precise groups which the prison authorities targeted were the ones least in need of attention. Network groups involved semi-formalised meetings held at the staff clubrooms at the prison and were open to attendance by any employee of the specified minority. At these meetings staff discussed issues or concerns arising from their employment as well as personal issues. They used the meetings to seek advice from their peers, organise social functions, sporting events and so on. This resulted in a Maori Network, Pacific Island Network and Women's Network being developed at the prison. While well intended, in reality these networks had unanticipated consequences, as discussed below, that further undermined the officer group’s loyalties and cohesion.

\section{The Maori Network}

The Maori Network had existed for several years during the period of high recruitment of Maori officers and had initially provided some degree of cohesion. However, the network disintegrated as the Maori officers formed their own groups and cliques ${ }^{126}$ in the prison. For the cliques, membership and allegiances were

\footnotetext{
${ }^{126}$ The term 'clique' has been used here to denote a particular type of 'group' relations. According to Goffman (1961), cliques consisted of three or more people who exhibited preference for one another's company and exchanged some mutual aid. In this context, cliques were intentional groupings with specific rules of membership, in-group gestures/ language, group behaviours and so on. Cliques are
} 
considered more important than the more general network which had been provided for them. Indeed, the cliques became adrift from other groups of Maori officers and the prison officer group in general. In this way, the cliques, while based around ethnicity, in effect actually weakened the overall cohesion of Maori. Officers were well aware of these divisions:

They (Maori) used to all be one but then they divided into factions cos the other group (the Black Mafia) is a bit too stroppy.

- Pakeha, 4-10 years service, Alpha Jail.

The two cliques which formed had a large influence across the prison, even though only a small number of officers were involved in them ${ }^{127}$. They became a central reference point for all groups in the prison - officers, inmates and management. For officers who were members of the cliques, they represented the last bastion of brotherhood within the officer group; for officers who were not in the cliques, they represented all that was going wrong with the prison service; for management and inmates, they (at least one clique) represented the officers who could reliably prevent disturbances or if needed, re-establish control in times of challenge (typically through informal and often violent means); and ironically (given the majority of members were only in the 4-10 year service category), they were the officers who represented the stereotype of the traditional, unintelligent and at times sadistic prison officer - the very officers whom the Department of Corrections was hoping to replace and yet management were passively supporting.

\section{The Pacific Island Network}

The Pacific Island officers deliberately maintained their network. This was because they had been dispersed throughout the prison and were otherwise atomised. While the network was less formally structured in terms of meetings as the years passed, well supported regular social gatherings and sporting events brought Pacific Island

similar to Sykes' (1958) argot groups. Two dominant cliques existed at the target institution - the Bros and Black Mafia. For more detail on cliques see chapter seven.

${ }^{127}$ Of those who responded to the questionnaire, only $14.9 \%$ where clique members. This figure increased to around $20 \%$ for the entire prison population (based on ethnographic knowledge of nonrespondents). See Appendix $\mathrm{N}$ for further details of clique and group membership. 
officers within the prison, region and even across the country together. In this way, longevity in the prison service and low attrition rate was encouraged by their ethnic identity, camaraderie and group status. They gained status from their bilingual abilities, cultural awareness of Pacific Island inmates' needs and commanding physical presence. This made them particularly effective for maintaining control in their respective units and during inmate infractions throughout the institution.

As these officers were scarce in numbers, it was advantageous to assign at least one to each unit. They consequently became particularly valued members of the prison community. While this status was generally enjoyed, Pacific Island officers were aware that it could have some negative ramifications. For example, their utility might make them indispensable, thus reducing the likelihood that their Unit Manager would approve a transfer to an alternative unit or jail. In practice, this only became possible if a Pacific Island officer who wanted to transfer units informally negotiated with another Pacific Island officer to swap units, jails or even prisons. The problem associated with transfers became well known, not just within the Pacific Island officer group:

I think the only guys that would have problems swapping units would be the Pacific Islanders cos there's only a few in each place and in each unit. If they (Unit Managers) can get their hands on one they want to keep him because they need a translator and they (Pacific Island officers) find it difficult to move around.

- Pakeha, 0-4 years service, Alpha Jail.

It's not all good being Pacific Island... I applied for [non-unit job secondment] and I was turned down because they (management) said I was better suited in the unit with a whole lot of Island inmates. I mean I could have taken it further (Employment Tribunal) but, you know (what's the point).

- Pacific Island, 4-10 years service, Beta Jail.

This lack of ability to move around or gain desired non-unit tasks, coupled with their wide dispersal through the prison, provided an additional incentive for Pacific Island officers to maintain close supportive links within their ethnic group. 


\title{
The Women's Network
}

Officers who had been involved in the Women's Network reported a lack of common interests as a primary reason for its inability to survive long term. It would seem, however, that relying on one's identity as a woman as a basis of unity, distinct from the main officer group or from loyalty to one's unit/ jail, was more central to the groups' demise:

\begin{abstract}
I found them (meetings) a bit of a waste of time. You had the networks to pacify people, not so much to make them think that they had the support group there, but just to make them feel that they're more part of the group. Whereas we should be networking more with the whole group. Like prison officers should be networking together. I guess it was kinda divide and conquer. But I guess you're limited, you know. I can't be supportive of women in [Beta Jail]. I don't know what she's going through. I can only be supportive of women in my jail, but with the networks you got like a probation officer in [Orange Unit] paired with a veteran wing unit officer in [Purple Unit], there's just no connection there. I couldn't see the point in it.
\end{abstract}

- Female, 4-10 years service, Alpha Jail.

Indeed, as this extract indicates, it would seem that the motivations of the Department of Corrections for establishing a separate women's network were interpreted as divisive rather than supportive. It most likely folded after only a couple of meetings because of the effect of gender divisions already existing within the prison culture. This was particularly detrimental to women's status. While the ethnic groups gained status and recognition from their identity, the women's lack of identity led to fragmentation of their support network and further atomised them. However, the women officers tended to blame other women for their subordinate position:

I think I was accepted quite easily... whereas one or two of the (female) officers have been quite staunch and try to act like males and they've had a lot of altercations. Men (officers) don't like to see that and inmates don't like to... be pleasant and you get more out of them (male officers and inmates). I use my femininity to my advantage, so you don't have to be manly. I hate it actually... it doesn't do them (female officers) any good. Two new girls here [two female officers' names] I've seen the way they spoke and a lot of the older guys (senior officers) don't like that, you know. They come across as - I don't know whether it's over compensation or weakness?

- Female, 4-10 years service, Beta Jail.

Some women get quite challenged by other women. They can be worse than men! [Female officer's name] started here and she is immaculate, I think at least some women were quite threatened by her... now a couple of (female) officers 
started running [female officer's name] down and I says 'why?' and then they said that one of the inmates had become infatuated by her... he followed her around like a puppy dog continuously and I said 'yeah, so, did she go into his cell?' and they says 'no he's just following her around' and I says 'so she's doing her job' ... and I says 'I bet you anything that the female staff you've got in that unit are actually threatened by her and are jealous of her cos the inmates says she's pretty'. Bloody female jealousy, we're worse than the men!

- Female, 4-10 years service, Beta Jail.

It was as if highlighting the 'inadequacies' or occupational cultural assumptions of some women (trying to be staunch or being sexually available) would align the criticising women closer to the general male officers' group. In fact, the converse occurred whereby these women merely reinforced the occupational culture rationales for all women's exclusion.

Examining the gendered ethnic division provided some explanation for the divisive situation women found they were in. There were a greater proportion of women in the non-Pakeha categories. This may have created additional divisions within the female officer group. That is, women were identifying first by their ethnicity, then by their gender. This would seem a logical self-enhancing strategy. In the prison setting, more was to be gained from ethnicity than gender. The net effect, however, was a highly divided female group, unable to identify with each other and thereby ineffective at producing a united defence against the various 'pains' that their experience of prison work brought them (see chapter six).

\section{Pakeha Resistance}

The intention of the minority group social networks had been to enhance the morale of those involved. Notwithstanding the fact that, certainly for Maori and women officers, the groups achieved little, their very existence helped to make Pakeha male officers feel that they were the marginalised group within the prison. The network system seemed discriminatory and inequitable to them. To demonstrate their opposition, one Pakeha male officer distributed a pamphlet around the prison advertising the formation and inaugural meeting of an 'Oi-Oi Group'. This was an acronym for 'Officers in [the] Otherwise ignored Group'. The group's name was 
deliberately intended to give offence to the ethnic minority groups because of the association of 'Oi-Oi' as a greeting used by groups such as White Power and other white supremacist gangs. While the Oi-Oi Group initiative was merely supposed to be a reflection of dark prison humour and a display of dissatisfaction with growing Pakeha exclusion, anecdotal evidence suggests that over 50 officers arrived at what had been intended to be a hoax meeting and the original officer needed to be relieved from his shift to facilitate it. This was a clear indication of the real dissatisfaction amongst the male Pakeha officer group at the exclusionary nature of the affirmative action networks.

In effect, across the prison, the previously homogenous prison officer group was beginning to show sharp divisions and mutual antagonisms. Furthermore, the clumsy attempts by the prison authorities to solidify the minority groups had backfired. This had been largely ineffectual anyway, and only succeeded in stirring up Pakeha suspicions and antipathies.

\section{The Prison Officer Group Within The Jails}

While these divisions were beginning to occur across the prison as a whole, the way in which its physical layout allowed for the emergence of two separate jails further reinforced these divisions. Table 4.4 provides an overview of the general demographic structure of Alpha and Beta Jails as at the start and end of the research period. The proportions of each demographic category remained relatively stable for each jail with the exception of ethnicity. Alpha Jail increased its proportion of Pakeha officers while Beta Jail increased its proportion of Maori officers. 
Each Day is Different: Prison Officers and Their Work

\section{Table 4.4}

Demographic Details for Alpha Jail and Beta Jail Prison Officer Groups' Population as at the START (mid 1997) and END (late 1998) of the Research Period

\begin{tabular}{|c|c|c|c|c|c|c|c|c|}
\hline \multirow{2}{*}{$\begin{array}{l}\text { Demographic } \\
\text { Category }\end{array}$} & \multicolumn{2}{|c|}{$\begin{array}{c}\text { Alpha Jail } \\
\text { [START] }(\mathrm{N}=58)\end{array}$} & \multicolumn{2}{|c|}{$\begin{array}{c}\text { Alpha Jail } \\
\text { [END] }(\mathrm{N}=56)\end{array}$} & \multicolumn{2}{|c|}{$\begin{array}{c}\text { Beta Jail } \\
\text { [START] }(N=100)\end{array}$} & \multicolumn{2}{|c|}{$\begin{array}{c}\text { Beta Jail } \\
\text { [END] }(\mathrm{N}=93)\end{array}$} \\
\hline & (47) & $81 \%$ & (47) & $84 \%$ & (83) & $83 \%$ & (77) & $83 \%$ \\
\hline Females & (11) & $19 \%$ & (9) & $16 \%$ & (17) & $17 \%$ & (16) & $17 \%$ \\
\hline Pakeha & (37) & $64 \%$ & (38) & $68 \%$ & (55) & $55 \%$ & (48) & $52 \%$ \\
\hline Maori & (16) & $28 \%$ & (12) & $21 \%$ & (36) & $36 \%$ & (37) & $40 \%$ \\
\hline Pacific Island & (5) & $9 \%$ & (6) & $11 \%$ & (9) & $9 \%$ & (8) & $9 \%$ \\
\hline $0-4$ years service & (27) & $47 \%$ & (27) & $48 \%$ & (35) & $35 \%$ & (32) & $34 \%$ \\
\hline 4-10 years service & (23) & $40 \%$ & (21) & $38 \%$ & (50) & $50 \%$ & (46) & $49 \%$ \\
\hline 10+ years service & (8) & $14 \%$ & (8) & $14 \%$ & (15) & $15 \%$ & (15) & $16 \%$ \\
\hline
\end{tabular}

Note. Percentages rounded, totals may not equal $100 \%$.

Table 4.5 provides an overview of the demographic distribution of Alpha and Beta Jails as identified by the questionnaire. The differences between the New Zealand prison officer groups and the findings of the international research literature were becoming even more distinct between the two jails. When separated, Alpha and Beta Jails only resembled the total prison demographic structure in terms of age group and education level distributions. Differences between the two jails were that Beta Jail had higher proportions of women, non-Pakeha and probationary officers. Alpha Jail had higher proportions of males, Pakeha, 10+ year service category and officers with military and additional jail experience. In other words, Beta Jail had a higher concentration of newer style officers while Alpha Jail had a higher concentration of the traditional officer set. Given the choice to transfer to a particular jail lay with the officer concerned, the subsequent differences in the demographic composition of the two would suggest some intentional grouping. That is, the higher or lower proportions of particular demographic categories within each jail were the result of underlying preferences within the coalescing officer groups. Further, as my research revealed, these differences were being solidified and perpetuated because, when the opportunity presented itself, officers were recruiting their own from within the general officer population: 
Yeah friendships are built up with fellow staff and that, and like we know that we're losing [resigning officer's name], and you know, having to maintain all those balances with different people, so we go and ask our mate if he wants to come up [to Alpha Jail]. It's just better when you can work with your mates and (.) you know.

- Male, 10+ years service, Alpha Jail.

As this extract suggests, like-minded others were intentionally sought from throughout the prison. Therefore the disparate grouping was not simply the choice of individual officers or chance; rather, the influence lay with the recruitment practices of the particular jail's residing officers. Due to the different demographic structures, different work cultures became manifest in the two jails:

You only have to listen to the radio to see the difference between [Alpha] and [Beta] jails. You could be down in a [Beta] unit all day and you might only hear one or two messages come across the radio in three hours. Whereas here (Alpha Jail) the radio's going off (a lot of communication).... I think it's the culture of the place rather than the staff. I think you can place [Beta] jail staff up here and they'll interact the same as we are. It's just the culture of the work and the regimes we have up here that forms that bond and group.

- Male, 4-10 years service, Alpha Jail.

Interestingly, as demonstrated in this extract, officers typically associated the emerging differences in cultures with the nature of the work and regimes rather than a reflection of the staff groups within the two jails. I would contend that both were influential. Notwithstanding this, the sociology of prisons literature has increasingly recognized multiple staff cultures and tends to also locate this within and across prison types rather than as a reflection of the staff that gravitate within these environs. In particular, the literature notes that there are more relaxed staff interactions within non-maximum security institutions (see Crawley, 2004; Williams \& Soutar, 1984). Alpha Jail was not formally a maximum security facility, but because it had the remand unit, the accommodation unit with the anecdotal reputation as the most violent in New Zealand and because of its more traditional early twentieth century architectural design, different relationship bonds and boundaries and work practices had began to emerge in it when compared to Beta Jail; similarly in the types of officers who were attracted to each jail - and subsequently, the types of officers the residing officers sought to include in their group. To illustrate that the emerging cultures of the two jails resulted from both the nature of the work and regimes and 
residing staff types, consider ethnicity. Given the differences between Maori, Pakeha and Pacific Island peoples, it would be expected, as there was, that there would be a difference in ethnic distribution between the two jail types. For example, while Alpha Jail was physically confined, promoting close working relationships, in reality the duty and routines meant that officers were less involved with intimate contact or interaction and were more mechanical in association. It would thus be expected that this environment would be more amenable to the Pakeha ethos of individualism. Beta Jail on the other hand, with its relaxed atmosphere and congenial surroundings, promoted a community style atmosphere with officers and inmates interacting more often at personal and meaningful levels and therefore more conducive to the Maori and Pacific Island peoples' manner of interaction. Further, as Beta Jail contained the two ethnic officer cliques, it should not be surprising to find a higher proportion of non-Pakeha and 4-10 years service category within it. 
Each Day is Different: Prison Officers and Their Work

\section{Table 4.5}

Jail Type: Demonstrating the Proportion of Each Demographic Category Within Each Jail Type

\begin{tabular}{|c|c|c|c|c|c|}
\hline \multirow{2}{*}{\multicolumn{2}{|c|}{ DEMOGRAPHIC CATEGORY }} & \multicolumn{4}{|c|}{ JAIL TYPE } \\
\hline & & \multicolumn{2}{|c|}{$\begin{array}{l}\text { ALPHA JAIL } \\
(\mathrm{N}=45)\end{array}$} & \multicolumn{2}{|c|}{$\begin{array}{c}\text { BETA JAIL } \\
(\mathbf{N}=70)\end{array}$} \\
\hline \multirow[t]{8}{*}{ UNIT LOCATION } & Yellow & (15) & $33.3 \%$ & $(0)$ & $0.0 \%$ \\
\hline & Orange & (16) & $35.6 \%$ & $(0)$ & $0.0 \%$ \\
\hline & Green & (14) & $31.1 \%$ & $(0)$ & $0.0 \%$ \\
\hline & Pink & $(0)$ & $0.0 \%$ & (12) & $17.1 \%$ \\
\hline & Lime & $(0)$ & $0.0 \%$ & (17) & $24.3 \%$ \\
\hline & Grey & (0) & $0.0 \%$ & (14) & $20.0 \%$ \\
\hline & Purple & $(0)$ & $0.0 \%$ & (14) & $20.0 \%$ \\
\hline & Excluded & $(0)$ & $0.0 \%$ & (13) & $18.6 \%$ \\
\hline \multirow[t]{2}{*}{ GENDER } & Male & $(40)$ & $88.9 \%$ & $(57)$ & $81.4 \%$ \\
\hline & Female & (5) & $11.1 \%$ & (13) & $18.6 \%$ \\
\hline \multirow[t]{3}{*}{ ETHNICITY } & Pakeha & (30) & $66.7 \%$ & $(42)$ & $60.0 \%$ \\
\hline & Maori & (11) & $24.4 \%$ & (21) & $30.0 \%$ \\
\hline & Pacific Island & (4) & $8.9 \%$ & $(7)$ & $10.0 \%$ \\
\hline \multirow[t]{3}{*}{ SERVICE LENGTH } & 0-4 years & (21) & $46.7 \%$ & (32) & $45.7 \%$ \\
\hline & 4-10 years & (14) & $31.1 \%$ & (28) & $40.0 \%$ \\
\hline & $10+$ years & (10) & $22.2 \%$ & (10) & $14.3 \%$ \\
\hline \multirow[t]{7}{*}{ AGE GROUP } & 20-24 years & $(0)$ & $0.0 \%$ & $(2)$ & $2.9 \%$ \\
\hline & $25-29$ years & (6) & $13.3 \%$ & (5) & $7.1 \%$ \\
\hline & 30-34 years & (11) & $24.4 \%$ & (15) & $21.4 \%$ \\
\hline & $35-39$ years & (9) & $20.0 \%$ & (18) & $25.7 \%$ \\
\hline & 40-44 years & (10) & $22.2 \%$ & (15) & $21.4 \%$ \\
\hline & $45-49$ years & (6) & $13.3 \%$ & (12) & $17.1 \%$ \\
\hline & 50-54 years & (3) & $6.7 \%$ & (3) & $4.3 \%$ \\
\hline \multirow[t]{5}{*}{ EDUCATION LEVEL } & None & (8) & $17.8 \%$ & (9) & $12.9 \%$ \\
\hline & Low Secondary & (10) & $22.2 \%$ & (19) & $27.1 \%$ \\
\hline & High Secondary & (7) & $15.6 \%$ & (8) & $11.4 \%$ \\
\hline & Low Tertiary & (18) & $40.0 \%$ & (31) & $44.3 \%$ \\
\hline & High Tertiary & $(2)$ & $4.4 \%$ & (3) & $4.3 \%$ \\
\hline \multirow[t]{6}{*}{ JoB TITLE } & Probationary Officer & (7) & $15.6 \%$ & (18) & $25.7 \%$ \\
\hline & Prison Officer & (20) & $44.4 \%$ & (19) & $27.1 \%$ \\
\hline & Instructor Officer & (4) & $8.9 \%$ & (12) & $17.1 \%$ \\
\hline & Unit IC & (12) & $26.7 \%$ & (16) & $22.9 \%$ \\
\hline & Unit Manager & $(2)$ & $4.4 \%$ & (4) & $5.7 \%$ \\
\hline & Missing Variables & & & (1) & $1.4 \%$ \\
\hline \multirow[t]{3}{*}{ MILITARY EXPERIENCE } & Military Experience & (16) & $35.6 \%$ & (21) & $30.0 \%$ \\
\hline & No Military Experience & (28) & $62.2 \%$ & (49) & $70.0 \%$ \\
\hline & Missing Variables & (1) & $2.2 \%$ & & \\
\hline \multirow[t]{3}{*}{ ADDITIONAL JAIL } & No Experience & (29) & $64.4 \%$ & (50) & $71.4 \%$ \\
\hline & Additional Experience & (15) & $33.3 \%$ & (19) & $27.1 \%$ \\
\hline & Missing Variables & (1) & $2.2 \%$ & (1) & $1.4 \%$ \\
\hline
\end{tabular}

Note. Percentages rounded, totals may not equal $100 \%$. 
The jails had the effect of dividing the prison into two large sub-groups. This was evident from group loyalties within each jail type and perceptions held by officers about the 'other' jail type. Alpha Jail and Beta Jail officer groups perceived their role, duties, experiences and demographic structures differently. For example, consider association time, that period in the evenings after dinner when inmates were able to associate freely together within their unit. In Alpha Jail, this typically involved inmates milling about in the dining room, playing cards or chess or associating in each other's cells. In Beta Jail, association time was generally the occasion for playing ball games in the compound, chatting in groups at the picnic tables or associating in each other's cells. Alpha Jail generally locked down at 7pm while Beta Jail generally locked down at 8.30pm. During evening association time, Alpha Jail officers tended to be involved in vigilant observation of inmates; routine tasks of moving segregation inmates around the unit without contact with mainstream inmates; locking down specific categories of inmates at varied intervals; clearing dishes from separates and early lock inmates; processing courts; and receiving, interviewing and housing new arrivals or inmates transferring from other units. On the other hand, in Beta Jail, officers would often engage in inmate activities such as sitting in the compound playing guitars and singing; playing pool or cards; partaking in weights workouts; playing ball games; having a cup of tea and a casual chat at the picnic tables or in cells and so on.

Officers were certainly aware of the differences between Alpha and Beta Jails as working environments and without exception each had a preference for a particular style. For example, to Alpha Jail officers, Beta Jail was characterised by boredom, frustration, laziness and predictability:

I like being busy. I find that I can go home and feel I've achieved something for the day. [Beta] jail I found quite frustrating cos there's not a great deal that you can do down there, you know. Now it's not being personal with officers (in Beta Jail), but they're lazy. Whereas up here (Alpha Jail) people work. I like working, it's good. I like to work, do things. You know you've worked eight hours rather than 'what the hell am I doing here?' God how boring would that be.

- Female, 0-4 years service, Alpha Jail. 
In contrast, Beta Jail officers' view of Alpha Jail was that it was pathologically claustrophobic compared to the much greater sense of space, personal freedom and relaxed atmosphere that the design of their jail gave them:

\begin{abstract}
Working up [Alpha Jail], to me it's a lot more claustrophobic and unhealthy. To me it seems old, dirty, particularly [Green Unit], it's not ventilated, it's not a good environment to work in. In the winter it's (Alpha Jail) just a germ centre! Here (Beta Jail) you've got that space, you've got space to get out of the road. Space gives people perspective, you can view your position, you're not in each other's face. You can go for time-out, you can go for a walk round the back or something... you notice how people with a confrontational 'in your face' attitude will soon mellow out coming down into the more open units - officers too (laugh).
\end{abstract}

- Male, 4-10 years service, Beta Jail.

As is common when there are distinct groups, there was an element of 'goading' between the Alpha and Beta officers, demonstrating the in-group-out-group split. While generally convivial, it did highlight antagonisms within the prison officer group. For example, Alpha Jail officers referred to Alpha Jail as the real jail and Beta Jail as the holiday camp:

Honestly I've only worked down there (Beta Jail) a few months, I couldn't stand it, the space, it made me uneasy... it drove me batty. I had to come back (to Alpha Jail) because this is the real jail. Down there it's a holiday camp yet they call themselves prison officers, you know, it's like, it's blasphemy. \{my emphasis\}

- Female, 4-10 years service, Alpha Jail.

Rather than acknowledge the emerging division in the staff group, officers could assert that Alpha Jail was the 'real jail' based on both its traditional physical design and the security driven routines which officers had to perform ${ }^{128}$. Given the higher security status of inmates relative to Beta Jail, Alpha Jail officers asserted that they needed to be hyper vigilant to potential disturbances. In this way, Alpha Jail officers proposed that their group rightfully contained greater proportions of more experienced and ex-military type officers. That is, those willing and able to take on the 'real criminals'. In contrast, Beta Jail supposedly reflected a weaker or disinterested staff group:

${ }^{128}$ For examples of Alpha Jail and Beta Jail shift routines see Appendix M. 
These are the units (Alpha Jail) where the suicidal people come in, the druggies first come to unwind, and all this stuff, we've got the real prisoners. If they (Beta Jail officers) have any problems (difficult inmates) they just send them up to us to deal with. \{my emphasis\}

- Male, 4-10 years service, Alpha Jail.

There were some mixed perceptions of who should be where, however. For example, given the Alpha Jail officers' generalised perception of their jail being the 'real jail', many of them felt it preferable to have more experienced staff in it to ensure security and control and preferred probationary officers to train in Beta Jail. However, as indicative of the varying and contrasting opinions emerging in post-restructuring prison life, an equal proportion of Alpha Jail officers felt that probationary officers should start in their jail as it would indicate whether they were capable of being an officer or not:

\begin{abstract}
My philosophy is that to be a good officer you have to be able to work in [Alpha Jail] first... (there are) officers who go down to the [Beta Jail] and get on quite well with the inmates, and then come up here and face a totally different environment. It can actually be quite scary (for them to adjust).
\end{abstract}

- Female, 4-10 years service, Alpha Jail.

This divergence of opinion, even within jail type, was indicative of the overall lack of cohesion within the officer group. As it was, the greater proportion of probationary officers, 72\%, were actually in Beta Jail. Similarly, Beta Jail had a higher overall proportion of probationary staff with $25.7 \%$ of their complement compared to $15.6 \%$ of Alpha Jail.

It was asserted that senior staff would prefer to be in the 'real jail' as the tasks there constituted more traditional—'real'—prison officer work. Indeed, with regard to job title, a greater proportion of Alpha Jail identified as Unit ICs. Similarly, Alpha Jail had a higher proportion of $10+$ years service length officers relative to Beta Jail. Thus, perceptions matched demographic spread in the assertion that more seemingly 'hard core' officers were in the 'real jail'. Accordingly, when Alpha Jail officers disparagingly referred to Beta Jail as the 'holiday camp' it was not simply due to its physical design. It was asserted that Beta Jail was where officers were inclined to go 
for respite from the 'real jail' and subsequently spend the majority of their time gratifying inmates’ needs rather than being focused on control and security:

For some people it's a good thing to go down there (Beta Jail). For me, I needed a break so I went down there but I (had to) come back up here, it didn't help me. - Male, 4-10 years service, Alpha Jail.

In contrast, Beta Jail officers thought that Alpha Jail officers did not understand the difficulty of the open design in relation to security and control and the additional pressures this placed on them. While routines may not have been as focused on security as in Alpha Jail, additional responsibilities such as work gangs, programme attendance, case management, programme assessments, parole board reports and so on, as well as different types of inmates, did place a variety of different pressures and strains on these officers:

\begin{abstract}
Every unit's got its own stress levels but they're all different. Up [Alpha Jail] the inmates are all in your face, but here (Beta Jail) there's so many white collar crime people that paper people (file official complaints). So if they don't get their own way with one officer they put it down on paper. I say I'd rather have those guys up there (Alpha Jail inmates) who are bloody robbers and that and you can have a blimmin good argument and it's good and over. Here it starts with the old pen.
\end{abstract}

- Female, 4-10 years service, Beta Jail.

Even Alpha Jail officers acknowledged that they preferred the more 'traditional' approach of Alpha Jail inmates than the modern approach found in Beta Units. It was as if the different style of the officers (newer style versus traditional style) was mirrored by the inmates' style within the respective jails. Further, the case management tasks within Beta Jail gave these officers a vested interest in the future prospects of their case inmates, an attachment or emotional investment which Alpha Jail officers were unlikely to have:

I'd like to think I have made a difference. I've seen a few guys (ex-inmates) that I've had a lot to do with in here (Beta Jail), and you know, they've been in jail a couple of times and I've spent a lot of time with a few of them. And I've seen them on the street lately, and they're working, you know, they've got partners. They'd split up when they were in jail, but they're happy, you know, things are going really well for them. You feel good about that. But it's always a real kick in the arse when they come back. I always take it really personally, a stupid thing, but I do. You invest a bit of time, I tell them 'you've let us down, you told 
me you'd do this but you haven't', and there's always the same old bullshit excuses and I say 'it doesn't cut it, come here', boot (imaginary kick) that's the thing I always tell them, 'come back and I'll boot your arse, you stick to your word'.

- Male, 4-10 years service, Beta Jail.

As this extract demonstrates, at one level an emotional investment in the progress of an inmate could be positive, but then there was always the potential of disappointment when they saw the inmate coming back in. Goffman (1959) asserted that when inmates became objects of feeling and affection social distance diminishes. If an inmate then does not perform to the officer's expectations, the staff member would interpret this as a serious assault on their self as their perception of the inmate is that he would be 'reasonable'.

Finally, Beta Jail officers also thought their group needed to contain the entire spectrum of officers for the varied duties they needed to perform. In these respects, there were a greater proportion of women in Beta Jail with $18.6 \%$ compared to $11.1 \%$ in Alpha Jail ${ }^{129}$. Traditional gender role division would suggest that women would prefer the tasks associated with duty in Beta Jail as it included case management and less confrontational personal interactions. However, women officers' motivations for employment were more pragmatic than altruistic ${ }^{130}$. It was more likely that the less frenetic nature of the job in Beta Jail made it more attractive to them.

Overall, the social arrangements which officers referred to as 'the good old days' and the research literature as the 'typical prison culture' had lost its mode of transmission. Instead, a new set of social arrangements based around these features was coming into existence, framed around divisions and difference rather than unity and cohesion. These developments were occurring on top of the way in which the division of the prison into jails led to each one performing relatively different functions with relatively different security classifications. Each jail began to take on its own characteristics and identities - attracting different types of officers. These divisions

\footnotetext{
129 This was an expected outcome given that nationally the more traditional design prisons showed greater proportions of male officers, such as Dunedin, Invercargill, Waikeria, Manawatu and Wanganui Prisons. See Appendix L for the national distribution of women officers.

${ }^{130}$ As indicated by analysis of the questionnaire (motivations for becoming a prison officer) shown in Appendix O, Table O.2.
} 
were beginning to take effect right across prison officers' working routines and were reshaping the culture that had hitherto sustained them in their work. Previous arrangements could no longer be taken for granted. 


\section{CHAPTER FIVE: LEARNING THE SCRIPTS}

Some of the immediate consequences of this erosion of the homogeneity and unity of the officer body were reflected in the training of new recruits to the service. Traditionally, this training had consisted of a two-step process. In the first, the civilian applied for a position as a prison officer, experienced a brief period of 'scoping' in the prison, and then joined the service as a probationary officer. Second, the civilian, now in uniform, had to learn how to 'do' the job, and more importantly - how to 'be' an officer. Therefore, during the twelve month probationary period the new recruit not only had to learn the formal requirements of the job, but also had to become familiar with the traditional officers' script. Thus, all probationary officers had to learn the informal prison argot and nuances of officer language, learn the occupational culture and expected norms of behaviour, pass a series of character tests and successfully manage character defining incidents. The expectations placed on individuals during this process by officers varied according to the gender and ethnicity of the probationary officer. However, at the end, if the probationary officer survived, they were formally awarded the stripe on their epaulette and thereby 'got the bar up ${ }^{131}$ and could be considered an officer. Their concept of self would have been rewritten so that they could accommodate and perform the role 'the script' had assigned to them.

However, as a result of the increased competition and antagonism within the officer group in the aftermath of the early 1990s policy changes, two significant problems were beginning to jeopardise the dynamics and success of what had become a wellestablished process. In the first instance, the changing demographic structure of the prison officer group meant that inexperienced officers were increasingly responsible for the mentoring of probationary officers, as there simply were not enough senior officers to do this task (there were around 30 new recruits during the course of my research and this number was increasing as additional units were opened in Beta Jail;

\footnotetext{
${ }^{131}$ Getting 'the bar up' was the colloquial term for being awarded the stripe on one's epaulette that denoted movement from probationary officer to prison officer at twelve months. This was a significant event given there really was no other promotional moment.
} 
there were approximately the same number of officers leaving, recall Table 4.2). This resulted in a varied transmission of the traditional officer script, given that the inexperienced officers were not familiar enough themselves to teach it. In addition, some senior officers were reluctant to assist in the training of probationary officers whom they deemed unworthy of their efforts, given that in their view they did not possess the appropriate attributes for the job and hence were unlikely to survive. This was particularly the case if the probationary officer had been attracted to the job by the prospect of excitement and challenge, which had been part of the mid 1990s advertising campaign.

Secondly, there was no longer just one script for probationary officers to learn. In the past, a traditional script which had been passed down through generations of officers had regulated officers' work. It was simple, functional and known and it provided a means of interpreting the formal prison rules and complemented the inmates' cultural script, thereby enabling predictability within the prison (see Sykes, 1958). However, the changes in prison administration and prison officer working practices was leading to the emergence of a new script, which also bore a heavy imprint from Corrections Head Office.

Despite its rejection by the existing officer group and all that it involved (such as roles for ethnic groups and women), recruits were being attracted to the job by the promises of challenge and excitement that the new script offered and began adopting aspects of it. Paradoxically though, these probationary officers were still largely trained within the traditional script. However, as increasing numbers of recruits with differing backgrounds, motivations and ethnicities joined the service, groups of officers began to conduct themselves according to variations of the old and new scripts. This created two problems. The first was that it effectively removed the stability created by the traditional script while not establishing a singular new script to replace it which all the officers would accept. Secondly, with multiple scripts to learn, prison officer training became much more difficult:

You know they all blame me for [details of an incident] but it wasn't my fault. I didn't know he was on locks (off privileges) and it's not like I've even been to college or (laugh) probably never get there now.... I blame [inmate's name], bastard, he knew I'd let him through (to restricted area). If they (officers) 
wouldn't keep changing the messmen like everyone knows? How was I to know? It's the end of me though eh. [Senior officer's name] and [senior officer's name] have it in for me... they forget how hard it is and it's not like they help me any. How embarrassing eh, won't get the bar up at this rate. Least I made it this long. I've heard some women don't last a week \{my emphasis\}

- Female, 0-4 years service, Beta Jail.

This extract demonstrates the precarious position probationary officers found themselves in. Inmates were aware of the developing factions brought about by restructuring and took advantage of the confused state of the probationary officers. Longer serving officers were reluctant to assist, but at the same time were quick to highlight any of their perceived inadequacies or errors. Meanwhile, when a probationary or new officer left the service, this merely reinforced the senior officers' impression that these 'new style' officers were not suitable for the job or worthy of their instruction - cementing a self-fulfilling prophecy.

This chapter will demonstrate that as a result of the policy and demographic changes of the early 1990s, learning how to 'do' the job was becoming increasingly complicated and the well-established process of acculturation was beginning to break down. While the formal rules were taught at the Prison College the informal rules, taught on the shopfloor, had a greater bearing on day-to-day prison life and needed to be learnt quickly if the probationary officer were to survive. Due to a lack of, and unwillingness by, longer serving officers, inexperienced officers were charged with teaching the informal rules to the probationary officers leading to the inconsistent transmission of the traditional officers' script. This was further complicated by the longer service officers' rejection of the new script imposed by Head Office and the varied acceptance of parts of both scripts by the new style Pakeha, ethnic and female officers. The multiplicity of scripts that had emerged complicated the day-to-day working of the prison as all officers had to be at least familiar with them if they were to 'get the job done' and 'get along' with each other. At the same time, the probationary officer was expected to get the basics, according to the traditional officers' script, right, develop a suitable style or way of being in the prison and display appropriate loyalty to their fellow officers. Regardless, if the probationary officer survived their first twelve months, a new 'prison self' emerged, they got their 'bar up', and they were then a prison officer, but one who had been through the 
training experience with enduring effects on them. For probationary officers, the central difficulty was developing their prison self while witnessing and learning a multiplicity of scripts which had weakened the previously culturally prescribed ways of being. For longer serving officers, the multiplicity of scripts complicated their previously 'known' existence and alienated them from these new style officers as they could no longer trust them. In many respects the traditional officers' script was still the overarching guide for informal life; however, as a result of its weakened state, it was the inconsistent adherence to it now that was to make prison life more painful for all officers.

\section{Scoping}

The formal or 'ideal' qualities which a new recruit had to have included: a class B (car) driver's licence; no prior criminal convictions; New Zealand citizenship (or permanent residency); and a current first aid certificate. Within the application process the potential recruit had to pass: a prison officer selection test ${ }^{132}$ (POST); a general intelligence test ${ }^{133}$; a psychological test ${ }^{134}$; a medical examination; and a basic fitness assessment. Other than seeking non-sexist and bicultural attitudes and behaviour, there were no other formal expectations placed on the new recruit ${ }^{135}$. Their first days inside a prison were part of a scoping exercise. This was where potential recruits spent one to five days within a local prison, attached to an officer, observing the job in action. Given the brevity of this exposure and the foreignness of the environment, potential recruits characteristically formed a false impression of prison work. Similar to my first few weeks on observation for this research, the scopee

\footnotetext{
${ }^{132}$ According to officers, this test involved putting punctuation in basic sentences and solving simple arithmetic equations. This would seem to be confirmed by the Ministerial Committee (1989, p. 77) which listed as criteria "satisfactory performance of an arithmetic test; and the ability to write a short essay on a selected topic which assesses spelling, grammar, sentence construction, word fluency and the building of ideas and concepts”. The essay component was removed in 1990 as recommended by the report (idem).

${ }^{133}$ The intelligence test was the Ravens Progressive Matrices test (Ministerial Committee, 1989, p. 77).

${ }^{134}$ The psychological test was not named in the recruitment documentation or Ministerial Committee (1989) report. It would appear that this test was to measure non-sexist and bicultural attitudes and behaviour (ibid, p. 78).

${ }^{135}$ The basic physical requirements of: preferably between 23-40 years of age; over 5'8', in height; and no need for the use of spectacles except for reading - were removed following the Ministerial Committee (1989) report.
} 
would witness officers seemingly constantly joking about with each other and with inmates. There appeared to be a lot of activity and movement. This would confirm to the scopee that the job was interesting, challenging and exciting-that every day would indeed be different and challenging-quite different from the reality of the mundane routines which gradually revealed themselves and also the divisions within the officer body.

Who was provided as the mentor for the scopee was of great significance. This frequently had a bearing on which, if any, group or clique the individual would subsequently be accepted within and which allegiances the new officer would develop. The reason for this was that, typically, one's scope officer became one's induction officer when one was formally placed on probation. Even after their brief period of scope, however, civilians still had no real conception of what it was to be an officer or to do prison work. As Goffman (1968) argued, the newly stigmatised is typically unaware of the effects of their stigma until closely affiliated with likesituated others - hence the scopee would have no conception of the realities until they were on probation for some time. Regardless, if the civilian then accepted a position within the service, s/he had progressed through step one and was no longer a new recruit but was now a probationary officer.

It seemed that few of the serving New Zealand officers at the target institution had had realistic pre-recruitment images of prison work (see also Lombardo, 1981). Even those who had relatives and friends working within the system had erroneous views of what it constituted. For example:

I've got to say when I first looked at starting here I didn't know much about it. I had no concept apart from what you see on TV, you know, the old sort of jail in that stupid Australian thing ${ }^{136}$. You get the completely wrong idea of it. Apart from getting inmates out of cells I really didn't have any idea beyond that. But I liked working with people... you don't expect to have so much inmate contact as you actually do, which I don't actually mind, but you know, you see all these American things where they're in their cells most of the time - it's not like that... you're not just opening doors, locking doors. You're a guidance counsellor, social worker, you know, a shoulder to cry on for some of the guys. So you're not just a turnkey, there's just so many facets to the job. I had no idea.

- Female, 0-4 years service, Alpha Jail.

\footnotetext{
${ }^{136}$ Australian serial television programme called ‘Prisoner', televised in New Zealand.
} 
Officers were well aware that the Department of Corrections were seeking demographically different officers from those in the longer service group. Longer serving officers referred to recruitment drives in typologies that matched popular sports. For example in the 1980s the 'type' was golf players ${ }^{137}$, that is, white, lower middle class, physically strong but not overpowering males. In the early 1990s the ‘type’ was rugby players, hence 'brown', working class and physically commanding males. Officers recognised that there was now a difference in the new recruits they were encountering but found it difficult to describe, apart from Head Office seeking 'business types' or a movement 'from brawn to brains', qualities which they judged to be unsuited for the job:

\begin{abstract}
Oh we've gone through a few different sports here. I'll give you an example, when I was first introduced to the chief officer, when I first joined, the first question he asked me was 'do you play golf?' And I says 'yes, I’ve just joined the golf club'. And that was it, straight up, no shit, 'do you play golf?' was the first question he asked me. Not 'why did you join the service?' or 'what are you looking forward to?' And that was my boss at the time! In my day it was golf because it was the higher-class white European male played golf. There was a period of time when the prison employed a great number of rugby players. It (the prison authorities) wanted big tough guys all right. They did like rugby players, undoubtedly, it was a key in the door. I remember we had a huge intake one year, eight or ten, all rugby players.... Now the new guys (pause) must be tiddlywinks (laugh).
\end{abstract}

- Male, 10+ years service, Beta Jail.

On the whole, officers felt less secure in the wing with the new style of officer, as they questioned their ability to react to conflict:

\begin{abstract}
They're hired now for different skills and attributes they have, whereas the older guys were hired because they were a rugby player and Polynesian (Maori or Pacific Island). And if you're a rugby player you're a team player. It was a very physical job when I started. Everything was very physical and was done in a way that was, well you had to show a bit of form every now and then. But new officers, I don't know that some of them can handle it very well. I think there's still a place for that kind of officer (new type) but at the end of the day that officers caught up on a lot (physical inability is revealed). When it comes to a sticky situation an officer from the computer isn't going to help you at all. And it happened to me, I've had an officer run on me (not backup during an incident).
\end{abstract}

\footnotetext{
${ }^{137}$ In New Zealand, in contrast to other countries, golf club membership has never been exclusive to the upper classes. Annual membership fees range from $\$ 500$ (full eighteen hole course with catered grounds) to \$2000 (internationally recognised links courses). Casual game fees for non-members ranged from a donation in an honesty box to $\$ 50$. New Zealanders from any background can be found at any course with the only clothing requirement being soft sports shoes and a collared t-shirt devoid of liquor advertisements.
} 
And I said 'you need to be thinking about whether you want to be a prison officer or not'... he left the job.

- Male, 4-10 years service, Alpha Jail.

Here, again, this officer reveals how a new, likely unsupported, officer was ill prepared for the job, had his 'inadequacies' exposed, and promptly left - reinforcing the longer serving officers' belief that this 'type' of person was not physically suitable. Indeed, the types of new recruits longer serving officers wanted to see more of were ex-military, while mid-length serving officers wanted to see more nonPakeha. In other words, more physically commanding and disciplined officers:

Being ex-military is a great asset because you're very fit, and you're also disciplined, and you're big (commanding presence), so that's great, you need that sort of person here.

- Male, 10+ years service, Beta Jail.

The inmates won't listen to European officers. And that straightaway makes it hard to do your (all officers) job, you know. Therefore why are they hiring more European officers now?

- Male, 4-10 years service, Alpha Jail.

In contrast, some of the officers (particularly newer officers) thought that the new style of officers would produce positive results for the prison service claiming that there were already fewer violent incidents:

I think there is an improvement with the new style officers that are coming in. The days of bashing inmates have gone, you know. It's sort of a different approach. Being able to communicate and talk makes it easier. It's a lot easier talking to these guys (inmates) than sort of pushing it to a point (antagonise the inmates as alleged in the past).... There seems to be getting less and less take out's (C\&R) over the years, so I guess they (new style officers) must be doing something right in how they're being prison officers. So yeah, it's that change, the violence in here is getting a lot less.

- Female, 0-4 years service, Alpha Jail.

As can be seen from the above three extracts, the image of 'who' would make a good officer and the effect of the new style of officer varied significantly between the three lengths of service groups residing in the prison, each, with a logical self-enhancing bias, tending to prefer the 'type' that was popular when they were recruited. 
Regardless of these differing perceptions, the new style officer was a reality in the prison and officers of all lengths of service had to adjust to this. However, because of the reduced commonality that the officer group now enjoyed, this acculturation process was becoming problematic. The traditional script still existed, but the new officers were reluctant to operate under its influence - this was not what they had joined the prison service for. Instead, newer style officers selected the parts of both the old and new scripts which they were comfortable with, creating a multiplicity of available scripts. Longer serving officers were reluctant to accept the newer officers' interpretations of the traditional script, adding to the division between the two groups.

I asked experienced officers (over two years on the job) what they thought were the most valuable qualities that prospective officers should possess. That is, what were the 'real' rather than ideal qualities which would make the new recruit a 'good officer'. None of the qualities they mentioned were the same as those which Head Office wanted. Their responses illustrated an awareness of the complexity and precariousness of the position of prison officers. They also demonstrated a difference in required qualities depending on the ethnicity or gender of the new recruit. For example, if the new recruit was visibly non-Pakeha, the only required quality expected by the existing officer group was for the person to have a commanding physical presence in the wing. Acceptance by one of the ethnic groups which had formed would then be automatic:

\footnotetext{
I was quite lucky. I started up here when we used to call it 'The Bronx' (Green Unit). We had a muster of 72. You're supposed to have two officers in the wing. Well most of the time we only had one. But I could walk down a wing and be quite comfortable doing that whereas other staff may not have been as comfortable as I was. I suppose me being Maori helped a lot.
}

- Maori, 4-10 years service, Alpha Jail.

Having a physically commanding presence, albeit 'brown' skin, fitted with the traditional officers' script of what it was to be an officer. Alternatively, women were expected to know their place, keep out of the way if a situation arose, and be the 'social worker' or 'mother' for inmates with problems. If, however, the new recruit was male and Pakeha, a variety of expectations existed. The requisite qualities for such recruits according to the officer group contained two general themes: the 
personal qualities of the individual and the qualities required for task fulfilment. For example, the most commonly cited required personal quality was that the person had had life experience which included exposure to different cultures, some level of hardship (a graduate from 'the school of hard knocks' idea) and an ability to communicate with working class people:

\begin{abstract}
I think you need to have some life experience. I honestly believe that people who are streetwise make very good prison officers because they know what's going on. It's the old adage, you know, don't kid a kidder! (They need to be) someone who's been on the edge, they've had to live out there and struggle, they know what it's like and what's going on. I'm afraid they (new recruits) haven't got a background where they've been around different cultures. They haven't got a grasp of what real people are.
\end{abstract}

- Male, 4-10 years service, Alpha Jail.

Thus, the new style male Pakeha officer, although more physically resembling the traditional officer group, was not perceived to have the requisite 'life experience'.

As regards the qualities required for task fulfilment, officers highlighted the need to have the right attitude for prison work. For longer serving officers this was reflected by such behaviours as the willingness to participate in prison life beyond the minimum required duties, and an appropriate balance between conscientious care for prisoners while maintaining objectivity:

\begin{abstract}
A lot of them (newer officers) haven't got the right attitude for the job. They think that they can just come in here and lockup and go home. Well it ain't that easy in here... (whereas officers) like myself (who have the right attitude), have lost a lot of sleep over worrying about 'at risk' inmates that have come in. Whereas they (new officers) don't think that's a problem (overly distance themselves from the human service aspect of the job), or otherwise they get too deep into a situation and they can't dig themselves out of it properly.
\end{abstract}

- Female, 4-10 years service, Alpha Jail.

Finally, new recruits had to demonstrate that they actually wanted to be a prison officer and all that this entailed. This was reflected in both demonstrating the right attitude and in their motivations for joining the service:

You need to have no hidden agendas... (you need to be) somebody who wants to be a prison officer and is happy to remain a prison officer for several years. Not (be somebody) waiting for the next thing to come along... if you know they've 
committed themselves, they'll commit themselves not only to their job, but to their colleagues. And (they'll) commit themselves to developing a rapport and a relationship with their colleagues. Whereas if they're here waiting for a vacancy (different job) they'll think 'what the fuck, I'll just bide my time here'. I can't stand that, I think it's so awful to see and very cheap. They're cheating their colleagues and the offenders... it's a loyalty thing! I want somebody who's going to give me that loyalty. I'm not talking about watching your back all the time and standing by you in thick and thin. I'm not talking about that. Just loyalty to the job.

- Male, 4-10 years service, Alpha Jail.

At the same time officers wanted recruits who would function as team members:

You've got to be like a rugby player instead of a squash player. What I mean by that is a team player, you know, like work in with all your mates.

- Male, 0-4 years service, Alpha Jail.

As these two extracts indicate, loyalty to the job, organisation and fellow officers meant that one was likely to be regarded as a worthy colleague. At the same time, this could be difficult for new officers to demonstrate when a significant number of them were largely excluded from many of the interactions due to the longer serving officers' preconceived perceptions of them as unsuitable.

Other desired qualities included the ability to be patient but quick thinkers, show initiative but not act without instruction (until one’s bar was up one was deemed incapable of making a correct decision without instruction) and be flexible while being consistent. The ways in which these characteristics could seem contradictory and the dilemma that this posed for probationary officers was recognised by longer serving officers:

You can be flexible as well as consistent... I suppose it's hard in a way to try and all work the same way with everybody, you know, being flexible the same way. I don't think that that would ever happen? I guess you can go so far, without breaking that rule (work in the grey areas of a regulation), then you're consistent and flexible. Yeah, then it should be fine, but you start going too far over and that's where trouble starts, that's where the inconsistency thing comes in. It's up to us (experienced officers) to teach them (new recruits). Yeah, I can see that it would be hard, but we all had to learn.

- Male, 4-10 years service, Beta Jail. 
While this extract demonstrates an appreciation for the ambiguous position probationary officers were in while learning what it was to 'be' an officer and 'do' the job, it also demonstrates the 'hard line' approach taken by many experienced officers whereby the probationary officer was simply expected to learn, irrespective of how this was to occur. Thus, while it was recognised by experienced officers that 'learning' how to do the job and be an officer was difficult, they failed to appreciate just how difficult this had become in the aftermath of the restructuring. Paradoxically, the probationary officers would have benefited from greater assistance than had been required in the past due to the increased complexity that now existed, rather than the withdrawal of assistance that they were actually experiencing.

Nevertheless, after their scoping, potential recruits would be offered a probationary officer position at one of the prisons within the region they had applied to. Upon accepting the contract the new recruit was given a uniform and a start date. They were now probationary officers. However, it was quickly made clear to the probationary officer that they were not yet an officer in either the eyes of their fellow officers or the inmates:

\footnotetext{
They (inmates) won't recognise an officer if he hasn't got any bars up because you're still a probation officer. Quite often you hear (inmates say) 'he hasn't a bar up, what would he know'. The inmates still relate length of service with the bars on your shoulder.
}

- Male, 4-10 years service, Beta Jail.

With length of service there's an instant trust (from officers). You can talk once you've got that bar up, you're confident.

- Female, 0-4 years service, Alpha Jail.

The importance of 'getting the bar up', as indicated in these two extracts, highlights another aspect that had become problematic in the aftermath of the Workman reforms. Formally the epaulettes were supposed to have been abolished. However, informally they had persisted given the significance of them as a visual indicator of the officer's status within the prison (see Goffman, 1959). Nevertheless, the probationary officer had to learn the routines and role of the prison officer before achieving their bar at twelve months. Progression from probationary officer to prison officer was based on surviving twelve months of service and passing examinations to gain the Probationary 
Prison Officers Certificate (PPOC). They did this by attending the six-week ${ }^{138}$ Department of Corrections College residential training course.

\section{Prison College And PPOC: Learning The Formal Rules}

Attendance at the Prison College occurred at a time suitable for the prison and college schedule, usually around six to nine months into the probationary year. The formal curriculum included: Acts and Regulations; Correctional Studies (brief historical overview of prisons in New Zealand); Human Development and Behaviour; and Communication Studies. While the purpose of college training was to learn the formal rules and regulations of New Zealand prisons, many officers reported that for them, its most important aspect was the relationships formed during the residential stay:

\begin{abstract}
When you start with someone, you go through those six weeks (at college) and you learn a lot about a person when you're there. You can sort of decide then 'oh shit yeah, I get on quite well with this guy or this girl and we have a bit of fun together'. It might have been a study mate or whatever, and you find you've got a mutual bonding. You can take it outside the job too. You can socialise together, you might take your own partners, introduce them, and you create a little group of your own. Like a little outside of the job family type of thing. I think it's quite important to, you know, to have close friendships within the job.
\end{abstract}

- Male, 4-10 years service, Beta Jail.

The bonds made at college were as significant as the bond the new recruit had with their scope/ induction officer. Officers could name all the other officers that they had attended college with and where in the prison system, or out of the system, they were now located. It was both beginning to be apparent to the probationary officer how important collegial relations were as they came to terms with their stigmatised identity, and also marked the reduction of outside relationships for the officer and their family as they began to establish their own 'prison family'. Indeed, when questioned about their college experience, without exception fellow attendees, C\&R

\footnotetext{
${ }^{138}$ The full course is six weeks. However, with the high number of trainees it has been common for the college to provide just the four week academic component while the physical component (C\&R training) was organised to occur at the trainees’ prison when numbers were viable for holding a course.
} 
training and the catering were the only positive memories; certainly none mentioned the book work (history, theory, Acts or Regulations) as interesting or beneficial.

Indeed, the major difficulty for probationary officers, particularly those that did not 'fit' the experienced officers' perception of what it was to be an officer, was that they were twice disadvantaged - they were neither being trained sufficiently on the job by experienced officers nor adequately equipped at college. What was certainly the case was that college training did not prepare the probationary officer in terms of 'street savvy', empathy, objectivity or commitment - and most certainly not in the traditional officers' script. The majority of officers told the probationary officers to participate in the PPOC as instructed, then return to their unit and do things the way they had been taught prior to college by officers. In short, it was best to forget everything they had learnt there:

\begin{abstract}
I knew so many guys (officers) before I'd finished college or started on the floor. Their advice from ten years in the job was 'go to college, do your stuff, answer the questions they want to hear, when you leave college you forget it and you run your unit as everyone else does because that's the way things work'. And that was the best advice they gave me. And that's the way the jail works.
\end{abstract}

- Male, 4-10 years service, Beta Jail.

However, it was at the college that the formal prison rules were taught and the probationary officer was instructed how to refer to the various manuals which guided behaviour within the prison. Nonetheless, probationary officers were not taught how to interpret the grey areas within each rule. This teaching occurred on the shopfloor and was as good as the skill level of those who mentored the probationary officer.

\section{Learning The Real Rules!}

While the formal rules ultimately bound the prison officer to behave within their confines or risk losing their job, the informal rules were those which guided the officer through each day and thus were of more immediate importance to the probationary officer. It was the informal rules which needed to be learned quickly if one was to survive: 
I've got used to it, you've got to get used to it quick. It's so different from what I thought it was going to be. It's different and every day is different. It's like living in your own little community. It's totally different. It's like a little village, with little rules and regulations and hierarchy and different ways of doing things. That's with the staff and the inmates as well.

- Male, 4-10 years service, Beta Jail.

Probationary officers had to 'get used to the informal rules quick' as it not only influenced the officer group but also the inmates. However, due to the lack of senior officers, the probationary officer's induction officer was frequently not a long serving officer and often their experience was limited to their current unit. As a result, the inductee was trained according to the script of the given unit, indeed of the given officer, and not the ways of the whole prison. As the induction officer provided comfort and relief in this new environment, the close bond and esteem which the probationary officer came to develop for their 'saviour' should not be surprising. They held a position of profound significance to the scope/ induction officer. Officers, even years later, accorded this individual considerable status and admiration. For example, it was common during my first few months at the target prison for the prison officers to introduce fellow officers to me as 'this is [officer's name], he was my induction officer. You listen to everything he says'.

With such guidance, probationary officers very quickly learnt that the formal rules had little bearing on the day-to-day activity of the prison. Rather, it was the informal rules or the cultural interpretations of the formal guidelines which were important. The regulations established the boundaries of behaviour while the informal rules explicated the practice:

\begin{abstract}
The formal rules are like the guidelines, it draws the line how far we go. The informal rules enforce the rules with a little bit of bending them. One is a guideline and one enforces it. For example, not narking on other officers doesn't mean you're not going to follow the procedure - it just means you're going to deal with the situation right there, right now, without other people.

- Male, 0-4 years service, Beta Jail.
\end{abstract}

Learning the informal rules came from watching experienced officers acting out their particular script and how they handled situations within the 'grey areas' of the manual. It was the probationary officers' responsibility to pick up the rules: 
Interviewer: It seems there's just so many little rules and all that, some of them are so confusing, how do officers learn them?

Officer: Just shut up and watch! That's the problem with a lot of the new ones (probationary officers), they're too busy talking and telling us what we're suppose to be doing and they're not learning.

- Male, 4-10 years service, Beta Jail.

Even though it would seem that learning the informal rules or 'grey areas' was easy‘just shut up and watch' - it was in fact fraught and exceedingly difficult for the probationary officer given the multiplicity of scripts they were witnessing. There was a general assumption that 'everyone knew' the rules, yet deconstructing them, even for experienced officers, could be hard to verbalise, let alone 'pick up' by a newer officer. What further complicated the learning of the informal rules was the lack of consistency between units with their different interpretations and applications of them:

This job runs in the grey areas, and the grey areas are unique to each institution, unique to every unit. Within this jail every unit is unique in its own way and its way of running things and so the only way you gain that is by experience. Knowing the black and white but being able to blend the grey and knowing the most important thing is to cover your back.

$$
\text { - Male, 4-10 years service, Alpha Jail. }
$$

Indeed, there could be variations in the script being used depending on whom was on shift:

We change rules day by day. Really! In our job, depends who you're on with. Yeah, that's got a lot to do with it. The types of attitudes (of different officers) that you're on with changes how you're going to run those rules. So you just change them to suit the people that you're on with really. I reckon it's whatever goes the easiest is the easiest way to work.... I think if management found out how we run our own units in our own individual ways they'd be horrified, but at the end of the day the results are there, you know.

- Female, 4-10 years service, Alpha Jail.

Having said this, it seemed that there still were three essential informal rules which overrode any particularised differences between these different versions of the prison officer script. These were: 


\section{(i) Getting The Basics Right}

There were two essential aspects to getting the basics right. The first was to prioritise security above any other perceived goal of prison work. The second was to develop an awareness of the importance of communication between officers and the significance of all information, even the most minute and seemingly trivial detail. Thus:

I see the basics as the communication skills with each other, passing on information, doing musters, questioning everything, that's the basics, you know. Making sure you're securely containing them (inmates). Making sure that's what they're (inmates) meant to be doing that that's where they are.

- Male, 4-10 years service, Alpha Jail.

Notwithstanding this seemingly simple set of 'basics', with frequent contradictory expectations and instructions and delays in formal training, it was difficult for a probationary officer to know what to do or how to react when difficult situations arose. In the following example, the probationary officer thought the appropriate response was to deal with the situation herself immediately. However, the value placed on security by officers, of which the control of one's radio played a vital part, was higher than any potential incident or harm which may result. As such, her response was seen as incorrect:

Yeah it is hard knowing what to do.... I remember this one time (physical confrontation between two inmates), it was over turning the washing machine off because one guy (inmate) couldn't hear what he was saying on the telephone. Being new in the job, I put everything down on the floor, including my radio. I pulled them apart, locked one up, locked the other up. The officers came up the wing 'what have I said to you, you call for assistance' ' well I couldn't, I thought I was doing the right thing' 'well you weren't, you get us'. You know? And then I had to go back and find my radio - they (officers) gave me heaps about that. But I really thought I was doing the right thing.

- Female, 4-10 years service, Alpha Jail.

As indicated in this extract, officers would remind the errant officer of their mistake and recount this situation as instruction to future officers - one of the many ways in which the probationary officer began to became aware that their 'self' was no longer private and that their mistakes - their inability to get the basics right in some capacity or other-could become part of the prison folklore (see Goffman, 1961). 
Many longer serving officers reported that the best advice they received when starting was to 'shut up, listen, and learn until they got their bar up':

\begin{abstract}
When I started my unit manager at the time says 'so what have you learnt today, what have you seen?' And I said 'this, this and this'. And he says 'all right, that's good'. They (Unit Manager) had a (induction) booklet they have to go through, and he closes the booklet and he says 'I'll tell you one thing', I said 'what's that?' He said 'once you start you're on probation for twelve months, and in that twelve months you shut your mouth, you follow the orders that you're told. If somebody (inmate) asks you a question you don't answer it. You say nothing. You don't answer anyone (officers) back. You just shut up and do what you're told'. He said 'once you've got your bar up, after the first twelve months, then you can answer people's questions'. That was the best advice I ever got.
\end{abstract}

- Male, 4-10 years service, Beta Jail.

This officer was fortunate as a Unit Manager experienced in the traditional prison officers' script instructed him on how to learn and survive - this was often not the case now with probationary officers being ‘trained' by inexperienced peers.

Notwithstanding this lack of adequate guidance, the perception held by many longer serving officers was that probationary officers were increasingly challenging this aspect of the traditional script by questioning processes, systems and orders. They simply would not 'shut up and listen':

I think they're employing a lot of useless staff. Like from the days when I started, which is not that long ago, in the first year you do what you're told and don't ask questions unless you're not coping with something. And after you get your bar, after a year, you still do your job but then you can start questioning stuff. But for the first year you just learn. Now a lot of new staff have education, used to be in Uni, or a manager, or in good jobs. And as soon as they come on the floor they think they know everything?! You actually have to be on the job for a while before you realise how those people (inmates) think, how you affect inmates and officers and their relationships, you know. It's an environment which you (probationary officers) know nothing about. And these new guys just come in and charge everybody (inmates) who does something wrong. That's not the way we (experienced officers) do things, it makes the job more dangerous.

- Male, 0-4 years service, Beta Jail.

With the high level of uncertainty which now abounded as to how to perform the job, experienced officers proffered the warning for probationary officers to simply 'never say yes'. Such insightful advice acknowledged the ambiguity experienced by the 
probationary officer and incorporated an important element of the prison culture whereby an individual had to deliver on their word:

\begin{abstract}
When you don't know what to do there is only one thing to do - just never say yes until you know what you're talking about. That's (saying yes) fraught with danger. Once you say yes it has to happen but you can change a no into a yes.

- Male, 4-10 years service, Alpha Jail.
\end{abstract}

Similarly, experienced officers let probationary officers know that some inmate offences were 'acceptable' or 'tolerable' as opposed to those requiring discipline, formal or otherwise. This feature of the traditional script still imprinted itself on all the new variations of it that one could now find in the prison. It was only by tolerating minor rule violations or permitting aspects of the inmates' 'underlife' behaviours that officers could secure compliance on the more central issues of the prison regime (see Goffman, 1961; Sykes, 1958). For example, there were occasions when it was prudent to allow inmate justice to take effect. If an inmate had infringed on another inmate, retribution was compulsory within inmate culture. An experienced officer would permit a just deserts model of retribution (inmate initiated punishment in proportion to the crime) whereas an inexperienced officer may put the offending inmate 'on report'. Putting the inmate on report removed the inmate from the unit temporarily and could prevent inmate justice occurring in the interim. However, inmate culture demanded that retribution would occur and the temporary removal of the inmate only put this process on hold. Without officer 'guidance' the retribution could escalate to unreasonable violence against the offending inmate. Therefore, while the inexperienced officer felt that they had prevented a potential incident by acting 'by the book', they might have brought about an intensified response:

Yeah, there are rules upon rules in here. If you fuck up here you're going to fuck up majorly. You (officers) better get on board and their (inmates) system depends on it. It's a very complicated fucking system and it's not taught at college. It's like turning a blind eye to the inmate justice system when someone gets the shit kicked out of them. It's a different thing when someone gets a crack for bloody stealing something than if four people fucking try to kill somebody. I mean you should fucking know why it's happened. If he's cracked because he's an arsehole or stealing deodorant or something like that, well that puts a different perspective on it. That's when you turn a blind eye. I mean usually you know before it happens, but they'll crack when no one's looking. Most of the time you can work out whose handiwork it was, especially if you've been in the place for a length of time. They know that if he just gets moved out he was going to get 
fuck all and their system depends on retribution. It’s a very complicated fucking thing.

- Male, 4-10 years service, Beta Jail.

As this extract demonstrates, even the inmates' code had 'rules upon rules' and would be complicated for a probationary officer to learn. Further, the inmates' code required acceptance of aspects of the traditional officers' script that many new officers were unwilling to adopt - turning a 'blind eye' to violence, as this was not what had attracted them to the job. Such subtleties of rules and potential severity of consequences could cause significant stress and anxiety for a probationary officer. Therefore, not only did they have to learn the multiplicity of available officer scripts, but they were also required to quickly learn and accept the inmates' script.

Learning how to communicate within the prison environment was another major task that required significant attention in the first few months so that probationary officers could understand what was happening around them and also make themselves understood - the prison had its own language. Misunderstandings as a result of misinterpretations between officer and inmate argot sometimes arose. For example, inmates would use the derogatory term 'screw' to refer to an officer, though typically they would use this term when they thought the officer could not hear. If an inmate called an officer a screw directly, it was generally in a joking context or when the inmate was extremely frustrated with a process. However, newer officers were frequently offended by such language and sought to charge the inmate. Senior officers would not take this action, though, as they were aware that in such circumstances it was not a personal affront and the traditional script allowed for such indiscretions. Officers would only refer to each other as a screw if they were being jovial. As Goffman (1968) argued, such references to each other demonstrated a 'knowing' of their stigma attributes and functioned to enhance group solidarity.

Officers would call inmates a variety of derogatory terms when the inmates could not hear or in a joking tone with them, ranging from 'crims' to 'scrot-bags'. Longer 
service officers would use slightly different terms such as 'boob-head'139 for an inmate who had been in the system for a considerable period of time. In this way, the use of language could enhance group solidarity amongst officers while also fostering the impression of amicable, relaxed relations with inmates (Goffman, 1959). As per the understandings of the new Head Office script, with few exceptions officers would call inmates by their first name. However, they reverted back to the understandings of the traditional script when ordering them to perform a task, when they used the inmate's surname. Importantly, this indicates that there were at least a few elements of this new script, and of the traditional script, that all officers were required to attend to under the new working arrangements. Such subtleties of language use were significant and officers needed to know which script to use. If an officer were to call an inmate by their first name while ordering them to fulfil a task, the inmate might belligerently assert 'I'm not your mate' and refuse to comply with the request, indicating a breach of the language code and a misunderstanding of the officer-inmate relationship on the part of the officer.

\section{(ii) Style}

Similar to Goffman's (idem) primacy of personal front, appearance and manner for impression management in everyday life, officers suggested probationary officers needed to develop a style, a way of being, in the prison environment. Therefore, one of the most fundamental skills, at least according to officer culture and irrespective of which script one adhered to, was how one carried oneself. A new recruit was easily identifiable by their slouched 'civilian' posture and an uncertainty of what to do with their hands, often swinging them to their sides as inmates do, or how to authoritatively lean against objects (frequently readjusting their poses). Officers, on the other hand, were easily distinguishable by their straight backs, shoulder width placed feet, confident placement of their hands (clasped behind their backs, or often skilfully swinging their keys), and fixed poise. New recruits and probationary officers were more similar to inmates’ bearing than to officers. Longer serving officers, however, were discernible from inmates in a variety of easily observable ways. For example,

\footnotetext{
139 An old argot term for the prison was 'boob'. Therefore, a boob-head was someone that lived in prison (typically an inmate but could be used in reference to a long serving officer).
} 
when standing near a wire fence, inmates frequently interweaved their fingers through the wire (above head height). These officers, on the other hand, would never touch the wire unless a task required this. These subtleties of bearing had to be learnt quickly by the probationary officer and be adopted as a 'natural' way of being if one were to be 'seen' to be an officer.

How one carried oneself also incorporated the realisation that the uniform did not 'make the man'. Thus the probationary officer needed to establish a position of respected authority, be aware of their limitations and handle situations with considered responses rather than relying on physical dominance:

It's not about being staunch, it's being able to handle a situation, you know. You won't staunch your way through sixty inmates! No way you'll do it. Doesn't matter if you've got ten black belts, the fourth one (inmate) will get you. You're only one person. Intimidation doesn't work and these guys have seen it all. They were beaten as kids, its just water off a ducks back to them.

- Male, 4-10 years service, Alpha Jail.

Importantly, as argued by Goffman (idem), fronts are not actually created. Rather, individuals select from pre-established options - in this case, options that derived from the traditional officers' script. What became problematic, however, was that the style options became unclear with the multiplicity of scripts, making selection of appropriate fronts by officers increasingly difficult and likely to lead to inconsistency and unpredictability - regarded as the most unwanted features of prison life. Every aspect of this, including its people, had to be routinised and known. Therefore, there were few objections if someone was consistently efficient, fair, strict, incompetent, whatever. Objections arose at any hint of irregularity, positive or negative, whether it meant dinner was five minutes late or an officer who generally said 'no' suddenly said 'yes'. Hence the general opinion:

I know it's easier said than done, but you've got to be consistent. There are times when I really hate having to confront a big tough inmate with an assaultive (sic) history, with a reputation where it would be much easier to back down. But then when you're stressed you almost relish the prospect at yelling out at some small 75 kilo inmate who's always gobbing off to staff and there's no risk to your physical well-being to do that. Are you going to do the same to some big burly inmate who's going to yell out abuse? You've got to maintain the consistency. It's tempting, it's natural, people don't want to get into confrontations with 
people that are physically capable of ripping their arms off but that's part and parcel of being a prison officer if that's your style. \{my emphasis

- Male, 4-10 years service, Alpha Jail.

Attempting to be consistent could place considerable stress on the probationary officer until they developed their own style:

The difference is that on the outside you'd act without thinking, you know. But on the inside you're consciously thinking 'am I being consistent? Am I appearing to back down?' You're checking yourself all the time, or at least I find myself doing that and it's hard work. 'Am I being too friendly to the inmates? Am I being too distant?' Or 'am I trying to be too tough?' You know? You're just conscious of how you're acting all the time, like you haven't got enough to worry about!

- Male, 0-4 years service, Beta Jail.

Being cognisant of the front that they were fostering was important, however, as the audience (officers and inmates) constantly monitored the authenticity and veracity of the various cues to establish if there was any misinformation, deceit or feigning on the part of the performer (idem). If such a slip was detected, successful interaction broke down. This would typically manifest itself as a confrontation between the inmate and officer or insubordinate responses by the inmate. It was imperative, then, that probationary officers developed a style and showed it to be consistent across space and time that was able to maintain the difference between themselves and the prisoners.

\section{(iii) Loyalty}

Regardless of which version of the script one primarily adhered to, in the first instance what was required from all officers was loyalty to their other unit staff. In this way, it was necessary for each officer to rely on other officers to behave appropriately within the prison context, thereby creating a 'bond of reciprocal dependence' (ibid, p. 71). Thus: 
that will protect you. They're the ones that will cover your back. Learn that straight up or you won't be here long.

- Female, 4-10 years service, Alpha Jail.

At the same time, the probationary officer was warned against developing specific affiliations with particular staff members until they were aware of the group dynamics and until they had established their own way of being in the prison environment:

Officer: Don't be unsociable, but don't go buddy-buddy with certain people, you know. Just treat everybody as if they're the same.

Interviewer: In terms of inmates or the officers?

Officer: Inmates and officers! Don't just go buddy-buddy with one certain officer because he treats you nice and makes you, you know. Just treat all the officers the same until you know who's who around here. You go buddy-buddy with the wrong person and, well - you know!

Interviewer: Who's the wrong person?

Officer: That depends (laugh) but yeah, once you're in with some officers (particular groups or cliques) we'll never, well - you know!

- Male, 4-10 years service, Beta Jail.

This particular extract draws attention to the consequences of what was left unsaid in the use of the colloquial 'you know'. The implication was that appropriate ways of being should be known, but because of their complexities new or probationary officers would probably be left in the dark by these oblique references.

The requirement to be 'sociable' but not go 'buddy-buddy' placed the probationary officer in the difficult position of not knowing with whom to align while being required to 'fit in' with the entire team within the unit. The safest tactic for the probationary officer to employ was to align with their induction officer and their group. If the relationship later failed, the probationary officer could simply blame the association on the administration. In most instances, however, officers continued to remain associated with their induction officer's group.

The complexity of the informal rules and values which needed to be applied in order to be part of the team made fitting in with one's fellow officers increasingly difficult in contrast to the much more straightforward fit there had been before restructuring. For example, the majority of recruits came from blue-collar backgrounds where there 
was an established ethos that one started and finished one's shift at specified times. Within the prison, specified duty hours were formally applied. However, prison officer culture directed officers to start a half hour earlier and not be in a hurry to leave at the end of shift (see p. 32). It was asserted by officers that this demonstrated a willingness to relieve the officer on shift and a desire to be sociable with officers after the shift. If a probationary officer failed to detect this requirement or their induction officer had not brought it to their attention, as was frequently the case given the inducting officer themselves often had limited experience, the probationary officer could very quickly became ostracised from the team they were meant to be part of.

The second aspect of loyalty that the probationary officer needed to acquire quickly was loyalty to the officer group as a whole. They always had to backup an officer and never inform ('nark') on an officer. If one had an issue with a fellow officer, one was expected to always deal face-to-face with the given officer ('front up'), but at the same time, they were never to make an officer look bad in front of non-officers. Officers were so 'taken in' by their required performance of control in the dangerous environment of the prison, according to the traditional officers' script, that the threat of a team mate compromising the performance was too much to bear. Always backing up an officer was important for the group to feel safe and secure. That is, having faith that one's colleague would remain by one's side and provide whatever support was necessary during times of trouble. Leaving a problematic situation ('doing a runner') or being unavailable (such as having one's radio turned off) was deemed the worst behaviour an officer could display:

I think that you've got to be loyal to the guys in green (officers). Just as long as you're prepared to back them up, that's probably the biggest one eh, just be prepared to back your mates up all the time.

- Male, 4-10 years service, Beta Jail.

Always backing up an officer was to supersede any allegiances or disharmony that may exist between units, social groups and so on:

I think its like 'looking after your own', eh. I find that more so in a violent situation. Even though we may not get on with the person, we tend to stand up for each other because at the end of the day if you need someone to cover your back, and that person's in the wing, whether or not that's who you'd want to be 
there or not, you'd want them to be there for you, you know. Like we can all fool around together and we can, well I have ripped into staff for not doing what they're told, but at the end of the day I expect them to back me. You look after your own no matter what. \{my emphasis

- Female, 4-10 years service, Alpha Jail.

Similarly, the probationary officer needed to learn the rules against 'narking' and 'papering' quickly as these behaviours could permanently exclude the 'offending' officer from the wider officer group. 'Narking' involved any officer telling anybody outside of the officer group business that related to any action, thought, or circumstance of an officer inside (or outside) of the prison. As will be shown further in this thesis (see chapter six), information, especially pertaining to fellow officers, was a valuable commodity, and therefore was not to be divulged outside of the group. To do so, particularly to management, not only weakened the value of this information, but also jeopardised the officers' perception of their day-to-day control of prison events. Thus:

\begin{abstract}
You just don't nark! Usually, if you close ranks they (errant officer) tend to fix it (errant ways). Whereas if you blow it out of the water, well, that's that officer gone (errant officer's employment terminated). And that reputation (as a nark) gets carried throughout the job, you know. It's just like there's an unwritten rule among officers that you don't go nark on each other. Might be you that does something stupid next. But there's a limit eh (to how far you cover up). I can think of three instances off my head where they've (officers) covered up and I wouldn't do that. That's devious. I mean there are some shocking things that they've covered when they shouldn't have. But there's a difference eh, I won't lie, I won't cover up for nobody, but I won't nark neither. I just won't say nothing.
\end{abstract}

$$
\text { - Female, 4-10 years service, Alpha Jail. }
$$

However, there now appeared to be a difference in the application of this rule according to an officers' length of service. Officers who had served more than four years were vehemently against narking, but some of the newer officers were more inclined to put issues on paper, even though the experienced officers had warned them against the practice. This placed all officers with less than four years service under suspicion by longer serving officers, which decreased the likelihood of their acceptance within the wider officer group:

We tell them but some of them don't listen. What they don't realise is that there are a lot of implications to that and although you need to cover your backside, 
there still is that group thing. Yes you've got to cover your butt yourself, but if you get in the shit the group might close in and look after you and help you. If you're one of those people that gets a kick out of writing incident reports you'll find that the group will go away because they don't want to be with you - not a good way of being.

- Male, 4-10 years service, Alpha Jail.

Overall, then, learning the informal rules had become increasingly complicated as probationary officers were paired with inexperienced induction officers and hence, were not being instructed effectively in the traditional officers' script which explicated these. Further, as these new style officers were being attracted to the prison service by the prospect of excitement and challenge and thus the new Head Office script, there were aspects of the traditional script that they were simply not prepared to accept. Their subsequent behaviour was then judged by longer serving officers as 'inadequate' as they were not 'getting the basics right' nor developing the appropriate style or displays of loyalty. Paradoxically, this made the requirement to 'test' new officers more important to the officer group as they were all 'under suspicion', but at the same time, due to the multiplicity of scripts, this was more difficult to achieve.

\section{Testing And The Consequences Of 'Incorrect' Behaviour}

Established officers emphasised to the probationary officer that they should be aware that 'everybody is watching you'. This awareness was pivotal to displaying the appropriate behaviour to earn respect from inmates and trust from officers. While, in many jobs, collegiality and reciprocity of behaviour may not be vital to achieve work objectives, within the prison system it was essential to establish negotiated authority to 'get the job done' and be considered a 'good officer'.

When asked what made a good prison officer, officers reported that they and the inmates evaluated an officer on length of service and reputation (precisely the values that the prison authorities had devalued in the restructuring): 
It comes down to how long you've been here and how far up you are here. Like I can push an inmate to his limit. That's not a problem because I have a reputation here of being a woman who doesn't put up with any shit. Being a woman who's got a big mouth. Being a woman who's not scared to hit anybody. And being a woman who's not afraid to step into some inmate's room and say 'get rid of that fucking tape', you know. And it's not a problem for me to do that and that comes down to long service and reputation.

- Female, 4-10 years service, Alpha Jail.

Length of service required the passage of time, getting the bar up. However, attaining the appropriate reputation, or respect and trust, required suitably timed displays of behaviour. A common mistake of new and probationary officers lay in attempting to circumvent the means of attaining respect and trust within the prison environment with incorrectly timed behaviour:

\begin{abstract}
You have to earn respect in this place. It doesn't come on a plate. It's something that new staff find very difficult to understand. They come in and see the way older (more senior) staff talk to inmates and think they can do the same but they can't. They haven't yet earned the right to speak like that or the respect. And in the end what happens is they end up getting a whack in the face by the inmates or they have more difficulty getting tasks accomplished because the inmates just think they're cocky or smart-arses.
\end{abstract}

- Female, 4-10 years service, Alpha Jail.

A second common mistake of new and probationary officers was in overlooking the cooperative arrangements and mutual interdependence between officers and inmates. They needed to be aware that they were not merely developing a rapport with their colleagues, but also needed to fit into the inmates' framework:

\begin{abstract}
I'm a believer that respect wasn't just something you gained because you wore a green uniform. It wasn't just given, you earned it through the old code, if you get what I mean (respect through direct character testing). You get a lot of the young (new) staff come in and they think they're a prison officer and they'll jump around. We (officers) only run this place if they (inmates) let us. And they showed us that a while ago (Green Unit riot). And that was nothing (potential for worse). That's the reality of it.
\end{abstract}

- Male, 10+ years service, Alpha Jail.

The majority of probationary officers needed to consciously develop the requisite rapport with inmates. However, there were a few exceptions to this. Probationary 
officers who were either physically commanding, visibly 'brown' or were accepted promptly within an ethnic clique needed to make little effort to gain respect and trust:

Size has a lot to do with it. I mean we had an officer here who was only in the job for about two months and was built like a brick shit-house. I mean he had no bars up but the inmates sorta looked him up and down 'no ain't gonna take this guy on'. They pushed him a tad too far one day and boy they found out who was who (laugh).

- Female, 4-10 years service, Alpha Jail.

On the whole, however, the probationary officer was likely to face a series of character tests, which could be inmate or officer initiated. These tests were to be endured without complaint until the senior officers deemed one 'capable' or the probationary officer left the service at their prompting. The tests were designed to instil confidence in the particular probationary officer and let the officer group know that the probationary officer could be relied on, had become 'one of them':

\begin{abstract}
We can't really test them now. We did that more a few years ago when it was sort of the rule of the jungle and nothing was ever questioned. You know, if a crim played up you'd take him around the back and crack him, but nowadays it's so different. You send some of them out there to see how they handle it but if they fuck up it'll all be on. They don't have the people skills. They don't have the street smarts. You send them out there and you'll shatter him straight away. It's hard enough in here without that on your conscience.
\end{abstract}

- Male, 4-10 years service, Beta Jail.

Thus only minor testing remained. The primary reason for this was that the lack of homogeneity and unity within the officer group had increased the longer serving officers' suspicion and mistrust of the new style officer. There was now an uncertainty as to which script the probationary officer may be acting within and, thus, what their reaction may be to a direct character test. Hence, any testing done by the officer group needed to be both subtle, and 'justifiable' if questioned by management, such as requesting the enforcement a formal rule:

We still do some small stuff, like telling them to get something off an inmate, like a red t-shirt ${ }^{140}$. No one else would dare go and take it off them but we'll send the proby off to do it.

- Male, $10+$ years service, Beta Jail.

${ }^{140}$ Certain items of clothing were forbidden within the prison and the possession of such items was a
status symbol or trophy for inmates. Within the New Zealand context, one of the most prized items of 
Notwithstanding the minor testing, generally officers now merely observed the character testing performed by inmates. These tests had always occurred, however, and were not considered particularly interesting to officers until they became unable to conduct their own tests. Such tests typically ranged from minor transgressions or the pushing of boundaries, such as asking the probationary officer to supply an item disallowed by another officer, through to direct confrontations (both verbal and physical and ranging in intensities):

Yeah I got tested all right, you're being tested all the time at the start. These guys will take you to the limit if they can. It's just that testing phase. It's the uncertainty of what's going to happen next that gets you down. Even though it's only a mental thing, it sort of affects you physically as well. I used to go home at night and feel zapped, but now everyone knows how I'm going to be and all that testing has stopped.

- Male, 0-4 years service, Alpha Jail.

There were two outcomes of character testing. The probationary officer would behave appropriately and gain trust and respect, or their behaviour would be deemed inappropriate, making trust and respect, indeed survival in the job, harder to achieve. The following is an example of a new officer who did not behave according to the requirements of the traditional script:

\begin{abstract}
We all know what happened. [Probationary officer's name] went in there (kitchen) to do the muster and saw them (inmates) drinking (homebrew alcohol) and what did he do about it - nothing! ${ }^{141}$ He can't say he didn't see them because he mustered them ${ }^{142}$. He didn’t say nothing, bastard. I'll never forgive him for that. [Subsequently seriously assaulted officer's name] goes in there and saw. He tried to do the right thing, he called for assistance and we tried bringing them out but it was too late and the shit hit the fan (Green Unit riot ensued). [Subsequently seriously assaulted officer's name] gets the bash, the wing was trashed, fuck! [Probationary officer's name] should have been fired but he wasn’t even fingered
\end{abstract}

contraband was red clothing due to its association as the primary colour of the Mongrel Mob. In the New Zealand prison system this gang is typically very high in the inmate hierarchy and have significant influence over the inmate population. This gang is also one of the most visible and notorious gangs in New Zealand society generally (for more information on New Zealand gangs see Payne, 1991).

${ }^{141}$ The correct behaviour for the officer to have done was to inform the IC who would then organise C\&R teams to remove the inmates back to their cells and lock them down so they could not cause any problems (or apply some measure of informal techniques to remove the inmates from the kitchen back to their cells). The inmates would then be put on formal charge if deemed appropriate (or informal discipline measures would be instigated given the inmates having brewed alcohol reflected a breach in the officers security standards).

142 Once a muster was completed the mustering officer was required to sign the inmates as accounted for in the muster logbook held in the guardroom. Having signed for the muster indicated that the officer at least claims to have seen each inmate at the specified time. 
in the report. He lied ${ }^{143}$, that's about as low as it gets ${ }^{144}$. All they (management) did was move him (to another unit), but everyone knows. He won't last. Who's going to trust the bastard now?

- Male, 0-4 years service, Alpha Jail.

What is particularly interesting in this extract is that even though the probationary officer had severely offended the officer group, officers did not 'nark' to management as this would be contrary to the traditional officers' script. Instead, the informal means of disciplining officers was invoked whereby the group withdrew allegiance and support from him, making his job difficult to perform. Clearly, then, to establish trust from one's fellow officers (and concomitantly respect from inmates), regardless of which script the probationary officer or observing officers predominantly used, the traditional officers' script was dominant in both the expectations of behaviour and response from fellow officers.

In contrast, the following is an example of a probationary officer who displayed acceptable reputation enhancing behaviour. While the behaviour was not acceptable according to formal requirements, the probationary officer demonstrated to other officers that he was adhering to the traditional prison officers' script and that he would follow its instructions rather than those set out by Head Office in the new script:

Being the new boy on the block I knew nothing and everyone looks at you sideways... so my offsider (IC) says 'breakfast at 7, you muster everybody to get up and get their breakfast'. So I said 'what happens if they don't get up?' and he says 'drag em out'... well one person didn't come for breakfast and my offsider says 'go get him'. I walked into his cell, not knowing anything about the internal etiquette or what you do and don't do in an inmate's cell ${ }^{145}$ - so I just walked in and told him to get out of bed and he fucking nutted off, went absolutely ballistic. 'Don't fucking tell me what to do, my boy gets my breakfast' and I says 'you get your own fucking breakfast'. Well, he started 'oh fuck you screw',146

\footnotetext{
${ }^{143}$ The officer claimed not to have seen the inmates drinking alcohol. However, the other officers felt that given the state of intoxication when the second officer went in it was not possible for the first officer not to have noticed the problem.

${ }^{144}$ Lying, or being 'economical with the truth', was acceptable if it was to protect the group, but in this case the lie was to protect an individual at the expense of the group.

${ }^{145}$ Prison etiquette for entrance into a cell was to notify the inmate of one's presence (knock on the door) and ask for permission to enter. While it was formally acceptable for an officer to enter at any time, informal etiquette was paramount as this was the inmate's space. When this probationary officer simply walked into the cell without invitation he had breached the known understandings and it was unlikely at that point that he would gain any cooperation from the inmate.

${ }^{146}$ As a probationary officer, this officer would have taken offence at this label. However, it merely indicated the breach in cell etiquette.
} 
and I thought 'oh fuck, I'm going to die on my first day'. He turned over on his bed and faced me and oh my God - this guy was huge, six foot eight, skinhead, tattooed to the hilt all over his head, fuck I'd never seen a person that tattooed! He kept verballing me and I thought I was going to get smashed, so I thought 'I'm not going to wait to get hit'. So I dragged him off the bunk and smacked him right between the eyeballs and he fell out of the cell. I walked over the top of him into the wing and there were 56 inmates all lining up for breakfast looking at this guy... next minute the IC comes running down 'what are you doing?' 'You said if he didn't come down to breakfast to drag him out' 'you can't do that'.... I got taken into the boss's (Superintendent) office and got 'talked to' about it.... Turned out he (inmate) was the man of the wing, he had all the push, and I went in there and pinned him over! So that incident followed me around for the rest of my career, everyone thought I was mad but no one messes with me.

- Male, 4-10 years service, Beta Jail.

The most important consequence of not following the informal rules was the affect it had on one's reputation. The prison has a memory and any breach would follow that officer for the rest of their career:

In a case when I was papered for something, I was amazed at how the fact of what the (narking) officer had done had gone around the jail so quickly and it was much more in detriment to that officer than it ever would have been to me. So suddenly all the other staff were involved in it. And an officer came back to me and said that if an incident took place they (narking officer) might be required to take one or two more hit's than were necessary. And that's the way reputations are built or broken down. The officer that put me on paper, they'll have that hanging round their neck forever! There'll always be someone that remembers it eh.

- Male, 0-4 years service, Beta Jail.

While it may seem that the first year of service was a troublesome time for the probationary officer, the majority did survive and did get the bar up. Receiving the stripe to attach to their epaulette was typically marked with celebration by the transitioning officer's unit staff. Jovial 'pats on the back' and assertions of 'you're now one of us' accompanied the transition. Their new identity as an officer not only reflected a change in acceptance by the group, but also signified the change of their 'self' as they successfully adapted to the role expected of them (see Crawley, 2004; Goffman, 1961; Wicks, 1980; for family recognition of changes see Cheek \& Miller, 1983; Crawley):

Yeah it changes you to a certain degree. It's like you sorta get compassion. Like you see the casualties, they cut themselves up or whatever, and you learn not to loose all compassion just because they're inmates but you also sorta become non-reactive. Like if I saw that (self mutilator) before I come here it would be quite dramatic 
whereas now you just go deal with it... yeah, reacting to dramatic situations at a non-emotional level was a big change... when you go home you're less dramatic, you're sorta non-responsive to people's crisis's, you detach, I guess that's the word for it, you're wearing a bit of a mask or, you know, a shield or something, but at the same token you can't take it off. \{my emphasis\}

- Female, 0-4 years service, Alpha Jail.

\section{The Emergence Of A Prison Officer Self}

As this new self emerged, the new officer realised that they were no longer like those on the outside of prison. Their social bonds had loosened with that world but had strengthened with the world inside the prison. Indeed, new officers at this point came to realise that being an officer actually reduced their life in the outside world where they were now labelled as stigmatised 'outsiders':

To the world out there we're just like the dumb old prison officers. All brawn and no brains, only here to unlock the doors and drink coffee. I just don't bother. I'm not here to justify my existence or glamorise or build up their expectations about the prison. They can think what they want to think.

- Female, 4-10 years service, Alpha Jail.

I don't really like to say that I work in prison because immediately you say you're a prison officer they look at you as if you've grown another head or something. And then they come out with all these ridiculous things that they've seen, read or heard, and you spend the whole time thinking 'my God, why did I open my mouth?'

- Female, 0-4 years service, Alpha Jail.

As Goffman (1968, p. 24) noted, such self-isolation, while insulating the individual from the effects of their stigma from outsiders, could lead to the individual becoming “suspicious, depressed, hostile, anxious, and bewildered”. At the same time, they became increasingly cynical and suspicious of those beyond the prison, while at the same time strengthening their bonds within the prison:

Probably a few years back I wouldn't have thought twice if I saw people walking past a school, that's no big deal, it's just somebody out for a walk. But when you've been in working with these kind of people (inmates) the first thing you think of is the bad things that could happen (criminal intent).

- Male, 4-10 years service, Beta Jail. 
There was also the realisation that the changes to the self were permanent and now constituted part of who they were as people, making them prisoners within the job, as well as prison officers:

\begin{abstract}
I think we (officers) are all prisoners, even when we go outside. It's just the same as police, they associate together, the same as the army, they have a certain way about them. There are certain expectations of how you're supposed to be on the job, and it's very easy to say 'leave what you do on the inside at work and not bring it on the outside', but it's very hard to do. Whether you want to or not you're still going to bring it out. If you act like that for eight hours a day then you will act that way as a person. You'll act the same when you go to nightclubs and when you go to your family and your friends. Maybe not to the same extent, but always something will stay with you.
\end{abstract}

- Male, 0-4 years service, Beta Jail.

Overall, then, the consequences of the erosion of the homogeneity and unity of the officer group had indeed affected the training of probationary officers. As the substantive training occurred on the shopfloor, probationary officers were ineffectively being schooled in the traditional officers' script due to the inexperience of their inducting officer. The effect of this was two-fold. For the probationary officer, it created considerable confusion and stress as the appropriate front and behaviours were no longer clearly prescribed. For longer serving officers, the probationary officer's inability, or unwillingness, to adopt the traditional officers' script was perceived as further evidence of their unsuitability for the job and grounds for neither accepting nor trusting them, causing further divisions to the prison service. In these respects, 'getting the job done' had become more difficult for both groups of officers. 


\section{CHAPTER SIX: HANDLING YOUR LAG}

As the probationary officers experienced firsthand, in the aftermath of the 1990s restructuring, the script that they had to learn to perform their part in the prison performance was being rewritten in strange new ways. However, all officers had to adjust to this, whatever their length of service, in order that they might 'handle their lag' and 'get the job done':

There's an old adage here 'handle your lag', you know. You hear that a lot. 'Handle your lag', from officer to officer (laugh). \{my emphasis\}

- Male, 0-4 years service, Beta Jail.

Prison literature has frequent references to inmates having to survive the pains of their imprisonment (see Johnson \& Toch, 1982; Parisi, 1982), but few accounts give recognition to the pains experienced by prison officers in the course of their work. And yet, as one told me:

There are the (formal) rules and there are the (informal) grey areas. To survive here you had to learn the grey areas and I remember going and seeing the staff training officer here at the time. I was so confused, here I am learning the job, here are the rules and they're black and white but nothing that's done on a daily basis fits within the rules. And the answer I got back was 'handle it, that's the way it is and if you don't like it pack up your bags and there's the door. \{my emphasis\}

- Male, 4-10 years service, Alpha Jail.

In this way, prison officers had previously been able to buttress the pains of occupation that being employed in the prison brought with it. Now, however, the changes in the prison service, whilst having been written into their scripts, were beginning to add to their pains, making handling their lag an increasingly problematic and contentious matter. 
As this chapter will demonstrate, the effects of the altered prison performance did not just impinge on probationary and new officers. All officers had to adjust as elements of the new Head Office script had become a reality of the job and needed to be accommodated if they, too, were to handle their lag. While the line between officers and management had become clearer, the new Head Office script, with the requirement of case management, had increasingly blurred the line between officers and inmates. The lack of a discernible line required situational judgements by individual officers, many of whom, as shown in the previous chapter, were ill prepared for such variability through their inadequate training. As such, disciplining inmates and maintaining control through cooperative activity required rewriting as even this had become contestable in the post-restructuring prison. In addition, the injection of ethnicity to the prison service had rewritten the script in unexpected ways. The initial division soon relegated white officers as subordinates within the officer group, a status change which they did not enjoy but were unable to redress. Alternatively, while women had similarly been written into the script by Head office, they were not able to assert themselves into the dynamics of the prison with much effect. Instead, the traditional officers' script dominated their involvement, dividing, atomising and controlling them - making theirs a particularly difficult lag. Notwithstanding this, there was still one means that any officer could employ to gain status within the officer group, thereby improving their working experience by inserting themself into the 'information hierarchy'. Although this had been a feature of the traditional working arrangements, it had taken on a greater importance as even though formal means of attaining status had severely diminished with the flattening of the career structure, officers were able to establish and maintain informal respect with and gain status from their colleagues. In addition, keeping information to themselves rather than sharing this with management gave them the impression at least that they still had day-to-day control in the prison.

\section{Relationships Between Officers And Management}

One central element running through prison officer culture has always been the need to establish relationship lines between oneself and other actors in the prison. This line 
symbolised how far a relationship could extend and what breached 'levels of association' rules. Previously, the line between officers and immediate management was based on the respected authority that was attached to the position of rank. The relationship between officers and Head Office, however, was based on suspicion of this distant body.

Between officers and ranked positions, communication paths had been simple and transparent. The 'management' ranks were viewed as an extension of the prison officer ranks and their position was achieved through length of service and prowess on the shopfloor. This was respected within the officer culture. While aware that the 'management' ranks 'governed' officer activity, this authority was accepted as those who possessed it were assumed to have an understanding of what it was to be an officer and that they had earned their status. Higher rank staff ensured that they were on first name basis with all officers and inmates and that they were regularly seen 'walking the wings'. At the target institution, this arrangement was possible due to the small size of the facility and low rate of staff turnover. While one could interpret this regular contact as a means for 'management' to check up on staff, officers viewed this as their opportunity to demonstrate their skills. The Chief would praise them for having a clean wing and tidy, turned out inmates - the officers were doing their job, felt appreciated and most importantly felt proud of what they were achieving. Also, there was a purpose to this - by good performance they too could work their way up the ladder and maybe eventually 'walk the wings' themselves:

The Chief was part of the prison officer structure, he was at the top, but he was part of it. Managers, even if they were from the floor, they're not part of the prison officer structure. It's a different thing. Their structure is on top of our structure, not part of it. \{my emphasis\}

- Male, 10+ years service, Alpha Jail.

As this extract indicates, the previous Chiefs were merely an extension of the prison officer group. However, as the facility and personnel numbers rapidly expanded and, coupled with the increased bureaucratic demands on their time, Managers, in the aftermath of restructuring, were no longer seen on the shopfloor of the prison: 
The major change is undoubtedly the separation between the people on the top floor and the people dealing with the inmates. In the days gone by, superintendents, when I started, would tour the institution at least twice a day, walk through the wings, talk to the officers, and talk to the inmates. When there was 200 in here (double housed inmates) he (Superintendent) would walk through the wings and the compound. The big boss nowadays is nowhere to be seen. You'll never ever see the big boss walking around the compound and walking the wing, never ever.... My idea is the man who leads is in charge, not sitting in a bloody office where we never see him.

- Male, 10+ years service, Alpha Jail.

Officers interpreted this invisibility as management being disinterested in their performance - except when something went wrong: it was only at that point that they would suddenly become visible. In addition, it seemed to officers that management were increasingly preoccupied with the needs of inmates, such as programmes and ensuring inmates' rights were met. At the same time, because of the decentralisation and corporatisation of the New Zealand prison system, management seemed to be invariably preoccupied with budget issues which did not fit with the officers' interpretation of the needs of the day-to-day functioning of the prison. Worse still, management were thought to be undermining the authority of the staff by siding with inmates:

The gap between management and staff is getting wider. The managers are focusing more on the budget than the best interests of the staff and inmates. But it seems to be that inmates come before staff? Whereas it used to be the other way around. They seem to see the inmates' point of view rather than the staffs'. It's just wrong!

- Male, 0-4 years service, Alpha Jail.

As this extract demonstrates, there was a growing animosity between officers and management due to the perceived 'favouritism' of inmates over officers. Indeed, the line between officers and management had become more firmly drawn and more difficult to cross in the aftermath of the industrial dissent of the 1990s and ongoing internal investigations which increasingly made officers feel dispensable and undervalued:

Officer: There's a lot of hurt out there. I think a lot of officers just mark time really. Just doing the job, just marking time.

Interviewer: Where does that hurt come from? 
Officer: Being shafted! Just the way they (management) treat you. I'm a number, that's all I am at the end of the day. And that's made obvious to a number of prison officers that are here for a while. That's all you are and you're not indispensable, you know. But before, and okay, there's twice as many officers now, but at least they (Chiefs) knew our names, and that's different.

- Male, 4-10 years service, Alpha Jail.

Furthermore, rather than the line bearing some correspondence to rank position, it was now drawn between those who wore a uniform and those who did not. In this way, communication paths stopped at the Unit IC level whereas, previously, communication was accepted through to the Superintendent level as long as it passed through the appropriate ranks:

\begin{abstract}
If he (Unit Manager) doesn't have a uniform on he must be management. I mean, I'll talk to him but I've got that barrier. I can only tell him certain things, you know. IC, yes, sweet as, because he's got a uniform on and I can relate to him and he works with me. And he's (IC) the boss as far as I'm concerned. So I'll tell him anything he needs to know. But it's always that thing when it comes to anybody with suits. You always got that sort of barrier between (you).
\end{abstract}

- Male, 0-4 years service, Alpha Jail.

As will become clear further in this chapter, the transmission of information, or lack thereof, was an important element of the officers' occupational culture and became an important element in the way in which officers attempted to assert control over management. Having access to this information also gave rank and file officers a sense of superiority over the managers who were dependent on them for this. Because of the new philosophy to fill management positions from outside the prison service, managers were being appointed who had had no experience of prison work. Officers felt that if the manager had not been through the system then they just could not understand prison life. In which case there was no respected authority and no chance of establishing communication paths:

One guy we had here, he only lasted six months. The thing was, he was an absolute disaster waiting to happen. He had all these management qualifications and stuff. I said to him 'the thing is, a rack of t-shirts isn't going to attack you because one t-shirt's had a bad phone call or one t-shirt is going out with another t-shirt's girlfriend so they stab each other', you know. You have to snap out of that. People are so complex and there's 450 complex people here.

- Male, 4-10 years service, Alpha Jail. 
As intimated in this extract, there was an incident associated with this manager's 'inabilities' on the shop floor. Again, the prison has a memory and it only took one negative instance, such as this, to become folklore and undermine the entire 'nonprison officer' management group. A small proportion of upper management had been promoted through the system and therefore possessed at least some experience of being a prison officer. However, as these new managers had not necessarily earned their position, as they would have done under the rank structure, coupled with the change in attire from a 'uniform' to a 'suit' that now came with this elevation, these ex-officers were now on the other side of the 'line'. In this way, even those managers who had worked on the shopfloor were no longer considered to be competent or able to understand the viewpoint of prison officers once they had been promoted:

\begin{abstract}
Fuckwit's upstairs got no concept of reality I think. A lot of the people who work there have come from prison officers and when they get up there as far as I'm concerned they take off their head and put on cabbages because they forget where they came from and they forget how the system works and how the jail works. And I've seen guys go up there who are awesome prison officers who, you know, totally know the system. And (they) goes up there and gets on board with this management rationale and stuff like that. It's all the reports I've seen by these guys - is this the same fucking person who five years ago was a fucking prison officer?
\end{abstract}

- Male, 4-10 years service, Beta Jail.

To illustrate this relationship, an officer might have wanted to transfer a recalcitrant inmate to another prison to enhance harmony within their own prison. Such transfer applications were required to be approved by upper management. When such a request was denied, it seemed to the officer that their knowledge and experience had been ignored by a distant entity that was seemingly oblivious to issues associated with the given inmate. As multiple requests were unsupported, the division between officers and upper management was cemented and resentment from the shopfloor rose. For example, during the course of my research, officers repeatedly attempted to transfer a notorious 'patched' inmate ${ }^{147}$ out of the prison and out of the region. At an informal level, the inmate was dramatically affecting the social and behavioural dynamics within the inmate group throughout the prison, and officers were aware of several serious threats to security gained through their usual channels of information

\footnotetext{
${ }^{147}$ A patched inmate is a 'patched' member of the Mongrel Mob gang. To become a patched member of this gang required at least one previous prison sentence for a serious/ vicious rape or murder.
} 
which included other inmates and gang associates. As formal grounds for the transfer request, the inmate's classification was actually maximum security and therefore he should not have been at the target prison. It was not until two serious assaults occurred, one hospitalising an officer, followed several weeks later by a significant breach of prison security involving external gang associates, weapons and a damaged external perimeter fence, that upper management conceded to transfer the inmate back to the maximum security prison.

Several important implications flowed out of the altered relationship between officers and management. In the first instance, without respected authority it would be difficult for management to feel confident that their directives would be followed. With the invisibility of management, officers became suspicious of management's motives when they were seen about the institution. Given officers could rely on the absence of management on the whole, this permitted a degree of autonomy for staff and their behaviour was largely left unchecked. As communication and information flow was informally blocked at the Unit IC level, this allowed great variation in unit behaviour (see chapter seven). As a result, there were increasingly different approaches to the grey areas of the formal rules, alongside differing approaches to the informal rules of reciprocity and differing approaches to control by physical intimidation:

\begin{abstract}
You know it's supposed to be wherever you are, whatever prison, it should be the same. These units make their own by-laws, they're still guided by the basic principles of PPM but with all of the sidelines and the by-laws in each unit you'd be friggin lost with what's going on. They should be the same but they're not cos nobody's watching.
\end{abstract}

- Male, 10+ years service, Beta Jail.

At the same time, a corresponding anonymity that also came from the absence of management was not useful for ambitious officers who sought promotion or preferential job secondment - this depended on management. Those officers seeking such promotion had to make sure that they were 'noticed' by management. But in doing this, they had to act outside of the terms of the new Head office script which now kept management distant from officers. This might then lead to resentment from those officers who followed the script and kept their distance - but with no reward: 
You get some (officers), something little that happened in the unit and he goes straight to the boss, you know, having late night suppers with the boss and telling the boss about what we do in the unit... only doing it to make their own little empire. It pisses me off.

- Female, 4-10 years service, Alpha Jail.

As indicated in this extract, not only were such officers acting outside of the new script, but also outside the terms of the traditional officers' script which abhorred 'narking'. Such communication with management, however, was the exception. On the whole, officers actively avoided any potential interaction. For example, many officers would rather park in a different unit's car park if the only available space was next to a manager's vehicle in case they happened to be leaving at the same time. Similarly, officers would scan the car parks to determine which managers were on site and, knowing their routines, were then able to avoid contact.

\section{Relationships Between Officers And Inmates}

While the line between officers and management had become sharper and more divisive, the line between officers and inmates had become more fluid and contingent. Previously, inmates were to be considered the antithetical other and not to be trusted. In certain respects, this still prevailed, particularly as regards two categories of offenders. The first were groups of inmates whose offences caused disgust amongst the officer group, such as child abusers. Officers acknowledged that this rigid line generally meant 'going by the book' in terms of providing only the minimum provisions, not spending much time with the inmate and generally preferring to deal with other offenders whom they deemed more worthy of attention:

I hate anything against the kids. I can't fathom those kind of people. Just defenceless little kids! I do what I have to do in my job as far as dealing with them. But as far as them getting anything from me that's when I go by the book. That's probably one of the few times that I'll go by the book and everything takes as long as it possibly can. They get 'no, no, no, no, no' until I'm told they have to get 'yes'. It's a shit way to do it but it's just how I think I can deal with them. You know, 'I'll fix you, I'll make your lag hard, I'll make you hate me' and that's just the way I go with it. Right or wrong it's one way of dealing with them.

- Male, 4-10 years service, Beta Jail. 
The second category of 'undesirable' inmates was those who offended against the 'status difference' between officers and inmates. Often it was not the criminal history of these inmates per se that placed them within this category but, instead, the attitude that they displayed toward the officer group. Typically the 'bad attitude' inmates were White Supremacist and white-collar offenders. Both of these groups treated officers as their inferiors and would not adhere to the informal rule sets of the prison denoting subordinates and superordinates:

I have a big problem with trying to deal with Asian fellas because of their arrogance towards any culture. Oh, and skinheads, I think a lot of us have problems dealing with the skinheads (pause). Fraudsters - smug fucks. It comes down to attitude eh.

- Female, 4-10 years service, Alpha Jail.

The previous clarity of the us-them relationship had made disciplining recalcitrant inmates unproblematic. However, with the introduction of case management and the increasing attention given to the rights of inmates, relationships between officers and inmates became increasingly unclear due to the higher level of intimacy that these 'patron relations' now required (see Goffman, 1961). There had always been patron relations - relationships between officers and inmates directly in their charge-but these were formally prescribed with clear boundaries. However, the previous distance between them was no longer effective to 'get case management done'. Officers were now required to work collaboratively with inmates towards rehabilitation. Working in such a way required the establishment of more consequential relationships between a case officer and their charges. In this way, officers and inmates became increasingly enmeshed, making the informal requirements to elicit trust from fellow officers and respect from the inmates incompatible at times:

There was a big change of concept with case management. Like you become more a buddy (to inmates), you know what I mean. I think that's what the downfall is because you can't be a buddy to some and be consistent.... In the old system you treated everyone the same because there wasn't that tie there.... You can't be close to a person and not feel sorry for a person, compassionate to certain areas. You get linked to their (case inmate) family.... Before, that closeness never existed. You had a loyalty between the inmates and the staff and a closeness which helped with the loyalty. But (it) wasn't what it is now where you deal with them, and became part of their family.

- Male, 10+ years service, Alpha Jail. 
Goffman (idem) argued that in total institutions, staff and inmates conceive of the other in stereotypical terms which seldom leads to sympathetic identification - yet, as indicated in this extract, this was precisely what was occurring. As Goffman (ibid, p. 89) noted: "[w]hen unusual intimacies and relationships do occur across the staffinmate line... all kinds of awkward reverberations are likely to occur...”. Unusual intimacies and reduced social distance was precisely what was required with case management. The awkward reverberations continued to be experienced by officers during the course of this research as unanchored social encounters given officers were frequently battling with where the appropriate line should be as it was no longer clear or prescribed. Indeed, an undefined closeness was now formally prescribed within the new Head Office script.

Situational judgements as to where to place the dividing line necessarily produced inconsistency which was the feature of prison life which generated the most negative reactions from both inmates and officers and, of course, was in complete contrast to one of the overriding rules the probationary officers learned in their various scripts. Now, however, it was up to the officer to work out when to bend rules to secure a working relationship with inmates - and how they could do this consistently:

It's up to you as to how close they (inmates) become, you know. Two officers and sixty inmates - it's not a situation that you will be able to control so you need inmates to be there for you. So you let some get closer to you than any other inmates so if a situation arises and you have no help from officers there is always someone there that you think is going to help you.... If they ask you for something small, like a visitor and talk to the Mrs., I can give them a telephone call. But if they're going to ask me for other stuff, it all depends, you know. You just decide it yourself, you know, it's not worth it to lose a job for an inmate. You draw the line.

- Male, 0-4 years service, Beta Jail.

As this extract demonstrates, the change in social relations necessitated cooperation from inmates. This became part of an economic and social exchange. With few authorised incentives, officers were now expected to offer indulgences (treats, extra phone calls and so on) or social favour (lessened disciplinary charges, favoured assignments and so on) in exchange for their inmates' assistance, be that for control in the unit or fulfilment of case management assignments. 
However, as probationary officers were increasingly paired with inexperienced induction officers, and were only exposed to 'the way of their unit', it was problematic to expect these officers to know where to draw their line. In such uncertain circumstances, individuals tended to employ strategies for protecting 'the self' (see ibid, 1959). Many officers thus reported that they had established an 'intransigent line', even if this seemed to go against the demands and ethos of case management.

While some officers did take the view that the new flexibility of officer-inmate relationships was a positive development, the majority thought that this only encouraged inmates to actively manipulate it. In this way, the loosening of relational barriers was not equipping inmates for the non-deviant life case management was intended to achieve. Instead, it only encouraged deceitful behaviour:

\begin{abstract}
Some of these guys (inmates) I quite like and there'd be a lot of officers in here that like certain inmates. That doesn't mean you trust them $100 \%$ or anything. You can never really trust an inmate. They're in here for cocking up! A lot of them will always be looking for ways to take advantage of you in anything, any weakness in a staff member. That's where some of these new guys (officers) have gotten into trouble. You can like them (inmates) but they're not your mates.

- Male, 0-4 years service, Alpha Jail.
\end{abstract}

As this extract reflects, officers were highly cognisant of the 'sympathetic identification' they had developed for many inmates. However, it was this heightened 'awareness' that kept the requirement of $a$ line uppermost. Drawing the line between officers and inmates became even more problematic with the increasing overlap between the officer and inmate cultures. Officers learnt very quickly that the most effective way to get things done was to apply the inmates' understanding of the environment. On such occasions, the two cultures acted in unison to create harmony and known ways of being, even though this made boundary lines more unclear:

I think officers appreciate inmates' values, whether or not they like them, they accept it as part of the daily routine, that it's probably bigger than they are. Like I've never seen officers say 'right, that's it, I don't want to see anymore bashings over a chocolate bar' - try stopping that one eh (laugh). But that's just a small example of how it's different in here, you know. In here everything is futile. I don't know. No one would like to think that you start thinking like an inmate, but I'd certainly like to think I at least appreciate it.

- Male, 0-4 years service, Beta Jail. 
While it might be expected that the behaviour and language of inmates and prison officers would reflect the difference in their social positions, what began to happen was that officers reflected some inmate values and practices. This was reflected in the tone and content of humour and some aspects of visual appearance such as hairstyle and 'civvies' clothing choice:

\begin{abstract}
There's a mirroring eh, now that's something that I've noticed over the years in working here. I say look around you. When I started here inmates had long hair all right, staff had long hair. The inmates shaved their hair off and they grew tails, next thing - staff have tails, inmates get earrings - staff get earrings, inmates shave their heads - staff shave their heads. It's cracked me up over the years.
\end{abstract}

- Female, 4-10 years service, Alpha Jail.

But it was particularly noticeable in language use. Swear words could pepper almost any sentence as we see in the following example, relating to an officer's reaction to an inmate he had disallowed a phone call being given permission to do so by another officer:

The fucking cunt! Worthless piece of fucking shit! Fucking go behind my fucking back, I don't fucking think so! (Leaves guardroom and sees inmate across compound ${ }^{148}$ ). Oi! Get your fucking arse over here! Don’t fucking walk away cunt. Oi! (Approaching inmate). What the fuck did I tell you? You get your fucking phone call when the fucking messmen have fucking finished! I'm not your fucking babysitter. I'm not here to fucken run around after you shit-bags. I've got shit to do. [Inmate's response]. Bullshit! Don't fucking give me that shit. You know the drill, now fuck off. I don't want to fucking see you for the rest of my God damn shift. [Inmate's response]. I'm warning you, you've pissed me right off, now fuck off! (Turns to leave). You'll get fucking nothing now.

- Journal extract, Male, 4-10 years service, Beta Jail.

Amongst some officers, the constant bad language, on top of all the other pains of employment, made them uncomfortable with what the prison had 'made' them become (see Cohen \& Taylor, 1972). At the same time, as with the emergence of a new prison self, one could not simply 'turn off' this language. In this way, their language created a barrier between themselves and the world beyond the prison. Many officers recounted stories to me of embarrassing incidents with outsiders due to the frequency of swear words, or where prison terms, such as 'kiddie fucker', were used

\footnotetext{
${ }^{148}$ Exact series of behaviours edited for brevity.
} 
as this had become automatic terminology for them but roused noticeable disdain in their audience. This contributed to their feelings of shame and stigma and further bound them together, making contact with 'normals' more difficult (see Goffman, 1968). Thus:

\begin{abstract}
My speech is disgusting. When I came here I had beautiful English but no one could understand me and I was forever being like a dictionary, giving out word meanings. You have to think simple. My language now is disgusting and I notice it more out of the work environment. I tend to talk pigeon English, I use very slang type words. Your language is just swear words and prison words. But it becomes like that because in this sort of environment it's the acceptable language.
\end{abstract}

- Female, 4-10 years service, Alpha Jail.

Overall, then, the lines between officers and management, and between officers and inmates had been rewritten in strange new ways by the policy changes of the 1990s. While their relationship with management had become clearer and more divisive, their relationship with inmates had become fluid and contingent effectively increasing their pains of occupation and making it an increasingly difficult lag. With neither formal nor informal workable prescriptions, no one seemed to know their place in this world anymore.

\title{
The Contestable Lines of Control
}

While individualised control (of specific inmates) may have required alteration within the new arrangements, rules of reciprocity had long been the central means of negotiating generalised control with inmates. This remained largely the same. The 'items for exchange’ may have changed over time ${ }^{149}$, but the culturally prescribed premise had not - if an officer did a favour for an inmate the inmate must repay it and vice versa. Similarly, social indulgence or favour must be seen to be returned. Officers used this technique in two ways. The first was to provide a generally tolerable environment whereby if one or more inmates were not well behaved the 'unofficial privileges' were removed for all. This ensured inmates themselves

\footnotetext{
${ }^{149}$ With the introduction of self-selected purchases of canteen items, and broadening of products available, officers needed to be more creative with the 'treats' they offered.
} 
enforced 'harmony' in the unit. The most common mode of exchange officers provided was in the form of blockbuster movies which were cabled through to the inmates' cell televisions to break the boredom of evening lockdown. The second technique was to provide privileges to specific inmates, such as turning a blind eye to property possession above the formally allowed maximum level, or providing otherwise unavailable consumable treats such as biscuits, ice cream or brand-name cigarettes. These rules of reciprocity thus explained such behaviours as inmates defending officers during inmate-officer altercations:

I told an inmate to sweep the floor and he come along with the broom and just whacked me on the back of my shoulder, you know. And I thought 'ah someone's actually done it', you know. But before I could turn around and call for assistance the Sa' ${ }^{150}$ inmates actually come over and said 'nah we'll deal with it' and there it was. I mean, I get on quite well with the Sa'as, the old boobheads, you know. I think they're actually looking out for each other because, you know, one day we might have done a favour for that particular inmate and he'll come back to us and say 'well your favour has been returned, see you later', you know.

- Female, 4-10 years service, Alpha Jail.

While the rules of reciprocity for generalised control had not changed, the close relationships required for case management could make disciplining individual inmates more difficult. There were occasions when officers had to be prepared to step back and display an authoritarian side if an inmate crossed the line or breached the rules:

It's (drawing the line) probably difficult when you first start in the job but you learn to harden up to the extent that you can be compassionate but then at the end of the day you still have a job to do. If a guy becomes a loose cannon and stuff, causing trouble, you have to be able to lock him down. It's like this last weekend I had a chat to two inmates over in the unit, one was having a problem with his Mrs. and has been playing up in the unit a bit so we had a chat.... now I can do considerate and compassionate, but at the end of the day if he throws a wobbly I've got no hesitation in locking him down or if I had to - taking him out.

- Male, 0-4 years service, Alpha Jail.

The formal means of instigating disciplinary proceedings against inmates had not changed. The officer was required to place the inmate 'on report' for a breach of

150 Pacific Island people, inmates and officers, were referred to as Sa'as. While 'Sa'a' is an abbreviation of 'Samoan', this term was not limited to Samoans in the prison context but included all Pacific Island nations. 
regulations. The Manager Custody or Visiting Justice would then hear the charge and come to a verdict at a later date. If the inmate was found guilty, a formal punishment was imposed. However, two informal changes had occurred in this process. In the first instance, disciplining inmates within this new arrangement could lead to divisions between officers. For example, some officers would place pressure on other officers not to formally report the rule breaking of their 'case inmates' as it might jeopardise parole applications, thereby reflecting negatively on the case officer:

\begin{abstract}
Say inmate [name] does something wrong, but he can't get touched because 'oh he's my man'. The case management officers, they get protective of their inmates. Case management in this institution was a downfall because the line didn't get blurred it got lost! The nice simple line of 'this is the inmates and this is us', and you have to maintain that if you're going to maintain control basically. And when there is no control you will have incidents like homebrew and staff assaults. We now have that week after week, because its not the staff who are controlling the prison, the inmates are. They're controlling the world, they're calling the shots. You're just the ambulance picking up the pieces. It's just not right. \{my emphasis\}
\end{abstract}

- Female, 4-10 years service, Alpha Jail.

As this extract indicates, with the introduction of case management, the line was becoming increasingly blurred with a cross-over of loyalties between fellow officers and between officers and inmates. The simple us-them split no longer applied.

Importantly, the patterns of behaviour which officers applied to inmates, such as reciprocity, also applied within the officer group - 'you leave my case inmates alone and I will leave yours alone'. This had implications for discipline and control and inmates manipulated officer relationships accordingly. For example, it was not uncommon during the course of my research to overhear an officer checking with the case officer how s/he would like to proceed with a discipline matter. Indeed, on one occasion I witnessed an officer tear up a written discipline report about his case inmate and threateningly assert that 'I'll take care of him' - referring to the officer who had written the report rather than his inmate.

Further, formal punishments were becoming increasingly rare, and when applied, seemed weak to the officers. While both the inmate culture and officer culture have always preferred informal modes of discipline to bring recalcitrant inmates back into 
line (see Hewitt, Poole, \& Regoli, 1984; McEleney, 1985), officers felt that considerable time and effort now went into formal charges required by the new Head Office script and the result was ineffective:

\begin{abstract}
It's like any of the charges we have to do, nine times out of ten all they're going to get is a smack on the hand. So you put a couple of hours of paperwork in what's the point of doing it? He might get five days in the pound, he's not going to think of it twice going for five days, five days is easy, you know. OPs (off privileges), that's no big deal, they just sleep most of the day anyway, it doesn't matter, you know.
\end{abstract}

- Male, 4-10 years service, Beta Jail.

While, in the past, officers felt supported by higher ranks when they submitted formal reports on inmates, they now reported a reluctance of the new structure of upper management to support the officers' charges because of the emphasis they gave to inmates' rights. Therefore, there was a growing reluctance by officers to charge under the new arrangements:

You're wasting your time charging them because the bosses are too lenient with them. I'll give you an excellent example here about how much rights the inmate has. An officer charges an inmate for swearing at him. That inmate goes to [Upper Manager's name] and spends for the best part of an hour and a half discussing his right to say 'fuck off' to an officer. It's true, an hour and a half about his rights to swear... he went on and on, got off scott free in the end. He talked his way out of the situation and that should never have happened, bloody hell, wasting your time.

- Male, 10+ years service, Beta Jail.

Hence officers felt justified in the use of informal discipline. Problems solved quickly by such means allowed a unit to return promptly to routine:

It's a judgement call, you think 'perhaps instead of charging him perhaps we'll bring this round a different angle and bring him back on line'. It's not about punishing them, it's about maintaining control. \{my emphasis\}

- Male, 4-10 years service, Alpha Jail.

However, only certain officers could use this tactic effectively. Inmates often would not accept informal immediate discipline from a newer officer. Experienced officers would also freeze newer officers out of informal techniques, as they did not 
sufficiently trust them or have confidence in their ability to enforce them. For example, they would prevent the newer officer from accompanying the senior officer and inmates to the pound to witness what may be about to take place. At the same time, however, newer officers were also expected within the officers' informal code not to use formal disciplinary procedures, making control particularly problematic for these officers to achieve. As far as inmates were concerned, an officer needed to have earned the right and ability to 'interpret' the use of informal rules:

\begin{abstract}
I think service and size have a lot to do with it. An officer can be here like about a year without having any bars up and thinks he gets on quite well with the inmates but the reality is he doesn't. He can't get away with what we (senior officers) can. Like I can walk into an inmates cell 'turn the music down' 'get stuffed' - so I switch the power off. He can nut off in there all he likes. Another officer will come down and turn their power off and when he (inmate) gets unlocked he's going to make a beeline straight for him, you know. Its funny really, I can go 'turn that music off' and they go 'and who are you?' and then you hear this whisper all the way down the wing - whew - the wing splits up and I yell down 'I'll smash that fucken radio if I have to come down there' and they know I’ll do it.
\end{abstract}

- Female, 4-10 years service, Alpha Jail.

Overall, the changes in staff group cohesion had led to the rules of informal discipline becoming increasingly unknown yet, at the same time, had encouraged the practice of new modes of informal discipline. As officers were aware that they could no longer rely on the loyalty of other officers not to report them, they were less likely to 'go round the back' and more likely to apply less physical means such as extra strip searches. Thus:

\footnotetext{
... nothing worse than they're listening to their stereo and then there's no power (laugh). There's been no physical violence, yet it would be far more unpleasant... so you can actually piss them off a lot more by doing it that way than by resorting to violence or charges.
}

- Male, 4-10 years service, Alpha Jail.

The rules of engagement also had to be renegotiated to take account of inmates' rights and ability for recourse, leaving uncertainty for both inmates and officers. Uncertainty in the prison could lead to increased violence from inmates and anxiety amongst the officer group: 
You've got some guys who have been through the system since they were this high and that's (physical discipline) the only thing they know. And there's one guy, like he'd get a crack from an officer and he was fine, it was almost like that was an expected thing. But another guy won't be fine, he'll put it on paper. It's hard to know, so you have to be careful now.

- Female, 4-10 years service, Beta Jail.

As it was, the use of physical force to control prisoners did happen, albeit infrequently. To protect themselves after such incidents, officers would reframe such events, justifying them on the lines that the violence was merely self-defence; that they had had to deal with situations spontaneously with whatever tactics seemed required; or that what had happened had been in defence of the group:

If someone’s going to hit you - defend yourself! You don’t have to take nothing. You didn't become a prison officer for that reason and I say 'you hit'. I'm not asking you to go and bash them. But if someone is going to hit you, you defend yourself. I don't care what anyone thinks.

- Male, 10+ years service, Alpha Jail.

Officers reported that their attitudes to violence as a form of informal discipline tended to mellow over time, as Goffman (1961) would suggest - a reflection of their 'moral career development'. What may have seemed barbaric or abhorrent early in their career became more acceptable as they were desensitised or exposed to more situations and the effects of particular behaviours within the environment:

When I came here I was so anti staff assaulting inmates. I'd get phone calls from staff, you know, ringing to talk because they had been made to be watch out. You see staff are people too. Its like 'what do I do about it?' and I say 'well you have to make your own decision, I can't help you there'... but my attitude changed. You see this guy come to work and he'd been assaulted and I said 'what have you done?' He wouldn't say anything to me, he just said 'oh we had a one-on-one' and I said 'oh yeah, well he got the better of you didn't he?'.... Anyway, later that afternoon this officer came to the unit and I said to him, I said 'look, this boy, he's actually had an attitude adjustment, I want to congratulate you' and I said 'what a wonderful difference to the inmate'. He was just a pain in the arse and him having a one-on-one actually, you know. I don't approve of bashings, but I said under the circumstances, it was one-on-one, it was because of an attitude problem, and the end result was a positive one, I don't have a problem with that. \{my emphasis $\}$

- Female, 4-10 years service, Alpha Jail. 
However, such violence was not expected to be arbitrary and ad hoc. On the contrary, there were set 'rules of engagement' for such confrontations which both inmates and officers understood and these had remained constant and reflected the traditional officers' script. These included: not backing down once the challenge had been laid; the fight had to be fair, one-on-one; not kicking a man when he was down; and, regardless of the outcome, there were to be no repercussions:

\begin{abstract}
If you call their bluff you've got to be prepared to go toe-to-toe. Sometimes it's easier to say 'pull your head in'... it just depends what frame of mood you're in yourself. Sometimes you might think 'I've had a guts full' and so you just turn and say 'right let's go to the gym or the pound or whatever', front him up. But you've got to be prepared to go for it and then if you get knocked over then 'tough luck', you've got to wear that, there's no paperwork.
\end{abstract}

- Male, 4-10 years service, Beta Jail.

On the other hand, if an inmate had assaulted an officer, retaliatory violence was frequently applied. This was important to regain balance within the wings, and it could come from officers or inmates:

\begin{abstract}
I haven't actually seen it happen but I've heard about it. It's like one time, [male officer's name] came to work and I don't know what he did but he went down the pound and he went and said 'I heard you assaulted one of my staff, that's not the done thing' and I don't know whether he whacked him or not, he gave him a bit of 'counselling'. It's that group protection thing again, you know.
\end{abstract}

- Female, 4-10 years service, Beta Jail.

Inmates will actually knock over an inmate for knocking over an officer because they (inmates) know the flow on effect on the way the next few weeks or whatever will be. If an officer gets knocked over in a unit then basically everything goes into reaction mode and the whole place, I wouldn't say shuts down, but goes on a go slow until the whole thing settles down and there's a happy medium again.

- Male, 4-10 years service, Beta Jail.

In the past, such retaliation by officers would occur no matter which officer had been assaulted as the maintenance of group solidarity and loyalty was paramount. However, as group solidarity had been weakened and officers could no longer trust all staff to keep quiet about such behaviour, they were not prepared to put themselves in a precarious position for just any officer and were careful when they would participate in such behaviour: 
It depends what sort of officer he (inmate) hits. There are the new officers on the job that we don't like. They create the situations. So because of the change, people tend to be more reluctant to deal with the inmate. But if they hit one of my mates I will go and do whatever needs to be done... you look at it the same way as if somebody tried to hit your daughter, you would go and try to fix that person up, you know. Just to make sure it doesn't happen again. Right? Wrong? There's no such thing - it's what is necessary. \{my emphasis

- Male, 0-4 years service, Beta Jail.

Here then is an illustration of the way in which the previous unity and homogeneity that had marked 'handling your lag' had been replaced by layered divisions. This officer would go to the physical assistance of those who come within his circle of mates, but had become much more guarded about extending such 'collegiality' beyond them.

\section{Writing Ethnicity Into The Scripts}

As a consequence of the affirmative action policies and new recruitment strategies of the early 1990s, ethnicity had come to play a much bigger part in the life of the prison. Initially, as 'brown' officers were introduced into it, Pakeha officers maintained their dominance through rank and numbers with a clear ethnic us-them split occurring within the wider group:

I was getting on with both sides and I was given the option 'you're with us or against us' - and that wasn't from the Maori side believe it or not.... There's been a lot of divided issues because suddenly there was a high Maori staff ratio and only a few of us honkies. It became a dividing issue. There were times here where you'd work an eight hour shift and you wouldn't speak to your offsider (other ethnicity) at all.... there were incidents that happened here and it became a divided jail of them (Maori officers) and us (Pakeha officers). \{my emphasis\}

- Pakeha, 10+ years service, Alpha Jail.

However, by the late 1990s, being white had become an encumbrance whereas being brown had benefits. While still the minority in terms of numbers, Maori and Pacific Island officers had informally asserted their dominance through the influence of their networks, groups and cliques; and formally because of the emphasis on Maori cultural values - especially by the prison authorities. This then addressed the ethnic mix of 
inmates (more than 50\% Maori) and bi-cultural obligations under New Zealand's founding constitutional document, the Treaty of Waitangi. Again though, such developments seemed to downgrade the values and tradition of the essentially white prison officer cultural history and script. The ethnic imbalance in the prison staff and formal removal of rank status also contributed to this realignment of officer dynamics and power. Maori and Pacific Island people could adjust to this quite easily as they were moved from their position of marginality outside the prison to an inside position of dominance. Pakeha, however, and particularly longer serving officers amongst them, were not able to accommodate these changes in dominance as easily.

Their change in status and power created a great deal of resentment on their part towards ethnic officers. In addition, the more experienced officers lost formal status as a result of the removal of the rank structure and demotion of many Acting Unit Managers. These longer serving officers preferred to work within 'Senior Pakeha Units'. Ironically, such congregation exacerbated the position that senior officers found themselves in as they no longer acculturated new officers and yet blamed them for their deviant interpretations of the traditional prison officers' script that they still predominantly followed. By congregating within such units they effectively removed themselves from the influence of the ethnic networks and cliques, and were therefore able to maintain elements of the traditional script and continued to work with the old style of 'by the book'. However, this old style 'by the book' was quite different to the new style 'by the book' that less experienced Pakeha officers tended to abide by. Within the old style, officers maintained the interpretation of formal rules that were supplied by PIGO and in many cases this was compatible with the new working practices. Such officers seemed particularly rigid and inflexible to change or irregular routine. As an example, if a set of keys were placed back in the key press on the hook only adjacent to where they were supposed to be, the old style officer would become distressed over the situation for several hours whereas for the new style officer ‘close enough was near enough'. The new style 'by the book' was a more perfunctory approach to work that permitted more flexibility than the old style and was thus more likely to reject those aspects of the traditional prison officers' culture which required involvement in the prison beyond the 'perfunctory': for example, the use of violence within conflict situations or association with officers outside of work hours. At the same time, however, as we have seen, the newer officers were frozen out of learning 
how to negotiate control effectively or how to use alternate informal means of control. Unable to master this aspect of the job, they were viewed by both experienced Pakeha and ethnic officers as ineffective or weak.

The only Pakeha officers who were accepted by ethnic groups were those with a military background. Ex-military personnel were more easily able to achieve this, because they were familiar with closely bonded group behaviour from their service experience. Overall, though, Pakeha officers were working in a marginalised occupation and at the same time their place within the officer body and its values had also become precarious. Pakeha officers of less than six years service, that is those who entered the service after the influx of ethnic officers, experienced acute problems of acculturation as they were generally unwilling to accept the dimensions of the new prison officers' script that Head Office had written for them, with its strange injection of ethnic values and practices, but at the same time they were unable to challenge its existence. This became even more problematic as they were not mentored adequately due to the lack of experienced officers. They were thus less able to adjust to the understood ways of being nor able to buttress the pains of their occupation within the prison. On top of this, they then found they needed to prove themselves more than non-Pakeha to be accepted and trusted by the officer group:

\begin{abstract}
In the beginning it is harder as a white officer. As time goes by it's just the same, as soon as you've proved yourself. It's just they (inmates and officers) consider us upper class or whatever. A lot (of non-Pakeha officers and inmates) have had bad experiences with white people on the outside so they make us work harder to prove ourselves in here.
\end{abstract}

- Pakeha, 0-4 years service, Beta Jail.

In contrast, the ease with which Maori officers were accepted and the difficulty experienced by Pakeha officers caused resentment amongst the Pakeha staff at what they saw as the elitist attitudes of Maori officers (contra Jacobs \& Kraft, 1978; Owen, 1988):

They say that they'll make a Maori unit, so that it's just a unit run as a marae ${ }^{151}$, one for Maoris $\left(\mathrm{sic}^{152}\right)$. To me that's absolutely ridiculous because to me it's

\footnotetext{
${ }^{151}$ The term marae is the Maori word for the central area of a village and its buildings; a meeting area or focal point of a settlement (Ryan, 2001).
} 
making an elitist situation - and we've already got that here! You've had things like kapa boogie ${ }^{153}$ and things running in here and the inmates that go to it become elitist which can have adverse effects. Its segregation within prisons and it's not just the inmates. You see the Maori officers and they're all elitist and all that - its apartheid!

- Pakeha, 4-10 years service, Beta Jail

It was also felt that some Maori staff took exception to having Pakeha in positions of power within the officer ranks, such as the Unit IC role, something that added to Pakeha frustrations:

It's (racism) something I don't want to get involved in. I was (a) victim of it in [Beta Jail Unit] in a reverse way. It was quite serious. It was with the (unit) manager down there. It's an extremely upsetting thing to have happen. It's a real political hot potato. You mention racism - no one wants to tackle it! They don't want to hear the word... there was one person (Maori officer in the unit) who didn't want a Pakeha as his IC and I was the IC of the unit (speaking officer was subsequently stood down)... now at the end of the day the bosses said they know it was badly handled but that's history and we've got to get on with it.

- Pakeha, 4-10 years service, Alpha Jail.

This extract highlights the sense of powerlessness that Pakeha officers experienced in the face of the new ethnic power relations. Pakeha felt they simply had to 'get on with it' or 'handle it'. Paradoxically, given the way in which Pakeha had become the minority group, in terms of values if still not in numbers, accusations of racism against Pakeha were also frequent. For example, it was not uncommon to receive such comments from other staff when a Pakeha officer had problems with non-Pakeha inmates:

You know that [inmate's name] threatened to stab me, he was Samoan, and I was asked by my Unit Manager if I had a problem with Samoans! You know, and I sort of said 'I'm surprised you're asking?' But that (the threat) wasn't for any reason, anybody could have been locking him down, it just happened to be me.

- Pakeha, 0-4 years service, Beta Jail.

\footnotetext{
${ }^{152}$ Plural of Maori is simply Maori (Ryan, 2001).

${ }^{153}$ Non-Maori officers often used the term kapa boogie in a derogatory manner to refer to kapa haka (Maori cultural dance).
} 
Pakeha were aware that government recruiting policy favoured Maori and Pacific Island people rather than themselves and although they could see the logic in this decision, understanding the rationale did not make their pains any easier to bear:

\begin{abstract}
We get a lot of Maori and Pacific Island inmates. These guys, you know, the cultures, they need to be able to link to their own. Like we talk to them and it can be quite confusing, you know. They don't quite understand or whatever. But if they've got Pacific Island and Maori officers and that, you know, it's just a different level and they're able to relate and talk to them.
\end{abstract}

- Pakeha, 0-4 years service, Alpha Jail.

Each ethnic category, however, had their own pains associated with the emerging new style of prison officer work that was now expected. In contrast to the problems of Pakeha officers, Pacific Island officers felt particularly aggrieved at having to deal with all 'Island' inmate problems. Kanter (1977) described minority groups with less than $15 \%$ within a large corporation as token employees. Pacific Island prison officers fell into this category. These token workers tended to have high degrees of visibility and performance pressure, and tended to be placed within stereotypical roles and assignments creating role encapsulation for this group (see also Jurik, 1988). Already under pressure because of their thin distribution across the prison, this placed a good deal of additional stress on their workday:

I actually feel it a lot. It's like anything that happens to an Island inmate they come to us straight away. Even if the inmate speaks no Samoan or Tongan or Fijian at all, all he speaks is fluent English? They seem to come to us straight away, because they, I don't know, it's passing the buck or, being culturally sensitive or whatever. But from now on I'll say to them 'look you deal with it, he speaks English, you sus it out'. I do tend to carry on board more responsibilities than others. It's different for Maori (officers) (laugh) most of them don't even speak Maori!

- Pacific Island, 4-10 years service, Beta Jail.

A Pacific Island officer commented ${ }^{154}$ that he felt 'used' by other officers and management for his language and ethnic knowledge. While he enjoyed the benefits of easy acceptance and instant status, the negatives outweighed these positives. He felt Pacific Island officers were 'dragged' anywhere around the prison to act as translators

\footnotetext{
${ }^{154}$ During the interview with this officer he requested the cassette recording be turned off while he made a few additional comments. He said he did not mind me using the information but did not want it recorded verbatim.
} 
and never received any formal acknowledgment for their efforts. In particular, he was angry that Pacific Island officers were frequently called into potentially volatile incidents because they were all 'big, tough and able to fight' - the very characteristics that had made their acculturation to the prison and acceptance by fellow officers easier. He agreed that most Pacific Island males could 'handle' themselves in physical confrontations, but thought it unfair that they were always called into the dangerous situations as 'nobody wants to get the bash on the job'. He also reported that he had repeatedly attempted to transfer from his particular unit but the Unit Manager expressly told him to stop, as he would never sign the transfer form. This officer felt that there were advantages to being brown but more so for Maori officers because all they needed to do was 'cry racism' and everybody 'jumped through hoops' to keep them happy. However, if a Pacific Island person used the same line they were dismissed, stereotypically, as a 'stupid Islander'. As another Pacific Island officer asserted, the system was not designed for them:

I looked at it (the prison service) and thought 'my God', you know, 'what am I doing, this is basically a Maori and a Pakeha company', you know. 'How am I going to bridge the gap?' I mean for years here there are so few Pacific Islanders in these jails.

- Pacific Island, 4-10 years service, Alpha Jail.

As for Maori, even though they had become the dominant group in the prison officer set, some felt stereotyped and 'trapped' into certain roles by their ethnicity. While they experienced fewer pains of acculturation, they effectively did not have a choice regarding whether or not they were going to accept the ethnic dimensions of the new Head Office script. All non-Maori officers simply assumed that they would become involved with the ethnic groups or cliques. It was clear from discussions with the Maori officers that a number would have preferred to take a more perfunctory approach to the job and their colleagues, but there was significant peer pressure, especially from those ethnic officers who did belong to the cliques. If a Maori officer appeared to question the dominance of their ethnic values in the life of the prison, they were rejected by their peers. At the same time, Pakeha also rejected them as it was assumed they were 'in’ with the cliques. 
Overall, the injection of ethnicity into the officers' scripts had had a profound effect on their occupational culture and working dynamics. Ethnic culture came to dominate creating new pains of occupation for Pakeha officers who were powerless to challenge this aspect of their work. Many senior Pakeha officers retreated to 'Senior Pakeha Units' which, while insulating them in part, effectively compounded their problem as they were no longer involved with the transmission of the traditional officers' script. Newer style Pakeha officers attempted to insulate themselves from their pains by retreating from the culture, instead taking a perfunctory approach to their work and the group. Paradoxically, this actually contributed to their pains of occupation as they were then unable to use the aspects of the culture which buttressed other pains. In contrast, ethnic minority officers, although experiencing their own pains of occupation, found themselves in positions of power and dominance that were denied them outside the prison.

\section{Writing Women Into The Scripts}

Prior to the introduction of women as officers, the officer culture was dominated by masculinity, machismo and perceptions of physical prowess. Prison was viewed as dangerous work, one of the few status enhancing features of this dirty job (see Crouch, 1985). The introduction of women as officers challenged this perception. Women experienced considerable opposition from male officers when they were first employed in male penal institutions (see Fry \& Glaser, 1987; Ministerial Committee 1989). During my research, many male officers remained resentful that women had 'intruded on their patch':

Let's get this straight madam, who are the macho people of New Zealand - the male rugby players, right? Who do we employ - male rugby players. Most New Zealand males play rugby and they like to be macho and tough.... The macho male will be the ones who do not like the female intruding on their patch. Got that?

- Male, 10+ years service, Beta Jail.

Even the tone of this extract demonstrates this senior officer's disdain and patronising attitude towards women, any woman (including myself), intruding on his patch. 
Over a decade after they had been allowed to work in male prisons in New Zealand, women continued to work in a generally hostile environment where opposition, discrimination and harassment were faced daily. While the barriers within other male dominated work forces may have been decreasing, the barriers within Corrections remained strong and greatly effected women's daily experience (see also Zimmer, 1989). It was not the formal structures which affected women-there were, after all, affirmative action employment policies_but rather the informal mechanisms of dominance and control by the officer group. Longer serving women recounted the hostility they experienced:

\begin{abstract}
I look at a lot of the females here and I think 'you have no idea the road I've come to get you people in this place'. They really don't. The women that were here before me, as I understand it, were here for no more than about two years a piece, and the way they were treated wasn't kosher and that's why they resigned. The men, of course their version of events is 'but we supported them, they weren't up to the grade'. But having been down the track myself, when I started, they didn't want women here, oh the tricks they (male officers) used to play. We didn't fit the stereotypical image of the prison officer. So we not only had to deal with the prejudices of the inmates, we also had to deal with the prejudices of the male staff also. And the support wasn't there at all. I learned to become an independent and upright person to survive. I had to survive on my own merit because there were many situations where you would turn around and you would be, you know, by yourself (without support in the wing).
\end{abstract}

- Female, 4-10 years service, Alpha Jail.

Some male officers argued women would not be needed if the Prison Service were not employing these new 'weak' males:

\begin{abstract}
It all depends what officers are on the floor, strong officers, you know, reliable officers, who whatever they say goes, then you don't need any women officers because that's how it is, you know. But if you look at our unit, you have officers that are weak, who won't go all the way, you know what I mean. They're not courageous to face the situation. So then you need women because it's better for women to calm the inmates down. For example if I've got to go and calm them down, I lose my temper - you know what happens. But if you have a female officer the inmate calms down.
\end{abstract}

- Male, 4-10 years service, Beta Jail.

The notion that women compensated for weak males was not linked with any inherent qualities of the women that surpassed the newer males. Rather, it reflected the view that women, at least overtly, were more adept at playing along with whichever script was dominant at the given location or time. Women neither had the power of numbers 
nor status to challenge the values and expectations of whichever male officers or groups of them they were dealing with so they generally fell in line with them:

It's silly really, one day we do it this way and then the next day it's some other way cos we got some other IC and that. I really don't care as long as the work gets done and everybody's happy. Yeah, whatever, you know.

- Female, 4-10 years service, Alpha Jail.

However, male officers were beginning to recognise women officers' ability to have a calming effect on the inmates, which decreased the number of incidents and which could also decrease the seriousness of an incident if a woman officer responded to it:

It's a guy thing, you know, you're in people's faces over bugger all. It's like anything 'you bastard' and automatically you've got somebody standing there and eye-balling you, what do you do? But you see women aren't like that, and that was one of the influences of women. God dare I say it, they were able to bring a calmer solution to some of the issues. And I think that was a good thing because women could defuse some of the situations whereas the men would be snarling and snapping, you know what I mean.

- Male, 10+ years service, Alpha Jail.

It does take a lot of pressure off the males (officers), and I don't think the males really understand that. There are some real macho staunch guys (officers) out there, that's fine, but at the end of the day they have to come back to women and say 'can you go deal with that situation' because they don't know how to relate to that situation. It's good for the jails and the men (officers and inmates).

- Female, 4-10 years service, Alpha Jail.

These two extracts demonstrate a gendered difference of the perception of women as officers. While the man directs attention to the 'calming' influence associated with confrontations, the woman focuses on the 'caring' role that women fill. The latter also indicates the marginalised and 'invisible' nature of women's experience. For many, the dominant belief was that male officers did not value their contribution - yet, at the same time, were quick to highlight any discernible 'inadequacies'.

Notwithstanding this, male officers did recognise other positive effects of women as officers. For example, some asserted that female colleagues brought a civilising influence to the conduct of both the inmates and male officers: 
This inmate, a couple of months after I'd been in the job, he says to me 'you know it's nice having females here', he says 'I notice that the swearing had gone down, the male officers tidied themselves up a bit'. Inmates would still swear but sort of 'sorry I didn't mean to say that'. It was funny cos the inmates tidied themselves up too eh, started having proper showers and washing their cloths (laugh).

- Female, 4-10 years service, Beta Jail.

If, however, women were employed to 'normalise' the prison environment, it could be argued their role was ‘prison officer and woman' whereas men were simply 'prison officers’ (see Morris \& Hawkins, 1970; Myers, 1995; Peterson, 1982; Wicks, 1980). This placed additional pressures on this already marginalised group.

Overall, the presence of women officers still represented a significant challenge to the existing prison officer culture and power relations. Notwithstanding recent research which suggests that the emphasis on masculinity and physical strength has been eroded in prison (see Crawley, 2004; Liebling \& Price, 2001), I would argue that the change in culture, in New Zealand at least, had merely produced an altered presentation of 'masculinity'. It seemed women were perceived as 'fair game' if they dared challenge the masculinity of the prison by joining the service. As such, female officers had to contend with extensive and continuous sexual harassment from male officers, management and inmates. The responses of female officers ranged from endurance to intolerance:

There was this other lady, [female officer's name] when I first started who was shocked that anyone would call her a 'screwbitch' or anything like that, and I always thought if you were that worried about your sensitivities why don't you have a different career choice because you've got to expect comments.... Let's be honest, the fact that you've got boobs just opened yourself up. So I don't think you can be thin skinned... if you've got too many sensitivities get out.

- Female, 0-4 years service, Alpha Jail.

I can only take so much of the sexual comments, and in the end I get annoyed with it and I just tell them to keep the comments to themselves. Whereas a lot of the other women will turn around and walk away which is the wrong thing to do. I've told a few of the women that, you know, 'if you don't like it tell them and if they start giving you lip come and see me and I'll go and see them'. But they have to learn to be able to say to the guys 'just cut if out, I've had enough of it'. Sexual harassment in this place is, I think it's quite big for women, but they will only do it to women who won't say anything, you've got to fight back.

- Female, 4-10 years service, Alpha Jail. 
Women officers also had to be constantly wary and protective of their sexual safety, while men obviously did not have to endure such pains of occupation:

\begin{abstract}
Sexual harassment and all that, that's nothing. Men just don't give a squat. I know, I was sexually violated by an officer. I mean there's just been so much stuff like that. I kick myself right now, I should have done what I was gonna do then and just lay charges. But I got threatened by a mate of his who was 'you do that girl and you won't have a job, your life will be so miserable and you won't know what hit you'.... They close ranks completely, they just close shop, that's the way it is, and I'm not the only one.
\end{abstract}

- Female.

While women could complain to management about sexual harassment, they were shown no sympathy and given no assistance. The message was the same as for the stressed out male officer - 'handle your lag' or leave (see p. 207). Women thus had to find their own way to deal with difficult situations:

\begin{abstract}
I was in the guardroom and the unit manager was out there (in the wing) and came up (to the guardroom) and I was screaming at him 'you don't know what it's like to come into work and being told that they masturbate over you. You don't have them standing in the doorway looking at you, telling you how they got a hard-on over you last night. You don't have to deal with any of that, they'll be lying on their bed naked and masturbating knowing that you're going to be looking in that window' (doing a muster check). I don't know, they (management) weren't interested. It was like 'handle it'. \{my emphasis\}

- Female, 4-10 years service, Alpha Jail.
\end{abstract}

As this extract indicates, the lack of support from management, or even from other officers, could result in emotional public outbursts - reinforcing the male officers' generalised perception that they were indeed not suitable for the job. For many women, though, the experience was that they 'suffered in silence' as not even their fellow women officers could support them given their division and atomisation.

But at the same time, if they did 'handle their lag', they were categorised into stereotypical 'ideal type' roles: mother, pet, promiscuous or staunch bitches ${ }^{155}$. For

\footnotetext{
155 The New Zealand context closely mirrored Jurik (1988, p. 292) who viewed the pet as incompetent, innocent, weak and in need of male protection. The seductress ('promiscuous' in the New Zealand context) was sexually desirable and potentially available. The mother was supportive and scolding. The iron maiden ('staunch bitch' in the New Zealand context) was competent in her work but harsh, cold and asexual. These were ideal types, hence women were not static and frequently reflected components of multiple categories.
} 
example, any woman who received promotion or preferred task secondment was thought to have done so through sexual favours. They were then seen as 'promiscuous', which created sexual tension and competition amongst the male officer group. Worse still, if women officers became sexually involved (or were thought to be) with inmates it breached the officer-inmate relationship rules and was a challenge to security and control:

\begin{abstract}
Women! Well I'm probably not the best person to talk to because I've been in units where I know four of the female staff have become involved with inmates... that's four in the last eight years, which is quite high really. What it does is it just jeopardises the whole security of the institution and you have to be careful what you tell her cos, you know. And if you suspect something is going on, what can you say until you know something is going on. And then it just all turns to custard. Inmates don't like it either for obvious reasons, jealousy issues, you know, that a particular inmate's getting special attention.
\end{abstract}

- Male, 4-10 years service, Beta Jail.

Indeed, women were assumed 'guilty until proven otherwise', whereas the opposite applied to men suspected of indiscretions, and excluded, particularly from information (an important aspect of their occupational culture), on this basis. As such, women officers felt that they had to prove themselves more than male officers (see also Owen, 1988) and received only tokenistic credit for their efforts:

I think in a way you have to prove yourself more to the guys, you know. It's a respect thing and a trust thing. It's like 'she can handle it, that's cool'. You get a reputation with that, yeah, where a guy could probably plod through quite easily.

- Female, 0-4 years service, Alpha Jail.

The problem for women was that the testing and requirement to prove themselves as capable officers continued relentlessly even after they had passed initial character testing strategies, which all new officers went though (see chapter five):

You get guys who start on probation who feel they don't have to listen to you because you're a female. And you find yourself going 'you probationist, me two bar, you do as told' 'why should I do that?' 'Because I've been in this job and I know what I'm talking about even though I'm a woman'. I've been there and done that, you know. You feel that you're constantly trying to prove yourself and you're thinking 'why do I have to do this every bloody day? Why am I trying to prove something to somebody? Why should I have to, blokes don't!'

- Female, 4-10 years service, Alpha Jail. 
Regardless of which stereotypical category the woman was, they were viewed as a weak link within the officer group. 'Mothers', for example, were accused of being 'played for a fool' by inmates:

\begin{abstract}
Men tend to think that you're an easy target. I've seen it happen to so many women's lives as prison officers. It's 'you're an easy target' or it's 'ah you're a sucker'. You come along and they (inmates) can tell you anything and you know, you'd fall over yourself trying to help them and all. But you learn these skills of differentiating, I tell you, there are some master manipulators in here.
\end{abstract}

- Female, 4-10 years service, Alpha Jail.

With regard to 'pets', male officers were concerned about their supposed lack of physical prowess:

\begin{abstract}
I think we have to be very careful about the suitability of women. Women tend to be smaller, petite. You sometimes doubt their ability which is probably not a genuine concern to have because we don't have day-to-day situations in this institution where there is a necessity to be physically able to quell up an aggressive and violent male. I'm still pretty ambivalent on the thing about women.
\end{abstract}

-Male, 4-10 years service, Alpha Jail.

These stereotypical categorisations were also influenced by the ethnicity of the women officer (contra Walters, 1993). Pacific Island and Maori women tended to fit the role of mother or staunch bitch, while Pakeha women were typically cast as pets or promiscuous. Pakeha women officers had additional issues to contend with. Within the dominant New Zealand ethnic gang, the Mongrel Mob, women were below their dogs on the hierarchy - Pakeha women were even lower. Gaining compliance from the members of this gang when they came to prison was impossible. Having no legitimate authority with this prominent group led other inmate groups to question the position of these women. Pakeha women officers found working with Pacific Island inmates problematic for similar reasons:

It's like with the ladies, you find (that for) a lot of the Island boys women don't rate very highly in their pecking order. So when you've got male staff on, the guys (Pacific Island inmates) are good. But sometimes when the ladies are on they tend to fly off a bit. Not with the Samoan ladies though.... It's just the way they are. It's just in their culture.

- Pakeha Male, 4-10 years service, Beta Jail. 
Overall, then, women had been less successful at securing a position within the dynamics of the prison. Although they had been written into the new script by the prison authorities, many male officers continued to disregard this. Women were, at best, treated as token males if they proved themselves or placed them within one of the 'stereotyped' roles. At the same time they were subjected to continued harassment and indignities and had to be protective of their sexual safety. In this way, being a woman had problems of acceptance within the culture, but being a Pakeha woman was a double disadvantage because neither the officer nor inmate groups recognised or respected them. Theirs was an exceptionally difficult lag. For male officers, while they conceded a few nominal benefits of women as officers for day-to-day control, senior officers continued to assert that they would not be necessary if the prison authorities were to exclude the new style weak officers that they had similarly written into the script.

\section{Gossip, Knowledge, Power}

Despite the changes taking place in the prison performance, there was one aspect of the job that remained of vital importance - the ability to gossip. By indulging in this, any officer could elevate their status in the group hierarchy. Notwithstanding the emphasis on difference and excitement in the new Head Office script, as we have seen what was still highly valued by prison officers was certainty and consistency. What was needed to safeguard these qualities was knowledge. This therefore meant knowledge itself was a valued and transferable commodity. Everyone was viewed suspiciously and at the same time as a potential source of information. Information gathering became an important activity amongst the officer group. Indeed, the more random the piece of information, the more exciting it was to them. Every communication encounter between officers, between inmates and officers, between management and officers, between ancillary staff and officers, even between outsiders and officers ${ }^{156}$ developed into an information game. Even when these took the form of affable, everyday encounters, they were linked to assertions of power attained via the

\footnotetext{
${ }^{156}$ An example of such an exchange, as mentioned in chapter two (see p. 102), was when a group of officers decided the way to ascertain my true motives was to get me drunk and then fire questions at me 'surreptiously'.
} 
acquisition and subsequent transference of gossip into knowledge. As an example, while an officer and I were sitting in the gym one day watching a group of inmates exercising, a bored inmate came over and sat with us. Pleasantries were exchanged and a non-specific rather uninteresting conversation developed. At the end of the exercise period the inmates filed out and the officer whispered to me excitedly 'did you hear that... his Mrs is pregnant... I bet I'm the only one that knows that - now who am I going to tell first?’

Officers took information gathering to such an extreme that a status hierarchy had developed, whereby officers who were more 'in the know' were more respected. To realise one's potential within the prison system, officers needed to learn quickly that the most important part of the day-to-day job was not linked to any of the formal objectives such as safe and humane containment but instead was predicated on inserting oneself into the information status hierarchy. The 'prison trivia savant' became a very important person, and a very desirable status for officers to aspire to:

\begin{abstract}
Every unit has its go-to person if you want to get things done round here. Like [male officer's name] he's the man when it comes to one-on-ones (laugh). Guess it helps being built like a brick shit-house! [Another male officer's name] has the smoothest shifts (as Unit IC). I know it's going to be a good shift when he's at the controls. Then there's [female officer's name], you want to know anything you go to her, she's like a walking prison encyclopaedia. If she doesn't know it it hasn't been thought of yet (laugh).... We call her 'the shadow' because she's always lurking around corners listening to shit. \{my emphasis\}

- Male, 0-4 years service, Alpha Jail.
\end{abstract}

There was, though, a difference in information seeking requirements between Alpha and Beta Jails. Officers who transferred jails quickly redeveloped their techniques to match the jail that they were in. Within Alpha Jail, prison trivia was based around 'knowing inmates'. Within Beta Jail it was based around 'knowing officers'. For Alpha Jail officers the priority was security both of the jail and the officer group, and for Beta Jail officers the priority was officer group solidarity. In this way, the purveying of gossip was tailored to fit the operational routines of the particular jail, as did its architectural structure. Crawley (2004) noted the importance of a back stage for cathartic displays of emotion. Here, the physical differences between Alpha and Beta Jails similarly influenced officers in their information seeking behaviour. Beta Jail afforded significant back stage space where officers could quietly converse with one 
another or with inmates ${ }^{157}$. For example, the staffroom was out of view of inmates. Similarly, on the pretext of performing a security check, officers could 'disappear round the back' of the unit, out of audible range and sight of all other actors. Alpha Jail, however, had no such area. The guardroom was the only reprieve during shift time, but was in full view of inmates and was frequently interrupted by them. Beta Jail officers, therefore, could easily and regularly withdraw from the front stage, whereas Alpha Jail officers had limited non-public space, other than the staff facility during breaks (which frequently did not coincide with other like-situated officers) or during night watch, in which they could share personal details at a more meaningful level with other colleagues.

Alpha Jail officers argued that they needed to know every possible detail about the inmates in order to monitor behaviour and any changes that might lead to trouble:

\begin{abstract}
Pay attention to any detail that the inmates are doing. Like certain inmates that could be playing ping-pong, and all of a sudden they're sitting in their cell, you know, 'why are they doing that?' 'Why is he going into so and so's cell when he never goes into that cell?' Those things the book misses out on. The book doesn't tell you that but it's so important for knowing the mood in the wing, any little change could mean something's brewing. \{my emphasis\}
\end{abstract}

- Female, 4-10 years service, Alpha Jail.

The level of information sought included: inmates' links beyond the prison (family, friends, associates, enemies and so on); potential conflicts with visitors (police informants, ex-partners, rival gang associates and so on; see also Wacquant, 2002); potential links with other inmates (criminal associates/ rivals, relatives and so on); prior and current offences including those for which they had not been convicted; and minute details of their daily personal routines (how long they typically spent in the shower, whether they ate fast or slow, who they associated with and when and so on). Essentially, anything and everything was important, no matter how trivial. This was because in the eyes of the prison officers, such tiny scraps of information had the potential to safeguard prison security:

\footnotetext{
${ }^{157}$ To compare Alpha Jail and Beta Jail back stage space see Appendix E’s schematic images of Alpha and Beta Jails and note the small guardroom areas of Alpha Jail relative to the large guardroom and private staffroom of a Beta Jail unit.
} 
Staff choose to know and find out because a lot of their offending and stuff might have caused a series of events, might have follow on repercussions on the inside. I might be looking at the newspaper or the warrant and you can say 'he assaulted her', and my sister lives next door so you ask her for details. It doesn't take long to figure out where an inmate comes from and everything about them if you do so choose. You want to know where each other's coming from so you want to learn about each other as much as possible. Could be that the inmate knocked over some other inmate's Mrs., you need to know these things. New Zealand is such a small place and now with them all coming from one region there's bound to be problems.

- Male, 4-10 years service, Alpha Jail.

Officers only needed the occurrence of an occasional incident to justify the need for such vigilant information gathering. These then became prominent in the institutional memory that the officers were able to draw on:

\begin{abstract}
These new guys coming in just do what they want to do and there are consequences of not doing the job properly. Not getting the basics right like seeking out all the information they can and passing it on. Like what I'm going through at the moment (prolonged court case concerning the death of an inmate while in custody), that's a classic case of staff not passing on information. They didn't pass on what they'd seen and the follow on from that is a guy's dead!
\end{abstract}

- Male, 4-10 years service, Alpha Jail.

Alpha Jail officers were also concerned about knowing their fellow staff members, as their circumstances may affect the functioning of the unit:

It comes down to working as a team, and you need to get on and know each other really well. Sort of a trust factor if you really get down to it. You know each other, you know. Prison officers' life has stress on families, their home life and that. And you need to be aware of these situations (personal issues). So (you) need to know so you can trust them, you know. So you know 'oh he's not so good' so you've got to look after each other. It builds that team environment. And you've got to be able to laugh together and release whatever is happening in life, or what's happening at work. \{my emphasis\}

- Female, 0-4 years service, Alpha Jail.

In contrast, in Beta Jail the focus of information gathering around officer trivia seemed to be mainly a way of passing time, trying, rather bathetically, to ensure that every day was indeed different, given that this jail had higher proportions of new style officers who were attracted to this supposed feature of the job, when for all intents and purposes, each one of them knew that every day was likely to be the same as any 
other. The officers' informal code of certainty and consistency demanded that this be so. Thus:

\begin{abstract}
It's easy to pick stuff up because you've got a lot of time to chat with each other. Even though you might be working, there might be two of you in the compound, so you might be standing discussing something together. And if you get a bit of down time, well you can't always talk about prison so you'll talk about something else like 'what did you get up to in the weekend?' 'How did you get on with your hunting?' 'How are the kids?' You pick up stuff like that.
\end{abstract}

- Male, 4-10 years service, Beta Jail.

Information gathering provided a plausible excuse for officer movement through Beta Jail units to ‘catch up’ with officers, as, unlike Alpha Jail, associating with members of other units had to be a deliberate venture given Beta Jail units were physically separated. There were numerous shifts when an officer would travel to every unit, speaking with various staff members and gathering tiny pieces of information of a story, so that they could report back a fuller version to their unit staff. Frequently this was a two-way process whereby they would have to stop at previously visited units to transfer any additional parts of the story. In this way, they were not only purveying a valued commodity—information—but were bolstering solidarity by sharing this with other officers:

\begin{abstract}
I think gossip is important. I think it's a good communication link between staff who don't work together all the time. And to familiarise ourselves also with those staff members that we used to work with ages ago, to get that bond back. You catch up, go over to the unit and have a coffee. Or maybe just a few drinks (alcohol) every month. And it's good because we act the clown, intimidate (sic; derogate) staff, intimidate (sic; derogate) bosses, catch up on the gossip. And I mean the gossip could have been, you know 'this inmate done this' or 'this officer charged this inmate and so on' 'oh but that's not what we heard', you know, those little sort of things. And then you come back on deck and someone will make a comment about 'have you heard about so and so and so and so' 'oh no, that's not what happened, this is what happened' and they say 'oh where did you get that from' 'oh this came from the horse's mouth', you know. So I think it's actually a very important communication link for the staff.
\end{abstract}

- Female, 4-10 years service, Beta Jail.

While the majority of gossip was kept at the social group, clique, unit or jail levels, some pervaded the whole prison and was used to manipulate power relations, particularly in relation to women officers (see also Zimmer, 1989). When I first entered the prison I was pulled aside by several officers, male and female, and warned 
against associating too closely with certain members of the officer body and what would occur if I were to challenge them in any way. Another officer recalled the example that was provided to me at that time during the interviews:

\begin{abstract}
You have to be careful, you tend to find it's those that have been around the system a while and (are) very arrogant and full of themselves. In the case of [female officer's name], she would socialise and go to parties. They took advantage of that and set her up. And they did what they did (gang rape by officers) because they felt it was their right. That's just arrogance, you know [male officer's name] he is that arrogant old school 'oh don't I just love women and don't they just love me' and if you gave him an inch he'd be in your bed as quick as. Cos that's the type of male machismo thing going on. They treat you like shit as well because they're frightened that you'll go back to their wives. So they paint the picture to cover themselves 'there was no way (that happened), no sexual violations, they've never taken place, she's just a slut, she offered it'. That's what they do, they paint these pictures because it protects them, they're innocent. You can't hurt them and the truth will never get back to their homes, but we know.
\end{abstract}

- Female.

In this way, the power of rumour and gossip not only separated the genders but also divided the women officers. Rumour not only acted as a form of social control, but also as a form of social division (see also Owen, 1988), particularly in relation to ethnicity. Non-Pakeha women were readily accepted within the officer group as 'sisters'. Pakeha women, however, represented a challenge to the officer group as distinct 'others', unable to be assimilated, and therefore it was these women who were particularly susceptible to having their characters defamed through rumour and gossip. It was also this group which was most vulnerable to sexual assault. In effect, the women's group had been so successfully divided by the culture of the prison that women officers had turned on each other, increasing their pains of occupation rather than providing support.

Overall, then, the daily complexities of the job were becoming increasingly problematic and unknown. There was a sense of knowing within the officer group that the prison culture was 'larger' than any individual, and that it was easier to accept it than try to alter it:

It's something that was here before me and it will be here long after me. There's nothing ever going to change it. There's always an underlying prison culture and I think I've come to accept it over time, you know. Whereas in the beginning if I'd seen somebody give the bash (assault an inmate) I probably would have been 
concerned, made sure it was put on paper and inform my IC and all that. But, I mean, if something like that happens it's just a story (now). I don't know when it was that I sort of started accepting it? I find that the officers that are a bit more unorthodox tend to get more done or achieve more and tend to be more respected amongst the inmates - which is half the goal to getting the job done.

- Male, 0-4 years service, Beta Jail.

However, as Crawley (2004) suggests, and as has been demonstrated in this thesis, occupational cultures are subject to change and evolve over time. The shared understandings that had previously enabled prison officers to handle their lag were unravelling due to the restructuring that had taken place and the multiplicity of scripts that they were now expected to know. Previously, prison officer work had been simple, transparent, with clear relational boundary lines. However, the structural and demographic changes of the early 1990s had transformed this. It seemed that very little had been left unchanged - even the central characters of the prison performance — white, long serving officers — had been pushed to one side in favour of a more ethnically pronounced prison officer group. The whole officer body had become fragmented and divided, leading to inconsistency, unpredictability and an increasingly difficult lag. On top of this, their relationship with management had become more distant and strained and the constantly shifting line with inmates had deleteriously affected their ability to manage and control them, increasing stress and uncertainty within the officer group. All the while, the traditional officer group was supposed to accommodate the injection of ethnic and women officers. With continued resistance from male officers, women were unable to effectively write themselves into the informal scripts. Ethnic officers, however, had begun to usurp much of the power of the traditional officer body, leading to new tensions and conflicts in the prison. 


\section{CHAPTER SEVEN: UNIT DIVISIONS}

The prison authorities had indeed successfully weakened the traditional prison officer culture and group cohesion with the policy and demographic changes of the early 1990s. As well as dramatically changing the working routines of prison officers, it was intended that the establishment of unit management would solidify this achievement and prevent any coalescence of the old style unity. Prison officers and their sense of 'group' would be confined to their unit, thereby removing the ability to think or behave according to one unified occupational culture. This, it was purported, would remove the 'problems' of the past. However, as this chapter will illustrate, unit management led to further divisions and antagonisms within the prison officer group. This was, in part, compounded by officers electing transfer to particular units and residing unit officers 'recruiting' potential members from throughout the prison which led to distinct demographic groupings with varied scripts. In this respect, unit management did prevent any re-emergence of the old style culture. But at the same time, no new, dynamic, more professional ésprit de corps emerged. Instead, unpredictable and unforeseen alliances and behaviours were its product (see p. 34; also Baker, 2007). Dividing the prison into units led to still more divided and independent groups of officers, to the point where prison rules and authority were being seriously challenged and undermined by disparate individual unit cultures and the development of cliques. The prison officer body was too weak, as a result of the structural changes that had been imposed on it, to prevent these fragmentations and distortions.

\section{Staffing The Units}

Crawley (2004) noted that the comparable 'group working' as a result of 'Fresh Start' had increased solidarity at the wing level and that this could weaken the solidarity of the wider officer group when wing staff prioritised their interests over others. In this 
way, New Zealand was not unique in experiencing this fragmentation and competition within the working arrangements of the modern prison. What was different in the New Zealand context, however, was the manner in which officers had been able to manipulate these new arrangements in such a way as to reconstruct the intended unit divisions thereby producing unintended consequences for the prison authority's goals. The introduction of unit management had been intended to split up the original stage on which the prison officers had worked together into small scale insular units. However, over time, these work teams developed into specific demographic groupings and association sets as officers elected to transfer within the various unit locations. Requests to transfer were based more on the affinity between officers within each unit than on the nature of the job or type of inmates within any given unit. Officers maintained a strong loyalty towards their jail type, but developed even firmer allegiances towards their unit - which only intensified as units competed against each other for scarce resources.

The demographic differences that were emerging between the two jail sets, as discussed in chapter four, were more pronounced at the unit level. The exceptions were in relation to gender and education level distribution. Although there were more women within Beta Jail, generally women were spread evenly throughout the units. Similarly, although it would have been expected that more trade-qualified officers would be located in Beta Jail units as officer-inmate work gangs from Beta Jail performed the majority of maintenance around the facility, this was not the case. Rather, there was a reasonably even distribution of low tertiary level (trade) education throughout the prison. Table 7.1 demonstrates the demographic distribution by unit location.

Prisons in other Western societies, such as USA and Britain, have dealt with large numbers of staff and inmates for some time, what was problematic for New Zealand prison officers was the rapid increase in facility size and staff numbers. This combination of rapid expansion of the prison, influx of new officers and managerial separation into units had made it difficult for the whole group to be known anymore. The increased importance on the 'information hierarchy' (see chapter six) meant that, regardless of which script an officer was using, they required a high level of 'knowledge' about each other in order to: feel confident of their allegiance, trust that 
the other person could be relied on in emergencies, share gossip, socialise and so on. This level of knowledge was becoming increasingly difficult, however, with the introduction of unit management which then came to mean that social horizons were narrowed and focused on only knowing one’s own unit:

This is a big prison and there are a lot of prison staff I don't know and have no inclinations of wanting to get to know. I just don't have the time or the energy. It's only when you're working in close proximity, and that's when you make the effort.

- Male, 4-10 years service, Alpha Jail.

To illustrate this difference: in the early 1990s the officer group consisted of around sixty officers with a turnover of only one to two new officers per year. At the time of my research there was a staff of around 150 with an influx of around 30 officers per year. As such, it was no longer possible to know every officer. With the withdrawal into units, the simple act of transferring between units became problematic for officers as different units took on and developed work practices often at variance with those of both the total institution and the two jail sets:

\begin{abstract}
Unit management has created divisions between staff, you know. We support our unit rather than the officer corps so to speak. It's sort of created a lot of small groups, which is perhaps why when officers change units it takes, you know - not only is it having to learn sixty or so inmates' names, you're also having to learn all the idiosyncrasies of twenty staff that you've perhaps not had a lot to do with, and the new sorts of groupings there. And it does create quite a change whereas if you're operating as a corps, working together, those sort of changes wouldn't be as traumatic.
\end{abstract}

- Male, 4-10 years service, Beta Jail.

Therefore, it became advisable to only transfer to units which had known others or officers who seemed comparable to one's own work practices and values - leading to some pronounced demographic variations in each unit. These related in particular to the distribution of experienced officers and ethnic officers. 


\section{Table 7.1}

Unit Location: Demonstrating the Proportion of Each Demographic Category Within Each Unit Location

\begin{tabular}{|c|c|c|c|c|c|c|c|c|c|c|c|c|c|c|c|c|c|}
\hline \multirow{2}{*}{\multicolumn{2}{|c|}{ DEMOGRAPHIC CATEGORY }} & \multicolumn{16}{|c|}{ UNIT LOCATION } \\
\hline & & \multicolumn{2}{|c|}{$\begin{array}{c}\text { YELLOW } \\
(\mathbf{N}=15)\end{array}$} & \multicolumn{2}{|c|}{$\begin{array}{l}\text { ORANGE } \\
(\mathrm{N}=16)\end{array}$} & \multicolumn{2}{|c|}{$\begin{array}{l}\text { GREEN } \\
(\mathrm{N}=14)\end{array}$} & \multicolumn{2}{|c|}{$\begin{array}{c}\text { PINK } \\
(\mathrm{N}=12)\end{array}$} & \multicolumn{2}{|c|}{$\begin{array}{c}\text { LIME } \\
(\mathbf{N}=17)\end{array}$} & \multicolumn{2}{|c|}{$\begin{array}{c}\text { GREY } \\
(N=14)\end{array}$} & \multicolumn{2}{|c|}{$\begin{array}{c}\text { PURPLE } \\
(\mathrm{N}=14)\end{array}$} & \multicolumn{2}{|c|}{$\begin{array}{l}\text { EXCLUDED } \\
(\mathrm{N}=13)\end{array}$} \\
\hline JAIL TYPE & Alpha & (15) & $100.0 \%$ & (16) & $100.0 \%$ & (14) & $100.0 \%$ & $(0)$ & $0.0 \%$ & $(0)$ & $0.0 \%$ & $(0)$ & $0.0 \%$ & $(0)$ & $0.0 \%$ & $(0)$ & $0.0 \%$ \\
\hline & Beta & $(0)$ & $0.0 \%$ & $(0)$ & $0.0 \%$ & $(0)$ & $0.0 \%$ & $(12)$ & $100.0 \%$ & (17) & $100.0 \%$ & (14) & $100.0 \%$ & (14) & $100.0 \%$ & (13) & $100.0 \%$ \\
\hline \multirow[t]{2}{*}{ GENDER } & Male & (12) & $80.0 \%$ & (15) & $93.8 \%$ & (13) & $92.9 \%$ & (12) & $100.0 \%$ & (14) & $82.4 \%$ & (11) & $78.6 \%$ & (10) & $71.4 \%$ & (10) & $76.9 \%$ \\
\hline & Female & (3) & $20.0 \%$ & $(1)$ & $6.3 \%$ & (1) & $7.1 \%$ & $(0)$ & $0.0 \%$ & (3) & $17.6 \%$ & (3) & $21.4 \%$ & $(4)$ & $28.6 \%$ & (3) & $23.1 \%$ \\
\hline \multirow[t]{3}{*}{ ETHNICITY } & Pakeha & (11) & $73.3 \%$ & (9) & $56.3 \%$ & (10) & $71.4 \%$ & (7) & $58.3 \%$ & (7) & $41.2 \%$ & (7) & $50.0 \%$ & (10) & $71.4 \%$ & (11) & $84.6 \%$ \\
\hline & Maori & (4) & $26.7 \%$ & (5) & $31.3 \%$ & $(2)$ & $14.3 \%$ & (3) & $25.0 \%$ & (8) & $47.1 \%$ & (5) & $35.7 \%$ & (3) & $21.4 \%$ & $(2)$ & $15.4 \%$ \\
\hline & Pacific Island & (0) & $0.0 \%$ & (2) & $12.5 \%$ & (2) & $14.3 \%$ & (2) & $16.7 \%$ & (2) & $11.8 \%$ & (2) & $14.3 \%$ & (1) & $7.1 \%$ & (0) & $0.0 \%$ \\
\hline SERVICE & 0-4 years & (4) & $26.7 \%$ & (8) & $50.0 \%$ & (9) & $64.3 \%$ & (7) & $58.3 \%$ & (6) & $35.3 \%$ & (7) & $50.0 \%$ & (5) & $35.7 \%$ & (7) & $53.8 \%$ \\
\hline \multirow{2}{*}{ LENGTH } & 4-10 years & (5) & $33.3 \%$ & (5) & $31.3 \%$ & (4) & $28.6 \%$ & (4) & $33.3 \%$ & (6) & $35.3 \%$ & (5) & $35.7 \%$ & (7) & $50.0 \%$ & (6) & $46.2 \%$ \\
\hline & $10+$ years & (6) & $40.0 \%$ & (3) & $18.8 \%$ & (1) & $7.1 \%$ & (1) & $8.3 \%$ & (5) & $29.4 \%$ & (2) & $14.3 \%$ & (2) & $14.3 \%$ & (0) & $0.0 \%$ \\
\hline \multirow[t]{7}{*}{ AGE GROUP } & 20-24 years & (0) & $0.0 \%$ & $(0)$ & $0.0 \%$ & (0) & $0.0 \%$ & (0) & $0.0 \%$ & (0) & $0.0 \%$ & (1) & $7.1 \%$ & (0) & $0.0 \%$ & (1) & $7.7 \%$ \\
\hline & 25-29 years & (1) & $6.7 \%$ & (4) & $25.0 \%$ & (1) & $7.1 \%$ & (1) & $8.3 \%$ & (0) & $0.0 \%$ & (2) & $14.3 \%$ & (2) & $14.3 \%$ & (0) & $0.0 \%$ \\
\hline & 30-34 years & (3) & $20.0 \%$ & (5) & $31.3 \%$ & (3) & $21.4 \%$ & (1) & $8.3 \%$ & (6) & $35.3 \%$ & (1) & $7.1 \%$ & (2) & $14.3 \%$ & (5) & $38.5 \%$ \\
\hline & 35-39 years & (4) & $26.7 \%$ & (2) & $12.5 \%$ & (3) & $21.4 \%$ & (4) & $33.3 \%$ & (4) & $23.5 \%$ & (3) & $21.4 \%$ & (3) & $21.4 \%$ & (4) & $30.8 \%$ \\
\hline & 40-44 years & (4) & $26.7 \%$ & (1) & $6.3 \%$ & (5) & $35.7 \%$ & (3) & $25.0 \%$ & (2) & $11.8 \%$ & (4) & $28.6 \%$ & (4) & $28.6 \%$ & (2) & $15.4 \%$ \\
\hline & 45-49 years & (1) & $6.7 \%$ & (3) & $18.8 \%$ & (2) & $14.3 \%$ & (3) & $25.0 \%$ & (4) & $23.5 \%$ & (1) & $7.1 \%$ & (3) & $21.4 \%$ & (1) & $7.7 \%$ \\
\hline & 50-54 years & (2) & $13.3 \%$ & (1) & $6.3 \%$ & (0) & $0.0 \%$ & (0) & $0.0 \%$ & (1) & $5.9 \%$ & (2) & $14.3 \%$ & (0) & $0.0 \%$ & (0) & $0.0 \%$ \\
\hline
\end{tabular}


Cont...

\begin{tabular}{|c|c|c|c|c|c|c|c|c|c|c|c|c|c|c|c|c|c|}
\hline \multirow{2}{*}{\multicolumn{2}{|c|}{ DEMOGRAPHIC CATEGORY }} & \multicolumn{16}{|c|}{ UNIT LOCATION } \\
\hline & & \multicolumn{2}{|c|}{$\begin{array}{l}\text { YELLOW } \\
(\mathrm{N}=15)\end{array}$} & \multicolumn{2}{|c|}{$\begin{array}{l}\text { ORANGE } \\
(\mathrm{N}=16)\end{array}$} & \multicolumn{2}{|c|}{$\begin{array}{l}\text { GREEN } \\
(\mathrm{N}=14)\end{array}$} & \multicolumn{2}{|c|}{$\begin{array}{l}\text { PINK } \\
(\mathrm{N}=12)\end{array}$} & \multicolumn{2}{|c|}{$\begin{array}{l}\text { LIME } \\
(\mathrm{N}=17)\end{array}$} & \multicolumn{2}{|c|}{$\begin{array}{l}\text { GREY } \\
(\mathrm{N}=14)\end{array}$} & \multicolumn{2}{|c|}{$\begin{array}{l}\text { PURPLE } \\
(\mathrm{N}=14)\end{array}$} & \multicolumn{2}{|c|}{$\begin{array}{l}\text { EXCLUDED } \\
(\mathrm{N}=13)\end{array}$} \\
\hline EDUCATION & None & (3) & $20.0 \%$ & (3) & $18.8 \%$ & (2) & $14.3 \%$ & (1) & $8.3 \%$ & (2) & $11.8 \%$ & (3) & $21.4 \%$ & (2) & $14.3 \%$ & (1) & $7.7 \%$ \\
\hline \multirow{4}{*}{ LEVEL } & Low Secondary & (5) & $33.3 \%$ & (5) & $31.3 \%$ & (0) & $0.0 \%$ & (3) & $25.0 \%$ & (6) & $35.3 \%$ & (2) & $14.3 \%$ & (3) & $21.4 \%$ & (5) & $38.5 \%$ \\
\hline & High Secondary & (1) & $6.7 \%$ & (3) & $18.8 \%$ & (3) & $21.4 \%$ & (2) & $16.7 \%$ & (2) & $11.8 \%$ & (2) & $14.3 \%$ & (2) & $14.3 \%$ & (0) & $0.0 \%$ \\
\hline & Low Tertiary & (5) & $33.3 \%$ & (5) & $31.3 \%$ & (8) & $57.1 \%$ & (6) & $50.0 \%$ & (7) & $41.2 \%$ & (5) & $35.7 \%$ & (6) & $42.9 \%$ & (7) & $53.8 \%$ \\
\hline & High Tertiary & (1) & $6.7 \%$ & (0) & $0.0 \%$ & (1) & $7.1 \%$ & (0) & $0.0 \%$ & (0) & $0.0 \%$ & (2) & $14.3 \%$ & (1) & $7.1 \%$ & (0) & $0.0 \%$ \\
\hline \multirow{6}{*}{ JOB TITLE } & Probationary Officer & (1) & $6.7 \%$ & (2) & $12.5 \%$ & (4) & $28.6 \%$ & (4) & $33.3 \%$ & (4) & $23.5 \%$ & (3) & $21.4 \%$ & (2) & $14.3 \%$ & (5) & $38.5 \%$ \\
\hline & Prison Officer & & $40.0 \%$ & (9) & $56.3 \%$ & (5) & $35.7 \%$ & (4) & $33.3 \%$ & (5) & $29.4 \%$ & (4) & $28.6 \%$ & (3) & $21.4 \%$ & (3) & $23.1 \%$ \\
\hline & Instructor Officer & & $13.3 \%$ & (1) & $6.3 \%$ & (1) & $7.1 \%$ & (2) & $16.7 \%$ & (2) & $11.8 \%$ & (2) & $14.3 \%$ & (4) & $28.6 \%$ & (2) & $15.4 \%$ \\
\hline & Unit IC & (5) & $33.3 \%$ & (3) & $18.8 \%$ & (4) & $28.6 \%$ & (2) & $16.7 \%$ & (5) & $29.4 \%$ & (4) & $28.6 \%$ & (4) & $28.6 \%$ & (1) & $7.7 \%$ \\
\hline & Unit Manager & (1) & $6.7 \%$ & (1) & $6.3 \%$ & (0) & $0.0 \%$ & (0) & $0.0 \%$ & (1) & $5.9 \%$ & (1) & $7.1 \%$ & (1) & $7.1 \%$ & (1) & $7.7 \%$ \\
\hline & Missing Variables & & & & & & & & & & & & & & & (1) & $7.7 \%$ \\
\hline \multirow{2}{*}{$\begin{array}{l}\text { MILITARY } \\
\text { EXPERIENCE }\end{array}$} & Military Experience & (5) & $33.3 \%$ & (6) & $37.5 \%$ & (5) & $35.7 \%$ & (7) & $58.3 \%$ & (2) & $11.8 \%$ & (4) & $28.6 \%$ & (4) & $28.6 \%$ & (4) & $30.8 \%$ \\
\hline & $\begin{array}{l}\text { No Military Experience } \\
\text { Missing Variables }\end{array}$ & (10) & $66.7 \%$ & $\begin{array}{l}\text { (9) } \\
\text { (1) }\end{array}$ & $\begin{array}{l}56.3 \% \\
6.3 \%\end{array}$ & (9) & $64.3 \%$ & (5) & $41.7 \%$ & (15) & $88.2 \%$ & (10) & $71.4 \%$ & (10) & $71.4 \%$ & (9) & $69.2 \%$ \\
\hline \multirow{3}{*}{$\begin{array}{l}\text { ADDITIONAL } \\
\text { JAIL }\end{array}$} & No Experience & (5) & $33.3 \%$ & (11) & $68.8 \%$ & (13) & $92.9 \%$ & (11) & $91.7 \%$ & (10) & $58.8 \%$ & (8) & $57.1 \%$ & (10) & $71.4 \%$ & (11) & $84.6 \%$ \\
\hline & Additional Experience & (10) & $66.7 \%$ & (4) & $25.0 \%$ & (1) & $7.1 \%$ & (1) & $8.3 \%$ & (6) & $35.3 \%$ & (6) & $42.9 \%$ & (4) & $28.6 \%$ & (2) & $15.4 \%$ \\
\hline & Missing Variables & & & (1) & $6.3 \%$ & & & & & (1) & $5.9 \%$ & & & & & & \\
\hline
\end{tabular}




\section{Military Experience Within The Units}

The unit with the highest proportion of ex-military personnel was Pink Unit, one of the harmony units, where they constituted $58.3 \%$ of the complement. This was surprising given officers with military backgrounds, it could be assumed (see Soutar \& Williams, 1985; Morris \& Morris, 1963), would be less inclined to want to work in a unit with a majority of segregation type inmates, and even more so given that officers could request transfer to specific units. On the other hand, Lime Unit, whose officer group had the closest physical and social resemblance to a stereotypical 'army' unit, and whose inmates were generally serious offenders and ethnic gang members, actually contained the lowest proportion of ex-military with just $11.8 \%$ of their complement.

While one might have expected the ex-military culture to show influence in these units, this was not the case. Instead, ethnic solidarity took precedence over military culture. That is, both Pink and Lime Units were predominantly Maori officer groups with strong in-group associations; yet both units had very different inmate compositions and ex-military complements (Pink had high ex-military but harmony bonded inmates; Lime had low ex-military yet the more troublesome offenders). Therefore, something more than stereotypical male mateship created the composition of these units. The bonding feature instead was the ethnic clique which had developed in each, rather than any affinity for working with particular types of prisoners. It was as if the work associated with inmates was inconsequential - fellow staff members were what mattered.

\section{Additional Jail Experience Within The Units}

There were significant differences in additional jail experience of officers between the unit locations. Yellow Unit officers had a disproportionately high level of previous jail experience; Orange, Lime, Grey and Purple Units had average levels of additional jail experience; while Green, Pink and the Excluded Unit had officers with disproportionately low levels of prior jail experience. 
In Yellow Unit 66.7\% of officers had previous jail experience, which contributed to the perception that these officers were highly experienced. Thereby the security of the entire facility, the central task of that unit, was located with these officers. The disproportionately low level of additional prison experience within Green Unit, at only $7.1 \%$, meant these officers had limited institutional knowledge. The disturbances in this unit (see p. 87) were not unexpected by the general officer group, given the level of officer inexperience in it. Similarly, the Excluded Unit, which had two 'establishment phases’ during the course of my research (see pp. 83-84), experienced pronounced multiple-inmate incidents. Some officers interpreted this as an apparent reflection of their lack of experience, with few senior officers and their lack of additional institutional knowledge.

Finally, Pink Unit contained a clique which displayed high levels of dysfunctional and often inappropriate officer behaviour irrespective of whether it was viewed from the traditional or Head Office scripts - the Pink Unit script was entirely of its own making to suit the purposes and goals of its individual members. Indeed, several of the officers within Pink Unit were subject to Departmental Investigations during the research period. Given only $8.3 \%$ of this unit's compliment had additional prison experience, the general officer group took the view that Pink Unit officers' behaviour was a reflection of what happens when the prison authorities were prepared to disregard experience and institutional memory. The prison authorities had seemingly allowed it to be staffed by a group of officers that had been rejected by the wider officer body for their 'misbehaviour' and inability to be a 'good officer'. Rather than instruct these officers in an appropriate script, they had been allowed, instead, to put this into practise in a unit of their own without suitable experienced officer mentors.

\section{Ethnicity Within The Units}

Similar to the convivial goading between jails, there was frequent banter between the various units' officers. While this was generally playful, some perceived demographic differences, particularly ethnic, evoked suspicion and hostility within the larger officer group. For example, many Pakeha officers expressed an entrenched hostility towards the behaviours and dynamics within Pink and Lime Units, which were 
dominated by Maori officers ${ }^{158}$, and the separatist attitudes of these units' officers with their steadfast loyalty towards their own unit groups and Maori generally:

Racism here is being generated by staff. There are those out there that cause a lot of problems... Mind you, it's the same with some of the Pakeha guys, some of the Pakeha guys hate that bloody bunch (cliques), hate them! I don't hate them, I just don't like the way the unit is run. It's not them individually; it's just the Mafia style, all on their pedestals, that's it.

- Pakeha, 10+ years service, Beta Jail.

It was evident that the units with the greater proportions of non-Pakeha officers were the units with the strongest in-group loyalty and social atmosphere amongst the officers. In contrast, the units with the higher proportions of Pakeha officers were the least social and most atomised, namely Purple and Yellow Units. These had predominantly senior Pakeha officers ${ }^{159}$, seeking a quiet passage to retirement. Officers within these units were far more mechanical about their work arrangements, arriving on time to shift and leaving straight after handover.

The more 'sociable' units functioned on multiple levels to enhance their bonds, the majority of which actually resembled the traditional officer values and mores. For example, within such units officers were more likely to bring food for communal meal sessions and ensure 'spare' inmate meals were left in the warming drawer for officers on night watch. Further, officers would also remain after their shift for considerable periods of time to socialise with other officers within the unit. There were also more informal arrangements such as shift swapping and flexi-hours of shifts to accommodate each other's needs:

If you get on with them (your unit officers) and they like you, you should share (food), you know, it just becomes like family. If you feel comfortable with other officers, that's good, there's no conflict, you feel free, you know. It's important to get on with each other... every year you may have tricky situations where you're angry with something or something goes bad, and that's where you need the help from other officers, and that's only achieved if you get on with the

\footnotetext{
158 The perception of ethnic dominance in Lime Unit was demographically supported since $25 \%$ of Maori officers at the target institution were located there. Indeed, Lime Unit had the lowest proportion of Pakeha officers at only $41.2 \%$ of their complement. Pink Unit was also predominantly Maori, however, this is not shown in the questionnaire data as the majority of this ethnic clique were nonreturnees.

${ }^{159}$ Purple Unit contained 71.4\% and Yellow Unit 73.3\% Pakeha officers.
} 
officers and sharing meals and having a laugh together after shift is important for getting on.

- Male, 0-4 years service, Beta Jail.

These predominantly non-Pakeha units were more likely to be involved in inter-unit sports games and challenges, or involved in inter-prison and external sporting events. 'Outside the wire', these unit officers socialised extensively with one another, such as going to each other's homes for parties and excursions to local pubs and clubs. A couple of these units even had 'unit social clubs' with a bank account in which they would contribute money each payday. They could then draw on this to fund social gatherings or give a portion to one of the unit members who may be experiencing hardship and so on.

Socialising exclusively with other officers was extremely common amongst those who lived in the village (many of whom were also in the clique units) - on Friday and Saturday nights village officers would wander around the village streets until they found the 'garage' the evening's party was at and join in ${ }^{160}$. In the past, attendance at such parties was common for all members of the officer body as they were all 'connected' to the village. However, with the increased fragmentation of the officer group, these parties were now the exclusive domain of village officers, thus predominantly clique officers and ethnic officers (as these officers were more readily accepted by the cliques, even if not members of them). This intense in-group socialisation effectively reduced these officers' external associations with other work colleagues while increasing their insularity. Officers came to increasingly rely on this social network as they lost touch with pre-prison friends and acquaintances, learning to justify their insularity as a feature of the job. It also reinforced their growing suspicions of outsiders, including 'outsider' officer groups:

You've got groups here, they come to work and say 'prison's not a place to work, it's a way of life'. You come here, you work here, and you socialise with the workmates - and literally you're living in this pocket 24 hours of the day. You have this incestiousness (sic) going on all the time.

- Female, 4-10 years service, Alpha Jail.

\footnotetext{
${ }^{160}$ Garage parties are a working class New Zealand tradition, particularly amongst non-Pakeha groups. Typically, the garage is bare apart from chairs, a 'beer fridge', an old stereo and guitars.
} 
In this way, a feature of prison life which had brought officers together in the past had now become another way of dividing them.

Indeed, even as the prison village was being dismantled towards the end of this research, village prison officers were constructing their own village-like living conditions by purchasing and renting homes in direct vicinity of each other. Some even relocated the original prison homes to these areas, living in them themselves or renting them to fellow officers. Formally village life was deemed unhealthy and no longer required for prison security, but as with the epaulettes, this feature of their life was too important, so informally it persisted, particularly amongst the ethnic and clique officers who had resided there. In this way, they were able to prolong the exclusivity and activity of this set of officers.

\section{Age, Length Of Service And Job Title Within The Units}

'Age' was not generally considered a defining characteristic within the target officer group. Regardless of age, the vast majority of officers had been married at least once, had a minimum of one child and had held at least one other job long-term. A more important aspect was 'years' related to length of service rather than age. When officers spoke of being 'old' or 'young' it referred to longevity of service.

The officer group held firm beliefs about how length of service should be arranged across the prison and hence within specific units. For example, officers asserted that there should be a greater number of senior staff in Yellow Unit, as this was the communications unit which oversaw the safety and security of the entire institution. Indeed, Yellow Unit had the lowest proportion of 0-4 years service officers at 26.7\% and highest proportion of $10+$ years service officers at $40.0 \%$ of their complement.

Of the $10+$ years service category, 55\% were located in just two units. While officers were correct in the apparent dominance of longer service officers in Yellow Unit, which had $30 \%$ of the total $10+$ years category, the second unit with a high proportion of longer service officers was actually Lime Unit with $25 \%$ of the $10+$ years category. This meant many inexperienced officers were concentrated in the other six units. 
The high proportion of 10+ years service length officers in Yellow Unit supported the officers' perception that this was a vital unit requiring experienced staff. Officers were comfortable with this arrangement. It also demonstrated that, for officers at least, longer length of service was a reflection of ability and trustworthiness. On the other hand, the combination of a high proportion of non-Pakeha and 25\% of the $10+$ years length of service officers in Lime Unit had the effect of reinforcing the exclusiveness and power of this group across the officer body as these were the very officers that it was most difficult to gain acceptance from. As one officer, not a member of this unit, explained:

It's probably no accident that they're all darkies. I don't think there's any, if there is there's very few Europeans working in there. Be it right or wrong I couldn't say, but it works for them and they're all happy and their unit runs well. They're all SPOs too. They've sort of come through together... they've all sort of come up together and grown up together kind of thing in the job. That's probably why, looking back four or five years ago, they probably would have all been juniors running around, and everyone's thinking 'fuck what are we going to make of these guys' and they've just come up and fallen in together, good planning though from a unit perspective, they've said 'this is how we're going to work' and they've done it.

- Pakeha, 4-10 years service, Beta Jail.

A major concern amongst the general officer group was that there were high proportions of probationary staff in the medium security units ${ }^{161}$. Officers maintained that Orange and Green Units should have more senior staff to ensure control. Indeed Orange Unit and Green Unit had $50.0 \%$ and $64.3 \%$ of their respective officer compliments as 0-4 years service officers. While officers were critical of management for 'allowing' this situation to develop, in reality the distribution had occurred because the majority of senior officers had chosen to locate to Yellow and Lime Units. Numerous complaints had been voiced to management by officers regarding the situation but without result. This meant that the only means officers had to make their point to management was for the system to fail, which reflected the gulf that now existed between these two groups:

\footnotetext{
161 The lack of experience within Orange and Green Units was particularly important as they were supporting units. When an incident occurred, Orange and Green Units' officers had an arrangement to supply available officers to assist the adjacent unit. Remaining officers locked down their unit's inmates and followed promptly to assist.
} 
The staff need to see the boss, they need to see that he's around, the inmates also... that (Green Unit) riot was a good example, that will happen again. It will be a (Beta Jail) unit though. They'll loose a unit. It's not a good thought. You wouldn't contain it. By the time staff sorted (it) out, they'd have it burnt to the ground. It wouldn't take them long. Maybe then they'll listen to us!

- Male, 4-10 years service, Alpha Jail.

Indeed, the system did fail with several notable Orange Unit inmate escapes and the Green Unit riot. Officers asserted this made three clear points about upper management: they had no right to place such low service level officers in this position; by doing so they had not only endangered these officers but endangered the entire officer group; and they had only allowed this situation to develop because they were more concerned with saving money ${ }^{162}$ than the safety of officers or the institution. This only further reinforced the rigid line that now existed between these two groups.

Despite these assertions, however, it was also clear that the varied demographic groupings had emerged in the units because of the manipulation of elective transfer requests by officers. If this reflected a failure of management to be more directive, it also reflected the officers' choices. Over time these groupings became self-fulfilling as only certain types of officers would elect to transfer to particular units:

\begin{abstract}
There are some units that I wouldn't apply for.... Like [Lime Unit], they've got their own sort of rules, you know. I wouldn't want to fit into that, the way that they run their unit, I wouldn't want to be part of it. So yeah, I wouldn't apply for their unit simple as that (pause). I would find it quite frustrating to work in as I've got my own morals and my own standards which is a little bit different to what they do and the racist side of it sort of thing. I've got the wrong colour skin.

- Pakeha, 0-4 years service, Alpha Jail.
\end{abstract}

As this extract demonstrates, the combination of the demography and resulting work culture of the dominant scripts of a given unit were now the determining factors of whether an officer would want to relocate there or not. Had management attempted to effect this development they would have been meet with resistance and resentment, as

\footnotetext{
162 Officers argued the prison authorities were saving money by having the minimum number of staff rostered on when, given the low level of experience, it would have been preferable to have additional staff members on duty to aid the rostered staff. Further, officers purported the apparent move to discourage senior officers to stay employed was a reflection of cost cutting as junior staff had considerably lower salaries.
} 
was the case for Pacific Island officers who were unable to transfer. In this way, prison officers, through selective transfer requests, were unwittingly contributing to their own pains of occupation by actively concentrating longer serving officers and ethnic officers in particular units. By doing this, they allowed ethnic officers to gain power through association of numbers and prevented the effective transmission of the traditional officers' script which still dominated many of their expectations of officers’ informal behaviour.

\section{The Consequences Of Unit Divisions}

Experienced officers could see the changes in social dynamics of the officer corps as staff began retreating into their units. For example, the common meeting area for officers had been the staff facility building. For as long as officers could remember they would gather there whenever they had a free moment during shift, before and after shift, even having network meetings and social functions there on the weekends and special holidays. However, with the rapid expansion of the prison, officers were electing to remain in their respective units during designated break periods:

\begin{abstract}
They (management) gave us a staff facility. When I first started it would be buzzing eh. They'd be laughing, sharing food with each other, making jokes, talking about their families and stuff, and all the problems with the institutions. Oh the camaraderie! But that all changed when we got the units. Now you go over there, you look at the place, it's very drab, there's nothing there. People don't want to go over there any more. It's not user friendly, why sit over there? They moan about their bosses (Unit ICs), the workload, the other staff and about management. They're not even listening (to each other). And there are officers just sitting there by themselves, not in conversation at all! \{my emphasis\}
\end{abstract}

- Male, 4-10 years service, Alpha Jail.

As this extract demonstrates, the previous homogeneity and unity of the prison officer group had indeed changed as a result of the 1990s policy changes. Where in the past officers actively sought each other's company for moral support, morale enhancement, fun and camaraderie, they may now actively avoid officers from other units. It also meant that the easy social acceptance that there had previously been 
between officers was a thing of the past. As illustrated above, going to the staff facility might now mean them just sitting on their own.

Indeed, it was recognised that the convivial rivalry had turned into polarised antipathy and opposition:

I find it amazing eh, we're all prison officers, I find that incredible and I don't understand why there should be rivalry because at the end of the day we're all doing the same job? I don't see that there's any difference, you know, 'you want to work at [Alpha Jail] (then) work at [Alpha Jail], if you don't, don't'. Why make a sort of rivalry thing, its crazy? It's sad actually when you think about it. We're all the same really. We're all one big unit - one big family at the end of the day. But that's not how it plays out... it's all us and them.

- Female, 0-4 years service, Alpha Jail.

The simple group allegiances which had existed had become multi-layered, characterised by fragmentation rather than cohesion. One officer ${ }^{163}$ described the combined effects of regionalisation and unit management as 'an alteration of the family unit'. That is, previously, the officers at a given officer's prison were their brothers and sisters; and officers at other prisons were their cousins. As such, bonds were close throughout any given prison, and a close community relationship extended across the national prison officer group (see Figure 7.1).

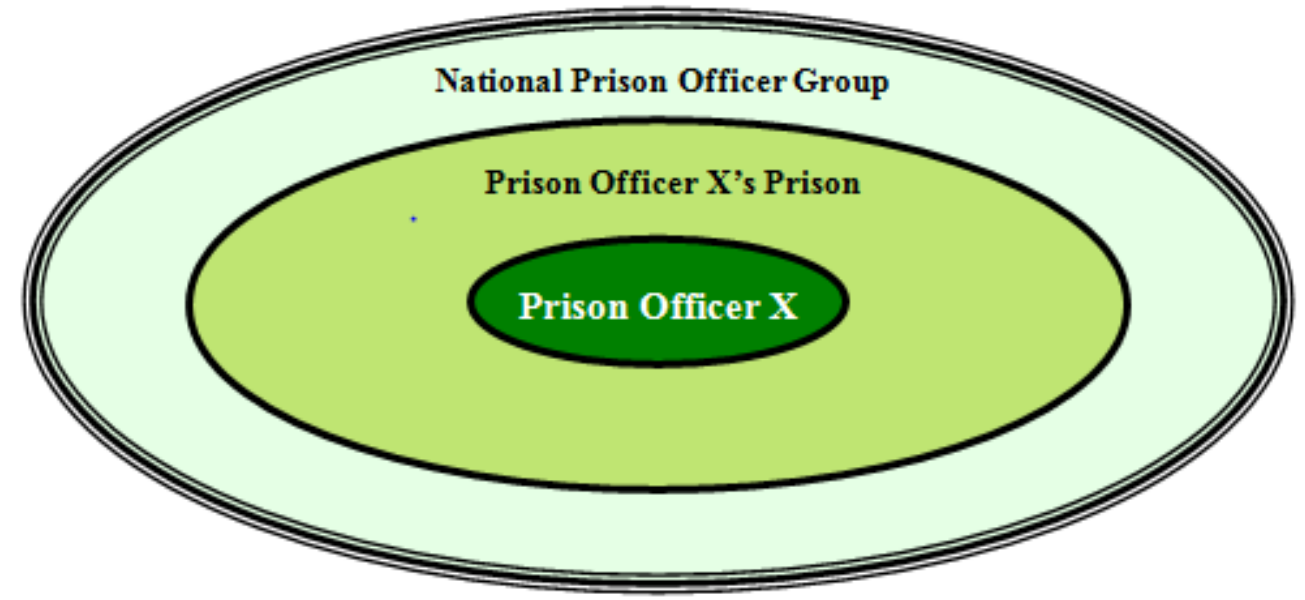

Figure 7.1. Diagrammatic representation of a given prison officer's group allegiances prior to regionalisation and unit management.

${ }^{163}$ Male, 4-10 years service, Beta Jail. 
Following regionalisation and unit management, a given officer's direct group reduced in size while at the same time increased in layers. In this way, an officer's unit officers were their brothers and sisters; the officers at their jail were their cousins; the officers at their prison were their second cousins and so on. In this way, associations intensified at the unit level, but became weaker and more remote outside of this (see Figure 7.2).

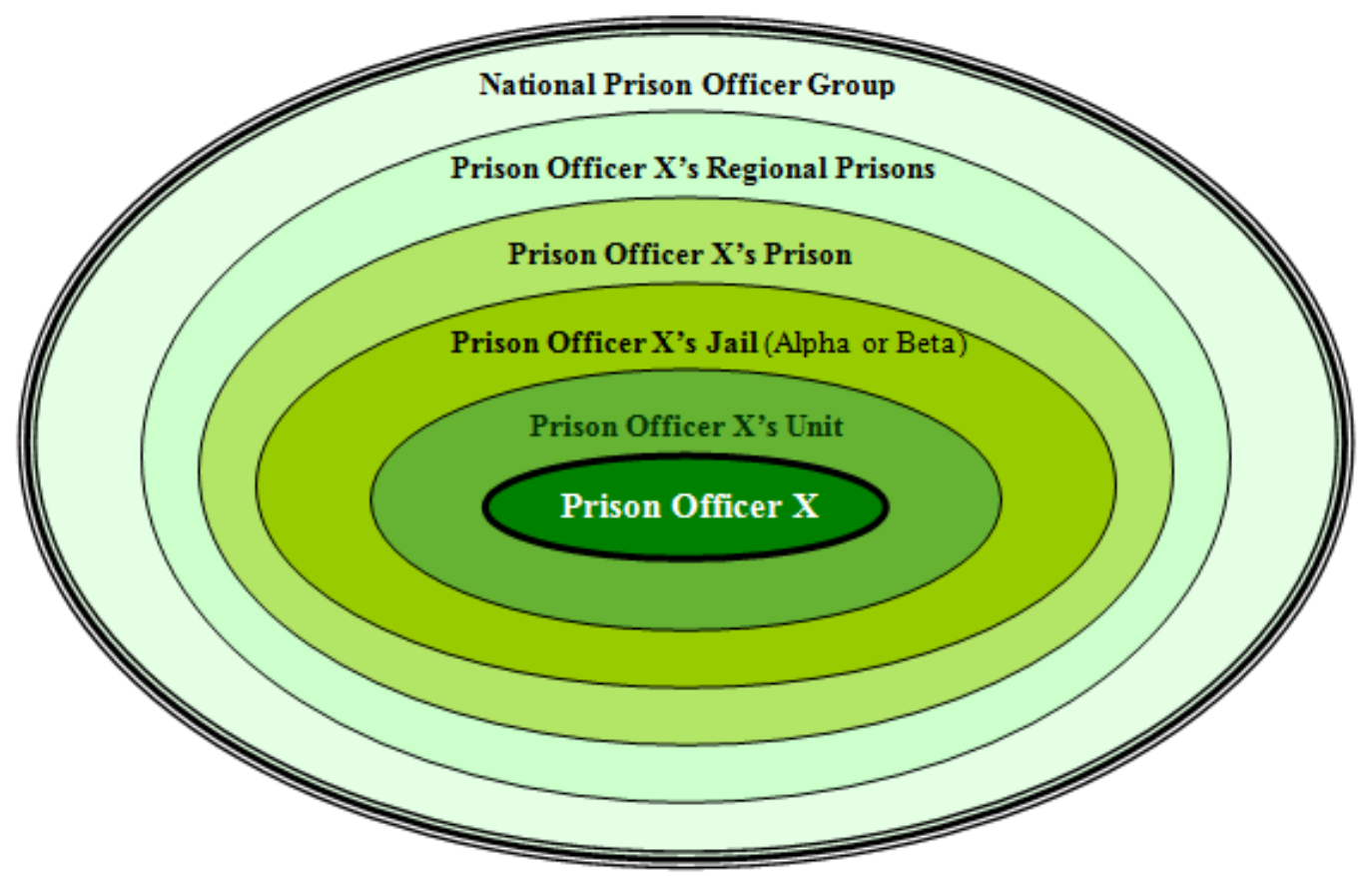

Figure 7.2. Diagrammatic representation of a given prison officer's group allegiances after regionalisation and unit management.

Goffman (1959) referred to this as clan segmentation. He argued that in large groups clan segmentation is necessary to simplify social encounters. Historically, the entire prison officer group had been one team regardless of which unit or wing the officer worked in. Clan segmentation was established through rank. With the removal of the rank structure, rapid increase in facility size and introduction of unit management clan segmentation re-formed whereby each unit was now a distinct clan or team. In such circumstances, bonds with other colleagues are weakened. Officers were well aware of this separation from officers of other units:

The main change was when we became the units. It's not quite the same feeling. You're separated because it's more of a business now. Its very business oriented. It's run by businessmen - all the way down the line to the shop floor. Whereas before it wasn't a business - it was about people.

- Male, 10+ years service, Alpha Jail. 
They were equally aware that unit locations had acquired, and were developing, their own variations of prison officer culture, but also that there was little that could be done to change the situation that had developed:

It's like some units have visits and they're permitted to bring in food or something, which is totally not permitted but is part of the culture of the unit, you know... there are operating guidelines (PPM), they operate at the local level, which is a bit of a pain because it brings inconsistency in I think... so what do you do, do you fight it, do you support it, or do you choose to ignore it, I don't know? \{my emphasis\}

- Male, 4-10 years service, Alpha Jail.

It would seem that 'what officers chose to do about it' was to 'ignore it', not elect to transfer to particular units that had a script they had no affinity with and focus instead on their own unit. This fragmentation and competition was evident in everyday interaction. For example, it now became possible to hear an officer say things which would previously have been unthinkable: 'not our jail, not our problem', even 'not our unit, not our problem'. It would take serious incidents, such as the riot in Green Unit, to revive allegiances and bring about a temporary display of collegiality, regardless of associations:

Oh it was great during the riot eh, [one regional prison's name] people came, [another regional prison's name] came over, you know, that comes back to the loyalty thing doesn't it. (We are) there for each other. There was talk when [name of a non-regional prison] was going down that we should send some guys up but then it ended.

- Male, 0-4 years service, Beta Jail.

Such events provided a rare glimpse of the 'unity of old' within the officer set. As a means of indicating the group was a unified whole, officers, even those not directly involved, would refer to themselves as 'the Green Machine, ${ }^{164}$. This was a term frequently bandied about during discussions of this event. During the course of the double shift in which the riot occurred, and for several weeks to follow, there were no references to 'units', cliques or any other group affiliations. Rather, the officers were one group which had united and triumphed over adversity in the face of perceived disorganisation and mismanagement from management.

164 'Green' as that was considered the dominant colour of their uniform (brown being the secondary colour). 
Typically, groups are bound by what they have in common (see Crawley, 2004). Following the Green Unit riot, an intensified opposition to management bound the officers across the prison closer together. This arose from management's perceived lack of leadership during the riot - and their denial of any responsibility for it afterwards:

\begin{abstract}
The whole thing could have been dealt with within about an hour and it dragged on and on and ended up taking about seven or eight hours. The staff were really ropable, they're all here, all itching, not so much itching to have a bit of a biff, itching to get in there, have it over and done with, locked down, dealt with... (but) they couldn't even organise a C\&R team to stand by and get ready, 'don't worry - hang on a sec, don't worry guys - hang on a sec’. That happened about three times and when you're going into a riot and you're all psyched up to go in and nail some guy on the floor and get him restrained and remove him safely out of the area with minimum damage to your fellow staff members, and you're stood down? It's an adrenaline thing, you just can't maintain it for eight hours, you gotta get in there and do the job. Its all in your sight, its all you're focused on - you can't stay focused for that length of time. Everyone was just so wild and angry.
\end{abstract}

- Male, 0-4 years service, Alpha Jail.

What made the riot and its aftermath even worse was the absence of the majority of senior and non-Pakeha officers. They were not present that weekend as they were attending a National Inter-Prison sporting event out of the region, which meant that the riot squads were predominantly made up of probationary and 1-2 year service officers. While low length of service officers were trained for such events, their lack of experience in serious incidents meant they were ill prepared, placing all officers, indeed the jail, in a precarious position. Despite this, the event and their willingness to act enabled these officers to become known to the wider officer group. They then accredited them with status, recognition and (at least temporary) acceptance that was given to any low service length officer who displayed 'the right stuff' in a serious incident:

Interviewer: You haven't been here that long, yet I've heard a lot of good things about you - how do you think you earned your way into that category so quick?

Officer: ... I put it down to that incident the night we had the trouble in the unit here $^{165}$. I think people saw me react to it and I seemed to (get a) better response from the inmates that saw what happened. I heard from the staff and amongst the compound that I handled it okay... (Its) interesting the old rumour mill working

\footnotetext{
${ }^{165}$ A multiple-inmate disturbance in Excluded Unit that caused the lockdown of the entire facility so that officers could assist the given unit reassert control over a group of intoxicated inmates.
} 
here, I guess they (officers) know I'm a joker but when the shit hits the fan I'm there. I mean that's why we're here, it's not what we're here for, but that's the time we truly get tested. You know if I'd done something slightly different I could have had the reputation of being a fucking egg and that would have stuck with me for the rest of my days. Yeah, people (officers and inmates) are very judgemental around here. There's a lot of good grudge holders. I was talking about going to work at [Alpha Jail] and a couple of officers said 'we're keen to have you up there, you'll do a good job' and I said 'you don't know me, you've never worked with me' and they said 'oh no, we've heard'. I was just lucky.

- Male, 0-4 years service, Beta Jail.

Such unifying and reputation endorsing events were rare, however. On a day-to-day level, officers needed to attain a sense of cohesion in some other way. This was difficult to achieve with the weakening of prison officer culture across the institution. Instead, they usually had to look for this at the unit level where social relations could be manipulated to produce specific demographic and social groupings. In this way, rather than reducing the effect of the historical prison culture, the new prison structure merely altered the nature of social relations within the wider group, locating the culture in ever-smaller groups while paradoxically increasing its influence and establishing unit specific variations of it.

As we have seen, it was difficult for the wider prison group to acculturate recruits within a broad officer culture. Instead, new officers were limited to the experience and induction by officers within their given unit. Even long serving officers recognised that power now lay at the unit level rather than the prison level. As group allegiances and perceived demographic distribution contributed to the grouping of staff within the units, and each group emphasised varying aspects of the prison officer culture, the unit to which a new recruit was posted played a significant role in the development of the type of officer this individual would become and the likely associations that would ensue - further perpetuating this new problem.

Structural effects on prison officer relations were not only imposed but also came from within the officer group. The industrial dispute of 1996 contributed significantly to the loosening of categorical loyalties and the retreat into unit allegiances. The POA officers had been on strike for fifteen days while PSA officers continued to work (see pp. 132-133). POA officers were generally understanding of the PSA officers as they were not legally able to join the picket line. What they did consider problematic, 
however, was the high number of officers who 'crossed the picket line' by changing membership to the PSA to avoid the industrial action:

\begin{abstract}
Its not PSA versus POA, its the people who jumped ship. When the POA looked like they were going to be more staunch than the PSA, we actually might end up on the picket line, they were saying 'yeah yeah let's do it'. We had our final vote and when it came down to it, the day before we all got suspended, they suddenly showed up to work as members of the PSA. We don't hold that against the PSA themselves but those guys that were all for it and suddenly they were crossing the picket line, yeah there was resentment from it. One or two were my friends. I still remember that, you know. That showed you the character of them. Came time to stand up and be counted, they all jumped ship. They were the ones that copped a lot of that crap on that picket line as they were cruising past. The PSA members that drove to work and that, they weren't part of our argument and part of our fight and we left them alone, you know. It wasn't their fault. It wasn't their contract. But it was those that were playing, jumping both sides of the fence that cause resentment.
\end{abstract}

- Male, 4-10 years service, Alpha Jail.

The already tenuous bonds at the prison and jail levels were further loosened as it was apparent that many officers were no longer prepared to undergo sacrifice and hardship for the good of all:

\begin{abstract}
After we had that last industrial action there was two totally separate groups because one lot was on strike and one lot wasn't. That sort of created a bit of friction.... There were those that didn't go on strike that were sympathetic to the cause, and those that did nothing to help themselves, who fucking ignored those who were on strike. Those that were wankers got fucking treated as wankers. I mean some people will still have an attitude (against) those that did it last time because they won't let it go or whatever. We'll never fucking forget that shit... you think you know somebody!
\end{abstract}

- Male, 4-10 years service, Beta Jail.

Increased recruitment of officers with perfunctory work and relationship attitudes further cemented this retrenchment, as this style of officer was demonstrably individually focused. For example, many of these new recruits were choosing to join the PSA union because of the perks of membership, such as the availability of PSA owned low cost holiday rental homes, rather than any philanthropic ideals about collective benefits.

Crawley (2004, p. 40) noted that in the presence of hostility from 'outsiders', officers as a group would take a confrontational stance and "circle the wagons". This 
technique of 'self-preservation' explains the retreat into unit groups as new 'insiders' (and some existing officers) came to be viewed as just as much a threat as 'outsiders' had been in the past. The investigations of Mangaroa and Crawford Prisons in the early 1990s (see p. 128) led to many staff at the respective prisons being sanctioned and dismissed. A series of internal investigations swept through all New Zealand prisons in the ensuing years. As a result, officer groups felt they could not trust any 'unknown' officer to abide by the informal rules and secure the safety of the group:

\begin{abstract}
We still have the big network, oh, it's probably true to a certain extent but it's not as prevalent as it was a few years back leading up to the episodes at Mangaroa and Crawford. You know, where they actually exposed certain factions of staff who were supposedly involved in an almost gang beating and drug trafficking and that. I think that was a big wake-up call to staff round the country eh. That some behaviour is not acceptable and if you're narked upon by a fellow staff member or inmate - watch out, your job could be on the line! So you didn't, you know, you stuck more to your own more.
\end{abstract}

- Male, 4-10 years service, Beta Jail.

Hence the 'circling of wagons' - but in ever decreasing circles:

You probably felt a lot safer in the job back then because you knew the guys a lot better, whereas now you're not too sure who you've got on with you.... The longer you're working with a person the more you know about them and the safer you feel with them.... Now it's forever new staff coming on. It just takes that much longer to meet them, see how they work, and things like that.

- Male, 10+ years service, Beta Jail.

Overall, then, within the units, demographic groupings began to emerge which led to divergent practices, occupational cultures and scripts. As officers 'circled their wagons' in the face of uncertainty and instability from both the prison authorities investigations and the new threat of unknown 'insiders', power came to rest at the unit level. It was this power, and the absence of management's watchful eye, that enabled the formation of unit specific cliques. 


\section{Clique Power}

As their numbers and importance increased, this sense of empowerment within the units provided the momentum for non-Pakeha officers to divide into two distinct cliques. This division marked the disintegration of the Maori network - the official platform for ethnic solidarity. Instead, the Bros emerged in Lime Unit and the Black Mafia in Pink Unit. The Bros developed during the early 1990s when the majority of these members joined the service. As they were the minority ethnic group at that time, they associated regularly together and through manipulation of transfers, got themselves placed in one unit. As the Unit Manager and Unit IC were also members of the clique, its conduct was left unchecked:

\begin{abstract}
Well let's look at [Lime Unit], most of the staff are all darkies. They're probably the cliquiest unit of the whole jail. They are just such a tight unit. But breaking into their circle is a little bit different. Different things go on. But the reason that their staff have been the way they are is because [Unit IC's name] has had them together for so long. They work to his rule. And he's one of the boys, if you know what I mean. He's the boss but he's one of the boys. And they're all SPOs. And they're all big buggers and that, good guys, they rip each other off to the max, give each other absolute death all the time. But at the end of the day they know that [Unit IC's name]'s the boss and what [Unit IC's name] tells them to do they'll just do it because that's where they hold him. And that's why they've stuck together for so long, other bosses could get that too if they're strong enough to stand up to the boss at the top and say 'these are my staff, you're not taking them' then the bosses will back off like they did with [Lime Unit]. That's where the staff become tight cos they know the boss wants them. That's why [Lime Unit] runs like it does, runs like the breeze. I mean they've probably got the worst inmates there as far as the ratbags go, the heavies and what not, but for the crims over there, they don't have that many incidents and its because of the staff they've got, that physical presence, and because all the inmates know you couldn't step out of line with any one of those staff members because you'd have every single one of them down on you like a bull.
\end{abstract}

- Maori, 4-10 years service, Beta Jail.

The formation of the Black Mafia took place on a more ad hoc basis. Anecdotal accounts suggest that certain members of the Black Mafia were not interested in the strict rules and hierarchy that the Bros became renowned for and hence formed a breakaway renegade group. Allegedly the Bros were happy for this group to withdraw to another unit as they were not being discrete about what they were doing and might have attracted the attention of the prison authorities. 
Both cliques at the time of my research could be categorised as predominantly nonPakeha, although membership was not exclusively 'brown'. Generally members were within the 4-10 years service category and few new members were accepted unless they met strict criteria. They had to be: non-Pakeha; physically commanding; willing to participate in the groups' deviant behaviour (see below); and had passed a series of rigorous officer initiated character tests to demonstrate unquestioning loyalty to the clique and (in the case of the Bros) obedience to the clique hierarchy and rules above those of the prison. Placement in a clique unit did not guarantee acceptance. Indeed, clique members would attempt various strategies to 'encourage' undesirable officers to transfer out of their unit at the earliest possible convenience:

The number of white men who have passed through those two units (Pink and Lime) who have come out of there shell shocked by the treatment they've had, not everybody's like that, not all Maoris (sic) are like that.

- Pakeha, 10+ years service, Beta Jail.

The cliques preferred to 'test' potential members while they were attached to other units, then assist with transfer applications to their unit if the officer was thought acceptable. These started with a willingness to be associated with the clique out of duty hours, ability to consume large volumes of alcohol and willingness to follow any order (ranging from performing childish pranks to physically disciplining inmates):

... they might have to do something for the other members of that clique, you know, to be accepted. Instances where staff have been involved in mildly criminal activities such as theft, they're in that clique and were asked to do certain things that they weren't happy with. To maintain their presence in the clique they had to do it or turn a blind eye to whatever it was they were asked to do.

- Male, 4-10 years service, Beta Jail.

To some, the prospect of acceptance from a clique (and benefits of membership such as a sense of 'brotherhood') was so appealing that they could 'temporarily lose perspective'. One officer disclosed he had spent thousands of dollars on alcohol and entertainment for one of the cliques, only to be ultimately rejected by them. 
The two cliques had different structures which everyone knew about. The Bros had a strict hierarchy that was based on physical prowess and length of service in the clique. They had clear channels of communication and power and an almost military precision in organisation (paradoxically given they had a very low ex-military complement). An affront to one Bro was an affront to them all and they would react accordingly. Few dared to challenge them, neither inmates, nor officers nor management. If they wanted something they got it, if they disagreed with something it ceased. The Black Mafia was less structured (verging on disorganised) and was based more on identifiable kinship relationships within the group. As such, they were neither inclined to react en masse nor was retaliation (if an officer had offended against them) certain, making them a less effective 'gang'. Both cliques had presiding 'kingpins' though, whom the respective groups obeyed unquestioningly:

We've got a kingpin in each unit, depending in which clique you're looking at. I
mean our clique here, they have got a little hierarchy. Doesn't matter where in
the jail you are everyone knows the hierarchy. - Clique member.

Being a member of a clique had a high cost in terms of time commitment to the group. Participating in group activities extended into all facets of the officer's life (professional and personal). At no time could such an officer 'switch off' to the needs of his clique 'brothers'. For their unconditional loyalty, however, clique membership had its advantages. A primary benefit was the familial bond within the group. On acceptance, one was reciprocally bonded with the fifteen other clique members in the unit - the ultimate in male mateship and camaraderie:

My workmates, it's very tight knit. I can call on them 24 hours a day, ring them up and say what I've done, any situation. Regardless if its here or at home, anywhere. And that's something that's not easy to build up eh. Even my wife says 'how do you get that?' and I say 'it's the things we've been through, we can trust each other'. Now that's camaraderie, very tight knit and I love it.

- Clique member.

As an example, a clique officer wished to attend a funeral but was concerned (as the deceased's family did not want this person to attend). As a demonstration of support and protection, the majority of the clique also attended the funeral (arranging cover 
for their unit from non-clique officers), standing menacingly around the officer like a guard of honour to prevent any untoward confrontations.

Importantly, the cliques also seemed to have an ability to gain advantages that were beyond other officer groups. They were able to manipulate their environment and those within it. As an example, the Bro 2IC stopped smoking (nicotine) and decreed that their unit would now be 'smoke-free', at that time an unprecedented policy in a New Zealand prison. Any officer or inmate who then wished to smoke in the unit had to do so discretely, dispose of their cigarette butt directly into the drains so as not to pollute the grounds, and under no circumstances smoke near the guardroom where the 2IC resided. All complied. Throughout the rest of the prison both inmates and officers were prolific cigarette smokers, yet in this unit, only a handful continued and were constantly reminded by the others (inmates and officers) how 'filthy' their habit was.

With the spatial separation of ethnic cliques into units, distinctive behaviours, such as communication style, symbolic gestures, mores and language began to emerge as their characteristics. Establishing observable group membership behaviours provided strong in-group identity, demonstrated out-group status to non-clique members and set in motion an overt us-them set of relations between the two cliques. The autonomy of both cliques had allowed them to rewrite their respective prison officer scripts to legitimate a variety of dishonest behaviours - which non-clique officers had significant reservations about. For example, the cliques extracted what they wanted from others (officers and inmates) through the threat of physical recriminations, they imposed 'taxes' on inmates' goods coming into the unit ${ }^{166}$, physically controlled inmates and intimidated non-clique staff whom they perceived as threatening to the harmony of their unit arrangements.

However, the two cliques had very different motives for their dishonesty. The Bros participated in such behaviours to reinforce group solidarity and thereby maintain an efficiently run unit. Although not supporting such tactics, most officers could

\footnotetext{
166 The tax system was a forced 'negotiated' trade between officers and inmates, typically invoked in regards to accepting certain property into the institution. As an example, it was against the formal regulations for visitors to bring goods in for the inmates during visits unless the items had prior approval from the Unit Manager or Manager Custody. However, if an inmate's partner brought a cake in during a visit, it could be accepted, provided that the inmate allowed a few slices to be taken out by the visits officer (and given to the shift officers) as 'tax' for the transaction.
} 
understand the logic of such behaviours and as long as these were contained within the unit (or it was beneficial to them - the Bros, for example, were frequently the officers called into other units to 'quell' potential disturbances), they were not perturbed. The Black Mafia, on the other hand, used such behaviours for the exclusive benefit of themselves. No other officer could see the justification for this. To illustrate this difference, during my research a Black Mafia officer was under investigation for stealing boxes of toilet paper from the prison stores (which he allegedly took home as a supplement to wages). Other officers viewed this as inexcusable. Had this been done by the Bros, a group (rather than an individual) would have used 'standover tactics' to intimidate the inmate assistants in the stores to give them the boxes of toilet paper (being up front as per the traditional prison culture script rather than surreptitious, although, at the same time, ensuring that management would not become aware of the activity). The toilet paper would then be used in the unit (rather than taken home), allowing the money in the budget that would have been used on toilet paper to be used for other purposes — such as the purchase of additional food rations — thereby leading to happy inmates and a smooth running unit.

Although non-clique officers did not generally support or approve of such behaviours by the cliques, they had little influence over a specific unit's activities. They did, though, have to be wary of their colleagues who acted in this way. One officer recounted a serious incident of standover tactics within a clique unit while he was seconded there. However, when he raised the issue with the Unit Manager he was told to 'keep quiet' and was promptly transferred away from the unit.

Another behaviour which the officer body did not approve of was the co-option of inmate élites to maintain control within the unit (an informal 'Building Trader' or ‘Turnkey’ system, see Marquart \& Roebuck, 1986). A number of the Black Mafia were 'preoccupied' during shift time (on personal activities) and therefore relied on inmates to maintain the unit routines:

I've seen staff who used those kingpins and their cronies to keep a wing quiet, to deal with the trouble. Now that is a no-no. As soon as you allow this type of control, what are you doing at work? You may as well hand them the keys because you don't have control because they're running the show for you and that's when you get trouble. And it was a classic when I was in [Pink Unit] cos that's exactly what happened down there. We had two Mongrel Mob inmates and 
they were both vying for power, trying to raise their heads up, you know. There has always been a kingpin, there always will be, there'll always be a dominant gang group. But getting them to do your work, you're asking for trouble.

- Male, 4-10 years service, Alpha Jail.

The cliques also depended on having some kind of code or informal social control mechanism to prevent members, or non-members, informing on them to management. Although the cliques were limited to around fifteen members each, they exacted an inordinate influence within the entire officer group in relation to the flow of information. The structure for this was already in existence with the traditional prison culture value of 'not narking'. However, as adhering to this could no longer be assumed, the sanctions for its breach needed to become more explicit and menacing at least in the minds of potential informers. The general officer group were convinced that informing on the cliques' activities was tantamount to 'offending against the officer group solidarity'. The cliques then reinforced this informal rule against narking as it suited their purposes. They had been so effective in convincing the general officer group not to do so that they rarely had to 'discipline' indiscrete officers themselves. After all, it was preferable not to offend the cliques, especially the Bros, because in times of unrest in one's own unit, a simple walk through by the Bros could prevent any number of disruptions.

At the lighter end of punishment the offending officer could become the centre of humour. Practical jokes would be played on them, such as hiding their possessions, moving their car and other such 'subtleties' to let them know that their indiscretion had been detected:

It's not like you can really ignore them (indiscrete officer) because you've still got to work with them, but they become the butt of the humour. It's silly really, sometimes we're exceedingly childish. Its lots of petty things, like keeping things (information and physical items) from them, hiding things - actually it sounds really stupid when you say it out loud (laugh).

- Clique member.

Alternatively, and with more serious effect, officers reported the setting of traps to make the offending officer increasingly paranoid until they left the job: 
If an officer has narked or something to senior management, and I've been part of this, we've set little traps. Oh, 'stuff' happens. Like coming on shift and finding doors left unlocked and you've unlocked them to make them look bad, as if they're not doing their job properly. That has happened lots. Its revenge! The staff member became so paranoid about this they transferred to another unit as a result.

- Clique member.

As a result, non-clique staff turned a blind eye to the situation or risked ostracism:

What happens is that you'd know a lot of stuff so you'd just pack it away and get on with it. It doesn't mean to say that I buy into it, cos I don't.... It's like you're just not there, like, you know about things but make sure they're not done right in your face and you just, you know, you just ‘don't know'.... That's why I like being in [Alpha] jail cos you're away from a lot of that shit.

- Female, 4-10 years service, Alpha Jail.

As this extract demonstrates, location choice could insulate one from 'knowing' and thus alleviated any cognitive dissonance that may result. Further, as indicated above, it was easier to employ the traditional officers' script code that if it was not seen it was not their business and they thus did not need to feel in any way obligated to respond. Officers became aware that it was simply easier to get along with the cliques, or to at least remain invisible to them, than be antagonistic to them:

There's one group (Bros) that you're better off getting onside with them than offside with them. If you get offside with them they can make your life misery.

- Male, 4-10 years service, Alpha Jail.

At the same time, many felt that management were unsympathetic when they did raise concerns about clique behaviour, which then reinforced the reluctance to inform:

They've forgotten what it's like to be a staff member and what we have to go through because they're now in a senior management role. It's not that I would cover up but I'm not going to say anything. In the past if we've given information to management it hasn't been acted on the way that it should have and you're still left to work with the staff in your unit and it can make your life hell. It's a catch 22 situation, but basically I'm not going to dig a hole for myself because someone else wants fucking toilet paper for free.

- Male, 4-10 years service, Beta Jail. 
It was evident through the internal investigations which were being carried out at the time of my research that management were aware of the cliques' activities. However, no member of the Bros was under investigation then whereas several members of the Black Mafia were. Officers were also cognisant of this situation and the implicit protection that the Bros were receiving, although understanding the dynamics did not make the pains associated with the existence of the cliques any easier:

\begin{abstract}
It's quite weird isn't it? It's almost like a gang situation. As far as senior management are concerned, as long as they (Lime Unit) are running under budget, not having escapes, not having to answer too many question to the Ombudsman or Ministerial Complaints about treatment to the inmates they're quite happy with the way it is and they won't split that unit up. The inmates know that if they do take on a [Lime Unit] staff member they're going to be answerable to the whole lot - it's like a pack situation, you'd have to be pretty dumb to take them on. So no, they'll never get split up. They (management) want to keep them cos they're useful. Weird isn't it?
\end{abstract}

- Pakeha, 4-10 years service, Beta Jail.

It was likely that management were reluctant to condemn the Bros because, even though they acted through illegitimate means, they were successful at reinforcing control within their unit and across the prison. They were indeed useful to management, whereas the Black Mafia threatened the efficient running and organisation of the prison ${ }^{167}$. Such implicit protection of the former group, however, further weakened the perception of management amongst the general officer body, as it was clear that management were inconsistent and weak in the face of this powerful group. Both traditional and new style prison officers had been alienated in this way. The cliques threatened the values of both and were being condoned by management.

Overall, then, the introduction of unit management had further weakened the traditional prison officer culture and group cohesion. Paradoxically, though, a sense of homogeneity and unity could be achieved through the congregation of like-minded others within the units. As a result of the officers' manipulations of this arrangement, cliques formed, seriously undermining the objectives of the prison authorities.

\footnotetext{
167 As an example, the alleged sexual relationship between a female Black Mafia member and an inmate had caused at least one jealousy inspired physical confrontation between inmates.
} 


\section{Restoring Control}

It was only when the influence of the cliques, instead of being contained within their own units, spilled over into the running of others that management was compelled to act against them. This happened in Alpha Jail. A clique member reported for an early morning shift after spending the entire evening at a prison village party and was still intoxicated. As this was perceived as threatening the safety of the entire officer group and security of the prison, the Yellow Unit officer on duty refused to provide him with keys or a radio and sent him home. A flurry of communications between clique members and those on shift and at the village ensued, some of an allegedly threatening nature. Regardless, the Alpha Jail officer continued to refuse access to the intoxicated officer, as related to me by another officer:

\footnotetext{
There was this one time right, [Bro - ex-prison officer's name] come in drunk and [non-Bro - senior officer's name] told him 'you're not capable of working, go home'. Come on, he was obviously drunk. Any senior officer in his position would have done the same. That guy (non-Bro) got lashed about it. That man (ex-Bro officer) was a danger to himself and to his fellow workers, but he was a Bro, that's supposed to make it alright?

- Male, 10+ years service, Beta Jail.
}

A management investigation ensued, resulting in the dismissal of the intoxicated officer. As a consequence, the Alpha Jail officer was shunned by the clique. However, as he was a respected senior officer there was little that the clique could do without fear of recriminations themselves. At the same time, others realised that the cliques were not 'untouchable'. On this occasion at least, seniority and length of service had been able to outface the power of the cliques. And in this very visible confrontation between the old power base and the new, management had backed the former. To have backed the latter would have been to officially condone their manifest illegalities. It was thus clear to members of the cliques that if they were to survive, their behaviour needed to be withdrawn further into their units. This was to lead to more covert activities and strategies of 'covering up'.

Furthermore, the tolerance of overt misbehaviour continued to diminish as increasing numbers of officers, attracted to the job due to the promises of what the new Head 
Office script was designed to offer them, adopted a work style that was incompatible with clique culture. The high number of new Pakeha recruits also began to outweigh the power of the ethnic cliques. Longer serving staff who had experienced the days of strong clique behaviour, but had been outside the clique groups, were pleased that these were losing favour:

I'm glad we've got more European staff (joining) here because one of the problems of (pause) well some of the cliques were culturally based, which had negative connotations in terms of races. Things like the Black Mafia and stuff, you know, the Bros. It still goes on, it's a bit tongue-in-cheek now, but it has been more ominous.

- Pakeha, 4-10 years service, Alpha Jail.

Ironically, then, the changing (and unintended) demography of the prison officer group made the cliques increasingly marginal and powerless. They also became selfdestructive. For example, during a typical off site prison party, attended by a cross section of the two cliques and other prison staff, a mid-status level Bro challenged the presiding Bro kingpin. A physical fight ensued. As a result, in a hitherto unprecedented action, the Bro kingpin requested a transfer out of Lime Unit. In the months that followed, the influence of the Bro group throughout the prison diminished significantly as the Bros were powerless without the solid structure that had emanated from their kingpin:

\begin{abstract}
Occasionally people decide to get pissed up and decide to be fucking dicks, and that's what happened eh. It can create a real bad situation real quick because you get things that happen on the spot and that can have a major fucking impact on the jail. I remember that bloody do, two staff members got fucking pissed off and had a fucking scrap, fucking huge effect on the jail eh. [Kingpin Bro's name] won't even work with [mid-status Bro's name] so he moved unit, and that's fucking unheard of. You can't replace [Kingpin Bro's name], [Lime Unit] is fucked. \{my emphasis\}
\end{abstract}

- Pakeha, 4-10 years service, Beta Jail.

Meanwhile, because they did not have a role as security enforcers, the Black Mafia was systematically being targeted through internal investigations. Individuals either had their employment terminated or resigned to avoid formal dismissal. From being initially untouchable when my research started, it now seemed no one was beyond 
reproach and the techniques of the past, such as intimidating staff, including management, were losing their effect:

\begin{abstract}
There's the tit for tat thing. There's the 'we don't like what you're doing, but you know too much about what we've been doing'... They're (Black Mafia) one group where those who talk get threatened. And in this environment a lot of people have a lot of skeletons - all the way up to management. Some of these people are very clever, they sit there and go 'continue this on and watch out'. Problem is they don't have any dirt on some of these new fellas (externally appointed managers). \{my emphasis\}
\end{abstract}

- Pakeha, 4-10 years service, Alpha Jail.

In this sense, it seemed that the unit principle had triumphed - the deviant adaptations which had occurred within the traditional arrangements had been stopped and order was returned on a unit-wide basis. Unit management was to be a panacea, the new way forward for the prison service. However, the withdrawal into units, out of the gaze of management, had resulted in the formation of unintended power relations. The resulting cliques had been more deleterious to the objectives of the prison authorities than the problems of the traditional culture that the new way was supposed to replace. The influence of these cliques when they had been at the summit of their power had been to undermine general officer relationships which the clique members had been allowed to get away with by appeals to, or enforcement of, the traditional prison officers' code of silence. By so doing, this had further cemented the division between the general officer body and management, particularly when, on the rare occasion that the silence was broken, management were unresponsive to officers concerns. Notwithstanding their own demise, that the cliques had been able to flourish was a warning about what the destruction of the old style prison officer unity and cohesion had unleashed: variations and reformulations of this culture, making it unpredictable, corruptible and often out of control. 


\section{CHAPTER EIGHT: EACH DAY IS DIFFERENT?}

Nevertheless, unit management and its new roles had become a permanent feature of the prison system and officers had to adjust to this. In the aftermath of restructuring, new recruits were being enticed to the job by the promises of excitement and challenge - each day was going to be different. At the same time, the legacy of traditional prison officer work demanded that this should be predictable and mundane. In these respects, each day could be different as long as it remained the same. In the past it was quite acceptable for nothing in particular to occur from one day to the next. Both inmates and officers preferred this. But as the number of new recruits increased and more officers sought the promise of a rewarding, challenging and exciting career, prison life had become much more unpredictable and uncertain. This chapter will examine five daily strategies of prison work invoked by prison officers to resolve the tension that now existed between the expectation that each day would be different and the intrinsic need for each day to be the same, that combined the promise of excitement and challenge with the tradition of routine and predictability. These involved: dealing with the daily grind; making the tedious fun; making the minutiae count; making the boring exciting; and making the exciting mundane. It demonstrates the dichotomous, contradictory world the prison officers now inhabited. Every day would be the same while they were also able to assert that every day could be different.

\section{Dealing With The Daily Grind}

Prison officers had to reframe those daily aspects of the job which, if left unchecked could eventually affect their fortitude and might lead to burnout. The daily grind included those small facets of the job which most people in other occupations do not have to worry about. For example, when surveyed about the worst aspects of their 
work, the everyday conditions of the prison environment were frequently cited. In Beta Jail such complaints generally focused on the weather and having to walk around in the wind and rain. Within Alpha Jail the most commonly cited offence to sensibilities was the lack of fresh air and foul smell - a combination of poor hygiene, smoke, and poorly ventilated ablutions heightened by the low grade high starch diets:

\begin{abstract}
Oh man, the worst thing here has to be the odour. The smell, my God, if you're not a smoker you might as well be. Oh and the cell searches and strip searches, they smell real bad. In summer it can make you want to vomit eh. Like first thing in the morning, (we) open their (cell) doors and 'phewee, what died?'

- Male, 0-4 years service, Alpha Jail.
\end{abstract}

As has been demonstrated in other respects (see pp. 47-48 \& 205-206), while Goffman (1961) provided a detailed description of the effects of contaminative exposure which contributed to the inmates 'mortification of self', he and others (see for example Cohen \& Taylor, 1972; Johnson \& Toch, 1982; Parisi, 1982), did not consider the effects that debasing tasks (such as strip searches and cell inspections) had on staff. To perform them, officers employed face saving techniques to reduce mutual embarrassment and distaste. These usually took the form of black humour or parody, such as making remarks like 'come on you know you like it' to the inmate while strip searching (when in reality neither enjoyed the task and it offended the sensibilities of both). Such sardonic humour enabled both to distance themselves from genuine embarrassment. Another technique was selective language use, sometimes combined with gestures in a similar manner. This had the effect of both detaching the actors from the task while also demarcating it as an imposition on both parties from a distant body. For example, rather than saying it was time for a strip search an officer would announce 'security check' with their arms open (indicating that it would be a body search). By detaching themselves from these debasing tasks in such ways, both officer and inmate could implicitly acknowledge their embarrassment and distaste while blaming the circumstance on an unknown distant entity (the faceless prison authorities) saving face themselves.

Equally, negative events were frequently reframed as positive bonding experiences. For example, when officers did go through unpleasant situations together, those on 
shift would refer to this as 'friendships forged in fire', a more significant and enduring relationship than any other friendship or even familial associations:

\begin{abstract}
Family and friends can try and be supportive but they haven't been inside the wire, they haven't had these clowns (inmates) mouth off at you. And though it's appreciated that they try and be sympathetic and caring, it's not quite the same as talking to someone who's been in that situation, you know. See its like, touch wood, I haven't had anyone pull an escape on me and I can only try and be supportive of anyone who's had it done to them. But it hasn't happened to me. I don't know that sickening feeling yet. Touch wood I don't find out! But you'll find amongst prison officers, those who have had an inmate do a runner on them, there's a bit of a bond. They know that feeling. They'll always have that. And all the blokes follow through with them, its like friendships forged in fire. \{my emphasis\}
\end{abstract}

- Male, 0-4 years service, Alpha Jail.

As this extract demonstrates, even with the high level of fragmentation that now existed, officers sought a sense of solidarity. The pains of their occupation had not reduced, thus they still required a means to cope with it - a means of aligning with like-situated others. It was a common assertion by the officers in my research, that regardless of what one may think and feel personally regarding other officers, one 'needed' them as even those that were sympathetic to the officers' stigmatised identity_family and their few (if any) remaining external friends—could not truly appreciate their prison experience. This argument that non-prison personnel 'lacked understanding' stands in stark contrast to the depth of understanding of officers' pains expressed by Crawley's (2004) family respondents, but was also demonstrated by Kauffman's (1985) officers. At the same time, however, without the automatic bonds and affinities of the past, these bonds of mutual hardship became a way of transcending the only other dependable bond—one's unit staff-and enabling more alliances and affiliations across the prison.

Another common stressor was dealing with the disillusionment of not fulfilling one's role of habilitating inmates as they returned to prison quickly after release. Newer staff experienced this disillusionment more strongly than experienced staff because they were more likely to have been seduced by the 'case management' aspects that were promoted in the job. As a result, new staff expected to make big differences in the inmates' lives whereas longer serving staff had rationalised that 'small' changes 
reflected a 'big' difference. The contrast in expectations is evident in the following extracts:

I guess I'm a bit disillusioned from when you first sort of start. I think your hands are pretty much tied for what you can actually achieve and do, you know. There's only so much that you can do. The majority of them do it (programmes) to help get them releases instead of helping them to steer them in a different direction (pause). It can be really unsatisfying when you're just here mainly, mainly for security, making sure that these guys don't escape, don't get back out into the community, rather than trying to sort of help them sort of change their ways.

- Female, 0-4 years service, Alpha Jail.

We've had three go from here (released from custody from his unit) in the last six months and two have already been back in the institutions?! You know that no matter what you do in here they go back out and there's nothing out there. As long as that still prevails it will be very hard for inmates not to reoffend, because money's an issue, education, those are the issues. We can't do much about that. It might sound really negative like I've given up on these guys, but once you see the shit eh. I guess I focus on the little things like getting him to be nicer to his Mrs. and that. You can't stop them offending and if you think you can then they'll shit on you by coming back in. So I just try and make little differences in their lives. \{my emphasis\}

- Male, 4-10 years service, Alpha Jail.

Rather than see recidivism as an indication that they had failed in their work, longer serving officers had adopted the technique of denying that this was anything to do with them. Case management, for such officers, was about preparing the inmate for release (board reports and so on) and ensuring that their stay in prison was humane-a simplified and achievable goal—not about reducing reoffending per se. Thus:

Prison officers are here to lock them up and make sure they stay there. Humane and safe containment, that's it... they're (Department of Corrections) all aimed at reforming people or reintegrating them, you know. But people don't reform they conform. And they conform because that's the easiest way to do a lag and get out of here. I'm not wasting my time and energy on something that just isn't going to happen.

- Male, 10+ years service, Alpha Jail.

Some officers, however, became overwhelmed in the process of learning how to respond to the contingencies that the prison threw at them and were generally unable to see these as 'all part of the day's work'. As a result, smaller problems assumed great importance and became the focus of annoyance: 
I spent six hours straight out at the back gate just letting people in. I mean that's not a stressful job, but you couldn't leave. You couldn't get a coffee. You couldn't go to the toilet miles away. It's like 'don't worry about your personal well-being or anything, you should be good, you don't need feeding, you don't need a drink, drop dead in your own time'... it was so boring, it's probably the least stressful job I've ever come across! But they (management) seem to forget about basic needs, wants, you know. I'd love a cup of coffee and I'd also love to go to the toilet! 'Well what do you need relieving for, for God's sake, do that in your own time'.

- Female, 0-4 years service, Alpha Jail.

As this extract demonstrates, and as shown in chapter six, regardless of the contingencies the job threw up, the expectation was still that one would 'handle one's lag'.

Each day, so the recruitment advertising had claimed, would be different. Certainly, while becoming acculturated, each day did have confusion and misunderstandings as one weaved one's way through the varied applications of informal rules and multiplicity of scripts. However, the more familiar one became with the environment, the more it seemed remarkably routine and mundane. What seemed frenetic at the start was actually an extraordinarily undemanding existence (see p. 178). Thus, more experienced officers emphasised the need to put the job and the daily contingencies in perspective:

The main role of the prison officer is that you've got $x$ amount of inmates when you come onto a shift and you've got $\mathrm{x}$ amount of inmates when you go off. You have no more stress when you go off your shift than when you started your shift. And it's, you know, it's all in a day's work.

- Male, 4-10 years service, Beta Jail.

Of equal importance was the realisation that inmates were motivated to maintain harmony rather than create disturbance. Therefore, one did not need to be hyper vigilant at all times nor be overly concerned about control, but could concentrate on the other, albeit less exciting, aspects of the job:

They realise the importance of having harmony amongst themselves, and usually there's a couple of kingpins that will be like 'none of this crap and none of this crap'. And we have things like 'inmates meetings' and all (that) and these are the issues that they deal with eh. Because they come to realise fast that this is going to be their home like for the next three years, and they don't want no crap, they 
just want, you know, (to) do their lag. So it's far easier for us, they take care of it. We really don't need to be worried about that crap.

- Female, 4-10 years service, Alpha Jail.

Nevertheless, negotiating the mundane reality of their world against uncertain possibilities was the perpetual dilemma of prison work. While prison officers sought excitement, anything that threatened to disrupt that routine made them extremely worried. Displaying such emotions, however, did not support the stoic impression that officers sought to foster in their everyday encounters. Therefore, anxiety and concern was projected onto daily 'annoyances' and manifested as frustration or stress. For this reason, they needed strategies to unwind from such incompatible yet co-existing stressors. One was to reframe 'unacceptable' emotions - fear to anger, for example. Thus, unwinding after the Green Unit riot, although it had created a great deal of stress for officers at the time, was not explicitly a release of anxiety but a reframed expression of anger within the group:

\begin{abstract}
All the business with that riot, that was quite stressful. (The) Department (reaction was a) waste of time afterwards. The counselling session - what a joke... what they should have done is let us talk amongst ourselves... but [Upper Manager's name] didn't take any interest in the staff at all. I went to the (de)brief session. They had some shrink in, who's outside the jail anyway so straight away they didn't have any respect. Then the stupid bitch went and said 'oh it's okay to cry if you want to'. No one wanted to cry? She shouldn't have said that... boy, she got ripped to pieces cos that's what we were after. We were so angry and frustrated by the big fuck-up by management.
\end{abstract}

- Male, 0-4 years service, Alpha Jail.

All officers could identify their strategies for unwinding after work so that they could at least try to remove their prison identities when they went home. These included taking a nap or shower after shift; stopping off at the pub; taking the 'long' way home; having a cigarette even though they were a non-smoker; walking the dog; even the process of simply taking off the uniform:

I think our uniform is a good part of the job because when I take my uniform off it's like I've changed my mind. I'm sort of thinking 'it's my life now, what am I keen to do?' Whether it be go for a run or work on the car or do the lawn. Then you put it back on and you switch back to work mode.

- Male, 0-4 years service, Alpha Jail. 
The problem was, though, that they could never actually remove the identity completely. The mortification of self brought about by joining the prison service meant that their prison self had become their new self. As Crawley (2004, p. 249) stated “...the potential for role engulfment is high, and officers' ability to come 'out of role' low... a striking aspect of prison work is the strain of living in, and moving between, two worlds - only one of which is contained within high walls”. Similar to the findings of both Crawley and Kauffman (1985, 1988), the officers' social world had narrowed to the point where the prison encroached on all facets of their life, not just their family. There was nowhere where they could be anything but a prison officer:

\begin{abstract}
Its not that we don't want to know them (outsiders) it's (that) they don't want to know us because it's a dirty job. We're talking about people that society don't want to know. 'Lock them up and throw away the key', that's their attitude. They don't want to know that they are human and really are capable of doing those things. And I look after rapists and murderers and child molesters and granny bashers and they don't want to know that. It's an unpleasant thing in society, they don't want to know. There is a bit of stigma to it, and we wear that. \{my emphasis\}
\end{abstract}

- Male, 0-4 years service, Alpha Jail.

As this extract demonstrates, working with the detritus of human existence was experienced as 'deeply discrediting' and shameful to them, solidifying the gulf that existed between their prison world and the world beyond this (see Goffman, 1968; Walsh, 1975). Working closely with inmates, officers had developed sympathetic identification of them as people rather than human garbage. Officers needed to do this to normalise and make sense of their work, to create a sense of meaning when, to outsiders, there was none.

At the same time, this sense of rejection from society could be used to justify their cultural insularity to themselves - as if it was not them withdrawing from outsiders; instead, they were as much victims of the prison as inmates were. As a result, officers were bound closer to the prison, insulating themselves from at least some effects of their stigmatised identity while at the same time immersing themselves still further in the pains of their occupation. 


\title{
Making Each Day Fun
}

The second strategy invoked by prison officers involved transcending the tedium of the daily routine by making each day fun, trying to ensure that each day would be enjoyable. Fun in prison could take on many guises, such as: gambling ${ }^{168}$ within the officer group or between officers and inmates; bringing in food and making the preparation and consumption of the meal the focus of the shift; looking up the nation's ugliest inmates on the computer system and imagining 'romantic pairs' between various male and female inmates and so on. In such ways, each day then had the potential to be different because of the variety of 'fun' that could be experienced:

\begin{abstract}
Every day is different. I always just go out there to try and enjoy the day because if you don't it makes the day so long eh. It really does, it's no fun at all. You've got to have a laugh. We always used to say, the group of us that started together, 'if we can't laugh in the job you're going to end up crying'. So I'd rather have a good laugh about things. So that's just my approach. I like to have fun every day. \{my emphasis\}
\end{abstract}

- Male, 4-10 years service, Beta Jail.

The anticipation of a fun shift made it possible for officers to look forward to coming to work, whether it was to hear the gossip, make their own contribution to it, or just generally have fun as a team:

If you're not in the chain you're not alive. It can brighten your day to find out
information and have a bit of a laugh. You know, 'who stuffed up yesterday?'
Like [probationary officer's name] locking himself in [inmate's name]'s house
(laugh). Information is really important. You hear all the goss, even if it's not
true (laugh). You know, we sit there and go 'now who should we make up stories
about?' That's frightening isn't it? That's what keeps people alive around this
place. You've got to have a bit of humour every day, you know. You come back
from the weekend 'what's the goss?' I don't want to hear 'well he got this job'. I
want to hear the goss! I look forward to it. You know, (the) slightly seedy side,

${ }^{168}$ Gambling is officially forbidden within the New Zealand prison system. Although it had very little
monetary value (chocolate bars were the most common item to gamble with), it created fun while
passively breaching the prison regulations. According to Burton (1945), gambling was the great
unifying force in the early 1900 s New Zealand prisons. It would seem this tradition had continued.
Officers and inmates would frequently gamble together even though this placed the officer in a
seemingly compromised position of not only possessing guilty knowledge but also being in breach of
the formal rules. Placing bets covered a wide range of activities, it could be on sports teams on the
television or inter-unit games, whether or not someone got parole, what was on the menu for dinner -
the potential occasions were endless. Indeed, the Dominion newspaper (Unauthored, 1997l) reported at
the sentencing of an inmate for an additional ten years he turned to one of his guarding officers and
smiled "[t]hat's a bar of chocolate you owe me" (p. 8). 
who's making an arse of themselves, who's bonking who. The information you can find out, but the real goss - by God it may not be true but it makes you feel better. It could be completely without foundation, but who cares? It's fun. You can spend the rest of the shift talking about it. Who cares who got what job, unless they bonked someone for it - then I'd be interested (laugh).

- Female, 4-10 years service, Alpha Jail.

Fun not only passed time, but, as a secondary adjustment, also enhanced camaraderie, the sense of belonging to a group and raised morale (see also Crawley, 2004). It also gave officers a means with which to reframe boring aspects of the job by making them a game. Officers would thus entertain themselves for hours by making the transference of a boring task to another officer the equivalent of the children's game 'tag you're it', with the loser having to fulfil the unwanted tasks:

I get so bored. I'm shocking sitting in there (guardroom). We got this trick from the [Lime Unit] guys who'll be standing with the phone up and they'll radio and say 'phone call for you'. So you go in there and no sooner than they give you the phone and they're out the door. There's no one on the phone! That's a ploy to get you in so they can get out. It's unbelievable the tricks we play on each other for fun. We all hate being in the guardroom with the phone ringing all the time, or the computer, or the paperwork. I'd much rather be out there with the inmates. Doing what you're employed to do, deal with the inmates. It can go on all shift 'phone call for you' (laugh).

- Male, 4-10 years service, Beta Jail.

Crawley (idem) argued that such pranks were an important part of the officers' world. That engagement in them not only fulfilled the function of amusement for participants and observers, but also functioned to maintain the informal hierarchy of the group. Thus, in the above example, the officer with the lowest informal status would be left to fulfil the boring task. This reinforced that officer's standing while reinforcing the power of his/ her more senior colleagues to 'pass off' such tasks onto their (informal) juniors.

Officers would also entertain themselves, particularly on night watch shifts, acting as pseudo-psychologists assessing inmates files:

You sit down and think 'what would make you do something like that?' 'Why did you rape that eighty year old lady?' You know, 'what gratification did you get out of that? What did you gain out of that?' I just sit there and look at them or their files and try to think what would be going through a guy's mind to do something like that. It's funny though, if makes the day go faster.

- Male, 4-10 yeas service, Beta Jail. 
As this extract demonstrates, officers actively sought to understand inmates and their offending behaviour. Such 'understanding' contributed to officers' development of sympathetic identification with their charges, masking their 'human garbage' status and humanising them (see Goffman, 1968). At the same time, the officer might glean a 'new' piece of information about the inmate, and if injected within the officer group appropriately, might elevate them in the information hierarchy.

Having fun also had the added benefit of negotiating control while making both the officers' and inmates' lags more bearable. This was achieved through an economic and social exchange - officers provided certain luxuries to inmates in return for cooperative control in the unit. At the same time, these treats were mutually enjoyable and fun for the officers:

\begin{abstract}
There's a hell of a lot of goodwill (from officers). I think if that goodwill was withdrawn if wouldn't be a fun place to be, not for inmates, and not for us. Inmates would get virtually nothing and we'd all be bored out of our brains. Like, our inmates have a video evening about once a month, and that means that I, or somebody else, does the shopping for them at Pak'n'Save (supermarket) and buys the pies and so on. They wouldn't have that or they'd have to buy it through bloody P119s, which would cost them a bloody arm and a leg. They wouldn't have their video nights. They'd have to put up with the crap videos that they'd (management) get. Oh there's all sorts of things that they wouldn't have, you know, lots of things that just wouldn't happen if it wasn't (for) the goodwill of officers. But we enjoy it. It can be a real kick organising these things, and we get to enjoy the evenings too. We're stuck here just like they are so we might as well have some fun.
\end{abstract}

- Male, 0-4 years service, Beta Jail.

Overall, then, strategies for making each day fun not only ensured their job was enjoyable, but also 'mercifully killed time' (see ibid, 1961, p. 67), enhanced collegiality and lessened the pains of their occupation. In addition, the above cooperative exchange with inmates also made the officers' lag a little easier. In such ways, officers could assert that each day was different even though the routines and daily tasks were, in fact, the same. 


\title{
Making Each Day Count
}

Officers also tried to ensure that their at times mundane existence could mean something - that each day would count. There was a feeling that while they had been employed for security or, for newer officers, case management, they were now overly consumed by administrative tasks, devoid of intrinsic purpose, such as 'paperwork' Therefore, attaining a sense of accomplishment could be difficult:

\begin{abstract}
When I started we were here strictly to walk the floors and make sure everything is okay. Now we've got to do triplicate paperwork almost, and then with the computers coming on deck (laugh). I started here as a prison officer just to walk the floor and be here. But now we've got to do case management and virtually run around for them. I think we're glorified babysitters really. That's where it's gone from, from doing custody and security work, to being highly paid babysitters.
\end{abstract}

- Female, 4-10 years service, Beta Jail.

However, making each day count did not actually require anything dramatic to happen. The absence of activity or trouble could be framed as a meaningful achievement (see also Crawley, 2004; Liebling \& Price, 2001):

\begin{abstract}
Every day's a good day to me. It's having gone off (shift) when there could have been a potential bloody gang war in the unit. The fact that, you know 'well I did something really worthwhile today, we could have had a blood bath in the unit and things will go nicely tomorrow'.
\end{abstract}

- Female, 4-10 years service, Beta Jail.

Even disruptive events could be reconstructed as having some productive purpose. For example, the Green Unit riot was reframed as unifying the officer group and highlighting the perceived ineffectiveness of management:

It makes me laugh, I feel cynical about it. It had management ducking for cover, justifying everything. Frankly, anything that puts a bit of pressure or fires a shot up their backsides is a good thing. As long as positive things come out of it it's par for the course.

- Male, 4-10 years service, Beta Jail. 
In the reframing that took place, the riot gave staff the confidence that they could handle anything and a renewed sense of purpose within the job:

\begin{abstract}
The riot that we had here, I was glad to be part of it and to have come through it afterwards. The experience and the fact that I was able to handle it and work through it and support the fellow staff that were there. I felt that sort of helped me, you know. Feeling like I could still function, rather than feeling like I had to function because I need this job, I need the money... there's nothing like the smell of fire and missiles whistling round your ears that will galvanise your attitude, that's for sure.
\end{abstract}

- Male, 4-10 years service, Beta Jail.

Crawley (2004) similarly identified riots as a mechanism to bolster staff solidarity and re-establish purpose. Notwithstanding this, an important difference between Crawley's officers and those in this research was that for her, riots also functioned to polarise officers and inmates, re-establishing what may have been a troublesome relationship line. Although the officers in my research referred to themselves triumphantly as 'the Green Machine’ (see p. 261), which might suggest something akin to what Crawley found, it was in fact a reference to their rekindled (albeit temporary) unity and power. Furthermore, the polarity here was between officers and management. The former perceived the latter as incompetent, disorganised and lacking faith in the ability of their staff to do their job. On this basis it would appear that the antagonisms between New Zealand prison officers and management in the aftermath of major restructuring were more acute than those experienced in England.

Officers could also combine 'making each day fun' with 'making each day count'. For example, with the shortened social distance between officers and inmates it had become not uncommon for officers to organise special events for inmates to mark various occasions such as twenty-first birthdays or parole release for long serving prisoners. Officers were able to make the inmate's lag more bearable, secure generalised compliance (other inmates would behave as they wanted their significant dates marked as well), while having fun and providing a sense of achievement and purpose for themselves:

Oh you should have seen him down at his twenty-first. We put on a big party for him and he was almost crying. Heaps of food and that. And he said 'what's that for?' And I said 'I told you we were going to give you a party' and he said 'you're joking aren't you?' And he opens these cakes and everything. He got all 
teary eyed.... He's a good kid. Hopefully he'll go on the right track when he gets out. Its days like that that make you feel like you're really achieving something here.

- Male, 4-10 years service, Beta Jail.

While many officers were sceptical about making each day count through participation in the programmes that were offered in the prison, the exception to this was Straight Thinking. This was a seventy hour cognitive skills programme involving a collaborative effort between a small group of inmates and one or two officers using basic cognitive reframing techniques to address the inmate's "lack of critical reasoning required to live effectively in society" (Department of Corrections, 2004d, p. 6). As they had daily contact with inmates, officers felt they were the 'experts' on inmate behaviour and had a better appreciation of what inmates needed to at least temporarily delay returning to prison. Accordingly, many officers would go to extra lengths to provide instruction for inmates. Goffman (1961) argued that this type of behaviour was a secondary adjustment that reflected an over commitment to the organisation. However, the officers in this research would vehemently dispute that their behaviour was motivated by the organisation. Rather, they would claim it was their sympathetic identification with the inmates and desire to 'achieve something' that compelled them. Indeed, there was no formal recognition for such efforts so it is doubtful it was a reflection of commitment to the organisation; the motivation for officers was simply to make each day count through their involvement:

\begin{abstract}
Classic example is [prison officer's name]. He's very much into this Maori culture and he had a group of guys (inmates) down in [Lime Unit] and a few used to come up from [Grey Unit] for his cultural classes. It was awesome. He gave these guys self esteem and self worth and self discipline. You know [prison officer's name] did that in his own time. No one (management) ever thanked him for that. You could see a real change in some of the guys and good luck to him if it works. But no, it doesn't get recognised, doesn't get treated nicely. But if you cock-up - boy does that get recognised fast.
\end{abstract}

- Male, 0-4 years service, Alpha Jail.

As this extract demonstrates, although officers could see value in such behaviour, both for themselves and inmates, there was a sense that management did not appreciate their efforts which again contributed to their cynicism and resentment towards them and general feeling of being devalued (see also Crawley, 2004). 
Ironically, given their initial reaction to case management, this had also come to have a positive meaning for many officers. They were able to achieve something; they could be more than just turnkeys:

\begin{abstract}
My workload's become more diverse. I'm finding I'm filling out my hours and I still don't have a lot of time. Whereas in the first few years here it was a doddle. The expectations on staff weren't that high, we were just turnkeys. The mission statement had nothing to do with reducing reoffending; it was just humane and safe containment and custody. Case management was just starting to creep in, which we all laughed at, now you can see it's fully on line and we have to embrace it. Before then the job was not demanding at all. Don't go telling anyone, but I actually enjoy case management now.
\end{abstract}

- Male, 4-10 years service, Alpha Jail.

As such, case management enabled many staff to take pride in their work where previously there had been none:

\begin{abstract}
In hindsight I'd have to say I prefer the way we do it now. Under the old way I was earning good money for comparatively little. I say 'comparatively' because other people (outsiders) were working fairly stringently for a lot less income... you could sit back and do the crossword, but at the same time, at the back of my mind 'why am I here?' You felt a question of guilt and a little bit of shame creeping in. Like sometimes, I mean it was almost a competition to see who had the cushiest ride. You'd look at the roster and think 'sweet I'm on this shift so there won't be much work'. But you did begin to feel a bit ashamed.

- Male, 4-10 years service, Alpha Jail.
\end{abstract}

In this way, a small part of the stigma and shame associated with prison work had been alleviated. It was still 'dirty' work, but at least they could engender a sense of meaningfulness from it - they could make a difference.

Interestingly, 'you can make a difference', a phrase used in the advertising campaigns for new officers, had become a part of the prison officers' own scripts, both old and new. Security had become a taken for granted aspect of the job, an assumed achievement. In contrast, this new aspect of the Head Office script was what was now making their existence count:

There was this guy, a murderer [name and details of case given]. He came down here (Beta Jail) and was sweating like a pig. He was frightened to death because he hadn't been outdoors for such a length of time. I said to him 'listen, you know me (from a previous prison sentence), I'll look after you down here, all right, I'm 
going to ask for you to be my case inmate'. I had him for two and a half years. We worked hard getting him into a position of a normal human being. Seriously, this guy was accepting my advice and everything I could offer him... anyway, I went to the (parole) board with him, I made out all the board reports, and he got out! When he got it he put his arms out and said 'thank you Mr.[officer's surname]'. You know, I thought 'what a feeling'. Honestly, what a feeling... I mean that was my most satisfying moment... when I drive home sometimes, it's 'have I done anything useful today?' not 'have I done my job?' Seriously, I'm not bullshitting. 'Did I boost somebody's morale? Point them in the right direction? Stop them from committing suicide?'.... There's been many incidents like that and they really stick with me... the bottom line is do your job so you can say 'I'm happy with that, I made a difference'. \{my emphasis\}

- Male, 10+ years service, Beta Jail.

Even so, there were occasions when an officer would identify a particular inmate and put considerable effort into his case in the hope of achieving some more substantial change. If selected well, the inmate may succeed, providing the officer with a success story and a sense of achievement:

I do personally hold hope for some guys. Over time you get to know some of the inmates inside out, and to some extent you, you know, you have ambitions for them. I think 'once he gets out this time, if we can keep him off the dope we might not ever see him again'. I actually have my fingers crossed that he might be rehabilitated. He's laid down plans to me with what he wants to do with his life, and you know, in a 'going straight' 169 environment. You know he's quite capable, he's a talented guy, if I can help him get to where he wants to go I feel good about that. It's very easy to be very negative about all these guys all the time, that's no sweat. But then some fella comes along and you do hold out hope. You gotta try, you can't turn you back on them. You can make a difference. \{my emphasis\}

- Male, 0-4 years service, Alpha Jail.

If, however, the inmate was unsuccessful, the officer's sense of failure intensified and was experienced as a serious assault of their self worth. The decreased social distance, increased sympathetic identification and invested energy then meant that such officers were likely to blame themselves for subsequent relapses (see Goffman, 1961). It was not uncommon for an officer in this situation to become withdrawn for a time as neither the old nor new scripts prepared them for such 'disappointments'. According to the traditional officers' script, there was no hope anyway for the inmates they were working with. According to the new Head Office script, one was not doing one's job satisfactorily - helping inmates to avoid returning to prison. In this way, new

169 'Going Straight' was the original name for the 'Straight Thinking' programme. Many officers maintained this original name in common parlance. 
uncertainties were developing for which officers were neither trained nor equipped to deal with.

\section{Making Each Day Exciting}

Making each day exciting was based around the anticipation of trouble, in this way each day could be different even if the actuality of such events was to be avoided at all costs:

Well to be honest the job is cruisy (pause). I suppose the unpredictable side of it's good. I'm quite into the old danger side of things. The majority of the time nothing ever happens here, but when it does it's usually quite interesting. It's like you can ask someone (inmate) a question one day and you get a reaction, and the next day you'll ask the same question and get a totally different one. It's never the same. It's the unpredictable nature of this job eh, every day is different. If it wasn't for that it would be a pretty boring job. \{my emphasis\}

- Male, 4-10 years service, Beta Jail.

As with making each day count, the lack of negative incidents could be interpreted as a result of the officers' skilful mastery in manipulating situations and circumstances and out manoeuvring inmates (which contributed to the sense of excitement):

It's a mind game. At any time they know they can over power us. So we have to show we can keep control and it's a mind game really. I've been caught out a few times - 'Mister you've got no keys, we could take you out right now and no one would know', you know. It's quite daunting, you have to meet their bluff and that's the mind games. That's all we're playing all day, cat and mouse. That's all we're playing in a real sort of scenario, you know. Usually we're two moves ahead, but it does at times daunt me.

- Male, 4-10 years service, Alpha Jail.

Another common strategy employed by officers to create excitement was the telling of 'war stories' (see also Owen, 1988; Schwartzman, 1993):

I've got all sorts of bullshit stories but most of them are irrelevant and you'll hear a dozen-a-day stories here. A lot of us are prone to make life a bit more exciting with our stories (laugh).

- Male, 4-10 years service, Beta jail. 
In the retelling, control was never lost, the drama was more pronounced, the violence by officers was embellished and officers performed at a higher level than actual observation would suggest. For example, the Green Unit riot was 'never really that bad' (control was never lost; indeed within six months it was commonly referred to as a 'multiple-inmate disturbance' rather than a riot) and regardless of the incident, officers always 'got the better' of inmates:

\begin{abstract}
I don't think you ever forget the fights you get involved in .... I remember I looked up the wing and there was a fight going on, Black Power and Mongrel Mob going for it... we wrestled them (inmates X \& Y) apart. We took him (inmate $\mathrm{x}$ ) to the holding cell, and we thought 'we've got to go back there', just something triggered. So we threw him into the cell and we ran back. Just as we got back [inmate y's name] and this other Black Power guy started going for it in the middle of the wing. [Officer's name] and I were just into him, on the ground giving him a fair whopping. And it was funny, we had him face down on the concrete floor, and I was on top of him and we were trying to get his arms behind him and [another officer's name] come flying in from the side to really put the boot in, and he looks up at me and says 'fuck this is good fun eh mate'... I've never forgotten it, still laugh about it now (laugh).
\end{abstract}

- Male, 4-10 years service, Beta Jail.

When officers did engage in violence with inmates, their description of it was extremely scripted. If it was culturally sanctioned (according to the traditional script), then there would be some detail in the description, such as 'I cracked him'. If it was more violence than the officers' code of just deserts permitted, the description would be without detail and coded, such as 'I counselled him'. Detail, such as given in this extract, is an indication that the violence was likely to have been of a culturally acceptable level (or lower) but had been embellished for the purposes of the story telling (as it seems excessive, when, if it had been, there would be no detail at all). Notwithstanding this, the embellishment and imagery of these war stories created a legacy of excitement - today might not be different, but tomorrow might be as exciting as this story.

\title{
Making Each Day Mundane
}

At the same time, however, when particularly dramatic incidents did occur, these were likely to be reduced so that each day could continue to be experienced as mundane. 
When events occurred that jeopardised the harmonious balance of the unit, jail or prison, they required reframing to reduce the exciting into a more bearable, controllable aspect of prison life. For example, in reference to the tumultuous events of 1997 (riots, hostage takings and so on, see p.134):

Oh it hasn't been that bad. The riot was just a national standard introduction thing. They were gonna get locked down nine-to-five seven days a week, they got a bit upset about that. Most of those guys got shipped up to Pare (maximum security prison) and then got involved in that riot up there which was pretty much the same reasoning. The Christchurch one was just a few inmates that were trying to make a point about their own individual cases and just seeking attention like that. It's really not as dramatic as the papers make out.

- Male, 0-4 years service, Alpha Jail.

Alternatively, while accepting that exciting incidents involving inmate conflict could occur, many had rationalised that the relationship they held with their unit's inmates would prevent such an episode occurring during their shift:

In a planned situation they'll wait till the shifts with the officers they don't actually get on with, you know. It doesn't look good for them if I have to take them to the pound whereas they don't mind if someone else does it. They know if they're going to bash some (other) inmate on my shift then whatever relationship we had, you know, friendship, it will be ruined. Nah, they won't do it on certain shifts, not on my shift! They actually consider the consequences of playing up on an officer. They might not be that educated and that, but they're not stupid (laugh).

- Male, 0-4 years service, Beta Jail.

One way of coping with this was by acknowledging the severity of a problem, but reducing its effects on them by redirecting its cause. Thus, it was not a breach of security or even a momentary loss of control, instead, it was due to something out of their control - incompetent managers, once again, for example:

It's like with the riot here, management fucked everything up. The staff in the unit had it pretty well sorted. They (inmates) were calming down. And then you get the police with guns circling all over the place because the managers had let them in. I mean, come on, there's no faith in your staff. They don't know (that) what they're doing inflamed the situation because most of the staff were dealing with it fine. Yeah, they were having a hooley, but they were calming down. They were just pissed (intoxicated). We'd contained it, no worries. But oh no, the managers go and call the police. These guys, they've got no time for police. As soon as they see that uniform - that's it, we're off! I mean that's what they're 
like when they're picked up to go to court, they're fine with us until the police arrive.

- Female, 0-4 years service, Alpha Jail.

As highlighted in this extract, with the increased division between officers and management, and their generalised animosity towards them within the new working arrangements, as 'faceless' entities they were frequently the brunt of any perceived inadequacies of the prison system and the target of officers' (and inmates') anger.

The importance of the 'absence of trouble' was reflected in many of the officers' tasks or daily rituals that were focused on achieving this. For example, one of the routine tasks was checking the progress of the wingmen ${ }^{170}$. However, this generally amounted to counting three men where they were supposed to be rather than actually being concerned about the 'progress' of the inmates' tasks or ascertaining whether the activity was meaningful to them. One would not hear 'how was your day?' or 'do you feel you have achieved something?' to a returning work gang. Rather, it was a count of 'six went out, six came back'.

Equally, when dealing with inmates who had committed particularly serious crimes, officers would simply limit their emotional involvement with them. As in the following example, day-to-day pleasantries could be exchanged with them, no matter what they had done, as a way of getting through the day without any draining emotional release:

You get desensitised. You come to work, you treat inmates like you just treat everybody else I suppose, you know, be nice to them. You strike up really good rapports with them. Sometimes you get lost in this world. You actually forget that you're dealing with some horrific people who have committed horrific crimes because you're not dealing with the crime; you're only dealing with that person. $\{$ my emphasis $\}$

$$
\text { - Female, 4-10 years service, Alpha Jail. }
$$

${ }^{170}$ Wingmen are those inmates left unlocked during the day within the wing to perform cleaning duties. Also referred to as 'messmen'. 


\section{Each Day Is Different?}

Once one got used to being in prison, prison work appeared to be an extremely boring and tedious occupation within a drab and depressing environment. The potential for a productive and interesting working life, that each day could be different, could seem minimal. However, as illustrated in Table $8.1^{171}$, that the job looked interesting and exciting were the most significant motivators for many officers in New Zealand to join the prison service.

\section{Table 8.1}

Motivations for Becoming a Prison Officer from Officers at the Target Institution

\begin{tabular}{|c|c|c|}
\hline $\begin{array}{l}\text { Motivation For Becoming } \\
\text { a Prison Officer }\end{array}$ & $\begin{array}{r}\text { Prison Office } \\
\text { Designated Re } \\
(\mathrm{N}=\end{array}$ & $\begin{array}{l}\text { s Selecting the } \\
\text { sson for Joining } \\
\text { 115) }\end{array}$ \\
\hline "The job looked interesting” & $(66)$ & $57.4 \%$ \\
\hline “The challenge/ excitement” & $(60)$ & $52.2 \%$ \\
\hline "For the money" & $(46)$ & $40.0 \%$ \\
\hline “Just needed a job” & $(31)$ & $27.0 \%$ \\
\hline “To help reduce re-offending” & (24) & $20.9 \%$ \\
\hline “Friends/ relatives were prison officers" & $(22)$ & $19.1 \%$ \\
\hline “Always wanted to be a prison officer” & (8) & $7.0 \%$ \\
\hline Job security & (6) & $5.2 \%$ \\
\hline “Other” & (6) & $5.2 \%$ \\
\hline Enjoy working with people & (4) & $3.5 \%$ \\
\hline "Power" & $(2)$ & $1.7 \%$ \\
\hline
\end{tabular}

Note. Multiple responses permitted therefore totals do not equal 100\%. Dotted line indicates the division for Expected N, categories above have positive residuals, categories below have negative residuals.

\footnotetext{
${ }^{171}$ The qualitative data for motivations for joining the job were derived from the questionnaire. Questionnaire returnees were requested to indicate which of the motivations best matched their reason for becoming a prison officer. The motivations were listed as forced response categories, derived from rationales provided within the existing literature to test their relevance to the modern prison officer and the New Zealand context; and concepts from the Department of Corrections advertising campaigns. See Appendix $C$ to view the nature and context of the specific questionnaire question. The two categories of 'Job security' and 'enjoy working with people' were added as categories arising from 'Other' responses. 'Other' responses that were directly related to the nine existing or two additional categories were removed from the 'Other' category and placed in the appropriate response category.
} 
What was of particular interest in the cited motivations for becoming a prison officer was that the first two categories indicated a direct alignment of motivations with the advertised features of the job outlined in chapter one. Although this may seem unsurprising given that the recruitment campaigns used these phrases to attract new recruits, these two features were motivators regardless of which demographic category the officer was in (see Appendix O). This would suggest that, perhaps with 'selective recall', these features had been written into all officers' scripts. The third and fourth motivators indicated the perfunctory or pragmatic appeal of the prison service to this predominantly semi to unskilled workforce and is in alignment with the motivations discussed in the international research literature ${ }^{172}$. One major aspect of the job historically, that of job security, scored significantly low as a motivator. This might be related to New Zealand's low rate of unemployment from the mid-1990s; and it is also likely to reflect the way in which the general restructuring of new Zealand society around 1990 had led to recognition that job security was a thing of the past, belonged to a different era (see advertisement p. 21).

Just as the concepts of the advertising campaigns were reflected within the officers' motivations for joining the service, these also featured highly when officers were asked what was best about the job, as shown in Table $8.2^{173}$.

\footnotetext{
172 The most frequently cited motivators for joining the prison service from the international literature were: money and job security (Jurik \& Halemba, 1984; Kauffman, 1985; Lombardo, 1981; Morris \& Morris, 1963; Myers, 1995); just needed a job (Jacobs, 1978; Jacobs \& Retsky, 1975); friends and relatives were prison officers (Kauffman; Myers); and philanthropic ideals of wanting to help reduce reoffending (Jurik \& Halemba; Kauffman).

${ }^{173}$ The qualitative data for the best and worst features of the job were derived from the questionnaire.
} 
Each Day is Different: Prison Officers and Their Work

Table 8.2

Features Listed by Prison Officers at the Target Institution as the Best Features of the Job

\begin{tabular}{lr}
\multicolumn{1}{c}{$\begin{array}{c}\text { Features Listed by Prison Officers as The } \\
\text { BEST Features of The Job }\end{array}$} & $\begin{array}{c}\text { Prison Officers } \\
\text { (N= 115) }\end{array}$ \\
\hline Workmates as friends/ comradeship & $(51) \quad 44.3 \%$ \\
Challenging/ interesting/ variety & $(51) 44.3 \%$ \\
Meeting diverse groups of people & $(45) 39.1 \%$ \\
Making a positive change to inmates' lives & $(45) 39.1 \%$ \\
The money & $(36) 31.3 \%$ \\
Shift-work & $(34) 29.6 \%$ \\
Holidays - lots of them comparatively & $(28) 24.3 \%$ \\
\hline Responsibility & $(14) 12.2 \%$ \\
Job security & $(11) \quad 9.6 \%$ \\
Skill development & $(9) 7.8 \%$ \\
More time with family comparatively & $(5) 4.3 \%$ \\
Satisfaction & $(4) 3.5 \%$ \\
Meeting [romantic] partner & $(3) 2.6 \%$ \\
Home-time & (3) $2.6 \%$ \\
\hline
\end{tabular}

Note. Multiple responses permitted therefore totals do not equal 100\%. Dotted line indicates the division for Expected $\mathrm{N}$, categories above have positive residuals, categories below have negative residuals.

Similar to the findings of Crawley (2004) and Liebling and Price (2001), collegiality ranked amongst the top two best features of the job. This highlights the value of solidarity within the officer group, even if this was fragmenting. Indeed, when asked what they would miss most about the job if they were to leave, without exception interviewees responded with 'the people':

I s'pose I'd miss working in the team environment eh, with the crew, with the people that you're working with. When you get used to working with people, they're sort of like family really. That's about it, that's all I'd miss, the people. \{my emphasis\}

- Female, 0-4 years service, Alpha Jail.

As demonstrated in this extract, even though they were no longer connected to the prison wide group, the value of the 'prison family' persisted. Their job was still 'dirty' and extreme and their identity was as stigmatised as ever. As such, connections with 
the outside world continue to be cut off. Therefore, an important feature of their life remained bonding with like-situated others as a coping strategy. In these respects, solidarity and identification at the unit level had intensified, notwithstanding the shortcomings and unintended consequences of unit management that have been demonstrated in this thesis. Obviously, people relationships remained important features of prison officer work. However, joining the service because of a specific interest in such work was not a strong factor as only 3.5\% of the respondents in Table 8.1 gave 'enjoy working with other people' as their reason for joining the service. It was upon joining that the value of people and relationships became important as they learnt to cope with their new stigmatised identity and were increasingly cut off from the outside world (see Goffman, 1968).

As has been demonstrated throughout this thesis, and as Table 8.3 confirms, the new management structure was the greatest source of annoyance for officers.

Table 8.3

Features Listed by Prison Officers at the Target Institution as the Worst Features of the $\mathrm{Job}$

\begin{tabular}{|c|c|c|}
\hline $\begin{array}{c}\text { Features Listed by Prison Officers as The } \\
\text { WORST Features of The Job }\end{array}$ & \multicolumn{2}{|c|}{ Prison Officers $(\mathrm{N}=115)$} \\
\hline Changes (explicit) & (89) & $77.4 \%$ \\
\hline Managers & (71) & $61.7 \%$ \\
\hline Conditions & $(66)$ & $57.4 \%$ \\
\hline Other Prison Officers & (39) & $33.9 \%$ \\
\hline Other & (39) & $33.9 \%$ \\
\hline Inmates & (26) & $22.6 \%$ \\
\hline Assault/ threat of & (13) & $11.3 \%$ \\
\hline
\end{tabular}

Note. Multiple responses permitted therefore totals do not equal 100\%. Dotted line indicates the division for Expected N, categories above have positive residuals, categories below have negative residuals.

The distance between officers and management, particularly between officers and Head Office, created discernible levels of stress and resentment amongst the officer group. Officers did not express this as a macho assertion of dominance or expertise within the prison, but rather as a reaction to the frustration of conflicting orders seemingly created by people who were detached from their operationalisation. Indeed, 
'management' was the universal feature that officers would not miss if they were to leave the service:

I wouldn't miss the management. I wouldn't miss them at all. I wouldn't miss their protocol that they're trying to introduce, those damn memos that they keep sending out, the paperwork, far out! Yeah, it's the management. Fuck I wouldn't miss them. This would be the perfect job if we got rid of half of them.

- Female, 4-10 years service, Alpha Jail.

It was as if management had become so distant that they were now experienced dayto-day as 'memos' rather than as 'people' and it seemed that their only interest in officers was the paperwork that they produced, contributing even more to the officers' cynicism. For the officers, the increased bureaucracy prevented them from doing the job which they felt they were employed for and which should be centred around security and also (with case management) reducing reoffending:

\begin{abstract}
Even in the five years that I've been here, the paperwork that's increased is just huge. And like the computers and that. I mean that's the way things seem to be going. But for us on the floor, it just makes things three or four times more time consuming. Whereas they're forever sending us out directives that we have to be in the compound with the crims at all times. How are we supposed to do that? To do a muster now you've got to go round and name-to-face everybody, you've got to come in (to the guardroom), you've got to write it up in the book, you've got to log it into the computer which takes three or four minutes if it's on screen already, and then you've got to go through and check all sixty (inmates) on the computer and move those that haven't been moved. You'd spend about half an hour in here doing your paperwork when you should be out there. There's just not enough hours in the day to do it properly. It's changed so much. Whereas before you'd be out there, you'd be in (the guardroom) for five minutes, grab your muster (board) and do your muster, come in, log your muster in the book, five minutes you're all over and back doing what you're suppose to be doing.

- Male, 4-10 years service, Beta Jail.
\end{abstract}

What was particularly problematic about the increased bureaucracy and paperwork was that rationalisation - and thereby less bureaucracy and paperwork - was one of the 'promises' that had been used by Head Office when trying to restructure the prison service (see p. 36). This, though, had failed to eventuate. Indeed, the number of required forms for any single activity had increased and computerisation added a further layer of recording on top of the paperwork (rather than replacing it). In these respects, it was as if those responsible for restructuring had taken a great deal away from the prison service while giving very little in return. 
Such worries and concerns about management and the pace of change are probably predictable. What was not so predictable, at least according to most prison officer research (see for example Kauffman, 1985, 1988), was that 33.9\% of them listed 'other prison officers' as being one of the worst features of the job:

\begin{abstract}
There's too much personality clashes now. It didn't used to be like that. The backstabbing and the gossip and the in-house bullshit, that's the only thing I ever found frustrating in this job. It would make life a hell of a lot easier if we stopped fighting amongst ourselves. Don't get me wrong, some of the guys here are the best guys you'll ever meet but there's a lot of shit-heads too.
\end{abstract}

- Male, 4-10 years service, Alpha Jail.

This, particularly when taken into account with the very low value of 'working with people' in Table 8.1, confirms to us the uniquely damaging effects of restructuring to the New Zealand prison service. The associations of the past were fragmenting, each day was indeed beginning to be different, but without the loyalties, cohesion and unity that officers had previously been able to rely on to see them through any difficulties the job threw up. Without these qualities to guide and protect them, the very features of the job that was held out by Head Office as one of its biggest attractions, that it was going to be more exciting and dynamic, were becoming an alarming reality for them and caused great consternation within the group:

\begin{abstract}
You know what I hate? Constant change in the department, and I mean constant change! Since we became a regional prison this place has changed every day. There's something changed in here of every day of every week of every bloody month for the past eight or nine years! It's constantly changing. A lot of it is just change for change's sake. You can't run a place when things are forever changing like that. You can't get yourself focused, you know. There's always change, there's always someone wanting to change it for some reason or other.
\end{abstract}

- Male, 10+ years service, Beta Jail.

When confronted by such significant challenges, it would seem logical that a group would attempt to re-establish the status quo which they were familiar with. However, with the newly created prison structure, this necessitated 'circling the wagons' (see pp. 264-265; also Crawley, 2004) at the unit level, rather than across the prison. Officers still needed to feel pride and meaning, which they now largely achieved through their relationships at this level of prison existence. This was where power in the prison now lay. Here, they could invoke their various strategies that subverted the prison 
authorities' intentions of creating a challenging job where each day would be different, by ensuring that it remained routine and predictable. In this way:

\footnotetext{
Everyday could be different, every day could be the same. It all depends on the day. If it's a good shift you have a coffee and a laugh about it and go home. If it's a bad shift you have a coffee and laugh about it and go home. \{my emphasis\}

- Male, 0-4 years service, Beta Jail.
}

\section{Conclusion}

The central task of this thesis has been to explore the day-to-day experiences of prison officers in a typical New Zealand prison as they attempted to make sense of their work in the aftermath of unprecedented organisational change. This massive restructuring process began in 1991 with the formal intention of aligning the prison service with the newly reorganised New Zealand state sector and public service in the wake of major society wide economic restructuring in the 1980s. The prison authorities envisaged a complete reorganisation of the prison officers' job from its mundane security dominated tasks to a more challenging and demanding role incorporating unit and case management - each day in prison was going to be different from now on. Coinciding with this, the career structure was flattened, quasi-military insignia formally removed and a nationally recognised qualifications structure initiated. In addition, the demographic structure of the prison service was intended to be radically altered with the recruitment of women and more ethnic minority officers. In such ways, it was expected that a new, dynamic ésprit de corps would then emerge, eradicating the traditional prison officer culture which Head Office perceived as the feature that had prevented the prison service moving forward. It was thought that destroying the officers' occupational culture would place power firmly in the hands of management and enable the emergence of a new breed of professional prison staff: a staff that would be effective and efficient, embracing the 'private sector attitude' of reward through hard work and achievement. Staff that did not fit this model, it was hoped, would leave the service. Indeed, the reorganisation was intended to be the panacea for all prison service problems. 
Notwithstanding these intentions, as demonstrated in this thesis, this was not what occurred. The 'underlife' of the prison that the authorities sought to remove had become deeply embedded and prescribed in the working routines of prison officers over a hundred years of strengthening homogeneity and unity within the officer body. The result of the revolutionary changes on the prison service then meant that this culture, with its longstanding history and institutional memory was not destroyed but, instead, was destabilised, leading to new, unpredictable and unanticipated patterns in the working life of prison officers.

The occupational culture of the traditional officer group, which Head Office had so vehemently opposed as it challenged their authority, had weakened. The cohesion and unity that had provided a sense of solidarity, meaning and attachment to something larger than them had fragmented for officers. Each day was indeed becoming different, although this was not experienced as dynamic and exciting as anticipated by the prison authorities. They had failed to recognise the importance of stability and routine for the prison. Both officers and inmates required this. Changing one culture did not change the culture of the prison. As demonstrated in this thesis, these new arrangements did not transpire into a workforce of enthusiastic, professional officers, eagerly competing with each other to get the few promotional rewards that remained as intended. Instead, fragmentation, uncertainty, disillusionment and corruption abounded. Most became resigned to the fact that they now had very little by way of career prospects. Longevity in the service no longer counted for much and there were too few promotional opportunities. As a result, officers became more perfunctory in their job and attachment to the organisation, gaining what little meaning they could from the job through secondary adjustments and adaptation of the case management objectives.

As illustrated in chapter five, the injection of a Head Office script on top of the traditional officers' script, and varied acceptance and rejection of either by the now diverse demographic group, had led to a multitude of available scripts. It seemed nothing was clear or prescribed, yet, at the same time, the traditional script still governed many of the informal rules. Learning how to 'do' the job and 'be' a prison officer thus became an increasingly difficult task. This was compounded by the lack of, or unwillingness by, longer serving officers to assist in the induction process. As probationary officers were increasingly being 'trained' by inexperienced peers, the 
traditional officers' script lost its mode of transmission. For probationary and new officers this meant it was difficult to gain trust from fellow officers or respected authority from inmates. For longer serving officers this led to frustration at the inability of the probationary and new officers to function as they should, and their withdrawal from the officer group. For the officer body as a whole, division and fragmentation emerged between the service length groups.

As such, all officers' 'lags' became progressively more difficult and confusing (see chapter six). Where in the past there had been respected authority from rank, there was now increasing animosity and resentment towards management with a clear dividing line preventing both communication and contact. Rather than one seamless body of workers seeking to achieve the same goals for the organisation, there were now two disparate groups. As a result of the changes, the officers' antithetical other was no longer inmates, but, rather, management. As with any group that is in opposition to another, the subordinate group began to subvert the superordinates' goals through secondary adjustments, in contrast to the expectation that the superordinates had had of a new, dynamic, more professional prison service. At the same time, the relational line between officers and inmates became more fluid and contingent. This led to confused interactions and uncertainty and stress within the officer group. On top of this, the traditional white male officer group was expected to accommodate women officers within its corps. Their diversity closed culture did not know how to respond to them. On the one hand, it was recognised that women could 'calm inmates down', but, at the same time, the male officers were suspicious of them because of their perceived ability to secure preferred task secondment through sexual favour. They also resented the way in which women officers did not have to perform the 'real' dirty work of the prison, such as strip searches. Because of this distrust, women had not been able to effectively insert themselves into the dynamics of the prison, but remained outsiders, making theirs an exceedingly difficult lag. Conversely, ethnic officers effectively gained much more power in the prison, successfully challenging the dominance of the Pakeha prison officers. For longer serving Pakeha officers this led to more resentment, on top of that which they felt about restructuring, and meant that they were much more likely to work together and exclude others. Newer Pakeha officers found themselves as marginalised others, a reaction to the feeling given to them that they were unwanted outsiders in the prison, both by longer serving officers and by the increasingly 
powerful ethnic minority officers. They thus tended to be perfunctory rather than dynamic in their approach to their work and to their relationships with colleagues.

Unit management had been seen as the panacea by Head Office. The intention had been that it would cement the effects of the new policy and structural changes and prevent the coalescence of the old style unity between prison officers. However, officers effectively manipulated these arrangements, producing more divided and independent groups of officers with varied demography, work practices and scripts (see chapter seven). Rather than the intended efficient competition between units, disparate cultures emerged with behaviours far more insidious than the traditional culture that these had replaced. The prison officer body was too weak in the aftermath of the changes to prevent these fragmentations and distortions. Management, too, now much more distant from the day-to-day reality of prison life, could do little to correct the disruptive elements that were occurring. Although a semblance of control was regained, that the unit cliques had been able to develop and flourish was an indicator of what the destruction of the old style prison officer unity and cohesion was capable of unleashing.

The role of the prison officer, their working arrangements, indeed almost everything they had known had changed. The reorganised prison, and all that this brought with it, was a reality that all officers had to adapt to. As increasing numbers of new recruits were being attracted to the job by the promise of a rewarding, challenging and exciting career, everyday life in the prison had become much more different, certainly, but also much more unpredictable and unstable. As a result of this tension, prison officers adopted strategies to make their work understandable in such a way that each day was different while each day remained the same (see chapter eight). Rather than the exciting challenging existence the prison authorities had intended, the everyday lives of prison officers remained mundane and banal.

Of course, one should not romanticise the previous prison service occupational culture. It could be perverse-based on sexist, racist and machismo values-and was very insular and suspicious of outsiders. However, as it weakened and faded during the course of restructuring, what it eventually metamorphosed into was something very far from what had been expected and, in many ways, something more insidious. 
What there had been previously was an occupational structure which, although undemanding, was certain and secure, where everyone knew their place, where there was uniformity across the prison, mutual dependency and reciprocity and a respected authority at each rank within the service. What had actually replaced it? A divided prison, with highly differentiated units, both in terms of what they did and the behaviour and scripts of prison officers within them, and differentiated access to training - all of which was divorced from any day-to-day contact with management. The prisons became much more difficult and confusing places to run. The loss of stability, cohesion and unity within the prison service was already beginning to haunt Head Office at the time I saw the first indications of the extent of what could go wrong with the prison with the unit corruption. This was later reflected in a range of other violent and corrupt incidents within the New Zealand prisons.

Overall, the lesson from this research is that superimposing reform on an organisation, in this case the prison service, is fraught with problems. Without the consent of those whom it is imposed on, it may only generate a further succession of problems and unintended consequences rather than the expected and desired changes. In a context of massive social change and reorganisation, a changing inmate population, new recruitment initiatives and attempts to professionalise prison officers, subverting entrenched officer culture produced, at best, disenchantment, disillusionment and division; at worst, it led at times to an out of control prison service and prison life. 


\section{APPENDICES}




\section{$\underline{\text { APPENDIX A }}$}

\section{HEC APPROVAL NOTIFICATION}




\section{VICTORIA UNIVERSITY OF WELLINGTON \\ Te Whare Wananga o te Lipoko o te $\mathrm{Kka}$ a Maut}

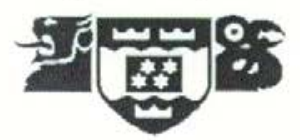

MEMORANDUM

TO:

Andrea Napier

FROM: $\quad$ Professor Graeme Kennedy

Convener, Human Ethics Committee

DATE: 13 June 1997

\section{SUBJECT: ETHICAL APPROVAL: PRISON OFFICER CULTURE}

Your application for ethical approval has been considered by the Human Ethics Committee and is approved. Members of the committee were very impressed by the thoroughness of your application and wish to congratulate you and your supervisor for its clarity and high quality. The committee wishes you every success in carrying out this interesting piece of research.

Date of Approval

12 June 1997

Duration

January 2000

Conditions

nil

Other Comments

the thesis supervisor's name should appear as a contact person on the information sheet

Graeme Kennedy

Convener

HUMAN ETHICS COMMITTEE

Figure A.1 Copy of the Victoria University of Wellington Human Ethics Committee approval notification. 


\section{APPENDIX B}

\section{FORMAL CONTRACT}




\section{THE Work OF NeW ZEALANd PRISON OFFICERS}

\section{RESEARCH CONTRACT}

The following issues concerning responsibilities and liabilities have been discussed and agreed upon by the undersigned parties in relation to the research on The Work of New Zealand Prison Officers being conducted by Andrea Napier under the academic supervision of Dr John Pratt.

\section{Issue One: Confidentiality of information gathered by Andrea Napier.}

All information pertaining to individual staff members gathered by Andrea during the course of her research remains confidential to Andrea. She will not discuss this information with any other party, including staff and management. In terms of staff, the only exception to this complete confidentiality is if Andrea is required to present as a witness to a critical incident, or complete a standard prison report regarding a critical incident, whereby only the details of the specific incident will be discussed. In terms of inmates, the above applies, however, in addition, Andrea is to report any information from inmates regarding potential breaches of institutional security and issues of personal safety to self (inmate) and others. Such information from inmates is to be directly reported to [Site Manager's name].

Issue Two: Behaviour and responsibility for basic safety and security by Andrea Napier.

General: Andrea is to comply with the basic safety and security procedures outlined during the induction session with [Instructor's name] and in the booklet entitled Security Awareness Programme For Non-Custodial Staff issued during that training.

Security: Andrea is not to be issued, nor handle, any set of institutional keys for any purposes. Andrea is in no way responsible for security within the institution, with the above exception of reporting information from inmates regarding potential breaches of security. During an emergency (such as fire or earthquake), the discretion of the Prison Officer Andrea is rostered on with applies.

Safety: Andrea is required to wear a personal alarm, issued by [Yellow Unit], whenever in the institution during this research period. Andrea accepts complete liability for her personal safety while in the institution during the research period. 
Neither [Target Prison's name], Department of Corrections, nor any individual staff member is in any way responsible for Andrea's safety nor her actions. Andrea must minimise, to the best of her ability, the potential of jeopardising the safety of others. For example, Andrea must maintain a suitable distance during any critical incident and ensure Prison Officers are not impeded by her presence when addressing such incidents.

\section{Issue Three: Presentation of research findings by Andrea Napier.}

Andrea is to exclude any details that could identify individuals in any work produced as a result of this research. No information is to be made publicly accessible that could jeopardise institutional security. A representative of [Target Prison's name] has the right to read the completed thesis, during a reasonable time period, prior to formal submission to Victoria University. Discussion will be entered into between that representative, Andrea, and Dr Pratt if requested, however, no party, other than Dr Pratt, has veto power over the contents of the report.

During the course of the research period, Andrea is not to discuss or report to news broadcasters (specifically newspapers, journalists, radio news, television news) specific incidents involving the institution or details regarding inmates. Andrea is able to publicly report, including via the above mediums, details of her thesis after it has been accepted by Victoria University, however, confidentiality of individual details permanently remains.

By signing this form, the following parties show they have discussed and agreed to the above conditions.

\section{[TARgET PRISON'S NAME] PRISON REPRESENTATIVE}

Signature:

Name (Print):

Date: /

\section{RESEARCH SUPERVISOR}

Signature:

Name (Print):

Date: 1

\section{RESEARCHER}

Signature:

Name (Print):

Date: / 


\section{$\underline{\text { APPENDIX C }}$}

\section{RESEARCH INSTRUMENTS}




\section{QUESTIONNAIRE}




\section{The Work Of NeW ZeALANd PRISON OfFICERS}

\section{PRISON OFFICER'S QUESTIONNAIRE}

(1) Please mark the box to indicate your gender:

$\square$ Male

Female

(2) Please mark the box to indicate the age group to which you belong:
$\square 15$ - 19 years
$\square 30$ - 34 years
$\square 45$ - 49 years
$\square 20$ - 24 years
$\square 35$ - 39 years
$\square 50$ - 54 years
$\square 25$ - 29 years
$\square 40$ - 44 years
$\square 55$ - 65 years

(3) Please mark the box or boxes to indicate which ethnic group or groups you identify with:
$\square$ NZ Maori
$\square$ Cook Island Maori
$\square$ NZ European
Samoan
$\square$ Other - Please specify:

(4) Please mark the box to indicate your current position as a Prison Officer:
$\square$ Probationary Prison Officer
$\square$ Prison Officer/ Front Line Staff
$\square$ Unit Manager
$\square$ Other - Please specify:

(5) Please mark the box to indicate the highest level of educational qualification you have obtained (not including internal qualifications):

$\square$ None

$\square$ School Certificate - Please specify number of papers 'awarded':

$\square$ Sixth Form Certificate - Please specify number of papers 'awarded':

$\square$ University Entrance

$\square$ Bursary - Please specify number of papers 'awarded':

$\square$ Seventh Form Scholarship - Please specify number of papers 'awarded':

$\square$ Polytechnic Diploma/ Certificate/ Degree

Please specify:

University Diploma/ Certificate/ Degree

Please specify:

Other - Please specify: 
(6) Please mark the box to indicate whether you have passed any internal courses:

$\square$ Yes - Please go to question 7

$\square$ No - Please go to question 8

(7) Please list the internal courses you have passed:

(8) Please state the approximate length of time (years and/or months) that you have been a Prison Officer:

(9) Please state the approximate length of time (years and/or months) that you have been at your current institution:

Note: If you have been at your current institution for a longer time period than you have been a Prison Officer, please specify your role or roles during this period:

(10) Please mark the box or boxes to indicate whether you have worked as a Prison Officer at any other New Zealand or Overseas Prison:

$\square$ No other Prisons

$\square$ Other New Zealand Prison(s) - Please specify how many:

Please specify which other New Zealand Prison(s):

Other Overseas Prison(s) - Please specify how many:

Please specify what countries:

(11) Please mark the box to indicate whether you have had any previous military experience:

$\square$ Yes - Please go to question 12

$\square$ No - Please go to question 13

(12) Please mark the box(s) to indicate which military service(s) you have had experience with:
$\square$ Army
$\square$ Navy
$\square$ Air Force
$\square$ Other - Please specify: 
(13) Please list the occupations you held before becoming a Prison Officer:

(14) Please mark the box or boxes to indicate your reasons for becoming a Prison Officer:
$\square$ The job looked interesting
$\square$ For the money
$\square$ Friends/ relatives were Prison Officer(s)
$\square$ Always wanted to be a Prison Officer
$\square$ The challenge/ excitement
$\square$ To help reduce re-offending
$\square$ Just needed a job
$\square$ Other - Please specify:
$\square$ Power

(15) Please list three things about your job that you like the BEST:

a)

b)

c)

(16) Please list three things about your job that you like the LEAST:

a)

b)

c)

)

If there are additional comments you would like to make about the questionnaire or about your life and work as a Prison Officer, please do so below. 
Each Day is Different: Prison Officers and Their Work

\section{INTERVIEW SCHEDULE}




\section{GENERALISED AREAS TO INCLUDE IN INTERVIEW}

GOAL:

- To understand how prisons work we need to understand the role of POs, which is not necessarily determined by official policy, but by local culture, which is shown by how you experience the job.

\section{THE Job Per SE.}

- Where did you find out about the job

- What did you think the job was about

- How does the job compare with your expectations/ perceptions

- Where do you see yourself going (career/ short-term)

- If you had known what working in a prison was like when you applied would you have still applied

- Would you recommend the job to friends/ family (why/ why not)

- Have the recent disturbances in other prisons ([Two National Prison's names that had recent disturbances]) changed your feelings about the job (if yes - how e.g. more dangerous/ reaffirm the nature of the job as dangerous etc.)

\section{RECRUITMENT/ QUALITIES}

- What qualities do you think makes a good PO

- What type of characteristics make a person not a good PO (average or bad PO qualities)

- Has the PO group changed since you joined the job (type of PO joining and persisting changed) - if so, how

- What do you think of the new recruitment policies (how are they different from your time)

- What do you think about the new style PO - do they fit the role/ job

- What do you think of the old style PO - do they fit the new role/ job

- Do you think the training you get prepares you for the job - what would be useful training

\section{JOB ROLE}

- What do you see your role is as a PO

- How has the role of the PO changed since you have been in the job (better or worse; easier or harder; what is expected of you; what you can/ can't do; how you're treated etc.)

- What conflicts/ dilemmas do you see existing in your job/ role as a PO

- How do you feel about the dual role of case management and containment

- Is the official role of the PO as dual a reality of the job

- Can you think of instances where the official role (joint case management \& security) is problematic/ useful

- What do you think the role should be for POs (would it be more or less stressful/ useful - why) 


\section{JOB RULES}

How clear do you think the rules of the job are (such as PPM vs. PIGO)

- Does this effect how you can go about your job (are the rules realistic/ used etc.)

\section{GENDER IsSUES - MALE PARTICIPANT}

- Do you think that women should be POs in a male prison

- Are there benefits/ negatives to having women in male prisons

- What difference has there been having women in the jail (if longer serving PO)

- What issues are there with women working in a male prison

- Do you think there are expectations for women to behave in certain ways (if yes, what are they)

- Does the female PO role differ to the male PO role (if yes, how)

- What makes a good female PO compared to a good male PO (or maybe the same)

- Do you feel women are treated any better/ worse on the job (by POs; inmates; management)

\section{GENDER IsSUES - FEMALE PARTICIPANT}

- Do you think women should be POs in a male prison

- Are there benefits/ negatives to having women in male prisons

- What do you think male POs think about women being POs in male prisons

- What issues arise for you being a female in a male prison

- Are you treated differently being a female (by: management, inmates, male POs, women POs, outsiders)

- Are there expectations for women to behave certain ways (what are they)

- Does the female PO role differ to the male PO role (if yes, how)

- What makes a good female PO compared to a good male PO (or maybe the same)

- How do you feel about the sexual joking/ innuendoes/ sexual harassment

- Where does this (use the word they use) come from

- What form does it generally take

- How do you handle this

- Would you support a woman that said she was being harassed (how - or should women put up with it as part of the job)

- Can you think of any instances where your gender or sex has been an issue for you in the job

\section{ETHNIC ISSUES}

- Does being X(what they are) make your job easier or harder (if yes, how)

- Do you think the ethnicity of a PO effects how they go about their job (who, how)

- Does it effect you

- Do you think certain ethnic groups are expected to behave in certain ways

- Have you experienced/seen this

- Is it positive or negative having such ethnic variety within the PO group (how)

- Are you treated any differently being X (what they are) by: inmates, management, other POs (if yes, how) 
- Can you think of any instances where your ethnicity has been an issue for you in the job

- If I said the term 'Bros' or 'Black Mafia' to you, what does this mean

- Their function/ role

- Membership

- Value

- Equivalent groups

\section{The Prison OfFicer Group}

- Do you perceive the prison as having some sort of culture

- Is it a prison thing or do you think officers and inmates have distinct cultures

- What makes up the culture (differences if two seen) (values; rules; language)

- Where do you think this culture comes from

- Does 'prison' culture effect the way you go about your job (if yes, how)

- Do you think it is necessary for POs to get on well as a group - why

- Do you think that there is one PO group, many sub groups, or an over-riding PO group with subgroups (what are the groups; where do you think you fit in etc.)

- Do you think there are PO types, e.g. some just do their job, some get very involved in the prison etc.

- What are they

- Where would you be

- How would you rank those types

- There appears to be a hierarchy within the PO group (is this linked to the above groups)

- What is this based on (service, ethnicity, prowess in conflict, skill in job etc.)

- How would you rank the group

- POs have said that an important element of the group is that 'friendships are forged in fire'

- What does this mean to you

- Do you agree

- Have you experienced this (if yes, how)

- Does the need to get on with other POs effect how you are able to do your job at times (examples)

- What do you think are the important rules within the PO group e.g. don't nark; don't paper etc.

- What would be the three important do's

- What would be the three important don'ts

- How important are these rules for you personally; for the job; for the PO group

- What is more important - informal rules or formal rules

- How do the informal rules and formal rules sit together (balance, mismatch etc.)

- What do you think is the function of these rules

- What happens if you don't stick to these rules (examples)

- What advice would you give someone starting out in the job (the 'to do's and not to do's')

- What would be the worst thing you could do as an officer (would others agree)

- What do you think the PO group values (e.g. information seems important) 
- Food is important - why

- A physically challenging job yet body image doesn't match - why

- What strengthens the PO group/s

- What weakens the PO group/s

\section{INMATES}

- How do you feel about inmates

- What sort of problems do you encounter dealing with inmates

- Do you feel differently about any particular inmate group, offence type (if yes, does this effect how you do your job)

- Have inmates changed since you've been in the job (if yes, how)

- Do you think the level of control POs have over inmates has changed (how, why what is better)

- Compared to prisons in other countries, POs in NZ have a lot of contact with inmates at a personal level:

- Has it always been like this in your view

- Why do you think this is

- Does it help with your role

- Does it create additional stress

- Would you prefer less/ more inmate contact

- What are the lines I often hear about

\section{MANAGEMENT}

- Do you differentiate between management levels

- What makes a good manager

- Do you think 'management' (UM and/or upper) is supportive of you/ POs

- How has 'management' changed since you've been in the job

- Do you think management (general for their interpretation) supports POs (how/ how not)

- If you were given the opportunity to change aspects of management - what would you do

- If you were going to rank the support you feel from groups other than POs, how would you rank: management, inmates, public, ancillary staff

- Are there any other groups that you would say are supportive of POs

\section{OUTSIDERS}

What do you think is the general public opinion of your job

- Why do you think people think that about your job

- Prisons are in the news a lot lately. Do you think the media treats POs fairly (how, why - would other POs agree)

- If an outsider asked, what would you tell them you did for a job (e.g. tell them something else; how would you describe the job etc.)

- Do you socialise mainly with POs or with other people

- Why do some/ you socialise mainly with POs

- What do you think your family think about you being a PO 


\section{VIOLENCE}

- Do you have any fears about violence while working in the prison (what, when, who, why)

- How do you combat these fears

- Do you think they are common fears

- Have you ever been assaulted while working in prison (examples)

- How common do you think inmate to officer violence is

- If low - why the fear (nature of violence; unpredictability)

- Generally under what conditions does inmate to officer violence occur

- How do you feel about officer to inmate violence

- Why do you think it occurs

- When is it justifiable

- Why would some POs view it as necessary

- Has it changed

- How often would you say it occurred

- What is it's function

- If I said the term 'Goon Squad' to you, what would it mean

- Would you say [Target Prison’s name] has one

- Which groups would be in it

- What is their purpose

- Is it generally known about (does management know - do they attempt to stop it)

\section{INFORMAL DISCIPLINE}

- What types of informal discipline have you seen in the prison (examples)

- How do you feel about the use of informal discipline

- Who does it

- Is it necessary/ useful

- Has it changed

- How often would you say it occurred

- What would you say is the main reason violence and informal discipline are sometimes used

\section{STRESS}

- What sort of stresses are there in the job

- How are they caused

- How do you cope with them

- Who supports you through stressful times

- What would make the POs job safer; more satisfying; more effective

- What is your biggest fear in terms of bad/ stressful things occurring on shift 


\section{EXPERIENTIAL}

- How would you describe a typical day at work

- What sorts of things do you fear could occur on shift

- What equates to a good shift

- What are things that you remember best about your experience as a PO

- What major negative things do you remember about your experience as a PO

- Do you think the job changes people as people, that is, have you noticed changes in other POs since being on the job - how; why

- Does working in the job change some people and not others (who; how; why)

- Do you think the job changes you in any way - how; why

\section{Anything YOU want to add}




\section{$\underline{\text { APPENDIX D }}$}

PRISON MEMORANDUM 


\section{MEMORANDUM}

TO:

Unit Managers and Unit Staff

FROM: $\quad$ Acting General Manager

DATE: [date]

SUBJECT: $\quad$ Andrea Napier

On [date], Andrea Napier will be commencing a research programme on the understanding and the explanation of the work of Prison Officers.

Andrea is a Criminology graduate student at Victoria University.

There will be three stages of research:

Stage 1 involving the distribution of a questionnaire to all Prison Officers at [Target Prison's name]. Prison Officers in [Units' names] will receive their questionnaire in the next 3 weeks.

At a later date questionnaires will be distributed to [Units’ names].

Stage 2 will be observing Prison Officers at work.

Stage 3 will involve interviewing 40 Prison Officers selected from those who have returned the questionnaire.

Andrea will then spend a further four weeks for informal discussion and comments.

Whilst she is doing this research Andrea will be working initially in [Units' names] alongside Prison Officers on their normal rostered duties. She will not be a Prison Officer, just an observer.

Please find attached copies of the rosters for Andrea from [date - date of initial familiarisation period].

[Signature]

[Acting General Manager's name]

Figure D.1. Representation of the prison memorandum. 


\section{APPENDIX E}

\section{SCHEMATIC IMAGES OF THE PRISON DESIGNS ${ }^{174}$}

${ }^{174}$ For security reasons limited description has been provided and parts of the images have been modified. 


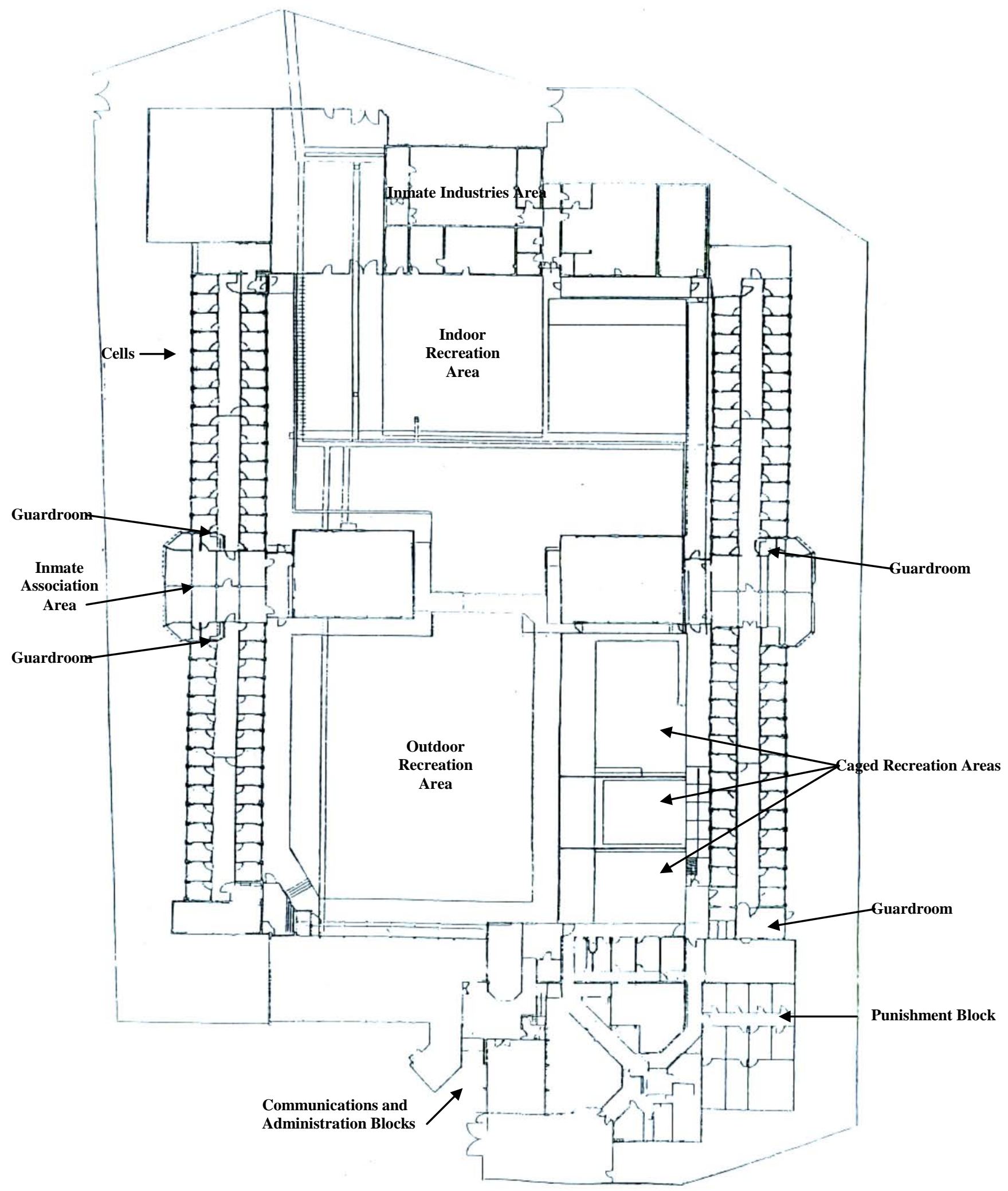

Figure E.1. Schematic image of Alpha Jail. 


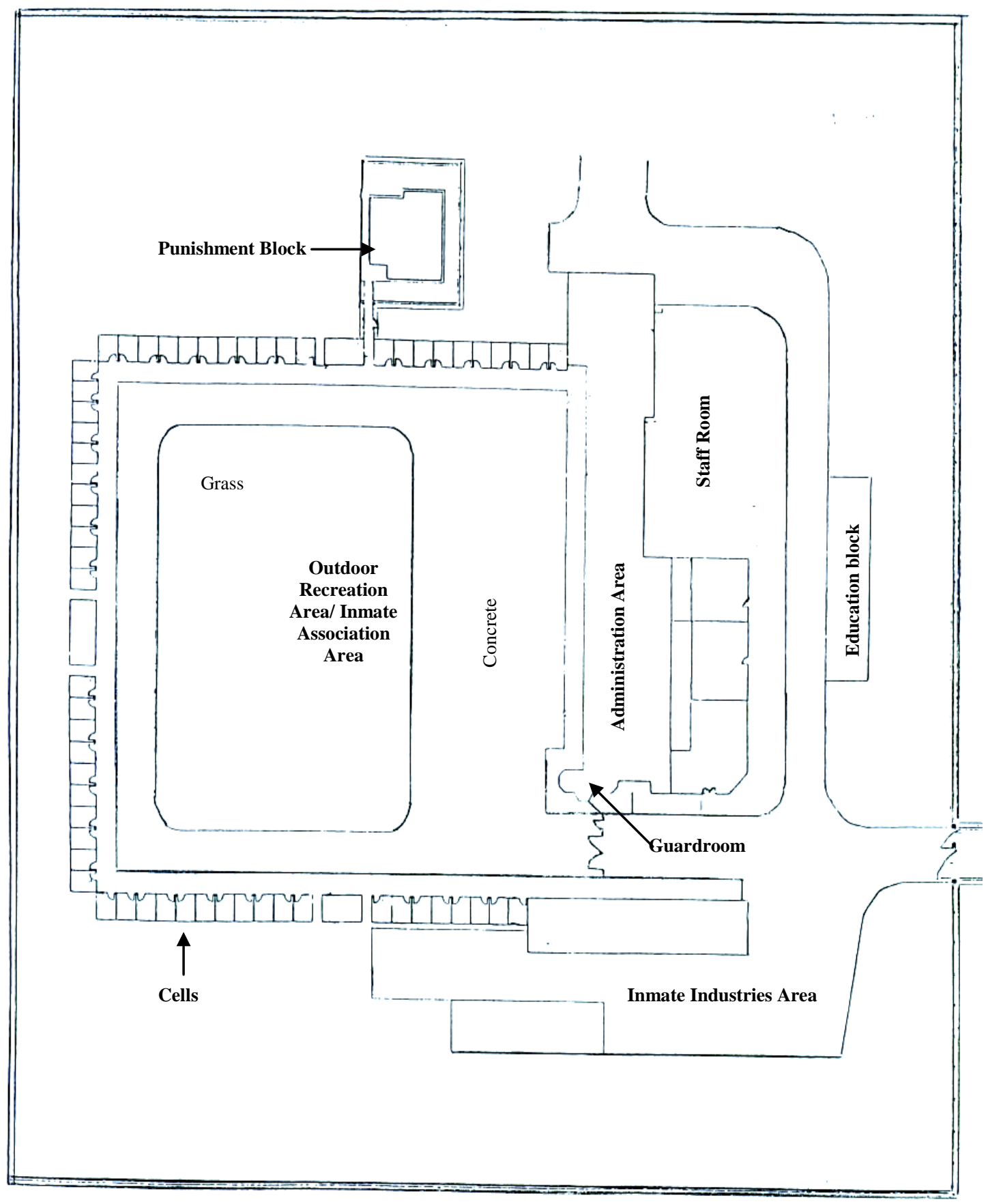

Figure E.2. Schematic image of a Beta Jail unit. 


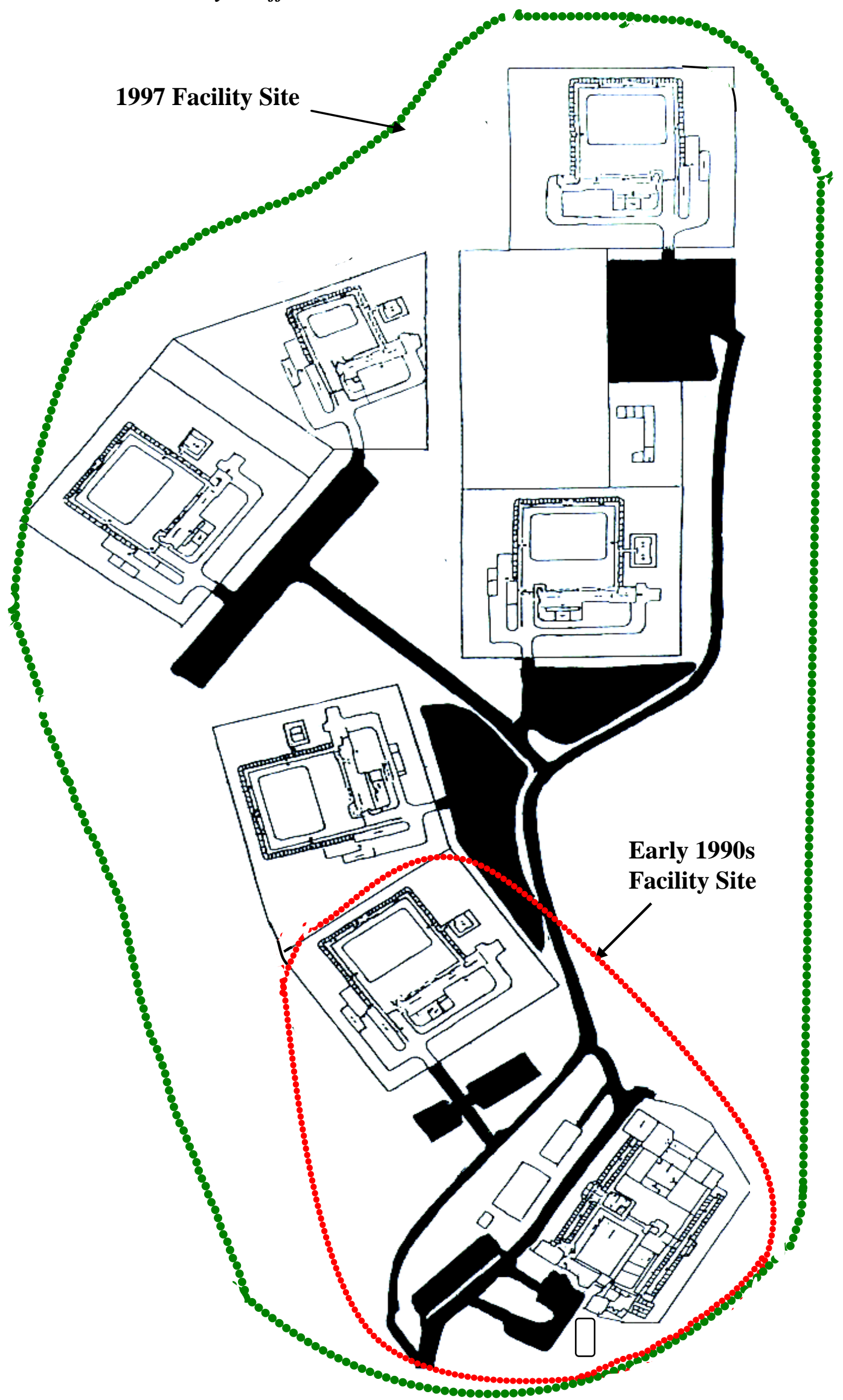

Figure E.3. Schematic image showing the early 1990s target facility site compared to the 1997 target facility site. 


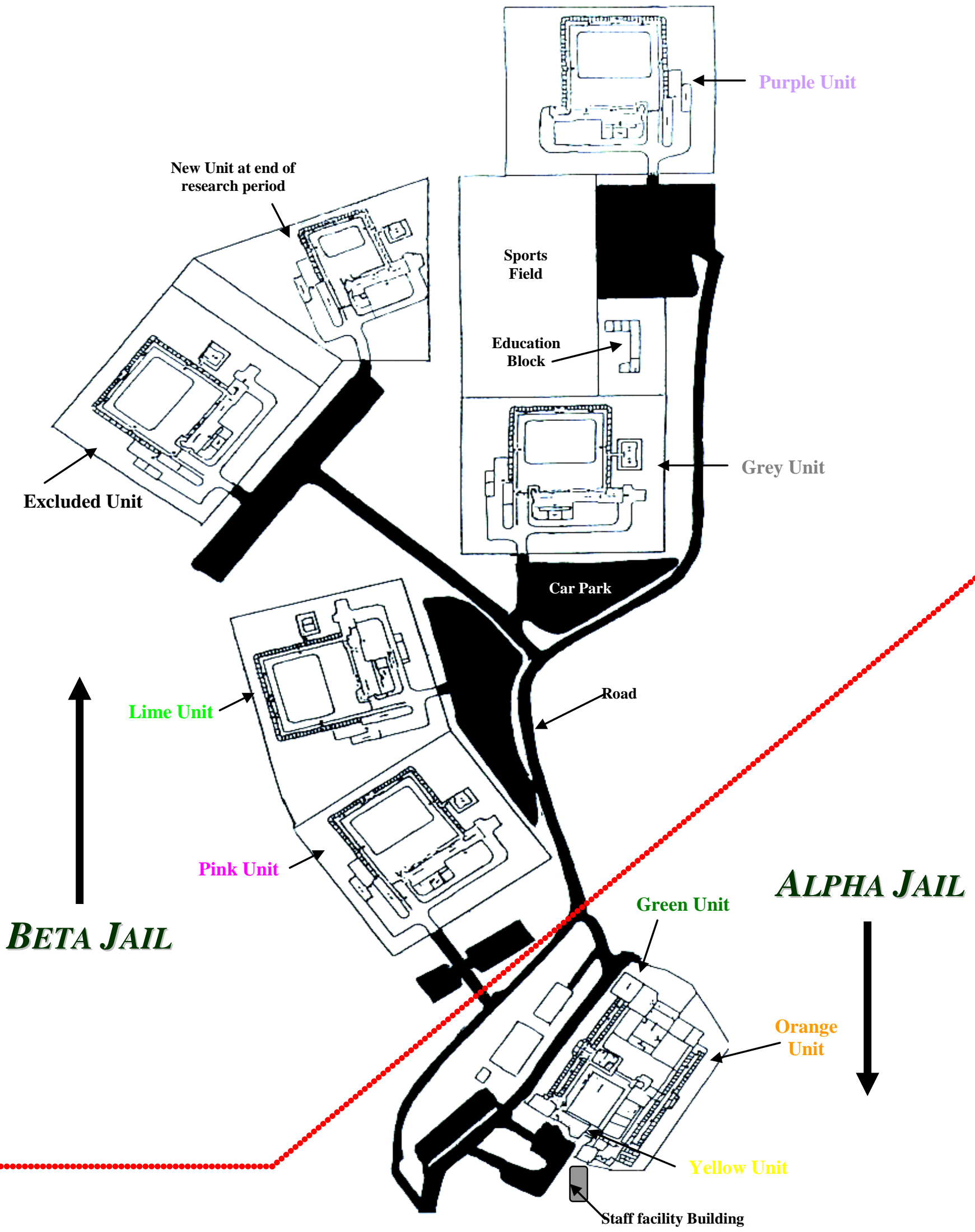

Figure E.4. Schematic image of the complete target facility at the end of the research period. 


\section{$\underline{\text { APPENDIX F }}$}

\section{MAPS OF NEW ZEALAND SHOWING PRISON REGIONS AND LOCATIONS $^{175}$}

\footnotetext{
${ }^{175}$ Note that the names of specific prisons have changed over time. Similarly, several prisons have colloquial names. The names used on official documentation as at the time of my research have been used here.
} 


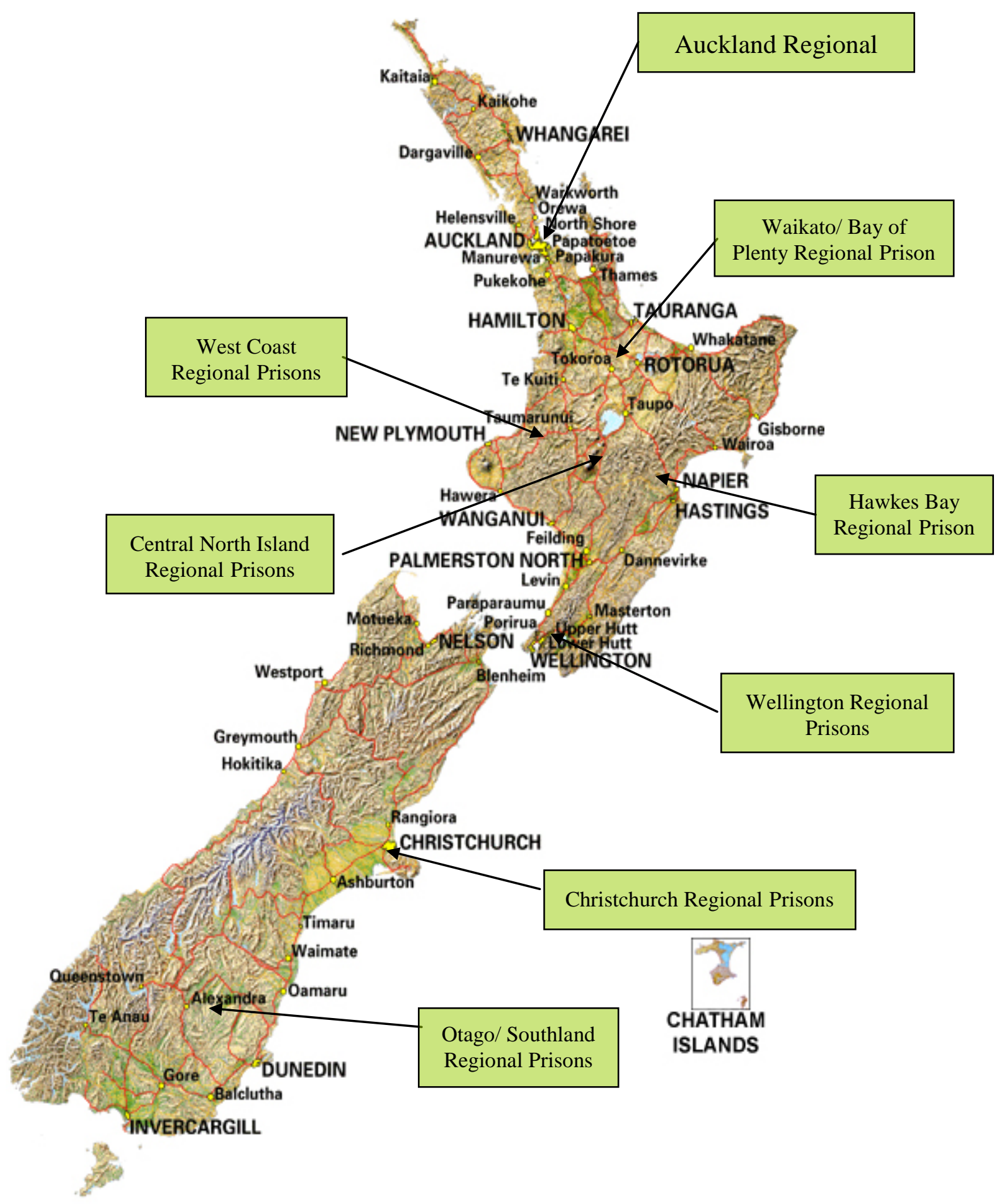

Figure F.1. Map of New Zealand showing the approximate prison regions. 


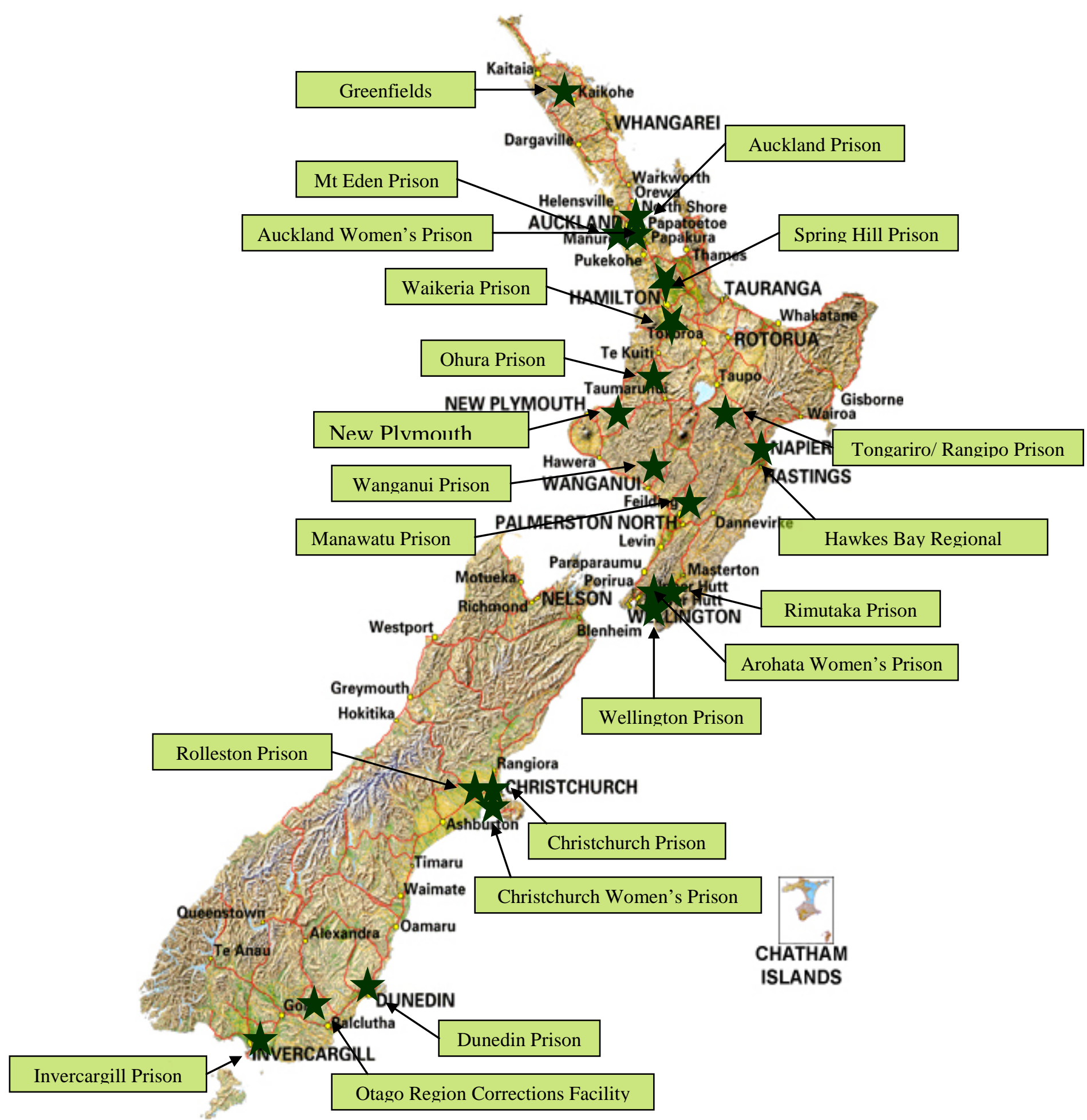

Figure F.2. Map of New Zealand showing the approximate prison locations. 


\section{APPENDIX G}

\section{General Population GROWTH COMPARED TO PRISON POPULATION GROWTH}




\section{Prison inmates}

Numbers in prison per 10,000 mean population

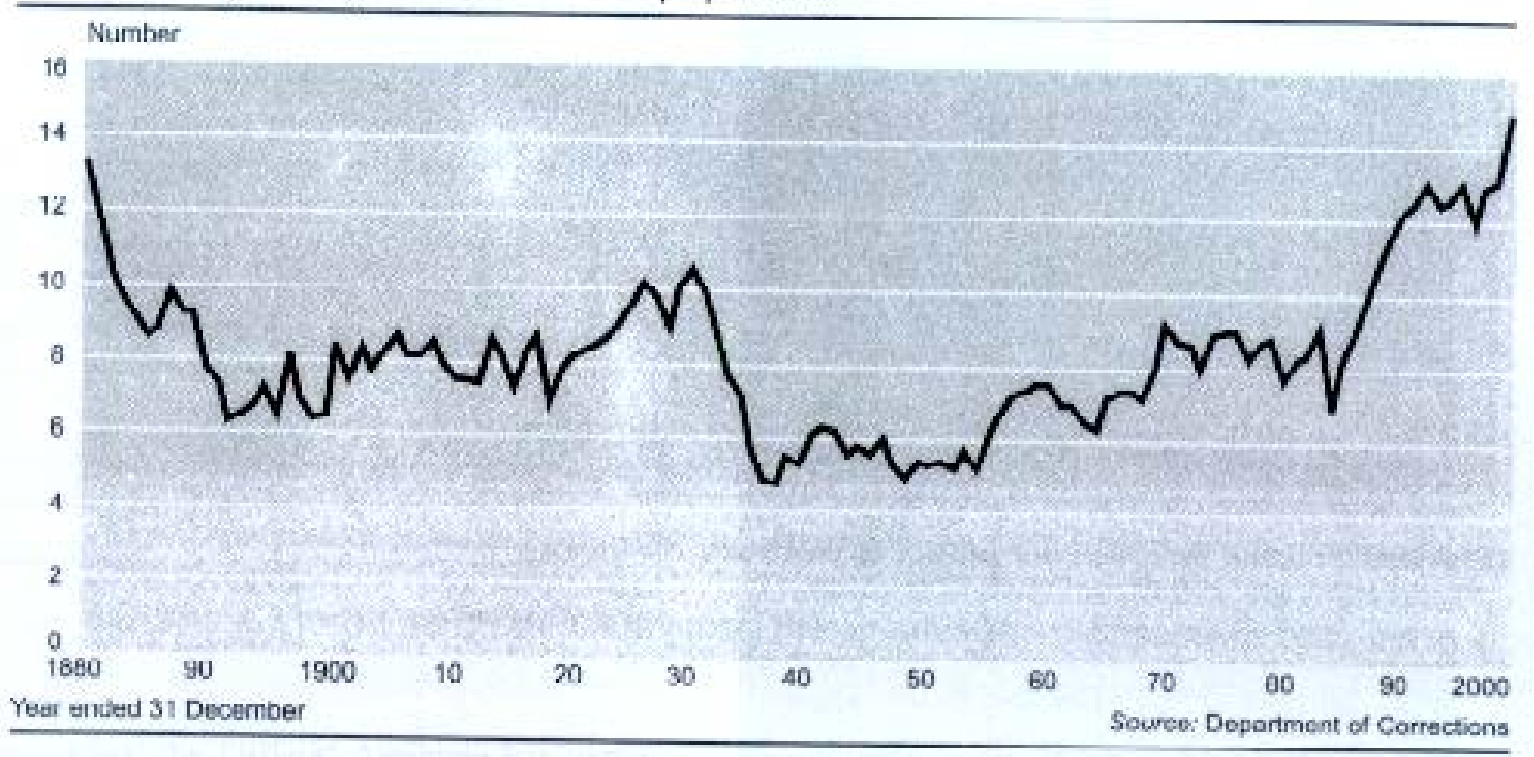

Figure G.1. Graphical image demonstrating the trend of inmate population per 10,000 mean general population 1880 through $2000^{176}$.

${ }^{176}$ Source: New Zealand Official Yearbook (2002, p. 252). 


\section{Table G.1}

Total Population Number for Censuses 1858 Through 2001 Demonstrating the Population Growth of New Zealand ${ }^{177}$

\begin{tabular}{|c|c|}
\hline Census Dates & Total Population \\
\hline 1858 & 115,461 \\
\hline 1874 & 344,985 \\
\hline 1878 & 458,007 \\
\hline 1881 & 534,030 \\
\hline 1886 & 620,451 \\
\hline 1891 & 668,652 \\
\hline 1896 & 743,214 \\
\hline 1901 & 815,862 \\
\hline 1906 & 936,309 \\
\hline 1911 & $1,058,313$ \\
\hline 1916 & $1,149,225$ \\
\hline 1921 & $1,271,667$ \\
\hline 1926 & $1,408,140$ \\
\hline 1936 & $1,573,812$ \\
\hline 1945 & $1,702,329$ \\
\hline 1951 & $1,939,473$ \\
\hline 1956 & $2,174,061$ \\
\hline 1961 & $2,414,985$ \\
\hline 1966 & $2,676,918$ \\
\hline 1971 & 2,862,630 \\
\hline 1976 & $3,129,384$ \\
\hline 1981 & 3,143,307 \\
\hline 1986 & $3,263,283$ \\
\hline 1991 & 3,373,926 \\
\hline 1996 & 3,618,303 \\
\hline 2001 & $3,737,277$ \\
\hline
\end{tabular}

${ }^{177}$ Source: New Zealand Official Yearbook (2004, p. 82). 
Table G.2

Inmate Population Figures Demonstrating Inmate Population Growth From 1874 Through $2001^{178}$

\begin{tabular}{|c|c|c|}
\hline Year & Number of Inmates & Data Source \\
\hline 1874 & 533 & Census $(1875, p .161)$ \\
\hline 1878 & 682 & ibid (1880, p. 295) \\
\hline 1881 & 686 & ibid (1882, p. 257) \\
\hline 1886 & 625 & New Zealand Official Yearbook (1895, p. 216) \\
\hline 1891 & 534 & idem \\
\hline 1896 & 524 & Census $(1897, p .330)$ \\
\hline 1900 & 568 & New Zealand Official Yearbook (1902, p. 285) \\
\hline 1901 & 713 & ibid (1903, p. 186) \\
\hline 1906 & 832 & Census $(1907$, p. 385) \\
\hline 1911 & 873 & New Zealand Official Yearbook (1913, p. 278) \\
\hline 1916 & 861 & Census $(1918, p .94)$ \\
\hline 1920 & 1,060 & New Zealand Official Yearbook (1922, p. 170) \\
\hline 1924 & 1,247 & ibid (1926, p. 254) \\
\hline 1928 & 1,484 & ibid (1930, p. 264) \\
\hline 1935 & 1,150 & ibid (1937, p. 151) \\
\hline 1940 & 895 & ibid (1943, p. 127) \\
\hline 1945 & 1,040 & ibid (1948, p. 171) \\
\hline 1950 & 1,083 & ibid (1953, p. 215) \\
\hline 1955 & 1,154 & ibid (1957, p. 260) \\
\hline 1960 & 1,828 & ibid (1962, p. 266) \\
\hline 1965 & 1,702 & ibid (1967, p. 256) \\
\hline 1970 & 2,365 & ibid (1972, p. 248) \\
\hline 1975 & 2,752 & ibid (1977, p. 238) \\
\hline 1980 & 2,801 & ibid (1982, p. 251) \\
\hline 1985 & 2,235 & ibid (1988, p. 301) \\
\hline 1990 & 3,990 & ibid (1992, p. 188) \\
\hline 1995 & 4,448 & ibid (1997, p. 268) \\
\hline 2001 & 5,780 & ibid (2004, p. 198) \\
\hline
\end{tabular}

\footnotetext{
${ }^{178}$ Sources of this data garnered their data from a variety of specified and non-specified sources, used various uncited or unstipulated criteria, and therefore should be viewed as approximates of population numbers. Depending on the source, the data could vary widely for a given year - the most likely correct figure was used in such instances. More accurate data was not possible.
} 


\section{$\underline{\text { APPENDIX H }}$}

\section{Prison OfFICER NuMBers}




\section{Table H.1}

Census Data for Demonstrating Numbers of Persons Identifying as Being Employed in Penal Establishments 1874 Through $2005^{179}$

\begin{tabular}{|c|c|c|}
\hline Census Dates & $\begin{array}{c}\text { Total Prison Officer } \\
\text { Population }\end{array}$ & Source \\
\hline 1874 & 107 & Census $(1875, p .155)$ \\
\hline 1878 & 135 & ibid $(1880, p .289)$ \\
\hline 1881 & 150 & ibid (1882, p. 247) \\
\hline 1886 & 149 & ibid $(1886, p .261)$ \\
\hline 1891 & 122 & ibid (1891, p. 245) \\
\hline 1896 & 123 & ibid $(1897, p .315)$ \\
\hline 1906 & 145 & ibid (1907, p. 366) \\
\hline 1911 & 147 & ibid (1911, p. 433) \\
\hline 1916 & 169 & ibid $(1918, p .131)$ \\
\hline 1936 & 188 & ibid $(1946, p .48)$ \\
\hline 1945 & 232 & ibid (1948, pp. 47 \& 58) \\
\hline 1951 & 182 & ibid (1954, p. 29) \\
\hline 1961 & 203 & ibid (1965, p. 99) \\
\hline 1966 & 283 & ibid (1969, p. 102) \\
\hline 1971 & 638 & ibid $(1974, p .81)$ \\
\hline 1976 & 932 & ibid (1980, p. 147) \\
\hline 1981 & 1116 & ibid (n.d., p. 138) \\
\hline 1986 & 1281 & ibid (1988, p. 124) \\
\hline 1989 & 1622 & Ministerial Committee (1989, p. 76) \\
\hline 2005 & 2800 & Department of Corrections (2005b) \\
\hline
\end{tabular}

${ }^{179}$ Depending on the detail of data breakdown within the census, these figures have been limited as much as possible to those of warder/ officer rank. 


\section{Table H.2}

Data $^{180}$ Demonstrating Percentage Increase of the Total National Prison Officer Group Between Census Dates and Total National Officer to Inmate Ratio ${ }^{181}$ for Each Census Date 1874 Through 2005

\begin{tabular}{|c|c|c|c|c|}
\hline \multirow{2}{*}{$\begin{array}{c}\text { Census Dates }^{\mathbf{a}} \\
1874\end{array}$} & \multirow{2}{*}{$\begin{array}{c}\begin{array}{c}\text { Percentage Increase of Prison } \\
\text { Officer Group Relative to Preceding } \\
\text { Date Figure }\end{array} \\
\text { (Base) }\end{array}$} & \multicolumn{3}{|c|}{$\begin{array}{l}\text { Ratio Demonstrating } \\
\text { Officer: Inmates }\end{array}$} \\
\hline & & 1 & $:$ & 5.0 \\
\hline 1878 & $+27 \%$ & 1 & : & 5.1 \\
\hline 1881 & $+11 \%$ & 1 & : & 4.6 \\
\hline 1886 & $-0.5 \%$ & 1 & : & 4.2 \\
\hline 1891 & $-18 \%$ & 1 & : & 4.4 \\
\hline 1896 & $+1 \%$ & 1 & : & 4.3 \\
\hline 1906 & $+18 \%$ & 1 & : & 5.7 \\
\hline 1911 & $+1 \%$ & 1 & : & 5.9 \\
\hline 1916 & $+15 \%$ & 1 & : & 5.1 \\
\hline 1936 & $+12 \%$ & 1 & $:$ & 6.1 \\
\hline 1945 & $+24 \%$ & 1 & : & 4.5 \\
\hline 1951 & $-22 \%$ & 1 & : & 6.0 \\
\hline 1961 & $+11 \%$ & 1 & : & 9.0 \\
\hline 1966 & $+39 \%$ & 1 & : & 6.0 \\
\hline 1971 & $+225 \%$ & 1 & : & 3.7 \\
\hline 1976 & $+46 \%$ & 1 & : & 3.0 \\
\hline 1981 & $+20 \%$ & 1 & : & 2.5 \\
\hline 1986 & $+15 \%$ & 1 & : & 1.7 \\
\hline 1989 & $+27 \%$ & 1 & : & 2.4 \\
\hline 2005 & $+73 \%$ & 1 & : & $2.1^{\mathrm{d}}$ \\
\hline \multicolumn{5}{|c|}{$\begin{array}{l}\text { a = Note that for the years } 1936 \text { \& } 1951 \text { through } 2005 \text { the data dates were one year out. It is } \\
\text { proposed that the figures, while not precise, remain representative for discussion purposes. } \\
b=\text { Apart from } 1886 \text {, figures rounded to whole number. } \\
c \text { = Figures rounded to one decimal place. } \\
d=\text { While this figure may seem low in comparison to previous periods, it should be noted that } \\
\text { an international comparison demonstrated the New Zealand ratio remained high. For example } \\
\text { in 1999/2000 the ratio for Canada was } 1: 1.1 \text {; Australia was 1:1.8; England was 1:1.5; and } \\
\text { Scotland and Wales was } 1: 1.2 \text { (Department of Corrections, 2001b, p. 23). }\end{array}$} \\
\hline
\end{tabular}

\footnotetext{
${ }^{180}$ Raw data used for the calculations to attain the figures presented in this table was derived from tables G.2 and H.1. The sources of this original data were varied and therefore should be read as an indication of trends rather than precise data sets.

${ }^{181}$ It is acknowledged that there will be prison and regional variation of staff to inmate ratios; however, data here is intended to demonstrate the overall trend.
} 


\section{APPENDIX I}

\section{RELEVANT WORKFORCE FIGURES POST 1940}




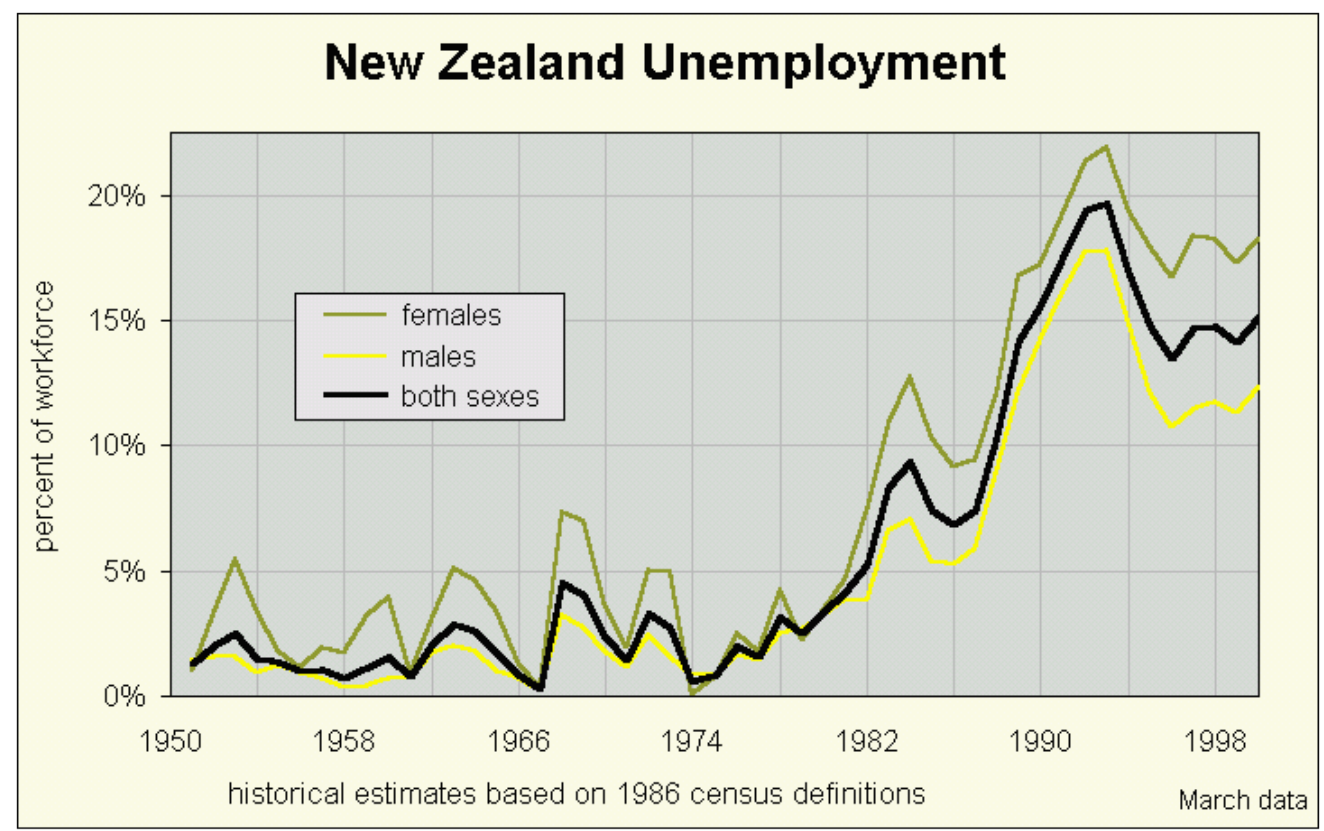

Figure I.1. New Zealand unemployment figures post-War demonstrating the 1960 s as a period of change ${ }^{182}$.

\section{Declining incomes}

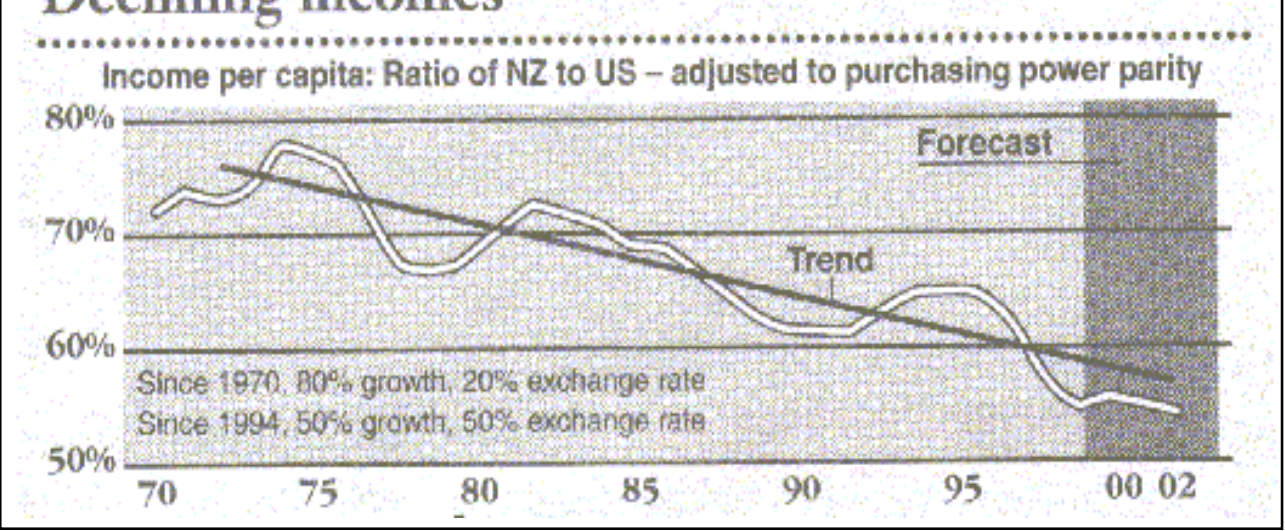

Figure I.2. New Zealand income ratio demonstrating downward trend from the $1970 \mathrm{~s}^{183}$.

182 Source: Rankin (2006a).

183 Source: Rankin (2006b). 


\section{APPENDIX J}

\section{REgiONAL MANAGERS BACKGROUNDS}




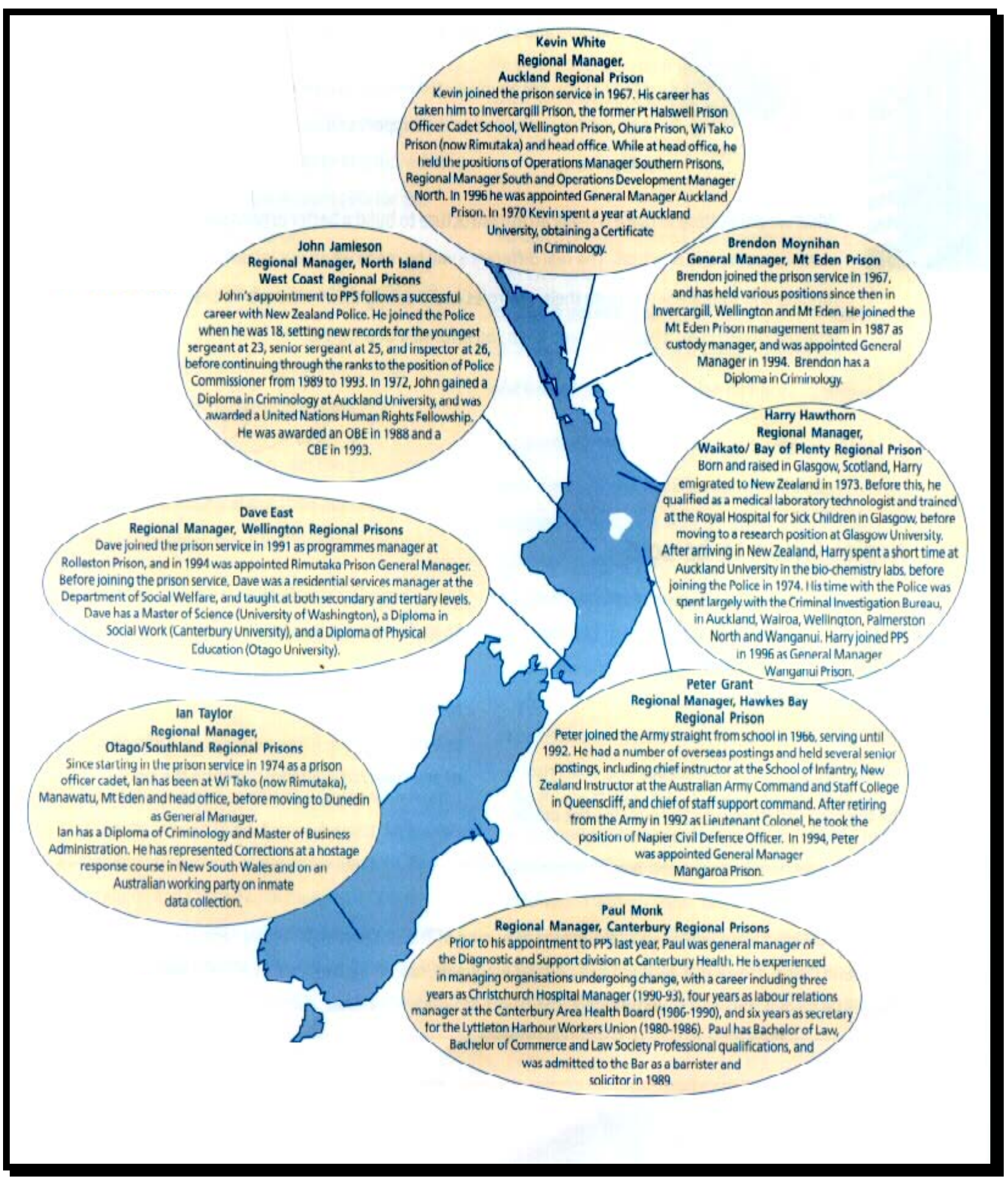

Figure J.1. Previous career biographies of the Regional Managers as at the time of research ${ }^{184}$.

${ }^{184}$ Image sourced from the Department of Corrections (1997g, p. 1). 


\section{APPENDIX K}

\section{ADDitional NoTES FOR CAREER STRUCTURE FIGURES}




\section{SUPPLEMENTARY NOTES FOR FIGURE 1.1}

Probationary OfFicers: Each new recruit was termed a probationary officer for the first 12 months of service. Four weeks was spent on a residential course at the Staff College in Trentham culminating in the 'Preliminary Officers Examination'. A pass was required to progress beyond the basic officer grade.

THIRD OFFICERS: Promotion up to and including first rank officer formally required a pass in the 'Intermediate Officers Examination', albeit not essential. Progress was formally achieved through open competition for vacancies, however, length of service contributed greatly to this decision process.

SECOND OFFICERS: An intermediary level, typically attained as a progress of length of service.

FIRST OFFICERS: A pass in the 'Senior Officer Examination' was formally required to advance beyond first rank officer grade, although again, this was not essential. The majority of long-serving officers ceased progression at this stage.

Prison InSTRUCTORS: These officers have an industries management role in addition to their custodial one. Generally employed for their industries capability and remained in that role.

DIVISIONAL OFFICERS: Roles varied between different institutions. In some cases they had a management type role (similar to the later Unit Managers role). In other institutions they performed roles to directly meet inmate needs (similar to what case officers do under the case management system). Theoretically, depending on the size of the institution, such officers could be first, second, or third rank. Typically they were derived from the first rank officers.

ASSISTANT SuPERINTENDENT: Depending on the size of the institution there may have been none to one or more of this rank. Typically this rank, as with Deputy Superintendent, was derived from the divisional officer rank or first officer group.

DEPUTY SUPERINTENDENT: Depending on the size of the institution, there may or may not have been a deputy superintendent.

SUPERINTENDENT: The person ultimately responsible for the control, management, order and discipline of the institution. Typically this person derived from the preceding rank positions. 


\section{SUPPLEMENTARY NOTES FOR FIGURE 1.2}

Probationary Officers: Similar to pre-1990, new recruits were on probation for the first 12 months. Four weeks were spent on a residential course at the Staff College in Trentham culminating in the 'Probationary Prison Officers Examination'. A further two weeks was spent at the college or at their prison site training in $C \& R$ procedures.

PRISON OFFICERs ${ }^{185}$ : All previous ranks were flattened down to the one official title. Responsible to the Unit Manager, although, under the direct supervision of the Unit IC.

Prison InSTRUCTORS: Similar to pre-1990, although more movement between prison officers that were primarily custody being seconded as instructors.

2IC OR IC OR UNIT IC: An informal role with roster privileges attached. Militarised terminology referring to 'second in command'. This officer was designated as 'team leader' by the Unit Manager to oversee the unit, and was responsible for the handover to the next shift.

UNIT MANAGER: May or may not have originated from the floor. Length of service is no longer a variable in promotion to this position. Responsible for the complete management of a given unit (staff, inmates, budget and so on). Accountable to the Manager Custody.

Note that there are more managers at this middle level than provided here. The following are the managers that officers have direct associations with. All managers at this level are accountable to the General Manager (who is responsible to the Regional Manager). All management positions have open market competition.

MANAGER Administration: Typically from a financial management background such as an accountant. Responsible for financial and other administrative functions of the entire institution.

MANAGER Custody: Responsible for the safe and humane containment and movement of inmates.

Manager Programmes: Typically a programmes specialist such as a psychologist. Responsible for the effective operation of case management.

GENERAL MANAGER: Responsible for the overall functioning of the institution.

REgIONAL MANAGER: Responsible for the overall functioning of the institutions within their designated region. Accountable to the Department of Corrections. See Figure J.3 for backgrounds of Regional Managers at the time of the current research.

\footnotetext{
${ }^{185}$ After the research period, this was formally divided into Correctional Officer (CO) and Principle Officer (PO), to acknowledge progress through the NZQA framework qualifications.
} 


\section{APPENDIX L}

\section{Proportions of MALe} AND FEMALE STAFF ACROSS INSTITUTIONS 
Table L.1

Percentage of Male Versus Female Prison Officer Staff Across Institutions ${ }^{186}$

\begin{tabular}{lccc}
\hline \multicolumn{1}{c}{ Prison } & $\begin{array}{c}\text { Total Staff } \\
\text { Number }\end{array}$ & $\begin{array}{c}\text { Percentage of } \\
\text { Male Staff }\end{array}$ & $\begin{array}{c}\text { Percentage of } \\
\text { Female Staff }\end{array}$ \\
\hline Auckland East Prison & 119 & $86 \%$ & $14 \%$ \\
Auckland West Prison & 86 & $77 \%$ & $23 \%$ \\
Christchurch Prison & 179 & $84 \%$ & $16 \%$ \\
Rolleston Prison & 124 & $66 \%$ & $34 \%$ \\
Ohura Prison & 23 & $83 \%$ & $17 \%$ \\
Tongariro Prison & 159 & $72 \%$ & $28 \%$ \\
Hawkes Bay Prison & 153 & $78 \%$ & $22 \%$ \\
Mt Eden Prison & 181 & $77 \%$ & $23 \%$ \\
Dunedin Prison & 21 & $90 \%$ & $10 \%$ \\
Invercargill Prison & 21 & $90 \%$ & $10 \%$ \\
Waikeria Prison & 228 & $85 \%$ & $15 \%$ \\
Manawatu Prison & 57 & $89 \%$ & $11 \%$ \\
New Plymouth Prison & 36 & $83 \%$ & $17 \%$ \\
Wanganui Prison & 118 & $90 \%$ & $10 \%$ \\
Arohata Women's Prison & 37 & $43 \%$ & $57 \%$ \\
Rimutaka Prison & 144 & $80 \%$ & $20 \%$ \\
Wellington Prison & 35 & $72 \%$ & $17 \%$ \\
Head Office & 18 & $28 \%$ \\
\hline
\end{tabular}

${ }^{\mathrm{a}}=$ These figures represent the total prison officer staff excluding instructors.

${ }^{186}$ Information for this table was sourced from an information sheet received from the Department of Corrections $5^{\text {th }}$ October 1998 and was noted as accurate as at 30 $0^{\text {th }}$ June 1998. 


\section{$\underline{\text { APPENDIX M }}$}

\section{EXAMPLES OF}

SHIFT ROUTINES ${ }^{187}$

${ }^{187}$ Information for this section derived from routine duty sheets sourced from each unit during the course of my research. 
ALPHA JAIL 


\section{ORANGE UNIT: DAILY ROUTINE}

0630 Unlock for cell cleaning/ cell inspection

$0730 \quad$ Breakfast

0930-0930 Gym orange north

or 1015-1115 Gym orange south

0845-1135 Yards

$1130 \quad$ Lunch

$1235 \quad$ Yards

1550 Yards in - lockup (mon-fri) for orange north

1630 Tea parade

1645 Lock up for orange south

1930 (mon-fri) General lockup (for those who have been here for 2 weeks or longer and are approved and are in orange north).

1950 (sat-sun) General lock up 


\section{ORANGE UNIT: 6 - 2 (S)}

0600 Briefed by Unit IC. Early breakfasts, court movements, observations.

0615 Commence unlocks orange south wing, kitchen workers, and segregated inmates (maximum two at a time).

Monitor kitchen and kitchen inmates randomly until 8.50am - ensure you muster them at least half hourly.

0655 All segregated inmates ablutions completed. Locked kitchen inmates to kitchen.

$0700 \quad$ 7-3S officer in wing. Feed segregation inmates.

0715 Orange inmates unlocked for cell cleaning, showers, etc. Cells open. Inmates may use phones, etc.

0730 Feed orange inmates in dining area only. Those inmates who do not qualify for various reasons to be fed in cells locked.

0800 Lock mainstream orange inmates. Unlock orange segregation. These are your messmen, wing cleaners, etc. If short of segregation workers lock middle grill - mainstream to clean mess, cells and corridor; segregation to clean showers, staff toilet, their corridors.

0850 Search and inspect yards - move to sentry.

0900 Sentry officer - maintain yard musters and inmate observations.

0945 Lunchbreak.

1015 Gym officer for south wing orange inmates assisted by 8-4A officer.

1115 Return inmates to yard NOT wing, yard two.

1120 Ensure any segregated inmates in yard one are sent back to wing. Can be locked by kitchen officer.

1130 Clear yard two of orange south wing inmates via kitchen.

Supervise orange south - allow telephone calls, etc. Ensure inmates leave tables and area clean and tidy.

1230 Yards run - as instructed by Unit IC.

1245 Stay in orange south.

1345 Cell searches, inmate movements, etc.

1400 Off shift. 


\section{ORANGE UNIT: 7 - 3(N)}

$0700 \quad$ Briefed by Unit IC. Early breakfasts, court movements, observations. Cell cleaning separates area with 6-2N officer. Assist morning routine orange north wing.

0730 Breakfast separates/ orange north wing. All inmates to eat in cells, only exceptions are workers as directed. Clear dishes from separates/ north wing. Continue with showers, cell cleaning if necessary.

0800 Assist with stripping inmates and escorts to receiving officer (both ends of unit).

0830 Gym for orange north wing inmates. 8-4A officer will assist.

0930 Inmates to return to wing locked or into yards.

0940 Sentry officer.

1130 Yards cleared. Move to orange north wing and assist with supervision of lunch mess/ wing area.

1200 Lunchbreak once relieved by 12-8 officers.

1230 To orange north, see IC and receive any updates on visits, etc. Move to visits area. Ensure visits area set out properly. Get start number of visitors sign in slips. Ensure all visitors let into area have a current visitors pass. At end of visits ensure all inmates searched and returned to proper yard or wing.

1500 Off shift.

\section{ORANGE UNIT: 7 - 3(S)}

0700 Briefed by Unit IC. Assist with morning routine orange south wing. Feed segregation inmates, then lock by 0715 hours.

0715 Orange inmates unlocked for cell cleaning, showers, etc. Cells open.

0730 Breakfast inmates.

0800 Lock orange south. Report to shift IC. Assist with movements to court (both wings). Report to IC and assist as directed by Unit IC.

0900 Medical for orange unit as required. Once finished inform Unit IC.

1120 Move to orange south wing. Ensure messman has set up for lunch. Any segregated inmates to be fed in cells are done so and locked.

1130 Orange inmates from yards/ wing, cells open. To be fed in mess area. Maintain supervision of wing/ mess area. This is the time for phone calls. Dishes cleared from locked inmates.

1200 Lunchbreak once relieved by 12-8 officers.

1230 To orange north, see IC and receive any updates or visits, etc. Move to visits area. Ensure visits are set out properly. Get start number of visitors sign in slips. Ensure all visitors let into area have a current visitors pass. At end of visits ensure all inmates searched and returned to proper yard or wing. Off shift. 


\section{ORANGE UNIT: 8 - 4 (A)}

0800 Briefed by Unit IC. Move to orange north wing. Assist with courts, etc. (both wings). Ensure all inmates going to the receiving officer are searched, including their belongings.

0830 Gym for orange north inmates (maximum 16). 7-3N will assist.

0930 Return inmates to yard 3 or wing and relieve IC for lunchbreak.

1015 Gym officer for south wing orange inmates assisted by 6-2S officer.

1115 Return inmates to yards, NOT wing, yard two. Assist 6-2N to feed separates. Remain in orange north wing.

1130 Ensure yard one and two have been cleared. Clear yard three to north wing. Assist with lunch parade orange north wing supervision of mess/ work area.

1200 Lunchbreak.

1230 Visits, you are in room opposite yellow unit guardroom. Ensure you are up to date with banned visitors, booth visits, etc., have the visitors passbook, both property books, money receipt book, etc. You sign in all visitors, receipt approved property and money. Only allow in approved property ie no red clothing, etc. Write the property up there and then in front of the visitor returning any property that is not allowed. Give the visitor the top copy when you have completed it. Periodically take the money received and place in yellow unit safe.

1445 On completion of visits take property to orange and issue to the inmates. DO NOT leave any property for anyone else to issue.

1530 Carry on in orange north.

1545 Take purple unit inmate workers back to purple unit.

1600 Off shift. 


\section{ORANGE UNIT: 9-6(B)}

\section{Monday and Friday}

0900 1) Monday, phone purple unit, confirm two purple unit inmates required for tip run 0900 hours.

2) Confirm with Unit Manager orange inmates to work on gang. Only Unit Manager has authority to assign inmates to gang. Unit Manager will brief you on any additional tasks required.

3) Locate truck, check that it is serviceable.

4) Go to purple unit and pick up two inmates assigned.

NOTE: If you do not have HT license, locate ay other officer in orange unit who does and he or she will do the tip run. You will stand in for that particular officer while they are away.

Tip run to be done twice per week, Monday and Friday am.

When returning from tip use purple unit inmates to unload truck. Return inmates to purple unit. Sign off truck.

\section{NOTE:}

1) Monday and Friday am if time warrants, you can start gang am after completion of tip run.

2) When the middle padlock gate is opened for any reason, ensure all orange inmates are in your sight at all times.

\section{Tuesday, Wednesday, Thursday}

0900 Start shift. Briefed by Unit IC. Assist where directed by Unit Manager.

Prior to 0930 conduct a security check of gang area. Account for all tools, etc. Remember these are orange inmates and you need to remain vigilant at all times.

0930 Commence gang responsibilities. Direct supervision of inmates in your care. At all times be able to account for each inmate.

1030 Smoko for inmates is 10 minutes. You can organise pot of tea from orange mess. Remember smoko is a privilege and may depend on gang's work output. Smoko is outside in work area.

1130 Gang to wing - account for all tools.

\section{LUNCHBREAK 1 hour}

1235 Resume gang responsibilities.

1530 Gang to wing. May shower before lockdown. Account for all tools. Check area for any security breach.

1600 Assist with tea parade and supervision of south wing with 2-10 and kitchen officer. Once relieved by 12-8S officer, move to north wing and assist.

1620 Supervise tea parade of orange south inmates with 2-10.

1640 Assist 12-8S. Lock orange south inmates, unlock segregated inmates for tea.

1710 All inmates locked except a maximum of $\underline{5}$ segregated inmates (kitchen workers and/ or laundry and/ or messman). Work as directed by IC. Musters, new arrivals, interviews, etc. 


\section{ORANGE UNIT: $12-8(\mathrm{~N})$}

1200 Briefed by Unit IC. Stay in orange north wing. Relieve officers for lunchbreak. Maintain supervision of orange inmates/ mess/ wing area.

1230 Yards or locked for orange inmates. Carry on with musters, cell searches, security checks, etc., and other duties as directed by IC.

1555 Yard into wing as instructed by Unit IC.

1600 Lunchbreak.

1630 Return to north wing. Assist with tea parade, supervision of mess/ wing area. Dishes cleared from inmates in wing who are locked. Musters. As directed by Unit IC.

1930 Lock up all inmates.

1935 Lock up muster accounted for. Paperwork, etc.

2000 Off shift.

\section{ORANGE UNIT: $12-8$ (S)}

1200 Briefed by Unit IC. Move to orange south wing. Relieve officers for lunchbreak.

1230 Sentry officer - maintain yard musters, inmate observations. Run yard one and two first then inform IC that yard three can be run.

1400 Relived by 2-10 officer. Move to south wing. Musters.

1530 Segregation workers to shower, if necessary. Locked by 1555.

1555 Orange inmates to wing from yard. Cells remain unlocked to 1640 for use of phone, etc. Supervised by 8-5 officer and 2-10 officer.

1600 Lunchbreak.

1630 Return to south wing. Assist with supervision mess/ wing area.

1640 South wing orange inmates locked. Segregated inmates on recreation and workers unlocked for tea and recreation. Maintain: supervision of wing; musters; new arrival interviews; as directed by Unit IC.

1710 Lockdown of all inmates except for a maximum of $\underline{5}$ segregated inmates consisting of the kitchen workers and/ or laundry and/ or messman.

1930 Lockup all remaining inmates.

1935 Lock up muster accounted for. Paperwork completed and to Unit IC.

2000 Off shift. 


\section{ORANGE UNIT: 2 - 10}

1400 Briefed by Unit IC. Move to sentry - maintain yard musters and inmate observations.

1600 Relieve 12-8S for their tea break. Supervision of south wing orange inmates assisted by 9-6B officer and kitchen officer.

1620 South wing orange inmates fed. Those inmates locked fed in cells.

1630 Once 12-8S back in remand south feed separates with kitchen instructor. Ensure rear doors in capital cells are locked.

1715 Tea break.

1745 Work as directed by IC. Supervision of inmates on recreation, musters, new arrival interviews, as directed by Unit IC.

1930 Lockup all inmates.

1935 Lockup muster accounted for. Maintain unit supervision and assist Unit IC.

2100 Move to yellow unit and conduct duties as per yellow unit orders.

2200 Off shift. 


\section{ORANGE UNIT: MEAL PARADES}

\section{BREAKFAST}

0730 Separates fed by 6-2N and 7-3N officers

South wing supervised by $6-2 \mathrm{~S}$ and 7-3S officers

North wing supervised by 6-2 IC, 6-2N and 7-3N officers

South wing segregated inmates fed in mess

Orange north inmates fed breakfast

Lock segregated inmates as soon as they are finished breakfast

Feed orange south inmates breakfast, then locked by 0800 hours.

\section{LUNCH}

1120 South wing segregated inmates fed in mess, then

North wing fed in mess, then

Lock or yards for segregated inmates as soon as they have finished lunch, then

Feed south wing orange inmates, then

Separates fed by 6-2N and kitchen instructor after everyone else fed.

TEA

1620 Feed south wing orange inmates supervised by 2-10 and 9-6B, kitchen officer.

1630 Separates fed by 2-10 and kitchen instructor when 12-8 officers get back. North wing inmates supervised by 1-9 IC, 12-8N, 2-10 officers, then

Orange south inmates locked at 1640 hours, then

Feed south wing segregated inmates after mainstream inmates locked.

NOTE: one officer to clear dishes from separates and orange north leaving the other officer to supervise wing and mess area.

\section{ORANGE UNIT: YARD CO-ORDINATION}

\section{AM YARDS}

0900 Orange south wing inmates YARD TWO

Segregation south wing inmates YARD ONE

Orange north wing inmates YARD THREE

1130 Segregation south wing inmates YARD ONE

Orange south wing inmates YARD TWO

Orange north wing inmates YARD THREE

\section{PM YARDS}

1230 Orange south wing inmates YARD TWO

Segregation south wing inmates YARD ONE

Orange north wing inmates YARD THREE

1555 Orange south wing inmates YARD ONE

Segregation south wing inmates YARD TWO

Orange north wing inmates YARD THREE

NOTE: A minimum of $25 \%$ of the inmates going to the yards are to be rubbed down every time the yards are run. 
Each Day is Different: Prison Officers and Their Work

BETA JAIL 


\section{GREY UNIT: DAILY ROUTINE (MONDAY-FRIDAY)}

6.00am Staff changeover/ staff to duty

6.05am Unlock kitchen inmates and others as required

6.30am General unlock: muster check

Those inmates reporting sick are to be identified and locked until seen by medical.

7.00am Breakfast parade

7.15am Muster check into dining room. All inmates must report to dining room before 7.15am.

7.45am Cell inspection: cells cleared for work parade - cell doors locked. Inmates not assigned to work or programme activities are to be locked in cells.

8.00am Staff changeover/ 8-4 staff to duty

8.05am Work parade

Inmates assigned daily duties. Work parties depart institution. General supervision directed by officer IC. Programme activities co-ordinated. Cell doors unlocked as required. Muster accounted for and recorded.

10.00am Unit smoko

10.15am Work resumes

11.30am Work parties return to institution

As required cells unlocked. Officer to check inmates in.

11.45am Lunch parade Muster check into dining room.

$12.30 \mathrm{pm}$ Work parade Inmates assigned daily duties continue. General supervision directed by officer. Programme activities co-ordinated.

$1.00 \mathrm{pm}$ Officer in charge of shift to duty

2.00pm Staff changeover (6-2 and 2-10)

2.30pm Unit smoko

$2.45 \mathrm{pm}$ Work resumes

$3.45 \mathrm{pm}$ Work parties return to institution

Cells unlocked. Officer to compound grille to muster in. Strip-searches at random. Muster check.

4.00pm Staff changeover (8-4)

$4.45 \mathrm{pm}$ Evening meal parade

Inmates muster check into dining room.

5.30pm Evening recreation commences

$6.00 \mathrm{pm}$ Organised evening activities commence

Outside groups, hobbies, education classes.

8.00pm Organised evening activities cease

Recreation rooms locked, programmes finished.

8.20pm General lock up call given

Personal admin before general lock down. Movement cease - all inmates to be in own cells.

8.30pm General lock up muster check

9.00pm Staff changeover (1-9, shift IC off duty)

$10.00 \mathrm{pm}$ Staff changeover

Muster checks are to be carried out at no longer than 60-minute intervals as per criteria set. 


\section{GREY UNIT: 6.00AM - 2.00PM}

6.00am Commence duty; sign time book; check for special instructions in log book; receive instructions from officer in charge of shift.

Early unlocks:

Early unlocks will be carried out at the appropriate times, as per daily unlock list. Inmates designated to work in the institution, eg kitchen, administration area, are to be unlocked and given appropriate time (15 minutes) before they are required in those areas. Inmates working in the kitchen area are to be checked on a regular basis.

6.15am Separates confinement requirements

Assist with removal of bedding and issue clothing for those inmates undergoing cell confinement.

6.30am General unlock of institution

Assist as required. Wings to be patrolled and officers are to ensure that inmates are awake and out of bed. Assist with the supervision of early inmates in kitchen area.

6.45am Separates breakfast

7.00am Breakfast parade

Supervise kitchen inmates on serving of breakfast. Assist with overall supervision of dining room. Oversee kitchen clean up activities, undertake duties as assigned by officer in charge.

7.45am Cell inspection

Ensure all inmates' cells are to standard. All inmates are to be properly dressed and shaved.

7.50am Assemble work parole

Assist in assembling work parade. Ensure all inmates are in correct work parties. Lock all inmates not involved in daily activities, eg unemployed etc.

8.00am Work parade

Assume control on inmates work parade. Maintain order until day staff arrive on parade.

8.05am Work parade dismissal

Once having been checked and any irregularities noted by officer in charge, assume control of all inmates who remain inside institution for the day. These inmates will be compound cleaner/s, groundsman, and those inmates who have been allocated an appointment time to attend their programme, and also those who have been locked down as sick.

8.10am Work allocation of inmates

Assign work to these inmates who have daily tasks, eg cleaner/s, groundsman. Co-ordinate all appointments of inmates with programmes party officer and ensure inmates are at their appointment on time. Ensure the institution has a high standard of cleanliness and undertake general duties as directed by the officer in charge, eg cell searches, security checks, etc.

11.00am General unlock

Unlock inmates in cells to continue daily routines.

11.30am Cell unlocked work parties return

Officer to assist officer in charge to muster in work parties (strip searches at random).

11.45am Lunch parade

Muster check into dining room. Inmates locked and/ or sick to be fed in cells. Supervise lunch parade and dining room.

\subsection{0pm Cells cleared - cell door locked}

$12.30 \mathrm{pm}$ Work parade

Work allocation of inmates continues. Programme activities co-ordinated. General supervision directed by officer in charge.

$1.50 \mathrm{pm}$ Complete daily marks sheet; relay any information to $2.00 \mathrm{pm}-10.00 \mathrm{pm}$ institution officer eg programmes and work activities of inmates. 


\section{GREY UNIT: 2.10PM (1) 2.10PM (2) - 10.00PM}

2.00pm Commence duty; sign time book; uplift keys; report to supervision officer

Receive any special instructions and be fully conversant with the state of the institution regarding covering all areas during the period prior to evening meal. Ensure all inmates are accounted for and the muster is correct. Ensure all inmates have changed into recreation clothing prior to evening meal parade.

4.30pm Separates evening meal

Feed separates in cells as per separates instructions.

\subsection{5pm Evening meal parade}

Supervise dining room area as per meal parade instructions. Muster check into dining room.

\section{$5.30 \mathrm{pm}$ Supervise evening recreation}

\section{$6.00 \mathrm{pm}$ Organised evening activities commence}

\subsection{0pm Mid evening patrols and musters}

Generally patrol the institution and assist as required, maintaining hourly muster checks ensuring these musters are logged. Report to the officer in charge anything of note. Ensure the compound is properly supervised at all times.

Assist with bedding of separates.

Prepare institution for lockup

Ensure areas being used by inmates during evening activities are cleaned eg TV rooms, classroom, poolroom etc.

Commence pegging patrols.

\subsection{0pm Lock up call given}

\subsection{0pm Commence general lock down}

Full visual muster check of all inmates to be undertaken. Check all padlocks and padbolts ensuring they are secure. Sign all lockup muster and search books.

\subsection{5pm Supervision patrol}

2-10 (1) Officer and officer in charge of 1-9 shift to complete a patrol, checking all doors, windows, and outer buildings are secure, all lights required for night supervision are working, and also complete a full peg round.

\section{2-10pm (1) Officer}

Ensure all keys are accounted for in guardroom key press.

Lockup books have been entered completely and accurately.

Muster is correct and signed for by two officers.

Assume control of institution from PM supervision officer

\section{Evening patrols}

At all times between the hours of general lock up, one shift officer, ie 2-10pm (1) 2-10pm (2) must patrol the institution continuously visiting all areas and peg as per pegging criteria at irregular intervals. Patrols should be carried out with a staff member in the guardroom area for support and to maintain radio and telephone communications.

Patrols are to include all institution areas and the separates. If inmates are confined in the separates, there are to be body checks carried out every hour. No inmates should be unlocked after 8.30pm and only in the direst of emergencies should this be considered and with at least two officers present.

\subsection{0pm 2-10pm (1) officer handover}

2-10pm (1) officer to do complete check of institution and ensure all is in order prior to handing over to NW (1). Check all keys. Leave any special instructions.

$10.00 \mathrm{pm}$ Sign time book; cease duty. 


\section{$\underline{\text { APPENDIX N }}$}

\section{TARGET PRISONS' \\ CLIQUES’ AND GROUPS' DEMOGRAPHY}




\section{Table N.1}

Demographic Structure of the New Zealand Prison Officer Cliques and Groups From the Target Institution

\begin{tabular}{|c|c|c|c|c|c|c|c|c|c|c|c|c|c|c|c|c|c|c|c|c|c|}
\hline \multicolumn{2}{|c|}{ DEMOGRAPHIC CATEGORY } & \multicolumn{20}{|c|}{ CLIQUE/ GROUP CATEGORIES (N=114 - Questionnaire respondents only) } \\
\hline & & \multicolumn{2}{|c|}{$\begin{array}{l}\text { SA'AS } \\
(\mathrm{N}=9)\end{array}$} & \multicolumn{2}{|c|}{$\begin{array}{c}\text { MAORI } \\
\text { UNSPECIFIED } \\
(\mathbf{N}=\mathbf{1 4})\end{array}$} & \multicolumn{2}{|c|}{$\begin{array}{c}\text { BROS } \\
(\mathrm{N}=14)\end{array}$} & \multicolumn{2}{|c|}{$\begin{array}{c}\text { BLACK } \\
\text { MAFIA } \\
(\mathrm{N}=3)\end{array}$} & \multicolumn{2}{|c|}{$\begin{array}{c}\text { MoLL } \\
\text { PATROL } \\
(\mathbf{N}=4)\end{array}$} & \multicolumn{2}{|c|}{$\begin{array}{c}\text { SENIOR - } \\
\text { THREE BARS } \\
(\mathbf{N = 1 0 )}\end{array}$} & \multicolumn{2}{|c|}{$\begin{array}{c}\text { SENIOR - } \\
\text { TWO BARS } \\
(\mathbf{N}=\mathbf{2 0})\end{array}$} & \multicolumn{2}{|c|}{$\begin{array}{l}\text { FUNCTIONARIES } \\
(\mathbf{N}=\mathbf{2 9})\end{array}$} & \multicolumn{2}{|c|}{$\begin{array}{c}\text { BURNOUTS } \\
(\mathrm{N}=2)\end{array}$} & \multicolumn{2}{|c|}{$\begin{array}{c}\text { PAKEHA } \\
\text { WOMEN } \\
(\mathrm{N}=9)\end{array}$} \\
\hline \multirow[t]{2}{*}{ GENDER } & Male & (7) & $77.8 \%$ & (12) & $85.7 \%$ & (14) & $100 \%$ & (3) & $100 \%$ & $(0)$ & $0.0 \%$ & (9) & $90.0 \%$ & (20) & $100 \%$ & (29) & $100 \%$ & (2) & $100 \%$ & $(0)$ & $0.0 \%$ \\
\hline & Female & (2) & $22.2 \%$ & (2) & $14.3 \%$ & $(0)$ & $0.0 \%$ & (0) & $0.0 \%$ & (4) & $100 \%$ & (1) & $10.0 \%$ & $(0)$ & $0.0 \%$ & (0) & $0.0 \%$ & (0) & $0.0 \%$ & (9) & $100 \%$ \\
\hline \multirow[t]{3}{*}{ ETHNICITY } & Pakeha & $(0)$ & $0.0 \%$ & $(0)$ & $0.0 \%$ & $(2)$ & $14.3 \%$ & (1) & $33.3 \%$ & $(0)$ & $0.0 \%$ & (9) & $90.0 \%$ & (18) & $90.0 \%$ & (29) & $100 \%$ & (2) & $100 \%$ & (9) & $100 \%$ \\
\hline & Maori & (0) & $0.0 \%$ & (14) & $100 \%$ & (10) & $71.4 \%$ & (2) & $66.7 \%$ & (4) & $100 \%$ & (1) & $10.0 \%$ & (2) & $10.0 \%$ & $(0)$ & $0.0 \%$ & (0) & $0.0 \%$ & (0) & $0.0 \%$ \\
\hline & Pacific Island & (9) & $100 \%$ & $(0)$ & $0.0 \%$ & (2) & $14.3 \%$ & (0) & $0.0 \%$ & (0) & $0.0 \%$ & (0) & $0.0 \%$ & (0) & $0.0 \%$ & (0) & $0.0 \%$ & (0) & $0.0 \%$ & (0) & $0.0 \%$ \\
\hline SERVICE & $0-4$ years & (3) & $33.3 \%$ & $(10)$ & $71.4 \%$ & (3) & $21.4 \%$ & (1) & $33.3 \%$ & $(0)$ & $0.0 \%$ & (0) & $0.0 \%$ & (2) & $10.0 \%$ & (26) & $89.7 \%$ & (1) & $50.0 \%$ & (6) & $66.7 \%$ \\
\hline \multirow[t]{2}{*}{ LENGTH } & 4-10 years & (3) & $33.3 \%$ & (3) & $21.4 \%$ & (5) & $35.7 \%$ & (2) & $66.7 \%$ & (4) & $100 \%$ & (0) & $0.0 \%$ & (18) & $90.0 \%$ & (3) & $10.3 \%$ & (1) & $50.0 \%$ & (3) & $33.3 \%$ \\
\hline & $10+$ years & (3) & $33.3 \%$ & (1) & $7.1 \%$ & (6) & $42.9 \%$ & $(0)$ & $0.0 \%$ & $(0)$ & $0.0 \%$ & (10) & $100 \%$ & $(0)$ & $0.0 \%$ & (0) & $0.0 \%$ & (0) & $0.0 \%$ & (0) & $0.0 \%$ \\
\hline \multirow[t]{5}{*}{ JOB TITLE } & Probationary Officer & $(0)$ & $0.0 \%$ & (3) & $21.4 \%$ & $(0)$ & $0.0 \%$ & $(0)$ & $0.0 \%$ & $(0)$ & $0.0 \%$ & $(0)$ & $0.0 \%$ & $(0)$ & $0.0 \%$ & $(17)$ & $58.6 \%$ & $(0)$ & $0.0 \%$ & (4) & $44.4 \%$ \\
\hline & Prison Officer & (4) & $44.4 \%$ & (6) & $42.9 \%$ & (6) & $42.9 \%$ & (1) & $33.3 \%$ & (3) & $75.0 \%$ & (1) & $10.0 \%$ & (7) & $45.0 \%$ & (8) & $27.6 \%$ & (1) & $50.0 \%$ & (3) & $33.3 \%$ \\
\hline & Instructor Officer & (1) & $11.1 \%$ & (3) & $21.4 \%$ & (1) & $7.1 \%$ & (1) & $33.3 \%$ & (0) & $0.0 \%$ & (1) & $10.0 \%$ & (5) & $25.0 \%$ & (3) & $10.3 \%$ & (1) & $50.0 \%$ & (0) & $0.0 \%$ \\
\hline & Unit IC & (4) & $44.4 \%$ & (1) & $7.1 \%$ & (6) & $42.9 \%$ & (1) & $33.3 \%$ & (1) & $25.0 \%$ & (6) & $60.0 \%$ & (7) & $45.0 \%$ & (1) & $3.4 \%$ & (0) & $0.0 \%$ & (1) & $11.1 \%$ \\
\hline & Unit UM & $(0)$ & $0.0 \%$ & (1) & $7.1 \%$ & (1) & $7.1 \%$ & (0) & $0.0 \%$ & $(0)$ & $0.0 \%$ & (2) & $20.0 \%$ & (1) & $5.0 \%$ & $(0)$ & $0.0 \%$ & (0) & $0.0 \%$ & (1) & $11.1 \%$ \\
\hline MilitaRY & Military Experience & (1) & $11.1 \%$ & (4) & $25.6 \%$ & $(2)$ & $14.3 \%$ & (3) & $100 \%$ & (1) & $25.0 \%$ & (4) & $40.0 \%$ & (4) & $20.0 \%$ & $(16)$ & $55.2 \%$ & $(0)$ & $0.0 \%$ & (1) & $11.1 \%$ \\
\hline EXPERIENCE & No Military Experience & (7) & $77.8 \%$ & (10) & $71.4 \%$ & $(12)$ & $85.7 \%$ & $(0)$ & $0.0 \%$ & (3) & $75.0 \%$ & (6) & $60.0 \%$ & (16) & $80.0 \%$ & (13) & $44.8 \%$ & (2) & $100 \%$ & (8) & $88.9 \%$ \\
\hline AdDitional & No Experience & (5) & $55.6 \%$ & $(12)$ & $85.7 \%$ & (9) & $64.3 \%$ & (3) & $100 \%$ & $(2)$ & $50.0 \%$ & (1) & $10.0 \%$ & $(12)$ & $60.0 \%$ & (28) & $96.6 \%$ & $(0)$ & $0.0 \%$ & (6) & $66.7 \%$ \\
\hline JAIL & Additional Experience & (3) & $33.3 \%$ & (2) & $14.3 \%$ & (4) & $25.6 \%$ & $(0)$ & $0.0 \%$ & (2) & $50.0 \%$ & (9) & $90.0 \%$ & (8) & $40.0 \%$ & (1) & $3.4 \%$ & (2) & $100 \%$ & (3) & $33.3 \%$ \\
\hline
\end{tabular}

Note. Percentages rounded, totals may not equal $100 \%$. 


\section{$\underline{\text { APPENDIX } 0}$}

\section{SUPPLEMENTARY}

DEMOGRAPHIC

TABLES FOR

MotivaTiONS FoR

BECOMING A PRISON

OFFICER 
Table 0.1

Motivations for Becoming a Prison Officer by Jail Type at the Target Institution

\begin{tabular}{|c|c|c|c|c|c|}
\hline \multirow{2}{*}{$\begin{array}{l}\text { Alpha Jail Hierarchy of Motivations } \\
\text { For Becoming a Prison Officer } \\
\text { "The job looked interesting” }\end{array}$} & \multicolumn{2}{|c|}{$\begin{array}{l}\text { Alpha Officers } \\
\qquad(\mathrm{N}=45)\end{array}$} & \multirow{2}{*}{$\begin{array}{l}\text { Beta Jail Hierarchy of Motivations } \\
\text { For Becoming a Prison Officer }\end{array}$} & \multicolumn{2}{|c|}{$\begin{array}{c}\text { Beta Officers } \\
(\mathrm{N}=70)\end{array}$} \\
\hline & (23) & $51.1 \%$ & & (43) & $61.4 \%$ \\
\hline “The challenge/ excitement” & (21) & $46.7 \%$ & “The challenge/ excitement” & (39) & $55.7 \%$ \\
\hline "For the money" & (17) & $37.8 \%$ & “For the money” & (29) & $41.4 \%$ \\
\hline “Just needed a job” & (13) & $28.9 \%$ & “Just needed a job” & (18) & $25.7 \%$ \\
\hline “To help reduce re-offending” & (11) & $24.4 \%$ & “To help reduce re-offending” & (13) & $18.6 \%$ \\
\hline "Friends/ relatives were prison officers" & (9) & $20.0 \%$ & "Friends/ relatives were prison officers" & (13) & $18.6 \%$ \\
\hline Job security & $(2)$ & $4.4 \%$ & “Always wanted to be a prison officer” & (6) & $8.6 \%$ \\
\hline Enjoy working with people & (2) & $4.4 \%$ & “Other” & (6) & $8.6 \%$ \\
\hline “Always wanted to be a prison officer" & $(2)$ & $4.4 \%$ & Job security & (4) & $5.7 \%$ \\
\hline "Power" & (1) & $2.2 \%$ & Enjoy working with people & (2) & $2.9 \%$ \\
\hline “Other” & $(0)$ & $0.0 \%$ & "Power” & (1) & $1.4 \%$ \\
\hline
\end{tabular}

Note. Multiple responses permitted therefore totals do not equal 100\%. Dotted line indicates the division for Expected N, categories above have positive residuals, categories below have negative residuals. 
Table 0.2

Motivations for Becoming a Prison Officer by Gender at the Target Institution

\begin{tabular}{|c|c|c|c|c|c|}
\hline $\begin{array}{l}\text { Male Hierarchy of Motivations For } \\
\text { Becoming a Prison Officer }\end{array}$ & \multicolumn{2}{|c|}{$\begin{array}{l}\text { Male Officers } \\
\quad(\mathrm{N}=97)\end{array}$} & $\begin{array}{l}\text { Female Hierarchy of Motivations } \\
\text { For Becoming a Prison Officer }\end{array}$ & \multicolumn{2}{|c|}{$\begin{array}{c}\text { Female } \\
\text { Officers }(\mathrm{N}=18)\end{array}$} \\
\hline "The job looked interesting" & (56) & $57.7 \%$ & "For the money" & $(12)$ & $66.7 \%$ \\
\hline "The challenge/ excitement" & (48) & $49.5 \%$ & "The challenge/ excitement" & (12) & $66.7 \%$ \\
\hline "For the money" & (34) & $35.1 \%$ & "The job looked interesting” & (10) & $55.6 \%$ \\
\hline "Just needed a job" & (25) & $25.8 \%$ & “To help reduce re-offending” & (7) & $38.9 \%$ \\
\hline "Friends/ relatives were prison officers" & (21) & $21.6 \%$ & “Just needed a job” & (6) & $33.3 \%$ \\
\hline “To help reduce re-offending” & (17) & $17.5 \%$ & "Always wanted to be a prison officer" & (4) & $22.2 \%$ \\
\hline Job security & (6) & $6.2 \%$ & Enjoy working with people & (2) & $11.1 \%$ \\
\hline "Other" & (6) & $6.2 \%$ & "Friends/ relatives were prison officers" & (1) & $5.6 \%$ \\
\hline "Always wanted to be a prison officer" & (4) & $4.1 \%$ & “Power” & (0) & $0.0 \%$ \\
\hline "Power" & (2) & $2.1 \%$ & Job security & (0) & $0.0 \%$ \\
\hline Enjoy working with people & (2) & $2.1 \%$ & "Other" & (0) & $0.0 \%$ \\
\hline
\end{tabular}

Note. Multiple responses permitted therefore totals do not equal 100\%. Dotted line indicates the division for Expected N, categories above have positive residuals, categories below have negative residuals. 


\section{Table 0.3}

Motivations for Becoming a Prison Officer by Ethnicity of Officers at the Target Institution

\begin{tabular}{|c|c|c|c|c|c|c|c|c|}
\hline \multirow{2}{*}{$\begin{array}{l}\text { Pakeha Hierarchy of Motivations For } \\
\text { Becoming a Prison Officer }\end{array}$} & \multicolumn{2}{|c|}{$\begin{array}{l}\text { Pakeha } \\
\text { Officers } \\
(\mathrm{N}=72)\end{array}$} & \multirow{2}{*}{$\begin{array}{l}\text { Maori Hierarchy of Motivations For } \\
\text { Becoming a Prison Officer }\end{array}$} & \multicolumn{2}{|c|}{$\begin{array}{c}\text { Maori } \\
\text { Officers } \\
(\mathrm{N}=32)\end{array}$} & \multirow{2}{*}{$\begin{array}{c}\text { Pacific Island Hierarchy of } \\
\text { Motivations For Becoming a Prison } \\
\text { Officer }\end{array}$} & \multicolumn{2}{|c|}{$\begin{array}{c}\text { Pacific Island } \\
\text { Officers } \\
(\mathrm{N}=11)\end{array}$} \\
\hline & $(43)$ & $59.7 \%$ & & (25) & $78.1 \%$ & & (7) & $63.6 \%$ \\
\hline “The challenge/ excitement” & (34) & $47.2 \%$ & “The job looked interesting” & $(23)$ & $71.9 \%$ & “The challenge/ excitement” & (7) & $63.6 \%$ \\
\hline "For the money" & $(24)$ & $33.3 \%$ & "For the money" & (23) & $71.9 \%$ & "For the money" & (6) & $54.5 \%$ \\
\hline “To help reduce re-offending” & $(18)$ & $25.0 \%$ & "Just needed a job” & $(15)$ & $46.9 \%$ & “To help reduce re-offending” & $(4)$ & $36.4 \%$ \\
\hline “Just needed a job” & $(17)$ & $23.6 \%$ & "Friends/ relatives were prison officers" & $(10)$ & $31.3 \%$ & "Always wanted to be a prison officer" & (2) & $18.2 \%$ \\
\hline "Friends/ relatives were prison officers" & (11) & $15.3 \%$ & "Always wanted to be a prison officer" & (4) & $12.5 \%$ & "Friends/ relatives were prison officers" & (1) & $9.1 \%$ \\
\hline Job security & (4) & $5.6 \%$ & “To help reduce re-offending” & (4) & $12.5 \%$ & “Just needed a job” & (1) & $9.1 \%$ \\
\hline Enjoy working with people & (3) & $4.2 \%$ & “Other” & $(2)$ & $6.3 \%$ & "Other" & (1) & $9.1 \%$ \\
\hline “Always wanted to be a prison officer” & (3) & $4.2 \%$ & Job security & $(2)$ & $6.3 \%$ & "Power" & $(0)$ & $0.0 \%$ \\
\hline “Other” & (3) & $4.2 \%$ & “Power” & (1) & $3.1 \%$ & Job security & $(0)$ & $0.0 \%$ \\
\hline "Power" & $(1)$ & $1.4 \%$ & Enjoy working with people & $(1)$ & $3.1 \%$ & Enjoy working with people & $(0)$ & $0.0 \%$ \\
\hline
\end{tabular}

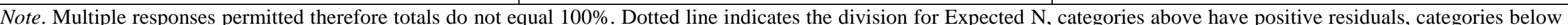
have negative residuals. Double dotted line indicates the enclosed variable was on the Expected $\mathrm{N}$ (hence neither a positive nor negative residual value). 


\section{Table 0.4}

Motivations for Becoming a Prison Officer by Service Length of Officers at the Target Institution

\begin{tabular}{|c|c|c|c|c|c|c|c|c|}
\hline $\begin{array}{l}\text { 0-4 years Hierarchy of Motivations } \\
\text { For Becoming a Prison Officer }\end{array}$ & \multicolumn{2}{|c|}{$\begin{array}{l}0-4 \text { Year } \\
(\mathrm{N}=53)\end{array}$} & $\begin{array}{l}\text { 4-10 years Hierarchy of Motivations } \\
\text { For Becoming a Prison Officer }\end{array}$ & \multicolumn{2}{|c|}{$\begin{array}{l}\text { 4-10 years } \\
(\mathrm{N}=42)\end{array}$} & $\begin{array}{c}\text { 10+ years Hierarchy of Motivations } \\
\text { For Becoming a Prison Officer }\end{array}$ & \multicolumn{2}{|c|}{$\begin{array}{l}\text { 10+ years } \\
(\mathrm{N}=20)\end{array}$} \\
\hline “The job looked interesting” & (35) & $66.0 \%$ & "The job looked interesting" & (25) & $59.5 \%$ & "Just needed a job" & $(11)$ & $55.0 \%$ \\
\hline "The challenge/ excitement” & $(27)$ & $50.9 \%$ & “The challenge/ excitement” & $(23)$ & $54.8 \%$ & "The challenge/ excitement" & (10) & $50.0 \%$ \\
\hline "For the money" & $(21)$ & $39.6 \%$ & "For the money" & $(16)$ & $38.1 \%$ & "For the money" & (9) & $45.0 \%$ \\
\hline “To help reduce re-offending” & (13) & $24.5 \%$ & “Just needed a job” & $(14)$ & $33.3 \%$ & “The job looked interesting” & (6) & $30.0 \%$ \\
\hline "Friends/ relatives were prison officers" & (9) & $17.0 \%$ & “To help reduce re-offending” & (8) & $19.0 \%$ & "Friends/ relatives were prison officers" & (6) & $30.0 \%$ \\
\hline "Just needed a job” & (6) & $11.3 \%$ & "Friends/ relatives were prison officers" & (7) & $16.7 \%$ & "To help reduce re-offending” & (3) & $15.0 \%$ \\
\hline “Always wanted to be a prison officer” & (4) & $7.5 \%$ & "Always wanted to be a prison officer" & (4) & $9.5 \%$ & Enjoy working with people & (1) & $5.0 \%$ \\
\hline “Other” & (3) & $5.7 \%$ & “Other” & (3) & $7.1 \%$ & "Power" & $(0)$ & $0.0 \%$ \\
\hline Job security & (3) & $5.7 \%$ & Job security & (3) & $7.1 \%$ & “Always wanted to be a prison officer” & $(0)$ & $0.0 \%$ \\
\hline Enjoy working with people & $(2)$ & $3.8 \%$ & "Power” & $(1)$ & $2.4 \%$ & Job security & $(0)$ & $0.0 \%$ \\
\hline "Power" & $(1)$ & $1.9 \%$ & Enjoy working with people & (1) & $2.4 \%$ & “Other” & (0) & $0.0 \%$ \\
\hline
\end{tabular}

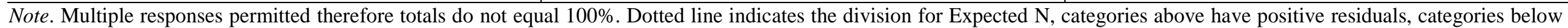
have negative residuals. 


\section{Table 0.5}

Motivations for Becoming a Prison Officer by Education Level of Officers at the Target Institution

\begin{tabular}{|c|c|c|c|c|c|c|c|c|}
\hline $\begin{array}{c}\text { No Education Level Officers' } \\
\text { Hierarchy of Motivations For Joining }\end{array}$ & \multicolumn{2}{|c|}{ None $(\mathrm{N}=17)$} & \multirow{2}{*}{$\begin{array}{c}\text { Low Secondary Level Education } \\
\text { Officers' Hierarchy of Motivations } \\
\text { For Joining }\end{array}$} & \multicolumn{2}{|c|}{$\begin{array}{c}\text { Low Secondary } \\
\text { Level }(\mathrm{N}=29)\end{array}$} & \multirow{2}{*}{$\begin{array}{c}\begin{array}{c}\text { High Secondary Level Education } \\
\text { Officers' Hierarchy of Motivations } \\
\text { For Joining }\end{array} \\
\text { “The challenge/ excitement” }\end{array}$} & \multicolumn{2}{|c|}{$\begin{array}{c}\text { High } \\
\text { Secondary } \\
\text { Level }(\mathrm{N}=15)\end{array}$} \\
\hline “The challenge/ excitement” & (10) & $58.8 \%$ & & (19) & $65.5 \%$ & & $(8)$ & $53.3 \%$ \\
\hline "The job looked interesting” & (7) & $41.2 \%$ & "The challenge/ excitement" & (18) & $62.1 \%$ & "For the money" & (7) & $46.7 \%$ \\
\hline "Friends/ relatives were prison officers" & (7) & $41.2 \%$ & "For the money" & (11) & $37.9 \%$ & “To help reduce re-offending” & (7) & $46.7 \%$ \\
\hline "For the money" & (7) & $41.2 \%$ & “Just needed a job” & (9) & $31.0 \%$ & “The job looked interesting” & $(6)$ & $40.0 \%$ \\
\hline "Just needed a job” & (3) & $17.6 \%$ & "Friends/ relatives were prison officers" & (5) & $17.2 \%$ & “Just needed a job” & (6) & $40.0 \%$ \\
\hline "Always wanted to be a prison officer" & $(2)$ & $11.8 \%$ & “To help reduce re-offending” & (3) & $10.3 \%$ & "Friends/ relatives were prison officers" & (3) & $20.0 \%$ \\
\hline “To help reduce re-offending” & (1) & $5.9 \%$ & "Always wanted to be a prison officer" & (2) & $6.9 \%$ & "Always wanted to be a prison officer" & (2) & $13.3 \%$ \\
\hline "Power" & $(0)$ & $0.0 \%$ & Job security & (1) & $3.4 \%$ & "Power” & (2) & $13.3 \%$ \\
\hline Job security & $(0)$ & $0.0 \%$ & Enjoy working with people & (1) & $3.4 \%$ & “Other" & $(2)$ & $13.3 \%$ \\
\hline Enjoy working with people & $(0)$ & $0.0 \%$ & "Power" & (0) & $0.0 \%$ & Job security & (1) & $6.7 \%$ \\
\hline “Other” & $(0)$ & $0.0 \%$ & “Other” & $(0)$ & $0.0 \%$ & Enjoy working with people & $(0)$ & $0.0 \%$ \\
\hline
\end{tabular}


Cont...

\begin{tabular}{|c|c|c|c|c|c|}
\hline $\begin{array}{c}\text { Low Tertiary Level Education } \\
\text { Officers' Hierarchy of Motivations } \\
\text { For Joining }\end{array}$ & $\begin{array}{l}\text { Low T } \\
\text { Level }\end{array}$ & $\begin{array}{l}\text { rrtiary } \\
\mathbf{N}=49 \text { ) }\end{array}$ & $\begin{array}{c}\text { High Tertiary Level Education } \\
\text { Officers' Hierarchy of Motivations } \\
\text { For Joining }\end{array}$ & $\begin{array}{c}\text { High } \\
\text { Leve }\end{array}$ & $\begin{array}{l}\text { ertiary } \\
(\mathrm{N}=5)\end{array}$ \\
\hline "The job looked interesting" & (32) & $65.3 \%$ & "The job looked interesting" & (2) & $40.0 \%$ \\
\hline "The challenge/ excitement" & (22) & $44.9 \%$ & "For the money" & (2) & $40.0 \%$ \\
\hline "For the money" & (19) & $38.8 \%$ & "The challenge/ excitement" & (2) & $40.0 \%$ \\
\hline "To help reduce re-offending" & (12) & $24.5 \%$ & “Just needed a job” & (1) & $20.0 \%$ \\
\hline “Just needed a job” & (12) & $24.5 \%$ & "To help reduce re-offending” & (1) & $20.0 \%$ \\
\hline "Friends/ relatives were prison officers" & (7) & $14.3 \%$ & "Friends/ relatives were prison officers" & (0) & $0.0 \%$ \\
\hline "Other” & (4) & $8.2 \%$ & Enjoy working with people & (0) & $0.0 \%$ \\
\hline Job security & (4) & $8.2 \%$ & Job security & (0) & $0.0 \%$ \\
\hline Enjoy working with people & (3) & $6.1 \%$ & "Always wanted to be a prison officer" & (0) & $0.0 \%$ \\
\hline "Always wanted to be a prison officer" & (2) & $4.1 \%$ & "Power” & (0) & $0.0 \%$ \\
\hline "Power" & (0) & $0.0 \%$ & "Other" & (0) & $0.0 \%$ \\
\hline
\end{tabular}

Note. Multiple responses permitted therefore totals do not equal $100 \%$. Dotted line indicates the division for Expected $\mathrm{N}$, categories above have positive residuals, categories below have negative residuals. 


\section{Table 0.6}

Motivations for Becoming a Prison Officer by Job Title of Officers at the Target Institution

\begin{tabular}{|c|c|c|c|c|c|c|c|c|}
\hline \multirow{2}{*}{$\begin{array}{l}\text { Probationary Officers' Hierarchy of } \\
\text { Motivations For Joining } \\
\text { “The job looked interesting” }\end{array}$} & \multicolumn{2}{|c|}{$\begin{array}{c}\text { Probationary } \\
\text { Officer } \\
(\mathrm{N}=25)\end{array}$} & \multirow{2}{*}{$\begin{array}{l}\text { Prison Officers' Hierarchy of } \\
\text { Motivations For Joining }\end{array}$} & \multicolumn{2}{|c|}{$\begin{array}{l}\text { Prison Officer } \\
(\mathrm{N}=39)\end{array}$} & \multirow{3}{*}{$\begin{array}{l}\text { Instructor Officers' Hierarchy of } \\
\text { Motivations For Joining }\end{array}$} & \multicolumn{2}{|c|}{$\begin{array}{c}\text { Instructor } \\
\text { Officer } \\
(\mathrm{N}=16)\end{array}$} \\
\hline & (15) & $60.0 \%$ & & $(22)$ & $56.4 \%$ & & (13) & $81.3 \%$ \\
\hline “The challenge/ excitement” & (15) & $60.0 \%$ & “The challenge/ excitement” & $(17)$ & $43.6 \%$ & & $(10)$ & $62.5 \%$ \\
\hline "For the money" & $(10)$ & $40.0 \%$ & "For the money" & (15) & $38.5 \%$ & "Friends/ relatives were prison officers" & (4) & $25.0 \%$ \\
\hline “To help reduce re-offending” & (6) & $24.0 \%$ & “Just needed a job” & $(10)$ & $25.6 \%$ & “To help reduce re-offending” & (3) & $18.8 \%$ \\
\hline "Friends/ relatives were prison officers" & (3) & $12.0 \%$ & “To help reduce re-offending” & (9) & $23.1 \%$ & "For the money" & (3) & $18.8 \%$ \\
\hline “Just needed a job” & (3) & $12.0 \%$ & "Friends/ relatives were prison officers" & (6) & $15.4 \%$ & “Other” & (2) & $12.5 \%$ \\
\hline “Always wanted to be a prison officer” & (2) & $8.0 \%$ & "Always wanted to be a prison officer" & (3) & $7.7 \%$ & “Just needed a job” & (1) & $6.3 \%$ \\
\hline Job security & $(2)$ & $8.0 \%$ & "Power" & (3) & $7.7 \%$ & Job security & (1) & $6.3 \%$ \\
\hline Enjoy working with people & (1) & $4.0 \%$ & Job security & $(2)$ & $5.1 \%$ & Enjoy working with people & (0) & $0.0 \%$ \\
\hline "Power” & (0) & $0.0 \%$ & Enjoy working with people & $(2)$ & $5.1 \%$ & "Power" & $(0)$ & $0.0 \%$ \\
\hline "Other” & $(0)$ & $0.0 \%$ & “Other” & $(0)$ & $0.0 \%$ & “Always wanted to be a prison officer” & (0) & $0.0 \%$ \\
\hline
\end{tabular}




\section{Cont...}

\begin{tabular}{|c|c|c|c|c|c|}
\hline $\begin{array}{c}\text { Unit ICs' Hierarchy of Motivations } \\
\text { For Joining }\end{array}$ & Unit IC & $(\mathrm{N}=\mathbf{2 8})$ & $\begin{array}{l}\text { Unit Managers' Hierarchy of } \\
\text { Motivations For Joining }\end{array}$ & Unit & $\begin{array}{l}\text { lanager } \\
=6)\end{array}$ \\
\hline "For the money" & (16) & $57.1 \%$ & “The job looked interesting” & (5) & $83.3 \%$ \\
\hline "Just needed a job" & $(15)$ & $53.6 \%$ & "The challenge/ excitement” & (4) & $66.7 \%$ \\
\hline "The challenge/ excitement” & (14) & $50.0 \%$ & "Friends/ relatives were prison officers" & (2) & $33.3 \%$ \\
\hline "The job looked interesting” & $(10)$ & $35.7 \%$ & "To help reduce re-offending” & (2) & $33.3 \%$ \\
\hline "Friends/ relatives were prison officers" & (7) & $25.0 \%$ & "For the money" & (1) & $16.7 \%$ \\
\hline "To help reduce re-offending” & (4) & $14.3 \%$ & "Always wanted to be a prison officer" & (1) & $16.7 \%$ \\
\hline "Always wanted to be a prison officer" & $(2)$ & $7.1 \%$ & "Just needed a job" & (1) & $16.7 \%$ \\
\hline "Power" & $(2)$ & $7.1 \%$ & Enjoy working with people & (1) & $16.7 \%$ \\
\hline Job security & (1) & $3.6 \%$ & Job security & $(0)$ & $0.0 \%$ \\
\hline Enjoy working with people & (1) & $0.0 \%$ & "Power" & (0) & $0.0 \%$ \\
\hline “Other" & $(0)$ & $3.6 \%$ & “Other” & $(0)$ & $0.0 \%$ \\
\hline
\end{tabular}

Note. Multiple responses permitted therefore totals do not equal 100\%. Dotted line indicates the division for Expected $\mathrm{N}$, categories above have positive residuals, categories below have negative residuals. 


\section{Table 0.7}

Motivations for Becoming a Prison Officer by Military Experience of Officers at the Target Institution

\begin{tabular}{|c|c|c|c|c|c|}
\hline \multirow{2}{*}{$\begin{array}{l}\text { Previous Military Experience Officers' } \\
\text { Hierarchy of Motivations For Joining } \\
\text { “The job looked interesting” }\end{array}$} & \multicolumn{2}{|c|}{$\begin{array}{c}\text { Previous Military } \\
\text { Experience }(\mathrm{N}=37)\end{array}$} & \multirow{2}{*}{$\begin{array}{l}\text { No Previous Military Experience Officers' } \\
\text { Hierarchy of Motivations For Joining }\end{array}$} & \multicolumn{2}{|c|}{$\begin{array}{c}\text { No Previous } \\
\text { Military Experience } \\
(\mathrm{N}=77)\end{array}$} \\
\hline & (23) & $62.2 \%$ & & $(45)$ & $58.4 \%$ \\
\hline "The challenge/ excitement" & (15) & $40.5 \%$ & “The job looked interesting” & (43) & $55.8 \%$ \\
\hline "For the money" & $(12)$ & $32.4 \%$ & "For the money" & (34) & $44.2 \%$ \\
\hline “Just needed a job” & $(10)$ & $27.0 \%$ & "Just needed a job” & $(21)$ & $27.3 \%$ \\
\hline “To help reduce re-offending” & (8) & $21.6 \%$ & "Friends/ relatives were prison officers" & (18) & $23.4 \%$ \\
\hline "Friends/ relatives were prison officers" & (4) & $10.8 \%$ & “To help reduce re-offending" & (16) & $20.8 \%$ \\
\hline “Other” & (3) & $8.1 \%$ & "Always wanted to be a prison officer" & (6) & $7.8 \%$ \\
\hline "Always wanted to be a prison officer" & (2) & $5.4 \%$ & Job security & (5) & $6.5 \%$ \\
\hline Enjoy working with people & (1) & $2.7 \%$ & Enjoy working with people & (3) & $3.9 \%$ \\
\hline Job security & (1) & $2.7 \%$ & “Other” & (3) & $3.9 \%$ \\
\hline "Power" & (1) & $2.7 \%$ & "Power” & (1) & $1.3 \%$ \\
\hline
\end{tabular}

Note. Multiple responses permitted therefore totals do not equal 100\%. Dotted line indicates the division for Expected N, categories above have positive residuals, categories below have negative residuals. 


\section{Table 0.8}

Motivations for Becoming a Prison Officer by Additional Jail Experience of Officers at the Target Institution

\begin{tabular}{|c|c|c|c|c|c|}
\hline \multirow{2}{*}{$\begin{array}{c}\text { No Previous Additional Jail } \\
\text { Experience Officers' Hierarchy of } \\
\text { Motivations For Joining }\end{array}$} & \multicolumn{2}{|c|}{$\begin{array}{c}\text { No Previous } \\
\text { Additional Jail } \\
\text { Experience }(\mathrm{N}=79)\end{array}$} & \multirow[t]{2}{*}{$\begin{array}{c}\text { Previous Additional Jail Experience } \\
\text { Officers' Hierarchy of Motivations For } \\
\text { Joining }\end{array}$} & \multicolumn{2}{|c|}{$\begin{array}{l}\text { Previous Additional } \\
\text { Jail Experience } \\
(\mathrm{N}=34)\end{array}$} \\
\hline & (49) & $32.0 \%$ & & (18) & $52.9 \%$ \\
\hline “The challenge/ excitement” & $(41)$ & $51.9 \%$ & "The challenge/ excitement" & (18) & $52.9 \%$ \\
\hline "For the money" & $(27)$ & $34.2 \%$ & "The job looked interesting” & (16) & $47.1 \%$ \\
\hline “To help reduce re-offending” & (18) & $22.8 \%$ & “Just needed a job” & $(12)$ & $35.3 \%$ \\
\hline "Just needed a job” & (18) & $22.8 \%$ & "Friends/ relatives were prison officers" & (6) & $17.6 \%$ \\
\hline "Friends/ relatives were prison officers" & (15) & $19.0 \%$ & “To help reduce re-offending” & (6) & $17.6 \%$ \\
\hline "Always wanted to be a prison officer" & (5) & $6.3 \%$ & "Always wanted to be a prison officer" & $(2)$ & $5.9 \%$ \\
\hline “Other” & (5) & $6.3 \%$ & Job security & (2) & $5.9 \%$ \\
\hline Job security & (4) & $5.1 \%$ & Enjoy working with people & (1) & $2.9 \%$ \\
\hline Enjoy working with people & (3) & $3.8 \%$ & "Power" & $(0)$ & $0.0 \%$ \\
\hline “Power” & (1) & $1.3 \%$ & “Other” & $(0)$ & $0.0 \%$ \\
\hline
\end{tabular}

Note. Multiple responses permitted therefore totals do not equal 100\%. Dotted line indicates the division for Expected N, categories above have positive residuals, categories below have negative residuals. 


\section{$\underline{\text { APPENDIX P }}$}

\section{Previous Occupations OF PRISON OFFICERS AT THE TARGET INSTITUTION}




\section{$\underline{\text { Table P.1 }}$}

Previous Occupations of Prison Officers at the Target Institution

\begin{tabular}{lc}
\hline \multicolumn{1}{c}{ Previous Occupation } & $\begin{array}{c}\text { Prison Officers Involved in } \\
\text { Occupation Group Prior to } \\
\text { Becoming a Prison Officer } \\
\text { (N=115) }\end{array}$ \\
\hline Trades person/ skilled worker e.g. technician & $(64) 55.7 \%$ \\
Unskilled worker e.g. labourer & $(50) 43.5 \%$ \\
Semi-skilled worker e.g. truck driver & $(42) 23.5 \%$ \\
Retail/ sales & $(26) 22.6 \%$ \\
Management e.g. above supervisor role & $(26) 22.6 \%$ \\
Clerical/ office & $(22) 19.1 \%$ \\
Military proper e.g. service person & $(21) 18.3 \%$ \\
Security officer & $(16) 13.9 \%$ \\
Own business/ self employed & $(12) 10.4 \%$ \\
Teacher & $(9) 7.8 \%$ \\
Military other e.g. store-man & $(5) \quad 4.3 \%$ \\
Police e.g. police officer; non-commissioned officer & $(5) 4.3 \%$ \\
Corrections/ Justice Department affiliated & $(4) 3.5 \%$ \\
Home executive e.g. home maker; mother & $(3) 2.6 \%$ \\
Professional e.g. lawyer; accountant & $(1) \quad 0.9 \%$ \\
Unemployed for longer than 6 months & $(0) \quad 0 \%$ \\
\hline
\end{tabular}

Note. Multiple responses permitted therefore totals do not equal 100\%.

${ }^{\mathrm{a}}=$ Note that this figure is less than those that indicated within a separate question on the questionnaire that they had prior military experience. It is unclear why this discrepancy occurred, except to suppose that within this question some respondents assumed that they had already indicated this prior occupation and therefore did not repeat it's inclusion. To remain loyal to the data presented, the figure here has not been amended. 


\section{REFERENCE SECTION}

Adams, R. (1992). Prison riots in Britain and the USA. New York: St Martin's Press.

Altman, I. (1975). The environment and social behavior: Privacy, personal space, territory, crowding. Monteray: Brooks/ Cole Publishing Company.

Ambler, C. (1993a). Troops stand by to guard prisons. The Dominion, $3^{\text {rd }}$ July, 14.

Ambler, C. (1993b). Prisons face more trouble as staff step up pressure. The Dominion, $22^{\text {nd }}$ June, 3.

Arney, G.A., Johnson, A.J., \& Gresson, H.B. (1861). Judges of the Supreme Court: Memorandum of the Judges of the Supreme Court, assembled in conference at Auckland, concerning the gaols of the colony. Appendix to the Journals of the House of Representatives of New Zealand, D-No.2A.

Bain, H., \& Daniels, C. (1996). Security guards may escort prisoners. The Dominion, $18^{\text {th }}$ June, 3.

Baines, D. (2006). 'If you could change one thing': Social service workers and restructuring. Australian Social Work, 59 (1), 20-34.

Baker, D. (2007). Strategic change management in public sector organisations. Oxford: Chandos Publishing.

Baldwin, J.D., \& Baldwin, J.I. (1986). Behavior principles in everyday life. Englewood Cliffs, NJ: Prentice-Hall.

Bass, B.M., \& Avolio, B.J. (1993). Transformational leadership and organizational culture. Public Administration Quarterly, 17 (1), 112-121.

Bates, C. (1997). Rioters said to be drunk on liquor brewed in prison. The Dominion, $7^{\text {th }}$ October, 3.

Bates, C., \& Venter, N. (1997). Prisoners held NZ to ransom say police, MP. The Dominion, $21^{\text {st }}$ October, 1.

Becker, H. (1967). Whose side are we on? Social Problems, 14, 239-247.

Bell, C. (1996). Complaints of hostility on picket lines outside jails. The Dominion, $5^{\text {th }}$ September, 3.

Bell, C. (1997). Prison duty draws staff from defence. The Dominion, $23^{\text {rd }}$ January, 6. 
Bennett, K. (1995). Constitutional issues in cross-gender searches and visual observation of nude inmates by opposite-sex officers: A battle between and within the sexes. The Prison Journal, 75 (1), 90-112.

Berger, P.L., \& Berger, B. (1978). Sociology: A biographical approach. Auckland: Penguin Books.

Beyer, J.M., Hannah, D.R., \& Milton, L.P. (2000). Ties that bind: Culture and attachments in organizations. In N.M. Ashkanay, C.P.M. Wilderom, \& M.F. Peterson (Eds.), Handbook of organizational culture and climate (pp. 323-338). London: Sage Publications.

Boyle, J. (1977). A sense of freedom. London: Pan Books.

Braybrook, B., \& Southey, P. (1992). Census of prison inmates 1991. Wellington: Department of Justice Policy and Research Division.

Britton, D.M. (2007). Gendered organizational logic: Policy and practice in men's and women's prisons. In P. Lewis, \& R. Simpson (Eds.), Gendering emotions in organizations (pp. 263-285). New York: Palgrave MacMillan.

Brown, J., Heidensohn, F. (2000). Gender and policing: Comparative perspectives. London: MacMillan Press.

Burke, M. (1994). Homosexuality as deviance: The case of the gay police officer. British Journal of Criminology, 34 (2), 192-203.

Burns, T., \& Stalker, G.M. (1996). The management of innovation. Oxford: Oxford University Press.

Burton, O. (1945). In prison. Wellington: Reed Publications.

Cameron, N. (1973). Cadet training and the prison service. British Journal of Criminology, 13 (4), 354-372.

CANZ (2005). About CANZ and its officers. Retrieved March 5 ${ }^{\text {th }}, 2005$ from http://www.canz.gen.nz/canz.html

Carlone, D., \& Larson, G.S. (2006). Locating possibilities for control and resistance in a self-help program. Western Journal of Communication, 70 (4), 270-291.

Carlopio, J., Andrewartha, G., \& Armstrong, H. (2005). Developing management skills: A comprehensive guide for leaders. French Forest, NSW: Pearson Education Australia Limited.

Carroll, L. (1974). Hacks, blacks and cons: Race relations in a maximum security prison. Lexington: Lexington Books.

Cavadino, M., \& Dignan, J. (1993). The penal system. London: Sage Publications. 
Census (1875). Results of a census of the colony of New Zealand taken for the night of the $1^{\text {st }}$ March, 1874. Wellington: Government Printer.

Census (1880). Results of a census of the colony of New Zealand taken for the night of the $1^{\text {st }}$ March, 1878. Wellington: Government Printer.

Census (1882). Results of a census of the colony of New Zealand taken for the night of the $3^{\text {rd }}$ April, 1881. Wellington: Government Printer.

Census (1886). Results of a census of the colony of New Zealand taken of the night of the $28^{\text {th }}$ March, 1886. Wellington: Government Printer.

Census (1891). Results of a census of the colony of New Zealand taken of the night of the $5^{\text {th }}$ April, 1891. Wellington: Government Printer.

Census (1897). Results of a census of the colony of New Zealand taken for the night of the $12^{\text {th }}$ April, 1896. Wellington: Government Printer.

Census (1907). Results of a census of the colony of New Zealand taken for the night of the $29^{\text {th }}$ April, 1906. Wellington: Government Printer.

Census (1911). Results of a census of the colony of New Zealand taken for the night of the $2^{\text {nd }}$ April, 1911. Wellington: Government Printer.

Census (1918). Results of a census of the dominion of New Zealand taken for the night of the $15^{\text {th }}$ October, 1916. Part ix: Occupations and unemployment. Wellington: Government Printer.

Census (1946). Dominion of New Zealand. Population census, 1936. Volume 10: Industries and occupations. Wellington: Government Printer.

Census (1948). New Zealand. Population census, 1945. Volume 9: Industries and occupations. Wellington: Census and Statistics Department.

Census (1954). New Zealand. Population census, 1951. Volume 9: Industries, occupations and incomes. Wellington: Government Printer.

Census (1965). New Zealand population census, 1961. Volume 4: Industries and occupations. Wellington: Department of Statistics.

Census (1969). New Zealand census of population and dwellings, 1966. Volume 4: Industries and occupations. Wellington: Department of Statistics.

Census (1974). New Zealand census of population and dwellings, 1971. Volume 4: Industries and occupations. Wellington: Department of Statistics.

Census (1980). New Zealand census of populations and dwellings, 1976. Volume 4: Labour force. Wellington: Department of Statistics. 
Census (1988). 1986 New Zealand census of population and dwellings. Labour force: Part 1, series c, report 4. Wellington: Department of Statistics.

Census (n.d.). New Zealand census of population and dwellings, 1981. Volume 4: Labour force. Wellington: Department of Statistics.

Chan, J.B.L. (1997). Changing police culture: Policing in a multicultural society. Melbourne: Cambridge University Press.

Chan, J.B. (2003). Fair cop: Learning the art of policing. Toronto: University of Toronto Press.

Cheek, F.E., \& Miller, M. (1983). The experience of stress for correction officers: A double-bind theory of correctional stress. Journal of Criminal Justice, 11 (2), 105112.

Clemmer, D. (1940). The prison community. New York: Holt, Rinehart and Winston.

Cloward, R. (1960). Social control within the prison. In R. Cloward (Ed.), Theoretical studies in the social organization of the prison (pp. 20-48). New York: Social Science Research Council.

Cohen, S., \& Taylor, L. (1972). Psychological survival: The experience of long-term imprisonment. Harmondsworth: Penguin Books.

Commission of Inquiry (1965a). Report of the Commission of Inquiry into disturbance at Auckland Prison on 20-21 July, 1965. Wellington: Department of Prisons.

Commission of Inquiry (1965b). Report of the Commission of Inquiry into disturbance at Christchurch Prison 25 July, 1965. Wellington: Department of Prisons.

Conover, T. (2000). New jack. New York: Random House.

Crawley, E. (2004). Doing prison work: The public and private lives of prison officers. Devon: Willan Publishing.

Cressey, D. (1959). Contradictory objectives in complex organizations: The case of the prison. Administrative Science Quarterly, 4 (1), 1-19.

Crouch, B. (1985). Pandora's box: Women guards in men's prisons. Journal of Criminal Justice, 13, 535-548.

Cullen, F.T., Link, B.G., Cullen, J.B., \& Wolfe, N.T. (1990). How satisfying is prison work? A comparative approach. Journal of Offender Counseling Services and Rehabilitation, 14, 89-108.

Currie, C. (1989). Art in prison: An evaluation of a New Zealand prison programme. Wellington: Institute of Criminology Victoria University of Wellington. Dalton, M. (1959). Men who manage. New York: John Wiley. 
Dandridge, T.C., Mitroff, I., \& Joyce, W.F. (1980). Organizational symbolism: A topic to expand organizational analysis. Academy of Management Review, 5, 77-82.

Dane, F.C. (1990). Research methods. Pacific Grove: Brooks/ Cole Publishing Company.

Deal, T.E., \& Kennedy, A.A. (1982). Corporate cultures: The rites and rituals of corporate life. Harmondsworth: Penguin Books.

Department of Corrections (1996a). EEO plan underlines Department's approach. Corrections News, November/ December, 12.

Department of Corrections (1996b). Escape report endorses prison practices. Corrections News, September/ October, 3.

Department of Corrections (1997a). Staff encouraged to apply for unit manager positions. Inside, June, 3.

Department of Corrections (1997b). Working on career qualifications. Corrections News, September, 4.

Department of Corrections (1997c). Competencies recognised by qualifications. Inside, February, 1.

Department of Corrections (1997d). The prison officer development project. Inside, May, 1.

Department of Corrections (1997e). Expectations explained in code. Corrections News, February/ March, 2.

Department of Corrections (1997f). Code of conduct. Wellington: Department of Corrections.

Department of Corrections (1997g). New regional managers. Inside, June, 1.

Department of Corrections (1997h). Integrated offender management system being introduced. Inside, May, 3.

Department of Corrections (1997i). EEO plan ready for distribution. Corrections News, September, 1.

Department of Corrections (1997j). Cultural development. Corrections News, September, 4.

Department of Corrections (1997k). Inspectors improve access with new 0800 number. Corrections News, November, 3.

Department of Corrections (1997l). [Target prison's name] Prison. Departmental History Sheet. Wellington: Department of Corrections. 
Department of Corrections (1998a). The role of the Ombudsmen. Corrections News, March, 10.

Department of Corrections (1998b). Blueprint to achieve EEO goals. Corrections News, August, 6.

Department of Corrections (1998c). Prisoner escort and court custodial services tenders. Corrections News, March, 4.

Department of Corrections (1998d). Prisoner escort provider ready to swing into action. Corrections News, July, 7.

Department of Corrections (1999a). McCarthy’s column. Inside, June, 2.

Department of Corrections (1999b). IOM sentence management shapes up. Inside, October, 6.

Department of Corrections (1999c). The national study of psychiatric morbidity in New Zealand prisons: An investigation of the prevalence of psychiatric disorders among New Zealand inmates. Wellington: Department of Corrections and Ministries of Health and Justice.

Department of Corrections (2000a). Huge participation rate in prisons' skills audit. Corrections News, April, 11.

Department of Corrections (2000b). Prison incident - who're you going to call? Corrections News, August, 1.

Department of Corrections (2001a). First level 4 certificate presented. Inside, October, 1.

Department of Corrections (2001b). Annual report 1 July 2000 - 30 June 2001. Wellington: Department of Corrections.

Department of Corrections (2002a). The way we were - looking back on our prisons. Corrections News, October, 6-7.

Department of Corrections (2002b). IOM - hard facts from all the hard work. Corrections News, March, 6.

Department of Corrections (2003). Facts. Retrieved February 20 2003 from http://www.corrections.govt.nz/facts/key.html

Department of Corrections (2004a). Inmate security classification. Corrections News, September, 10.

Department of Corrections (2004b). Department of Corrections annual report: 1 July 2003 - 30 June 2004. Retrieved January $28^{\text {th }}$, 2005 from http://www.corrections.govt.nz/public/pdf/annualreports/annual-report-2004.pdf 
Department of Corrections (2004c). Treating violent offenders at [Target prison's name] Prison. Corrections News, August, 6-7.

Department of Corrections (2004d). A new dawn for intervention. Corrections News, July, 6-7.

Department of Corrections (2005a). A career in crime: An introduction to careers in the New Zealand Public Prisons Service. Wellington: Department of Corrections.

Department of Corrections (2005b). Public Prisons Service staff and services. Retrieved January $13^{\text {th }}$, 2005 from http://www.corrections.govt.nz/public/aboutus/servicegroupprofiles/publicprisonsserv ice/staffservices/html

Department of Corrections (n.d.). Policy and procedures manual (PPM). Wellington: Department of Corrections.

Department of Justice (1914). Report on Department of Justice, Prisons Branch for the year ended $31^{\text {st }}$ December 1913. Appendix to the Journals of the House of Representatives of New Zealand, H-20.

Department of Justice (1954). A penal policy for New Zealand. Wellington: Department of Justice.

Department of Justice (1955). Report on Department of Justice for the year 1954-55. Appendix to the Journals of the House of Representatives of New Zealand, H-20.

Department of Justice (1988a). Prisons in change: The submission of the Department of Justice to the Ministerial Inquiry into the prison system. Wellington: Department of Justice.

Department Of Justice (1988b). Prisons in change: The submission of the Department of Justice to the Ministerial Inquiry into the prison system. Appendices. Wellington: Department of Justice.

Department of Justice (1990). Major prison reform: Questions and answers on new policy direction. He ara hou. Department of Justice: Wellington.

Department of Justice (1995). Annual report for year ending 30 June 1995. Wellington: Department of Justice.

Dixon, D. (1997). Ethics, law and criminological research. The Australian and New Zealand Journal of Criminology, 30, 211-216.

Dilulio, J.J. (1987). Governing prisons: A comparative study of correctional management. New York: The Free Press.

Douglas, J.D. (1970). Introduction. In J.D. Douglas (Ed.), Observations of deviance (pp. 3-11). New York: Random Houses. 
Douglas, J.D. (1972). Research on deviance. New York: Random House.

Dwan, S. (2004). Changing organisational culture. New Zealand Business, June, 36.

Eldridge, J.E.T. (1971). Max Weber: The interpretation of social reality. London: Michael Joseph Limited.

Farnworth, L. (2007). Women doing a man's job: Female prison officers working in a male prison. In P. Lewis, \& R. Simpson (Eds.), Gendering emotions in organizations (pp. 287-305). New York: Palgrave MacMillan.

Fineman, S., Sims, D., \& Gabriel, Y. (2005). Organizing and organizations. London: Sage Publications.

Fitzgerald, M., \& Sim, J. (1982). British prisons. Oxford: Basil Blackwell.

Fletcher, J. (1978). Situation ethics. In W.T. Reich (Ed.), Encyclopedia of bioethics (pp. 421-424). New York: The Free Press.

Fry, L.J., \& Glaser, D. (1987). Gender differences in work adjustment of prison employees. Journal of Offender Counselling Services and Rehabilitation, 12 (1), 3952.

Foucault, M. (1991). Discipline and punish: The birth of the prison. London: Penguin Books.

Fox, L. (1952). The English prison and borstal systems: An account of the prison and borstal systems in England and Wales after the Criminal Justice Act 1948. London: Routledge and Kegan Paul.

Gabriel, Y. (1994). Organizational nostalgia - reflections on 'the golden age'. In S. Fineman (Ed.), Emotion in organizations (pp. 118-141). London: Sage Publications.

Gagliardi, P. (1990). Symbols and artifacts: Views of the corporate landscape. New York: Walter de Gruyter.

Gagliardi, P. (1996). Exploring the aesthetic side of organizational life. In S.R. Clegg, C. Hardy, \& W.R. Nord (Eds.), Handbook of organizational studies (pp. 565-580). Thousand Oaks, CA: Sage Publications.

Galpin, T.J. (1996). The human side of change: A practical guide to organization redesign. San Francisco: Jossey-Bass Publishers.

Gaols Committee (1878). Report on gaols management, together with minutes of evidence. Appendix to the Journals of the House of Representatives of New Zealand, I-4.

Garabedian, P. (1963). Social roles and the process of socialization in the prison community. Social Problems, 11 (2), 140-153. 
Gardiner, J. (1994). Private jail likely within two years. The Dominion, $1^{\text {st }}$ December, 11.

Genders, E., \& Player, E. (1989). Race relations in prisons. Oxford: Oxford University Press.

Genders, E., \& Player, E. (1995). Grendon: A study of a therapeutic prison. Oxford: Clarendon Press.

Gendreau, P., \& Simpson, G. (1986). Ministerial review of Psychological Services. Wellington: Government Printers.

Giallombardo, R. (1966). Society of women: A study of a women's prison. New York: John Wiley and Sons.

Giddens, A. (1991). Sociology. Cambridge: Polity Press.

Gilbert, N. (1994). Researching social life. London: Sage Publications.

Goff, P. (1994). Private-sector prisons not the answer. The Dominion, $2^{\text {nd }}$ June, 8.

Goffman, E. (1959). The presentation of self in everyday life. New York: Anchor Books.

Goffman, E. (1961). Asylums: Essays on the social situation of mental patients and other inmates. London: Penguin Books.

Goffman, E. (1968). Stigma: Notes on the management of spoiled identity. Harmondsworth: Penguin Books.

Goffman, E. (1974). Frame analysis: An essay on the organization of experience. Cambridge: Harvard University Press.

Hafford-Letchfield, T. (2006). Cultural revolution. Retrieved April 24 $4^{\text {th }}$, 2008 from http://web.ebscohost.com/ehost/detail?vid=5\&hid=17\&sid=fc83877d-d797-4838-92b

Hampton, B. (1993). Prisons and women. Kensington, NSW: New South Wales University Press.

Harris, R. (1977). People in cages: The solution that failed. Auckland: Crimanon.

Harrison, R., \& Stokes, H. (1992). Diagnosing organizational culture. San Diego: Pfeiffer.

Härtel, C.E.J. (2004). Towards a multicultural world: Identifying work systems, practices and employee attitudes that embrace diversity. Australian Journal of Management, 29 (2), 189-200.

Hatch, M.J. (1993). The dynamics of organizational culture. Academy of Management Review, 18, 657-693. 
Hatch, M.J. (1997). Organizational theory and theorizing: Modern, symbolicinterpretive and postmodern perspectives. Oxford: Oxford University Press.

Hatch, M.J. (2000). The cultural dynamics of organizing and change. In N.M. Ashkanay, C.P.M. Wilderom, \& M.F. Peterson (Eds.), Handbook of organizational culture and climate (pp. 245-260). London: Sage Publications.

Hawthorne Experiments (2008). Hawthorne Experiments. Retrieved May 19 ${ }^{\text {th }}, 2008$ from http://en.wikipedia.org/wiki/Hawthorne_experiments

Hay, F., \& Matthews, C.R. (1913). Report on Department of Justice, Prisons Branch for the year ending $31^{\text {st }}$ December 1912. Appendix to the Journals of the House of Representatives of New Zealand, H-20, 1-7.

Heidensohn, F. (1992). Women in control? The role of women in law enforcement. Oxford: Clarendon Press.

Helms Mills, J.C., \& Mills, A.J. (2000). Rules, sensemaking, formative contexts, and discourse in the gendering of organizational culture. In N.M. Ashkanay, C.P.M. Wilderom, \& M.F. Peterson (Eds.), Handbook of organizational culture and climate (pp. 55-70). London: Sage Publications.

Herselman, S. (2004). Cultural diversity, group formation and alienation as constraints on employee interaction in a wholesale company. Anthropology Southern Africa, 27 (3\&4), 122-128.

Hewitt, J.D., Poole, E., \& Regoli, R.M. (1984). Self-reported and observed rule breaking in prison: A look at disciplinary response. Justice Quarterly, 3, 437-448.

Holdaway, S., \& O’Neill, M. (2006). Ethnicity and culture: Thinking about 'police ethnicity'. The British Journal of Sociology, 57 (3), 483-502.

Huggins, M.K., \& Glebbeek, M. (2003). Women studying violent male institutions: Cross-gendered dynamics in police research on secrecy and danger. Theoretical Criminology, 7 (3), 363-387.

Hume, A. (1884). Report on Inspector of Prisons. Appendix to the Journals of the House of Representatives of New Zealand, H-5.

Hume, A. (1889). Report on Department of Justice, Prisons Branch for the year ending $31^{\text {st }}$ December 1888. Appendix to the Journals of the House of Representatives of New Zealand, H-7.

Humphreys, L. (1970). Tearoom trade. Chicago: Aldine.

Innes, M. (2003). Investigating murder. Oxford: Oxford University Press.

Inside Television New Zealand (2007). Kiwis make their own programmes. Retrieved March 6 ${ }^{\text {th }}, 2007$ from http://www.tvnz.co.nz/view/page/826505/823804 
Ironside, A.W. (1910). Gaolers' reports for 1909: Lyttelton Prison. Appendix to the Journals of the House of Representatives of New Zealand, H-20, 8-9.

Ironside, A.W. (1912). Gaolers’ reports for 1911: Lyttelton Prison. Appendix to the Journals of the House of Representatives of New Zealand, H-20, 12-13.

Irwin, J., \& Cressey, D. (1962). Thieves, convicts and the inmate culture. Social Problems, 10 (2), 45-155.

Jacobs, J. (1974). Participant observation in prison. Urban Life and Culture, 3 (2), 221-240.

Jacobs, J.B. (1978). What prison guards think: A profile of the Illinois force. Crime and Delinquency, 24 (1), 185-196.

Jacobs, J. B. (1983). New perspectives on prisons and imprisonment. Ithaca: Cornell University Press.

Jacobs, J.B., \& Kraft, L. (1978). Integrating the keeper: A comparison of black and white guards in Illinois. Social Problems, 25 (3), 304-318.

Jacobs, J.B., \& Retsky, H.G. (1975). Prison guard. Urban Life, 4 (1), 5-29.

James, B., \& Saville-Smith, K. (1990). Gender culture and power: Critical issues in New Zealand society. Auckland: Oxford University Press.

Johnson, R., \& Toch, H. (1982). The pains of imprisonment. London: Sage Publications.

Jones, H., \& Cornes, P. (1977). Open prisons. London: Routledge and Kegan Paul.

Jurik, N.C. (1985a). An officer and a lady: Organizational barriers to women working as correctional officers in men’s prisons. Social Problems, 32 (4), 375-388.

Jurik, N.C. (1985b). Individual and organizational determinants of correctional officer attitudes toward inmates. Criminology, 23 (3), 523-539.

Jurik, N.C. (1988). Striking a balance: Female correctional officers, gender role stereotypes, and male prisons. Sociological Inquiry, 58 (3), 291-305.

Jurik, N.C., \& Halemba, G.J. (1984). Gender, working conditions and the job satisfaction of women in a non-traditional occupation: Female correctional officers in men’s prisons. The Sociological Quarterly, 25, 551-566.

Kakabadse, A. (1982). Culture of the social services. Aldershot, UK: Gower Publishing Company.

Kalimo, R. (1980). Stress in work: Conceptual analysis and a study on prison personnel. Scandinavian Journal of World Environment and Health, 6 (3), 1-149. 
Kanter, R.M. (1977). Men and women of the corporation. New York: Harper and Row Publishers.

Kauffman, K. (1985). Prison officers and their world. Michigan: University Microfilms International Dissertation Services.

Kauffman, K. (1988). Prison officers and their world. Cambridge: Harvard University Press.

Kay, M. (1993a). Prisoners' advocate welcomes report. The Dominion, $26^{\text {th }}$ August, 11.

Kay, M. (1993b). Prison staff say ban on overtime will cause crisis. The Dominion, $2^{\text {nd }}$ June, 3.

Kay, M. (1993c). Jail staff to vote on buyout of allowance. The Dominion, $29^{\text {th }}$ June, 2.

Kay, M. (1994). Prison officer wins damages over assault investigation. The Dominion, $7^{\text {th }}$ May, 3.

Kelsey, J. (1993). Rolling back the state: Privatisation of power in Aotearoa/ New Zealand. Wellington: Bridget Williams Books.

Kelsey, J. (1997). The New Zealand experiment: A world model for structural adjustment. Auckland: Auckland University Press.

Kilroy, S. (1994). Sniping as law allowing private jails gets through. The Dominion, $30^{\text {th }}$ November, 7.

King, R.D., \& McDermott, K. (1995). The state of our prisons. Oxford: Clarendon Press.

Kitchin, P. (1995). Mangaroa still provides a challenge. The Dominion, $5^{\text {th }}$ June, 10.

Klockars, C.B., \& O’Connor, F. (Eds.). (1979). Deviance and decency: The ethics of research with human subjects. New York: Sage.

Klofas, J.M. (1986). Discretion among correctional officers: The influence of urbanization, age and race. International Journal of Offender Therapy and Comparative Criminology, 30 (2), 111-123.

Kono, T., \& Clegg, S.R. (1998). Transformations of corporate culture: Experiences of Japanese enterprises. New York: Walter de Gruyter.

Launay, G., \& Fielding, P.J. (1989). Stress among prison officers: Some empirical evidence based on self report. The Howard Journal of Criminal Justice, 28 (2), 138147.

Lee, R.M. (1993). Doing research on sensitive topics. London: Sage. 
Lee-Treweek, G., \& Linkogle, S. (Eds.). (2000). Danger in the field: Risk and ethics in social research. London: Routledge.

Leo, J. (1997). A no-fault holocaust. United States News and World Report, 123 (3), 14-15.

Liebling, A. (1999). Doing research in prison: Breaking the silence? Theoretical Criminology, 3 (2), 147-173.

Liebling, A., \& Price, D. (2001). The prison officer. Great Britain: HMP Leyhill.

Loader, I., \& Mulcahy, A. (2003). Policing and the condition of England: Memory, politics, and culture. Oxford: Oxford University Press.

Locke, E. (1978). The gaoler. Palmerston North: Dunmore Press.

Logan, B.M. (1993). Ministerial Inquiry into management practices at Mangaroa Prison. Wellington: Ministry of Justice.

Lombardo, L.X. (1981). Guards imprisoned: Correctional officers at work. New York: Elsevier.

Lupton, B. (2000). Maintaining masculinity: Men who do women's work. British Journal of Management, 11, S33-48.

MacKenzie, J. (1996a). Troops take up prison guard duty. The Dominion, $31^{\text {st }}$ August, 1 .

MacKenzie, J. (1996b). Union leader removed from jail manager job. The Dominion, $19^{\text {th }}$ August, 11.

MacKenzie, J. (1996c). Inmates' claim of brutality 'unfounded'. The Dominion, $12^{\text {th }}$ September, 9.

MacKenzie, J. (1996d). Warders get strike-stress counselling. The Dominion, $14^{\text {th }}$ September, 1.

MacKenzie, J. (1996e). Strike 'not to blame' for death of inmate. The Dominion, $4^{\text {th }}$ September, 1 \& 3.

Marquart, J.W. (1983). Cooptation of the kept: Maintaining control in a Southern penitentiary. Michigan: University Microfilms International Dissertation Services.

Marquart, J.W. (1986). Doing research in prison: The strengths and weaknesses of full participation as a guard. Justice Quarterly, 3 (1), 15-32.

Marquart, J.W., \& Crouch, B.M. (1985). Judicial reform and prisoner control: The impact of Ruiz v. Estelle on a Texas penitentiary. Law and Society Review, 19 (4), 557-586. 
Marquart, J.W., \& Roebuck, J.B. (1986). Prison guards and snitches: Social control in a maximum security institution. In K.C. Haas, \& G.P. Alpert (Eds.), The dilemmas of punishment: Readings in contemporary corrections (pp. 158-176). Prospect Heights, Illinois: Waveland Press.

Marquis, C., \& Lounsbury, M. (2007). Vive la resistance: Competing logics and the consolidation of U.S. community banking. Academy of Management Journal, 50 (4), 799-820.

Martin, C. (2000). Doing research in a prison setting. In V. Jupp, P. Davies, \& P. Francis (Eds.), Doing criminological research (pp. 215-232). London: Sage Publications.

Massey, P. (1995). New Zealand: Market liberalisation in a developed economy. London: MacMillan Press.

Mathiesen, T. (1965). The defences of the weak: A Sociological Study of a Norwegian correctional institution. London: Tavistock Publications.

Matthews, R. (1999). Doing time. London: Macmillan Press.

May, E. (1980). A day on the job - in prison. In B.M. Crouch (Ed.), The keepers: Prison guards and contemporary corrections (pp. 138-149). Springfield Illinois: Charles C Thomas Publisher.

McCleary, R. (1960). Communications patterns as bases of systems of authority. In R. Cloward (Ed.), Theoretical studies in the social organization of the prison (pp. 4977). New York: Social Science Research Council.

McEleney, B.L. (1985). Correctional reform in New York. New York: University Press of America.

Ministerial Committee (1989). Prison review. Te ara hou: The new way. Wellington: Government Printer.

Ministerial Report (1910). A scheme for the reorganization of the prison system of New Zealand. Appendix to the Journals of the House of Representatives of New Zealand, $\mathrm{H}-20 \mathrm{~b}$.

Morris, N., \& Hawkins, G. (1970). The honest politicians guide to crime control. Chicago: University of Chicago Press.

Morris, T., \& Morris, P. (1963). Pentonville: A sociological study of an English prison. London: Routledge and Kegan Paul.

Morrison, M. (1989). Methods in sociology. New York: Longman Incorporated.

Moser, C.A., \& Kalton, G. (1977). Survey methods in social investigation. London: Heinemann Educational Books. 
Myers, R. (1995). The prison as a workplace: Prison officers and their work. Sydney, NSW: University of New South Wales.

Newbold, G. (1989). Punishment and politics: The maximum security prison in New Zealand. Auckland: Oxford University Press.

Newbold, G., \& Eskridge, C. (1994). Penal innovation in New Zealand: He ara hou. Journal of Offender Rehabilitation, 20 (3/4), 21-35.

New Zealand Official Yearbook (1895). New Zealand official yearbook 1895. Wellington: Government Printer.

New Zealand Official Yearbook (1902). New Zealand official yearbook 1902, $11^{\text {th }}$ issue. Wellington: Government Printer.

New Zealand Official Yearbook (1903). New Zealand official yearbook 1903, $12^{\text {th }}$ issue. Wellington: Government Printer.

New Zealand Official Yearbook (1913). New Zealand official yearbook 1913, $22^{\text {nd }}$ issue. Wellington: Government Printer.

New Zealand Official Yearbook (1922). New Zealand official yearbook 1921-22, $31^{\text {st }}$ issue. Wellington: Government Printer.

New Zealand Official Yearbook (1926). New Zealand official yearbook 1926, $34^{\text {th }}$ issue. Wellington: Government Printer.

New Zealand Official Yearbook (1930). New Zealand official yearbook 1930, $38^{\text {th }}$ issue. Wellington: Government Printer.

New Zealand Official Yearbook (1937). New Zealand official yearbook 1937, 45 issue. Wellington: Government Printer.

New Zealand Official Yearbook (1943). New Zealand official yearbook 1943, $51^{\text {st }}$ issue. Wellington: Government Printer.

New Zealand Official Yearbook (1948). New Zealand official yearbook 1948, 55 issue. Wellington: Government Printer.

New Zealand Official Yearbook (1953). New Zealand official yearbook 1953, $58^{\text {th }}$ issue. Wellington: Government Printer.

New Zealand Official Yearbook (1957). New Zealand official yearbook 1957, 62 ${ }^{\text {nd }}$ issue. Wellington: Government Printer.

New Zealand Official Yearbook (1962). New Zealand official yearbook 1962, 67 $7^{\text {th }}$ issue. Wellington: Government Printer.

New Zealand Official Yearbook (1967). New Zealand official yearbook 1967, $72^{\text {nd }}$ issue. Wellington: Government Printer. 
New Zealand Official Yearbook (1972). New Zealand official yearbook 1972, $77^{\text {th }}$ issue. Wellington: Government Printer.

New Zealand Official Yearbook (1977). New Zealand official yearbook 1977, $82^{\text {nd }}$ issue. Wellington: Government Printer.

New Zealand Official Yearbook (1982). New Zealand official yearbook 1982, 87 $7^{\text {th }}$ issue. Wellington: Government Printer.

New Zealand Official Yearbook (1988). New Zealand official yearbook 1987-88, 92 ${ }^{\text {nd }}$ issue. Wellington: Government Printer.

New Zealand Official Yearbook (1992). New Zealand official yearbook 1992, 95 ${ }^{\text {th }}$ issue. Wellington: Government Printer.

New Zealand Official Yearbook (1997). New Zealand official yearbook 1997, 100 ${ }^{\text {th }}$ issue. Wellington: Government Printer.

New Zealand Official Yearbook (2002). New Zealand official yearbook 2002, $102^{\text {nd }}$ issue. Auckland: David Bateman Limited.

New Zealand Official Yearbook (2004). New Zealand official yearbook 2004, $104^{\text {th }}$ issue. Auckland: David Bateman Limited.

New Zealand Parliamentary Debates (1945), vol. 272, 312-313.

New Zealand Penal Inspectorate (1990). Invercargill Prison Inquiry. Wellington: Penal Division.

New Zealand Police (1998). Report of the New Zealand Police: For the year ended 30 June 1997. Wellington: New Zealand Police.

NZCTU (2005). Aotearoa New Zealand Council of Trade Unions. Retrieved March $5^{\text {th }}, 2005$ from http://www.nzctu.org.nz/

Oakley, A. (1981). Interviewing women: A contradiction in terms. In H. Roberts (Ed.), Doing feminist research (pp. 30-61). London: Routledge \& Kegan Paul.

Oakley, A. (2000). Experiments in knowing: Gender and method in the social sciences. Cambridge: Polity Press.

O’Connor, M.E. (1976). Prison research: A methodological commentary on being a known observer. Australian and New Zealand Journal of Criminology, 9, 227-234.

Overholser, J.C. (1987). Ethical issues in prison research: A risk/ benefit analysis. Behavioral Sciences and the Law, 5 (2), 187-202.

Owen, B.A. (1985). Race and gender relations among prison workers. Crime and Delinquency, 31 (1), 147-159. 
Owen, B.A. (1988). The reproduction of social control: A study of prison workers at San Quentin. New York: Praeger.

Parenti, C. (1999). Lockdown America: Police and prisons in the age of crisis. London: Verso.

Parisi, N. (Ed.). (1982). Coping with imprisonment. London: Sage Publications.

Parry, K.W., \& Proctor-Thomson, S.B. (2003). Leadership, culture and performance: The case of the New Zealand public sector. Journal of Change Management, 2 (4), 376-399.

Payne, B. (1991). Staunch: Inside the gangs. Auckland: Reed Books.

Pedersen, J.S., \& Sorensen, J.S. (1989). Organisational cultures in theory and practice. Sydney, NSW: Avebury.

Penal Policy Review Committee (1981). Report of the Penal Policy Review Committee. Wellington: Government Printer.

Peterson, C. (1982). Doing time with the boys: An analysis of women correctional officers in all-male facilities. In B. Price, \& N. Sokoloff (Eds.), The criminal justice system and women: Women offenders, victims, workers (pp. 437-460). New York: Clark Boardman.

Philliber, S. (1987). Thy brother's keeper: A review of the literature on correctional officers. Justice Quarterly, 4 (1), 9-37.

Piacentini, L. (2004). Surviving Russian prisons: Punishment, economy and politics in transition. Devon: Willan Publishing.

Pogrebin, M. (1980). Challenge to authority for correctional officers: A conflicting organizational dilemma. Journal of Offender Counselling Services and Rehabilitation, 4 (4), 337-342.

Pratt, J. (1992). Punishment in a perfect society: The New Zealand penal system 18401939. Wellington: Victoria University Press.

Raea, S. (1993). Full inquiry into jail complaints. The Dominion, $25^{\text {th }}$ June, 2.

Rafaeli, A., \& Pratt, M.G. (1993). Tailored meanings: On the meaning and impact of organizational dress. Academy of Management Review, 18, 32-55.

Rafaeli, A., \& Worline, M. (2000). Symbols in organizational culture. In N.M. Ashkanay, C.P.M. Wilderom, \& M.F. Peterson (Eds.), Handbook of organizational culture and climate (pp. 71-84). London: Sage Publications.

Rankin, K. (2006a). Unemployment from 1951. Retrieved September $3^{\text {rd }}$, 2006 from http://keithrankin.co.nz/chart/UnemploymentPostWar.gif 
Rankin, K. (2006b). Declining incomes and economic growth from the 1970s. Retrieved $\quad$ September $\quad 3^{\text {rd }}$, 2006 from http://keithrankin.co.nz/chart/1999714HeraldIncomeGrowth.gif

Remmington, P.W. (1981). Policing: The occupation and the introduction of female officers. Washington: University Press of America.

Reuss-Ianni, E., \& Ianni, F.A.J. (1983). Street cops and management cops: The two cultures of policing. In M. Punch (Ed.), Control in the police organization (pp. 251274). Cambridge, MA: MIT Press.

Roache, M.S., Shouksmith, G.A., Voges, K.E., \& Long, N.R. (1982). The perception of stress by prison officers and their wives: An occupational study. Palmerston North: Massey University.

Robertson, I. (1989). Society: A brief introduction. New York: Worth Publishers Incorporated.

Runciman, W.G. (1978). Max Weber: Selections in translation. Cambridge: Cambridge University Press.

Ryan, P.M. (2001). The Reed dictionary of modern Maori. Auckland: Reed Books.

Sarr, P.A. (1995). Corrections: Recent attempts at workplace reform and managing change in New Zealand prisons. Wellington: Victoria University of Wellington.

Schaefer, R.T. (1989). Sociology: International edition. New York: McGraw-Hill Incorporated.

Schein, E.H. (1985). Organizational culture and leadership: A dynamic view. San Francisco: Jossey-Bass.

Schein, E.H. (1990). Organizational culture. American Psychologist, 45, 109-119.

Schwartzman, H.B. (1993). Ethnography in organizations. London: Sage Publications.

Scraton, P., Sim, J., \& Skidmore, P. (1988). Through the barricades: Prisoner protest and penal policy in Scotland. Journal of Law and Society, 15 (3), 247-262.

Shaw, R. (1999). Rehabilitating the public sector: Alternatives to the Wellington model. In S. Chatterjee, P. Dalziel, C. Eichbaum, P. Harris, B. Philpott, \& R. Shaw (Eds.), The new politics: A third way for New Zealand (pp. 187-218). Palmerston North: Dunmore Press.

Simpson, R. (2007). Emotional labour and identity work of men in caring roles. In P. Lewis, \& R. Simpson (Eds.), Gendering emotions in organizations (pp. 57-74). New York: Palgrave MacMillan. 
Soeters, J.L. (2000). Culture in uniformed organizations. In N.M. Ashkanay, C.P.M. Wilderom, \& M.F. Peterson (Eds.), Handbook of organizational culture and climate (pp. 465-481). London: Sage Publications.

Sopow, E. (2007). The impact of culture and climate on change. Strategic Human Resources Review, 6 (2), 20-23.

Soutar, G.N., \& Williams, T.A. (1985). Prison officers' work attitudes: The influence of background and work experience. Australian and New Zealand Journal of Criminology, 18, 17-24.

Sparks, R., Bottoms, A.E., \& Hay, W. (1996). Prisons and the problem of order. Oxford: Clarendon Press.

Statistics New Zealand (1999). Demographic trends 1998. Wellington: Statistics New Zealand.

Statistics New Zealand (2005). Labour market statistics 2004. Wellington: Statistics New Zealand.

Stotland, E. (1976). Self-esteem and violence by guards and state troopers at Attica. Criminal Justice and Behavior, 3 (1), 85-96.

Stutchbury, G. (1997). Rioting inmates set fires, assault guard. The Dominion, $6^{\text {th }}$ October, 1.

Sykes, G. (1958). The society of captives: A study of a maximum security prison. Princeton: Princeton University Press.

Sykes, G., \& Messinger, S. (1960). The inmate social system. In R. Cloward (Ed.), Theoretical studies in the social organization of the prison (pp. 5-19). New York: Social Science Research Council.

Tauroa, H., \& Tauroa, P. (1994). Te marae: A guide to customs and protocol. Auckland: Reed Books.

Thomas, J.E. (1972). The English prison officer since 1850: A study in conflict. London: Routledge and Kegan Paul.

Tyrrell, M.W.D. (2000). Hunting and gathering in the early silicon age. In N.M. Ashkanay, C.P.M. Wilderom, \& M.F. Peterson (Eds.), Handbook of organizational culture and climate (pp. 85-99). London: Sage Publications.

Unauthored (1993a). Another Mangaroa inquiry to begin. The Dominion, $23^{r d}$ December, 3.

Unauthored (1993b). Suspended guards reinstated. The Dominion, $17^{\text {th }}$ September, 3.

Unauthored (1993c). Prison deal branded a sham. The Dominion, $8^{\text {th }}$ July, 10. 
Unauthored (1993d). Six consortiums to tender for prisons. The Dominion, 23 ${ }^{\text {rd }}$ July, 2 .

Unauthored (1994a). Private prison back on agenda. The Dominion, $4^{\text {th }}$ March, 1.

Unauthored (1994b). Punishment by contract. The Dominion, $13^{\text {th }}$ June, 10.

Unauthored (1994c). Prison pay talks. The Dominion, $9^{\text {th }}$ December, 3.

Unauthored (1994d). Investigation into warders' walkoff. The Dominion, $12^{\text {th }}$ October, 2.

Unauthored (1994e). Warders fight eviction. The Dominion, $25^{\text {th }}$ October, 1.

Unauthored (1995). Tenders called for first private prison. The Dominion, $16^{\text {th }}$ August, 3.

Unauthored (1996a). Jails may use police, army if strike called. The Dominion, $16^{\text {th }}$ August, 3.

Unauthored (1996b). Jail strikers said to be having second thoughts. The Dominion, $9^{\text {th }}$ September, 3 .

Unauthored (1996c). Guards stabbed as inmates escape. The Dominion, $3^{\text {rd }}$ December, 1.

Unauthored (1997a). Officers freed after 26-hour hostage drama. The Dominion, $20^{\text {th }}$ October, 1.

Unauthored (1997b). Inmate's hospital stay sparks inquiry. The Dominion, $22^{\text {nd }}$ September, 3.

Unauthored (1997c). Use of casual staff criticised. The Dominion, $23^{\text {rd }}$ September, 10.

Unauthored (1997d). Prison changes sought after standoff. The Dominion, $7^{\text {th }}$ March, 3.

Unauthored (1997e). Inquiry started after jail fight. The Dominion, $8^{\text {th }}$ March, 7.

Unauthored (1997f). Gelignite at prison. The Dominion, $22^{\text {nd }}$ September, 1.

Unauthored (1997g). Paparua prison guards strike deal. The Dominion, $22^{\text {nd }}$ October, 2.

Unauthored (1997h). Prison officer stabbed repeatedly. The Dominion, 25 ${ }^{\text {th }}$ September, 1.

Unauthored (1997i). Prison guard in hospital after alleged assault. The Dominion, $30^{\text {th }}$ October, 3.

Unauthored (1997j). Officer attacked. The Dominion, $8^{\text {th }}$ November, 1. 
Unauthored (1997k). Call for action after fourth prison assault. The Dominion, $10^{\text {th }}$ November, 3.

Unauthored (1997l). Inmate has 10 years to nibble chocolate bar. The Dominion, $21^{\text {st }}$ June, 8.

van den Berg, P.T., \& Wilderom, C.P.M. (2004). Defining, measuring, and comparing organisational cultures. Applied Psychology: An International Review, 53 (4), 570582.

van Groningen, J. (1981). The job expectations of prison officers: A profile of Victorian recruits. Australian and New Zealand Journal of Criminology, 14 (1), $40-$ 48.

Vasil, A. (1993a). Crisis in prisons as dispute escalates. The Dominion, $5^{\text {th }}$ July, 1.

Vasil, A. (1993b). Unlocked: The end of dispute. The Dominion, $9^{\text {th }}$ July, 9.

Vasil, A. (1993c). Jail staff take deal but many voted 'no'. The Dominion, $4^{\text {th }}$ August, 3.

Vasil, A. (1993d). Justice staff given $\$ 3.5 \mathrm{~m}$ bonus. The Dominion, $12^{\text {th }}$ November, 3.

Vasil, A. (1995a). Breakaway jail staff expected to back pay deal. The Dominion, $17^{\text {th }}$ February, 12.

Vasil, A. (1995b). Prison officers win housing court case. The Dominion, $28^{\text {th }}$ January, 1.

Vasil, A. (1996). Department threatening jail officers says union. The Dominion, $2^{\text {nd }}$ September, 3.

Viitanen, E., \& Piirainen, A. (2003). Reflection on work culture: The key to success. Reflective Practice, 4 (2), 179-192.

Wacquant, L. (2002). The curious eclipse of prison ethnography in the age of mass incarceration. Ethnography, 3 (4), 371-397.

Walsh, E.J. (1975). Dirty work, race, and self-esteem. Ann Arbor, MI: Institute of labor and industrial relations.

Walsh, P. (1991). The State Sector Act 1988. In J. Boston, J. Martin, J. Pallot, \& P. Walsh (Eds.), Reshaping the state: New Zealand's bureaucratic revolution (pp. 5280). Auckland: Oxford University Press.

Walsh, P. (1994). Has the evil been remedied: The development of public sector unionism in New Zealand. In P. Walsh (Ed.), Pioneering New Zealand labour history (pp. 112-125). Palmerston North: The Dunmore Press Limited. 
Walters, S. (1993). Changing the guard: Male correctional officers' attitudes toward women as co-workers. Journal of Offender Rehabilitation, 20 (1-2), 47-60.

Weber, M. (1962). Basic concepts in sociology. New York: The Citadel Press.

Webster, I.W., Porritt, D.W., \& Brennan, P. (1983). Reported health, life-style and occupational stress in prison officers. Community Health Studies, 7 (3), 266-277.

Wellman, C. (1978). Relativism. In W.T. Reich (Ed.), Encyclopedia of bioethics (pp. 454-487). New York: The Free Press.

Westmarland, L. (2001). Gender and policing: Sex, power and police culture. Cullompton: Willan Publishing.

Wheeler, S. (1961). Socialization in correctional communities. The American Sociological Review, 26, 697-712.

Whitehead, J.T., \& Lindquist, C.A. (1986). Correctional officer job burnout: A path model. Journal of Research in Crime and Delinquency, 23 (1), 23-42.

Whiteley, A. (1995). Managing change: A core values approach. Melbourne, VIC: MacMillan Education Australia Limited.

Whiteley, A., \& Whiteley, J. (2007). Core values and organizational change: Theory and practice. New Jersey: World Scientific.

Whyte, W.F. (1984). Learning from the field: A guide from experience. Beverly Hills: Sage Publications.

Whyte, W.F. (1993). Street corner society: The social structure of an Italian slum. Chicago: The University of Chicago Press.

Wicks, R. (1980). Guard! Society’s professional prisoner. Houston: Gulf Publishers.

Williams, T.A., \& Soutar, G.N. (1984). Levels of custody and attitude differences among prison officers: A comparative study. Australian and New Zealand Journal of Criminology, 17 (2), 87-94.

Wilson, F.M. (1995). Organizational behaviour and gender. London: McGraw-Hill Book Company.

Wolcott, H. (1995). The art of fieldwork. Walnut Creek: Sage Publications.

Young, M. (1991). An inside job: Policing and police culture in Britain. Oxford: Clarendon Press.

Zamble, E., \& Porporino, F.J. (1988). Coping, behavior, and adaptation in prison inmates. New York: Springer-Verlag. 
Zimmer, L. (1989). Solving women's employment problems in corrections: Shifting the burden to administrators. Women and Criminal Justice, 1 (1), 55-79.

Zimmer, L., \& Jacobs, J.B. (1981). Challenging the Taylor Law: Prison guards on strike. Industrial and Labor Relations Review, 34 (4), 531-544. 